\title{
THE CYCLOTOMIC IWASAWA MAIN CONJECTURE FOR HILBERT CUSPFORMS WITH COMPLEX MULTIPLICATION
}

\author{
TAKASHI HARA AND TADASHI OCHIAI
}

\begin{abstract}
We deduce the cyclotomic Iwasawa main conjecture for Hilbert modular cuspforms with complex multiplication from the multivariable main conjecture for CM number fields. To this end, we study in detail the behaviour of the $p$-adic $L$-functions and the Selmer groups attached to CM number fields under specialisation procedures.
\end{abstract}

\section{CONTEnTs}

1. Introduction

2. The analytic side

2.1. Classical theory on Hilbert modular cuspforms

2.2. The cyclotomic $p$-adic $L$-function for Hilbert modular cuspforms

2.3. Katz, Hida and Tilouine's $p$-adic $L$-functions for CM number fields

2.4. Comparison of the $p$-adic $L$-functions

3. The algebraic side

3.1. Selmer groups

3.2. Exact control theorem

3.3. Greenberg's criterion for almost divisibility

3.4. Inductive specialisation of the characteristic ideals

3.5. Application to the Iwasawa Main Conjecture

Appendix A. Complex multiplication of Hilbert modular cuspforms

Appendix B. Comparison of the global and local duality pairings

References

\section{INTRODUCTION}

The cyclotomic Iwasawa main conjecture for elliptic cuspforms describes the mysterious relation between the Selmer groups (algebraic objects) and the p-adic L-functions (analytic objects) attached to elliptic cuspforms. When the cuspform $f$ under consideration does not have complex multiplication, the cyclotomic Iwasawa main conjecture for $f$ is valid under some technical conditions thanks to Kato04, Theorem 17.4] and [SU14, Theorem 3.6.4]. When the cuspform $f$ under consideration has complex multiplication, one can deduce the validity of the cyclotomic Iwasawa main conjecture for $f$ from the two-variable Iwasawa main conjecture for the imaginary quadratic field $F$ via the cyclotomic specialisation (such

2010 Mathematics Subject Classification. 11R23 (Primary), 11F41, 11F67 (Secondaries).

Key words and phrases. Iwasawa main conjecture, Hilbert modular forms, $p$-adic $L$-functions, Selmer groups, complex multiplication.

This work is partially supported by KAKENHI (Grant-in-Aid for Exploratory Research: Garant Number 24654004, Grant-in-Aid for Scientific Research (B): Grant Number 26287005, and Grant-in-Aid for Young Scientists (B): Grant Number 26800014). 
an observation has been already made when $f$ is a cuspform of weight two associated to a CM elliptic curve at [Ru91], for example). The main purpose of this article is to generalise this procedure and deduce the cyclotomic Iwasawa main conjecture for Hilbert modular cuspforms with complex multiplication from the multivariable Iwasawa main conjecture for CM fields.

Let $p \geq 5$ be a prime number which is fixed throughout the article. We also fix a complex embedding $\iota_{\infty}: \overline{\mathbb{Q}} \hookrightarrow \mathbb{C}$ and a $p$-adic embedding $\iota_{p}: \overline{\mathbb{Q}} \hookrightarrow \overline{\mathbb{Q}}_{p}$ of an algebraic closure $\overline{\mathbb{Q}}$ of the field of rationals $\mathbb{Q}$. Let $F$ be a CM number field of degree $2 d$ and $F^{+}$its maximal totally real subfield. We assume that all prime ideals of $F^{+}$lying above $p$ are unramified over $\mathbb{Q}$ and split completely in the quadratic extension $F / F^{+}$. We denote by $I_{F}$ (resp. $I_{F^{+}}$) the set of all embeddings of $F$ (resp. $F^{+}$) into the fixed algebraic closure $\overline{\mathbb{Q}}$ of the rational number field. We choose and fix a $p$-ordinary CM type $\Sigma$ of $F$, which is a subset of $I_{F}$ satisfying several conditions (see Section 2.3.1 for the definition of $p$-ordinary CM types).

We denote by $\widetilde{F}$ the composition of all $\mathbb{Z}_{p}$-extensions of $F$ (in $\overline{\mathbb{Q}}$ ). It is well known that $\operatorname{Gal}(\widetilde{F} / F)$ is isomorphic to the free $\mathbb{Z}_{p}$-module of rank $d+1+\delta_{F, p}$, where $\delta_{F, p}$ denotes the Leopoldt defect for $F$ and $p$. We abbreviate the composite field of $\widetilde{F}$ and $F\left(\mu_{p}\right)$ as $\widetilde{F}_{\infty}$. For each finite abelian extension $\mathrm{K}$ of $F$ which contains $F\left(\mu_{p}\right)$ and is linearly disjoint from $\widetilde{F}$ over $F$, we define $\widetilde{\mathrm{K}}_{\infty}^{\mathrm{CM}}$ as the composite field $\mathrm{K} \widetilde{F}$.

We consider a größencharacter $\eta$ of type $\left(A_{0}\right)$ on $F$. Assume that $\eta$ is ordinary with respect to the (fixed) p-ordinary CM type $\Sigma$, or in other words, assume that $\eta$ is unramified at the set $\Sigma_{p}$ of places of $F$ corresponding to the $p$-adic embeddings $\iota_{p} \circ \sigma$ for $\sigma$ in $\Sigma$. By virtue of global class field theory, there exists a canonical $p$-adic Galois character $\eta^{\text {gal }}: G_{F} \rightarrow \overline{\mathbb{Q}}_{p}^{\times}$ corresponding to $\eta$ (we shall review the construction of $\eta^{\text {gal }}$ in Section 2.1.1). Then, as we shall recall later in Section 3.1.4, there exists a finite abelian extension $K / F$ and a character $\psi: \operatorname{Gal}(K / F) \rightarrow \overline{\mathbb{Q}}_{p}^{\times}$of finite order such that $\eta^{\text {gal }} \psi^{-1}$ factors through the Galois groups of $\widetilde{F}_{\infty} / F$. Throughout Introduction, we denote by $\mathcal{O}$ the ring of integers of an appropriate finite extension of $\mathbb{Q}_{p}$ which contains the image of $\eta^{\text {gal }}$.

Now let us briefly explain our main results without precision of notation. In Section 2.3 we shall introduce the notion of Katz, Hida and Tilouine's $p$-adic $L$-function $\mathcal{L}_{p, \Sigma}^{\mathrm{KHT}}(F)$ for the CM number field $F$, which is constructed as an element of $\widehat{\mathcal{O}}^{\mathrm{ur}}\left[\left[\mathrm{Gal}\left(F_{\mathfrak{C} p} / F\right)\right]\right]$ (see Theorem 2.19 for details) where $\widehat{\mathcal{O}}^{\text {ur }}$ denotes the composition $\mathcal{O} \widehat{\mathbb{Z}}_{p}^{\text {ur }}$. We denote by $\mathcal{L}_{p}^{\Sigma}(\psi)$ (a certain modification of) the $\psi$-branch of $\mathcal{L}_{p, \Sigma}^{\mathrm{KHT}}(F)$, the image of $\mathcal{L}_{p, \Sigma}^{\mathrm{KHT}}(F)$ under the $\psi$-twisting map $\widehat{\mathcal{O}}^{\mathrm{ur}}\left[\left[\operatorname{Gal}\left(F_{\mathfrak{C} p \infty} / F\right)\right]\right] \rightarrow \widehat{\mathcal{O}}^{\mathrm{ur}}\left[\left[\operatorname{Gal}\left(\widetilde{F}_{\infty} / F\right)\right]\right] ;\left.g \mapsto \psi(g) g\right|_{\widetilde{F}_{\infty}}$. For an arbitrary continuous character $\rho: \operatorname{Gal}\left(\widetilde{F}_{\infty} / F\right) \rightarrow \mathcal{O}^{\times}$, we denote by $\operatorname{Tw}_{\rho}$ the $\rho$-twisting map

$$
\widehat{\mathcal{O}}^{\mathrm{ur}}\left[\left[\operatorname{Gal}\left(\widetilde{F}_{\infty} / F\right)\right]\right] \longrightarrow \widehat{\mathcal{O}}^{\mathrm{ur}}\left[\left[\operatorname{Gal}\left(\widetilde{F}_{\infty} / F\right)\right]\right] ; g \mapsto \rho(g) g .
$$

Under these settings, we state our main results as follows. The first theorem concerns the analytic part of our main results.

Theorem A (=Corollary 2.25). Let $\eta$ be a größencharacter of type $\left(A_{0}\right)$ on $F$ which is ordinary with respect to a p-ordinary $C M$ type $\Sigma$. We choose a finite abelian extension $K$ of $F$ and a character $\psi$ of $\operatorname{Gal}(K / F)$ such that $\eta^{\text {gal }} \psi^{-1}$ factors through the Galois groups of $\widetilde{F}_{\infty} / F$. Then the image of the twisted (multivariable) $p$-adic L-function $\operatorname{Tw}_{\eta^{\text {gal }}} \psi^{-1}\left(\mathcal{L}_{p}^{\Sigma}(\psi)\right)$ under the cyclotomic specialisation map

$$
\widehat{\mathcal{O}}^{\mathrm{ur}}\left[\left[\operatorname{Gal}\left(\widetilde{F}_{\infty} / F\right)\right]\right] \rightarrow \widehat{\mathcal{O}}^{\mathrm{ur}}\left[\left[\operatorname{Gal}\left(F\left(\mu_{p^{\infty}}\right) / F\right)\right]\right] ;\left.g \mapsto g\right|_{F\left(\mu_{p} \infty\right)}
$$


coincides, up to a (componentwise) nonzero constant multiple, with the cyclotomic p-adic $L$-function $\mathcal{L}_{p}^{\text {cyc }}(\vartheta(\eta)) \in \mathcal{O}^{\text {ur }}\left[\left[\operatorname{Gal}\left(F\left(\mu_{p} \infty\right) / F\right)\right]\right] \otimes_{\mathbb{Z}_{p}} \mathbb{Q}_{p}$ associated to the Hilbert modular form $\vartheta(\eta)$ obtained as the theta lift of $\eta$. Furthermore if the conjecture on the ratio of complex periods (see Conjecture 2.26 for details) is true, they coincide up to a (componentwise) p-adic unit multiple.

The algebraic parts of our main results consist of two ingredients: the exact control theorem for the Selmer groups (Theorem $\mathrm{B}$ ) and the triviality of pseudonull submodules for the Pontrjagin duals of the (strict) Selmer groups (Theorem C). We denote by $\mathcal{A}_{\eta}^{\mathrm{CM}}$ (resp. by $\left.\mathcal{A}_{\eta}^{\text {cyc }}\right)$ the cofree module of corank one over $\mathcal{O}\left[\left[\mathrm{Gal}\left(\widetilde{F}_{\infty} / F\right)\right]\right]$ (resp. over $\left.\mathcal{O}\left[\left[\operatorname{Gal}\left(F\left(\mu_{p^{\infty}}\right) / F\right)\right]\right]\right)$ on which an element $g$ of the absolute Galois group $G_{F}$ acts as the multiplication by $\left.\eta^{\text {gal }}(g) g\right|_{\widetilde{F}_{\infty}}$ (resp. by $\left.\left.\eta^{\text {gal }}(g) g\right|_{F\left(\mu_{p} \infty\right)}\right)$. Let us define $\mathfrak{A}^{\text {cyc }}$ as the kernel of the cyclotomic specialisation map $\mathcal{O}\left[\left[\operatorname{Gal}\left(\widetilde{F}_{\infty} / F\right)\right]\right] \rightarrow \mathcal{O}\left[\left[\operatorname{Gal}\left(F\left(\mu_{p^{\infty}}\right) / F\right)\right]\right] ;\left.g \mapsto g\right|_{F\left(\mu_{p}\right)}$. Then we note that $\mathcal{A}_{\eta}^{\text {cyc }}$ coincides with the maximal $\mathfrak{A}^{\text {cyc }}$-torsion submodule $\mathcal{A}_{\eta}^{\mathrm{CM}}\left[\mathfrak{A}^{\text {cyc }}\right]$ of $\mathcal{A}_{\eta}^{\mathrm{CM}}$. By a general recipe due to Greenberg, we define the Selmer group $\operatorname{Sel}_{\mathcal{A}_{\eta}^{\mathrm{CM}}}^{\Sigma}$ (resp. $\left.\mathrm{Sel}_{\mathcal{A}_{\eta}^{\text {cyc }}}^{\Sigma}\right)$ as a subgroup of the global Galois cohomology $H^{1}\left(F, \mathcal{A}_{\eta}^{\mathrm{CM}}\right)\left(\operatorname{resp} . H^{1}\left(F, \mathcal{A}_{\eta}^{\text {cyc }}\right)\right)$ satisfying local conditions obtained by ordinary filtrations (refer to Definition 3.1). The following exact control theorem describes the behaviour of the Selmer group $\operatorname{Sel}_{\mathcal{A}_{\eta}^{\text {CM }}}^{\Sigma}$ under specialisation procedures:

Theorem B (=Theorem 3.18). Let $\eta, K$ and $\psi$ be as in Theorem A. Assume further that the following condition is fulfilled:

(ntr): for each maximal ideal $\mathfrak{M}$ of the semilocal Iwasawa algebra $\mathcal{O}\left[\left[\operatorname{Gal}\left(\widetilde{F}_{\infty} / F\right)\right]\right]$ and for each prime ideal $\mathcal{P}$ of $F$ lying above $p, \mathcal{A}_{\eta}^{\mathrm{CM}}[\mathfrak{M}]$ is nontrivial as a $D_{\mathcal{P}}$-module.

Then the natural map

$$
\operatorname{Sel}_{\mathcal{A}_{\eta}^{\mathrm{cyc}}}^{\Sigma}=\operatorname{Sel}_{\mathcal{A}_{\eta}^{\mathrm{CM}\left[\mathfrak{A}^{\mathrm{cyc}}\right]}}^{\Sigma} \longrightarrow \operatorname{Sel}_{\mathcal{A}_{\eta}^{\mathrm{CM}}}^{\Sigma}\left[\mathfrak{A}^{\mathrm{cyc}}\right]
$$

induced by the inclusion $\mathcal{A}_{\eta}^{\mathrm{cyc}}=\mathcal{A}_{\eta}^{\mathrm{CM}}\left[\mathfrak{A}^{\text {cyc }}\right] \rightarrow \mathcal{A}_{\eta}^{\mathrm{CM}}$ is an isomorphism.

Let $M_{\Sigma_{p}}$ be the maximal abelian pro- $p$ extension of $\widetilde{K}_{\infty}^{\mathrm{CM}}$ unramified outside the places of $\widetilde{K}_{\infty}^{\mathrm{CM}}$ above the set of places $\Sigma_{p}$ of $F$ as introduced previously. We denote the Galois group of $M_{\Sigma_{p}} / \widetilde{K}_{\infty}^{\mathrm{CM}}$ by $X_{\Sigma_{p}}$. Then the module $X_{\Sigma_{p}, \mathcal{O}}=X_{\Sigma_{p}} \otimes_{\mathbb{Z}_{p}} \mathcal{O}$ is naturally regarded as a compact $\mathcal{O}\left[\left[\operatorname{Gal}\left(\widetilde{K}_{\infty}^{\mathrm{CM}} / F\right)\right]\right]$-module. We denote by $X_{\Sigma_{p},(\psi)}$ the $\psi$-isotypic quotient of $X_{\Sigma_{p}, \mathcal{O}}$; namely, it is defined as the scalar extension $X_{\Sigma_{p}, \mathcal{O}} \otimes_{\mathcal{O}[\mathrm{Gal}(K / F)]} \mathcal{O}\left[\mathrm{Gal}\left(F\left(\mu_{p}\right) / F\right)\right]$ of $X_{\Sigma_{p}, \mathcal{O}}$ with respect to the $\psi$-twisting map $\mathcal{O}[\operatorname{Gal}(K / F)] \rightarrow \mathcal{O}\left[\operatorname{Gal}\left(F\left(\mu_{p}\right) / F\right)\right] ;\left.g \mapsto \psi(g) g\right|_{F\left(\mu_{p}\right)}$. Then one observes that the Pontrjagin dual of the Selmer group $\operatorname{Sel}_{\mathcal{A}_{\eta}^{\text {CM }}}^{\Sigma}$ is pseudoisomorphic to $X_{\Sigma_{p},(\psi)} \otimes_{\mathcal{O}} \mathcal{O}\left(\eta^{\text {gal },-1} \psi\right)$ as a module over $\mathcal{O}\left[\left[\operatorname{Gal}\left(\widetilde{F}_{\infty} / F\right)\right]\right]$ (see Proposition 3.16 for details). The next theorem implies that $X_{\Sigma_{p},(\psi)}$ has no nontrivial pseudonull submodules under certain conditions, analogously to results on algebraic structure of Iwasawa modules in classical Iwasawa theory mainly due to Iwasawa and Greenberg [Gr76]:

Theorem C (=Corollary 3.33). Let $\eta, K$ and $\psi$ be as in Theorem $A$, Assume that the cardinality of $\operatorname{Gal}(K / F)$ is relatively prime to $p$ and the character $\psi$ satisfies the nontriviality condition $(\mathrm{ntr})_{\psi}$, which is described analogously to the condition (ntr) in Theorem B (see the statement of Lemma 3.21 for details). Then $X_{\Sigma_{p},(\psi)}$ has no nontrivial pseudonull $\mathcal{O}\left[\left[\operatorname{Gal}\left(\widetilde{F}_{\infty} / F\right)\right]\right]$-submodules. 
Note that $\mathcal{O}\left[\left[\operatorname{Gal}\left(\widetilde{F}_{\infty} / F\right)\right]\right]$ is a semilocal Iwasawa algebra each of whose components is isomorphic to $\mathcal{O}[[\operatorname{Gal}(\widetilde{F} / F)]]$, and the completed group algebra $\mathcal{O}[[\operatorname{Gal}(\widetilde{F} / F)]]$ is isomorphic to the formal power series ring in $\left(d+1+\delta_{F, p}\right)$-variables over $\mathcal{O}$. Thus, the statement above means that $X_{\Sigma_{p},(\psi)}$ has no nontrivial pseudonull submodules over each of such components.

Theorem C shall be used to study the basechange compatibility of the characteristic ideal of $X_{\Sigma_{p},(\psi)} \otimes_{\mathcal{O}} \mathcal{O}\left(\eta^{\text {gal },-1} \psi\right)$ (or equivalently, of the characteristic ideal of the Pontrjagin dual of $\operatorname{Sel}_{\mathcal{A}_{\eta}^{\text {CM }}}^{\Sigma}$ ) under specialisation procedures. We carefully discuss this problem by utilising Theorem $\mathrm{C}$ and certain inductive arguments. For details, see Section 3.4.

Combining these results, we finally obtain the following theorem, which is the main result of this article.

Theorem D (=Theorem 3.41). Let $\eta$ be a größencharacter of type $\left(A_{0}\right)$ on $F$ which is ordinary with respect to a p-ordinary CM type $\Sigma$. Assume that all of the following three conditions are fulfilled

- the größencharacter $\eta$ satisfies the nontriviality condition (ntr) introduced in Theorem B:

- the Iwasawa main conjecture for the CM number field $F$ is true for the branch character $\psi$ chosen as above; that is, the equality

$$
\left(\mathcal{L}_{p}^{\Sigma}(\psi)\right)=\operatorname{Char}_{\mathcal{O}\left[\left[\operatorname{Gal}\left(\widetilde{F}_{\infty} / F\right)\right]\right]}\left(X_{\Sigma_{p},(\psi)}\right)
$$

holds as an equation of ideals of $\widehat{\mathcal{O}}^{\mathrm{ur}}\left[\left[\operatorname{Gal}\left(\widetilde{F}_{\infty} / F\right)\right]\right]$ (see Definition 3.22 for the definition of the characteristic ideal $\operatorname{Char}_{\mathcal{O}\left[\left[\operatorname{Gal}\left(\widetilde{F}_{\infty} / F\right)\right]\right]}\left(X_{\Sigma_{p},(\psi)}\right)$ over the semilocal Iwasawa algebra $\left.\mathcal{O}\left[\left[\operatorname{Gal}\left(\widetilde{F}_{\infty} / F\right)\right]\right]\right)$;

- in each component of the semilocal Iwasawa algebra $\widehat{\mathcal{O}}^{\mathrm{ur}}\left[\left[\mathrm{Gal}\left(\widetilde{F}_{\infty} / F\right)\right]\right]$, the cyclotomic p-adic L-function $\mathcal{L}_{p}^{\mathrm{cyc}}(\vartheta(\eta))$ of $\vartheta(\eta)$ does not vanish.

Then the cyclotomic Iwasawa main conjecture for the p-stabilised Hilbert eigencuspform $f_{\eta}=\vartheta(\eta)^{p-s t}$ with complex multiplication is true up to $\mu$-invariants; in other words, we have the equality of ideals of $\widehat{\mathcal{O}}^{\text {ur }}\left[\left[\operatorname{Gal}\left(F\left(\mu_{p}\right) / F\right)\right]\right] \otimes_{\mathbb{Z}_{p}} \mathbb{Q}_{p}$

$$
\left(\mathcal{L}_{p}^{\text {cyc }}(\vartheta(\eta))\right)=\operatorname{Char}_{\mathcal{O}\left[\left[\operatorname{Gal}\left(F\left(\mu_{p} \infty\right) / F\right)\right]\right]}\left(\operatorname{Sel}_{\mathcal{A}_{\vartheta(\eta)}^{\text {cyc }}}^{\Sigma, \vee}\right),
$$

where the superscript $\vee$ denotes the Pontrjagin dual. If the conjecture on the ratio of complex periods (Conjecture 2.26) is true, the equality above holds in $\mathcal{O}\left[\left[\operatorname{Gal}\left(F\left(\mu_{p^{\infty}}\right) / F\right)\right]\right]$.

Recently Fabio Mainardi and Ming-Lun Hsieh have thoroughly studied the Iwasawa main conjecture for CM number fields, and Hsieh's results combined with Leopoldt conjecture imply its validity for $\widetilde{F}_{\infty} / F$ under certain technical assumptions (see Remark 3.42 for details). Hence Theorem $\mathrm{D}$ combined with Hsieh's results and Leopoldt conjecture guarantees the existence of Hilbert modular cuspforms with complex multiplication for which the cyclotomic Iwasawa main conjecture is true.

The detailed content of this article is as follows. After a brief review on basic facts of größencharacters of type $\left(A_{0}\right)$ and (adèlic) Hilbert modular cuspforms, we shall introduce in Section 2 two $p$-adic $L$-functions of different type: the cyclotomic $p$-adic $L$-functions associated to (nearly p-ordinary) Hilbert modular cuspforms (in Section 2.2) and Katz, Hida and Tilouine's $p$-adic $L$-functions for CM number fields (in Section 2.3). We compare their interpolation formulae in detail in Section 2.4 when the Hilbert modular cuspform under consideration has complex multiplication, and then verify Theorem $\mathrm{A}$ based on this 
comparison. Section 3 is devoted to the algebraic parts of our results. There we introduce various Selmer groups and compare them at a certain extent of precision in Section 3.1. We then prove the Exact Control Theorem (Theorem B) in Section [3.2, and discuss the basechange compatibility of characteristic ideals of the Pontrjagin duals of Selmer groups by recursively applying Greenberg's criterion for almost divisibility in Section 3.4 (we shall briefly review results of Greenberg on the almost divisibility of the Selmer groups in Section [3.3). In this process Theorem $\mathrm{C}$ is proved as the first step of our induction argument (see Section 3.4 .4 for details). Combining all the results obtained in Sections 2 and3, we deduce in Section 3.5 the cyclotomic Iwasawa main conjecture for Hilbert modular cuspforms with complex multiplication (at least up to a nonzero constant multiple) from the (multivariable) Iwasawa main conjecture for CM number fields via the cyclotomic specialisation (Theorem D). In Appendix A, we verify several basic properties of Galois representations associated to Hilbert modular cuspforms with complex multiplication after Ribet's arguments for elliptic cuspforms with complex multiplication employed in Ri77. Appendix $B$ is devoted to the verification of Proposition 3.40 in Section 3 , which is one of the technical keys to our algebraic main results.

Notation. We mainly use the fraktur $\mathfrak{r}$ for the ring of integers of an algebraic number field (which is often regarded as the base field of a certain motive); the calligraphic $\mathcal{O}$ is kept to denote the ring of integers for a p-adic field (which is often regarded as the coefficient field of the $p$-adic realisation of a certain motive). We denote the absolute norm of a fractional ideal $\mathfrak{a}$ of an algebraic number field by $\mathcal{N} \mathfrak{a}$.

Throughout this article $p$ denotes a prime number which is larger than or equal to 5 . We fix an algebraic closure $\overline{\mathbb{Q}}$ of the rational number field $\mathbb{Q}$ and regard all algebraic number fields (that is, all finite extensions of $\mathbb{Q}$ ) as subfields of $\overline{\mathbb{Q}}$. We also fix an embedding $\iota_{\infty}: \overline{\mathbb{Q}} \hookrightarrow \mathbb{C}$ of $\overline{\mathbb{Q}}$ into the complex number field $\mathbb{C}$, and an embedding $\iota_{p}: \overline{\mathbb{Q}} \hookrightarrow \overline{\mathbb{Q}}_{p}$ of $\overline{\mathbb{Q}}$ into a fixed algebraic closure $\overline{\mathbb{Q}}_{p}$ of the $p$-adic number field $\mathbb{Q}_{p}$ respectively.

For an algebraic number field $K$, we denote by $\mathbb{A}_{K}$ (resp. $\mathbb{A}_{K}^{\times}$) the ring of adèles (resp. the group of idèles) of $K$. The finite part (resp. the archimedean part) of the ring of adèles $\mathbb{A}_{\mathrm{K}}$ is denoted by $\mathbb{A}_{\mathrm{K}}^{f}$ (resp. $\left.\mathbb{A}_{\mathrm{K}}^{\infty}\right)$. We associate a modulus $\prod_{v} v^{\operatorname{ord}_{v}(x)}$ to every idèle $x$ in $\mathbb{A}_{\mathrm{K}}^{\times}$, where $v$ runs over all places of $\mathrm{K}$. In this article we only consider moduli associated to finite idèles, and hence we always identify a modulus $\prod_{v} v^{\operatorname{ord}_{v}(x)}$ with the corresponding fractional ideal $\prod_{v} \mathfrak{P}_{v}^{\text {ord }_{v}(x)}$ of $\mathrm{K}$, where $\mathfrak{P}_{v}$ denotes the prime ideal associated to $v$.

We shall fix the notion of the standard additive character throughout this article. For each archimedean place $v$ of an algebraic number field $\mathrm{K}$ and for each $x_{v} \in \mathrm{K}_{v}$, we define the local additive character $\mathbf{e}_{\mathrm{K}_{v}}: \mathrm{K}_{v} \rightarrow \mathbb{C}^{\times}$by

$$
\mathbf{e}_{\mathrm{K}_{v}}\left(x_{v}\right)= \begin{cases}\exp \left(2 \pi \sqrt{-1} x_{v}\right) & \text { if } v \text { is real, } \\ \exp \left(2 \pi \sqrt{-1} x_{v} \bar{x}_{v}\right) & \text { if } v \text { is complex }\end{cases}
$$

where $\bar{x}_{v}$ denotes the complex conjugate of $x_{v}$. For each nonarchimedean place $v$ of $\mathrm{K}$, we define $\mathbf{e}_{\mathrm{K}_{v}}$ as

$$
\mathbf{e}_{\mathrm{K}_{v}}\left(x_{v}\right)=\exp \left(-2 \pi \sqrt{-1} \operatorname{Tr}_{\mathrm{K} / \mathbb{Q}}\left(\tilde{x}_{v}\right)\right)
$$

Here $\tilde{x}_{v}$ denotes an arbitrary element of $\bigcup_{n=1}^{\infty} \mathfrak{P}_{v}^{-n}$ (regarded as a $\mathfrak{r}_{\mathrm{K}}$-submodule of $\mathrm{K}$ ) such that $\tilde{x}_{v}-x_{v}$ is contained in the ring of integers of $\mathrm{K}_{\mathfrak{P}_{v}}$. The adèlic standard additive character $\mathbf{e}_{\mathbb{A}_{K}}: \mathbb{A}_{K} / K \rightarrow \mathbb{C}^{\times}$is defined as the product of local standard characters for all places of $\mathrm{K}$. We also define $\mathbf{e}_{\mathbb{A}_{\mathrm{K}}^{\infty}}$ as the product of all archimedean local additive characters. 
Let $\mathbb{C}_{p}$ be the $p$-adic completion of the fixed algebraic closure $\overline{\mathbb{Q}}_{p}$ of $\mathbb{Q}_{p}$ and $\mathcal{O}_{\mathbb{C}_{p}}$ its ring of integers. For the ring of integers $\mathcal{O}$ of a finite extension of $\mathbb{Q}_{p}$, we denote a discrete valuation ring $\mathcal{O} \widehat{\mathbb{Z}}_{p}^{\text {ur }}$ by $\widehat{\mathcal{O}}^{\text {ur }}$ where $\widehat{\mathbb{Z}}_{p}^{\text {ur }}$ is the $p$-adic completion of the maximal unramified extension of $\mathbb{Z}_{p}$.

In this article we adopt the geometric normalisation of global class field theory; more precisely, let $\mathrm{L} / \mathrm{K}$ be a finite abelian extension of algebraic number fields. Then the reciprocity map $(-, \mathrm{L} / \mathrm{K}): \mathbb{A}_{\mathrm{K}}^{\times} \rightarrow \mathrm{Gal}(\mathrm{L} / \mathrm{K})$ is normalised to map a uniformiser $\varpi_{\mathfrak{p}}$ of a prime ideal $\mathfrak{p}$ relatively prime to the conductor of the extension $\mathrm{L} / \mathrm{K}$ to the geometric Frobenius element $\operatorname{Frob}_{\mathfrak{p}}$ in $\operatorname{Gal}(\mathrm{L} / \mathrm{K})$; that is, $a^{\left(\varpi_{\mathfrak{p}}, \mathrm{L} / \mathrm{K}\right)^{-1}} \equiv a^{q_{\mathfrak{p}}}(\bmod \mathfrak{p})$ holds for each $a$ in $\mathfrak{r}_{\mathrm{K}}$ where $q_{\mathfrak{p}}$ denotes the cardinality of the residue field $\mathfrak{r}_{\mathrm{K}} / \mathfrak{p}$.

If $\mathrm{K}$ is an algebraic number field, the absolute Galois group $\mathrm{Gal}(\overline{\mathbb{Q}} / \mathrm{K})$ is denoted by $G_{\mathrm{K}}$. For a place $v$ of $\mathrm{K}$, we denote by $D_{v}$ and $I_{v}$ the decomposition group and the inertia group at $v$ respectively. For a (possibly infinite) abelian Galois extension $\mathrm{L} / \mathrm{K}$ of $\mathrm{K}$ and the ring of integer $\mathcal{O}$ of a finite extension of $\mathbb{Q}_{p}$, we define $\mathcal{O}[[\mathrm{Gal}(\mathrm{L} / \mathrm{K})]]^{\sharp}$ as the free $\mathcal{O}[[\mathrm{Gal}(\mathrm{L} / \mathrm{K})]]$ module of rank one on which $G_{\mathrm{K}}$ acts via the universal tautological character

$$
G_{\mathrm{K}} \rightarrow \mathcal{O}[[\mathrm{Gal}(\mathrm{L} / \mathrm{K})]]^{\times} ;\left.g \mapsto g\right|_{\mathrm{L}} .
$$

We finally remark that, as for Hodge-Tate $p$-adic representations, the Hodge-Tate weights are normalised so that the Hodge-Tate weight of the $p$-adic cyclotomic character $\chi_{p \text {,cyc }}$ is -1 .

\section{The ANALYTIC SIDE}

We shall develop in this section the analytic parts of our main results. We first present a brief overview of classical theory on Hilbert modular cuspforms in Section 2.1, and introduce the notion of the p-adic L-functions associated to Hilbert modular cuspforms in Section 2.2. We then introduce another type of $p$-adic $L$-functions in Section 2.3; Katz, Hida and Tilouine's p-adic L-functions for CM number fields. In Section 2.4, we consider the cyclotomic specialisation of (appropriately twisted) Katz, Hida and Tilouine's $p$-adic $L$-function, and compare it with the $p$-adic $L$-function of a Hilbert modular cuspform with complex multiplication.

2.1. Classical theory on Hilbert modular cuspforms. This subsection is devoted to an overview of classical (complex) theory on (adèlic) Hilbert modular cuspforms. After a brief review of größencharacters of type $\left(A_{0}\right)$ in Section 2.1.1, we define Hilbert modular cuspforms of double digit weight after Hida, and summarise basic facts on their Fourier expansions and associated (complex) $L$-functions in Section 2.1.2. Section 2.1.3 is a survey of the theory on Hecke operators for Hilbert modular cuspforms. We then introduce in Section 2.1.4 the notion of Galois representations associated to Hilbert modular cuspforms. We finally present the notion of Hilbert modular cuspforms with complex multiplication in Section 2.1.5, which play central roles in the present article.

2.1.1. Generalities on größencharacters of type $\left(A_{0}\right)$. In this paragraph $\mathrm{K}$ denotes a number field (later we only consider the cases where $\mathrm{K}$ is either a totally real number field $\mathrm{F}^{+}$or a CM number field $F$ ). We denote by $I_{\mathrm{K}}$ the set of all embeddings of $\mathrm{K}$ into $\overline{\mathbb{Q}}$. Let $S_{\mathrm{K}}(\mathbb{R})$ (resp. $S_{\mathrm{K}}(\mathbb{C})$ ) denote the set of real places of K (resp. the set of complex places of K), and let $S_{\mathrm{K}, \infty}$ denote the set of archimedian places of $\mathrm{K}$; that is, $S_{\mathrm{K}, \infty}=S_{\mathrm{K}}(\mathbb{R}) \cup S_{\mathrm{K}}(\mathbb{C})$. Recall that each real place corresponds to a unique element of $I_{\mathrm{K}}$ and each complex place corresponds to a unique pair of elements in $I_{\mathrm{K}}$. For each real place, we denote by $\tau_{v}$ the corresponding element in $I_{\mathrm{K}}$. For each complex place $v$, we specify one of the corresponding pair in $I_{\mathrm{K}}$ as 
$\tau_{v}$ and identify $\mathrm{K}_{v}$ with the complex field $\mathbb{C}$ via the embedding $\iota_{\infty} \circ \tau_{v}: \mathrm{K} \hookrightarrow \mathbb{C}$. Then the composite of the other one, which we denote by $\bar{\tau}_{v}$, with $\iota_{\infty}$ is the complex conjugation of $\iota_{\infty} \circ \tau_{v}$.

An idèle class character $\eta: \mathbb{A}_{\mathrm{K}}^{\times} / \mathrm{K}^{\times} \rightarrow \mathbb{C}^{\times}$is called a größencharacter of type $\left(A_{0}\right)$ (or also called an algebraic Hecke character) of $\mathrm{K}$ if its archimedean part is algebraic; namely, there exists an element $\mu=\sum_{\tau \in I_{K}} \mu_{\tau} \tau$ of $\mathbb{Z}\left[I_{\mathrm{K}}\right]$ such that

$$
\eta\left(x_{\infty}\right)=x_{\infty}^{-\mu}:=\prod_{v \in S_{\mathrm{K}}(\mathbb{R})} x_{v}^{-\mu_{\tau_{v}}} \prod_{v \in S_{\mathrm{K}}(\mathbb{C})} x_{v}^{-\mu_{\tau_{v}}} \bar{x}_{v}^{-\mu_{\bar{\tau}_{v}}}
$$

holds for each element $x_{\infty}=\left(x_{v}\right)_{v \in S_{\mathrm{K}, \infty}}$ in the identity component of $\mathbb{A}_{\mathrm{K}}^{\infty, \times}=\left(\mathrm{K} \otimes_{\mathbb{Q}} \mathbb{R}\right)^{\times}$ (that is, for each element $x_{\infty}=\left(x_{v}\right)_{v \in S_{\mathrm{K}, \infty}}$ such that $\left(x_{v}\right)_{v \in S_{\mathrm{K}}(\mathbb{R})}$ is totally positive). Here $\bar{x}_{v}$ denotes the usual complex conjugate of $x_{v}$ in $\mathbb{C}$ (we identify $\mathrm{K}_{v}$ with $\mathbb{C}$ via the specified identification for each complex place $v$ ). The element $\mu$ as above is called the infinity type of $\eta$. It is widely known that $\eta(x) x_{\infty}^{\mu}$ is an algebraic number for each $x$ in $\mathbb{A}_{\mathrm{K}}$ (here $x_{\infty}$ denotes the archimedean part of $x$ ).

For each prime ideal $\mathfrak{l}$ of $\mathbf{K}$, we define $e(\mathfrak{l})$ as the minimum among nonnegative integers $e$ such that the local component $\eta_{\mathfrak{l}}: \mathrm{K}_{\mathfrak{l}}^{\times} \rightarrow \mathbb{C}^{\times}$of $\eta$ at $\mathfrak{l}$ factors through $\mathrm{K}_{\mathfrak{l}}^{\times} /\left(1+\mathfrak{l}^{e}\right) \rightarrow \mathbb{C}^{\times}$. When $\eta$ is unramified at $\mathfrak{l}$, we define $e(\mathfrak{l})$ as 0 . The integral ideal $\mathfrak{C}(\eta)=\prod_{\mathfrak{l}} \mathfrak{e}^{(\mathfrak{l})}$ is called the conductor of $\eta$. We denote by $\eta^{*}$ the ideal character associated to $\eta$; namely, $\eta^{*}$ is the character defined by

$$
\eta^{*}(\mathfrak{a})=\prod_{\mathfrak{l} \mathfrak{C}(\eta)} \eta_{\mathfrak{l}}\left(\varpi_{\mathfrak{l}}^{\operatorname{ord}_{\mathfrak{l}}(\mathfrak{a})}\right)
$$

for each fractional ideal $\mathfrak{a}$ of $\mathrm{K}$ relatively prime to the conductor $\mathfrak{C}(\eta)$, where $\varpi_{\mathfrak{l}}$ denotes a uniformiser of $\mathrm{K}_{\mathfrak{l}}$. Note that the associated ideal character $\eta^{*}$ does not depend on the choice of uniformisers since $\eta$ is unramified at each $\mathfrak{l} \nmid \mathfrak{C}(\eta)$.

Example 2.1 (norm character). The adèlic norm $|\cdot|_{\mathbb{A}_{K}}$ is regarded as a größencharacter of type $\left(A_{0}\right)$ by virtue of Artin's product formula. It has the infinity type $-\sum_{\tau \in I_{K}} \tau$ and the conductor $\mathfrak{r}_{\mathrm{K}}$. The ideal character associated to $|\cdot|_{\mathbb{A}_{K}}$ is $\mathcal{N}_{\mathrm{K}}^{-1}$, the inverse of the absolute norm defined on $\mathrm{K}$. When $\mathrm{K}$ is totally real, an arbitrary größencharacter of type $\left(A_{0}\right)$ defined on $\mathrm{K}$ is described as $\phi|\cdot|_{\mathbb{A}_{K}}^{n}$ for a certain größencharacter $\phi$ of finite order on $\mathrm{K}$ and a certain integer $n$.

Now we associate to $\eta$ a $p$-adic idèle class character $\hat{\eta}: \mathbb{A}_{\mathrm{K}}^{\times} / \mathrm{K}^{\times} \rightarrow \overline{\mathbb{Q}}_{p}^{\times}$as follows; for each prime ideal $\mathfrak{p}$ of $\mathrm{K}$ lying above $p$, we denote by $I_{\mathrm{K}, \mathfrak{p}}$ the subset of $I_{\mathrm{K}}$ consisting of embeddings $\tau$ such that $\iota_{p} \circ \tau$ induces the place associated to $\mathfrak{p}$. Then we put

$$
x_{p}^{-\mu}=\prod_{\mathfrak{p} \mid p \mathfrak{r}_{\mathrm{K}}} x_{\mathfrak{p}}^{-\sum_{\tau \in I_{\mathrm{K}, \mathfrak{p}}} \mu_{\tau}}, \quad \text { for } x_{p}=\left(x_{\mathfrak{p}}\right)_{\mathfrak{p} \mid p \mathfrak{r}_{\mathrm{K}}} \in \mathbb{A}_{\mathrm{K}}^{p, \times}=\left(\mathrm{K} \otimes \mathbb{Q} \mathbb{Q}_{p}\right)^{\times} .
$$

For each idèle $x$ of $\mathrm{K}$, we define $\hat{\eta}(x)$ as

$$
\hat{\eta}(x)=\iota_{p}\left(\eta(x) x_{\infty}^{\mu}\right) x_{p}^{-\mu}
$$

where $x_{p}$ and $x_{\infty}$ respectively denote the $p$-component and the $\infty$-component of $x$. Obviously $\hat{\eta}$ is trivial on $\mathrm{K}^{\times}$and factors through the finite idèle class group $\mathbb{A}_{\mathrm{K}}^{f, \times} / \mathrm{K}^{\times}$by construction. The $p$-adic idèle class character $\hat{\eta}$ constructed as above is called the $p$-adic avatar of $\eta$, while the (complex) character $\eta$ is called the complex avatar of $\hat{\eta}$. Note that, via global class field theory, the $p$-adic avatar $\hat{\eta}$ corresponds to a unique $p$-adic Galois character $\eta^{\text {gal }}$ defined on $\operatorname{Gal}\left(\mathrm{K}_{\mathfrak{C}(\eta)} / \mathrm{K}\right)$ satisfying $\eta^{\text {gal }}\left(\left(x, \mathrm{~K}_{\mathfrak{C}(\eta)} / \mathrm{K}\right)\right)=\hat{\eta}(x)$ for an arbitrary element $x$ 
in $\mathbb{A}_{\mathrm{K}}^{\times}$where $\mathfrak{C}(\eta)$ denotes the conductor of $\eta$ and $\mathrm{K}_{\mathfrak{C}(\eta)}$ denotes the ray class field modulo $\mathfrak{C}(\eta)$ over K. Refer to Notation in Section 1 for our normalisation of the reciprocity map $\left(-, \mathrm{K}_{\mathfrak{C}(\eta) / \mathrm{K}}\right)$. In particular, we have $\eta^{\text {gal }}\left(\right.$ Frob $\left._{\mathfrak{l}}\right)=\eta\left(\varpi_{\mathfrak{l}}\right)=\hat{\eta}\left(\varpi_{\mathfrak{l}}\right)=\eta^{*}(\mathfrak{l})$ for each prime ideal $\mathfrak{l}$ of $\mathrm{K}$ relatively prime to $\mathfrak{C}(\eta)$, where $\varpi_{\mathfrak{l}}$ denotes a uniformiser of the local field $\mathrm{K}_{\mathfrak{l}}$.

2.1.2. Hilbert modular cuspforms of double-digit weight. Let us recall the definition of (adèlic) Hilbert modular cuspforms. We basically follow Hida's description of adèlic Hilbert modular forms in [Hi88, Hi06], although there might be several different manners to introduce them. In particular, we adopt his double-digit weight convention (refer to Hi06, Section 2.3.2]).

Let $F^{+}$be a totally real number field and $\mathfrak{r}_{F^{+}}$the ring of integers of $F^{+}$. We define an algebraic group $G$ over $\mathbb{Z}$ as the Weil restriction of scalars of the general linear group $G L(2) / \mathfrak{r}_{F^{+}}$over $\mathfrak{r}_{F^{+}}$from $\mathfrak{r}_{F^{+}}$to $\mathbb{Z}$. Let $T_{0}$ be the diagonal torus of $G L(2)_{/ \mathfrak{r}_{F}}$ and $T_{G}$ its Weil restriction of scalars from $\mathfrak{r}_{F^{+}}$to $\mathbb{Z}$. The character group $X\left(T_{G}\right)$ of $T_{G}$ is identified with $\mathbb{Z}\left[I_{F^{+}}\right] \times \mathbb{Z}\left[I_{F^{+}}\right]$; specifically, an element $\kappa=\left(\kappa_{1}, \kappa_{2}\right)$ of $\mathbb{Z}\left[I_{F^{+}}\right] \times \mathbb{Z}\left[I_{F^{+}}\right]$corresponds to a unique algebraic character $T_{G / \overline{\mathbb{Q}}} \rightarrow \mathbb{G}_{m} / \overline{\mathbb{Q}}$ which induces

$$
\left(\begin{array}{cc}
x_{1} & 0 \\
0 & x_{2}
\end{array}\right) \mapsto x_{1}^{\kappa_{1}} x_{2}^{\kappa_{2}}, \quad x_{i}^{\kappa_{i}}=\prod_{\tau \in I_{F^{+}}} \tau\left(x_{i}\right)^{\kappa_{i, \tau}} \quad\left(\in \overline{\mathbb{Q}}^{\times}\right) \quad \text { for } i=1,2
$$

on $T_{G}(\mathbb{Q})=F^{+, \times} \times F^{+, \times}$. Here $\kappa_{i}$ denotes an element of $\mathbb{Z}\left[I_{F^{+}}\right]$defined by $\sum_{\tau \in I_{F^{+}}} \kappa_{i, \tau} \tau$ for each $i=1,2$ which satisfies the following condition:

$$
\kappa_{1, \tau}+\kappa_{2, \tau} \text { is a constant independent of } \tau \text { in } I_{F^{+}} .
$$

We denote by $[\kappa]$ the constant value $\kappa_{1, \tau}+\kappa_{2, \tau}$ when the condition (2.3) is satisfied. Note that the diagonal torus $T_{G}$ contains the center $Z$ of $G$, namely the subgroup consisting of all scalar matrices. Let us define another algebraic torus $T$ as the Weil restriction of scalars of the multiplicative group $\mathbb{G}_{m / \mathfrak{r}_{F+}}$ from $\mathfrak{r}_{F^{+}}$to $\mathbb{Z}$. For an integral ideal $\mathfrak{N}$ of $F^{+}$, we consider the following congruence subgroups of $G(\hat{\mathbb{Z}})$ :

$$
\begin{aligned}
\hat{\Gamma}_{0}(\mathfrak{N}) & :=\left\{\left(\begin{array}{ll}
a & b \\
c & d
\end{array}\right) \in G(\hat{\mathbb{Z}}) \mid c \in \mathfrak{N} \hat{\mathfrak{r}}_{F^{+}}\right\}, \\
\hat{\Gamma}_{1}(\mathfrak{N}) & :=\left\{\left(\begin{array}{ll}
a & b \\
c & d
\end{array}\right) \in G(\hat{\mathbb{Z}}) \mid a-1 \in \mathfrak{N} \hat{\mathfrak{r}}_{F^{+}}, c \in \mathfrak{N}_{\mathfrak{x}_{F^{+}}}\right\} .
\end{aligned}
$$

Let $\varepsilon_{+}: \mathbb{A}_{F^{+}}^{\times} / F^{+, \times} \rightarrow \mathbb{C}^{\times}$denote a größencharacter of type $\left(A_{0}\right)$ on $F^{+}$with infinity type $\kappa_{1}+\kappa_{2}-\mathrm{t}$, where the symbol $\mathrm{t}$ denotes the "trace" element $\sum_{\tau \in I_{F^{+}}} \tau$ in $\mathbb{Z}\left[I_{F^{+}}\right]$. We often identify the adèlic points $Z\left(\mathbb{A}_{\mathbb{Q}}\right)$ of the center $Z$ of $G$ with the idèle group $\mathbb{A}_{F^{+}}^{\times}$of $F^{+}$, and regard $\varepsilon_{+}$as a character on $Z\left(\mathbb{A}_{\mathbb{Q}}\right)$. We denote by $\varepsilon: T(\hat{\mathbb{Z}}) \rightarrow \mathbb{C}^{\times}$the restriction of the finite part of $\varepsilon_{+}$to $T(\hat{\mathbb{Z}})=\hat{\mathfrak{r}}_{F^{+}}^{\times}$, where $\hat{\mathfrak{r}}_{F^{+}}^{\times}$denotes the profinite completion of $\mathfrak{r}_{F^{+}}^{\times}$. Then one easily observes that if the conductor $\mathfrak{C}(\varepsilon)$ of $\varepsilon$ contains $\mathfrak{N}$, the map

$$
\left(\begin{array}{ll}
a & b \\
c & d
\end{array}\right) \mapsto \varepsilon\left(a_{\mathfrak{N}}\right)
$$

defines a continuous character $\hat{\Gamma}_{0}(\mathfrak{N}) \rightarrow \mathbb{C}^{\times}$, for which we use the same symbol $\varepsilon$ by abuse of notation. Here $a_{\mathfrak{N}}$ denotes the projection of $a$ to $F_{\mathfrak{N}}^{+}=\prod_{\mathfrak{r} \mid \mathfrak{N}} F_{\mathfrak{l}}^{+}$. We denote the pair of the characters $\left(\varepsilon, \varepsilon_{+}\right)$by $\underline{\varepsilon}$, which shall play a role of a nebentypus character.

Let $\mathfrak{h} \subset \mathbb{C}$ be the Poincaré upper half plane which consists of all complex numbers whose imaginary parts are positive. Then the identity component $G(\mathbb{R})^{+}$of the $\mathbb{R}$-valued points 
$G(\mathbb{R})=G L_{2}(\mathbb{R})^{I_{F}+}$ of $G$ acts on $\mathfrak{h}^{I_{F}+}$ via the coordinatewise Möbius transformation. We now introduce the automorphy factor of weight $\kappa=\left(\kappa_{1}, \kappa_{2}\right)$ by

$$
J_{\kappa}(g, z)=\operatorname{det}(g)^{\kappa_{1}-\mathrm{t}} j(g, z)^{\kappa_{2}-\kappa_{1}+\mathrm{t}} \quad \text { for } g=\left(\begin{array}{ll}
a & b \\
c & d
\end{array}\right) \in G(\mathbb{R})^{+} \text {and } z=\left(z_{\tau}\right)_{\tau \in I_{F^{+}}} \in \mathfrak{h}^{I_{F^{+}}}
$$

where $j(g, z)$ denotes the vector defined by $\left(c_{\tau} z_{\tau}+d_{\tau}\right)_{\tau \in I_{F^{+}}}$. Here we use the following abbreviations on multi-indices:

$$
\operatorname{det}(g)^{\kappa_{1}-\mathrm{t}}=\prod_{\tau \in I_{F^{+}}} \operatorname{det}\left(g_{\tau}\right)^{\kappa_{1, \tau}-1}, \quad j(g, z)^{\kappa_{2}-\kappa_{1}+\mathrm{t}}=\prod_{\tau \in I_{F^{+}}}\left(c_{\tau} z_{\tau}+d_{\tau}\right)^{\kappa_{2, \tau}-\kappa_{1, \tau}+1} .
$$

Definition 2.2 (Hilbert modular cuspforms). Let $\kappa$ be an element of $\mathbb{Z}\left[I_{F^{+}}\right] \times \mathbb{Z}\left[I_{F^{+}}\right]$ for which the condition (2.3) is satisfied, and let $\underline{\varepsilon}=\left(\varepsilon, \varepsilon_{+}\right)$be as above. A complexvalued function $f: G\left(\mathbb{A}_{\mathbb{Q}}\right) \rightarrow \mathbb{C}$ on the adèlic points $G\left(\mathbb{A}_{\mathbb{Q}}\right)$ of $G$ is called a Hilbert modular cuspform of weight $\kappa$, level $\mathfrak{N}$ and nebentypus $\underline{\varepsilon}$ if it satisfies the following three conditions:

(HC1) (automorphy)

let $C_{\mathbf{i}}$ denote the stabiliser subgroup of the vector $\mathbf{i}=(\sqrt{-1}, \ldots, \sqrt{-1}) \in \mathfrak{h}^{I_{F+}}$, which is by definition a subgroup of $G(\mathbb{R})^{+}$. Then the equality

$$
f(\alpha x u w)=\varepsilon_{+}(w) \varepsilon\left(u_{f}\right) f(x) J_{\kappa}\left(u_{\infty}, \mathbf{i}\right)^{-1}
$$

(HC2) (holomorphy)

holds for each $\alpha$ in $G(\mathbb{Q}), w$ in $Z\left(\mathbb{A}_{\mathbb{Q}}\right)$ and $u=u_{f} u_{\infty}$ in $\hat{\Gamma}_{0}(\mathfrak{N}) C_{\mathbf{i}}$;

for each $z$ in $\mathfrak{h}^{I_{F}}$ let us choose an element $u_{\infty}$ of $G(\mathbb{R})^{+}$satisfying $u_{\infty}(\mathbf{i})=z$. Then the function

$$
f_{g}: \mathfrak{h}^{I_{F^{+}}} \rightarrow \mathbb{C} ; z \mapsto f\left(g u_{\infty}\right) J_{\kappa}\left(u_{\infty}, \mathbf{i}\right)
$$

is holomorphic with respect to $z$ for every $g$ in $G\left(\mathbb{A}_{\mathbb{Q}}^{f}\right)$. Note that $f_{g}$ is well defined independently of the choice of each $u_{\infty}$ by virtue of the automorphy (HC1);

(HC3) (cuspidality)

the integral

$$
\int_{\mathbb{A}_{F^{+}} / F^{+}} f\left(\left(\begin{array}{ll}
1 & u \\
0 & 1
\end{array}\right) x\right) d u
$$

vanishes for every $x$ in $G L_{2}\left(\mathbb{A}_{F^{+}}^{f}\right)$ where $d u$ is an additive Haar measure on $\mathbb{A}_{F^{+}} / F^{+}$. We denote by $S_{\kappa}(\mathfrak{N}, \underline{\varepsilon} ; \mathbb{C})$ the complex vector space spanned by all Hilbert modular cuspforms of weight $\kappa$, level $\mathfrak{N}$ and nebentypus $\underline{\varepsilon}$.

It is well known that the space of Hilbert modular cuspforms $S_{\kappa}(\mathfrak{N}, \underline{\varepsilon} ; \mathbb{C})$ is of finite dimension. We often impose the following constraints on weights of Hilbert modular cuspforms.

Definition 2.3. Let $\kappa=\left(\kappa_{1}, \kappa_{2}\right)$ be an element of $\mathbb{Z}\left[I_{F^{+}}\right] \times \mathbb{Z}\left[I_{F^{+}}\right]$for which the condition (2.3) is satisfied.

(1) The element $\kappa$ is called a cohomological weight if the inequality $\kappa_{1}<\kappa_{2}$ holds.

(2) The element $\kappa$ is called a critical weight if it is cohomological and the inequality $\kappa_{1}<0 \leq \kappa_{2}$ holds.

Here inequalities among elements of $\mathbb{Z}\left[I_{F^{+}}\right]$abbreviate corresponding coefficientwise inequalities. For instance, we use the inequality notation $\kappa_{1}<\kappa_{2}$ to express that the inequality $\kappa_{1, \tau}<\kappa_{2, \tau}$ holds for every $\tau$ in $I_{F^{+}}$. 
From now on we always assume that all double-digit weights considered in this article are cohomological.

Now let $f$ denote a Hecke eigencuspform of cohomological weight $\kappa=\left(\kappa_{1}, \kappa_{2}\right)$, level $\mathfrak{N}$ and nebentypus $\underline{\varepsilon}$ (we shall give a brief review on Hecke theory in the next subsection). To each eigencuspform $f$, Blasius and Rogawski [BlRo94] has attached a motive $M(f)_{/ F^{+}}$ defined over $F^{+}$with coefficients in the Hecke field $\mathbb{Q}_{f}$ associated to $f$, which we will introduce later (see the paragraph after Definition [2.7). The motive $M(f)_{/ F^{+}}$is pure of weight $[\kappa]$. For each $\tau$ in $I_{F^{+}}$, the Hodge type of $M(f)_{/ F^{+}}$with respect to the complex embedding $\iota_{\infty} \circ \tau: F^{+} \hookrightarrow \mathbb{C}$ of the field of definition $F^{+}$is given by $\left\{\left(\kappa_{1, \tau}, \kappa_{2, \tau}\right),\left(\kappa_{2, \tau}, \kappa_{1, \tau}\right)\right\}$. In other words, Hida's double-digit weight convention is adapted to the Hodge type of $M(f)_{/ F^{+}}$, and the weight of the cuspform $f$ is critical if and only if the associated motive $M(f)_{/ F^{+}}$is critical in the sense of Deligne [De79].

Remark 2.4 (on nebentypus characters). In [Hi06, Section 2.3.2] Hida introduces more general notion on nebentypus characters; namely, he considers as a nebentypus character a triple $\left(\varepsilon_{1}, \varepsilon_{2} ; \varepsilon_{+}\right)$consisting of finite characters $\varepsilon_{1}, \varepsilon_{2}$ on $T(\hat{\mathbb{Z}})$ and a größencharacter $\varepsilon_{+}$of type $\left(A_{0}\right)$ on $Z\left(\mathbb{A}_{\mathbb{Q}}\right)$ with certain constraints. The nebentypus introduced here is a special one of Hida's general notion. Indeed, our notion of the nebentypus $\underline{\varepsilon}=\left(\varepsilon, \varepsilon_{+}\right)$corresponds to a triple $\left(\varepsilon, 1_{T(\hat{\mathbb{Z}})} ; \varepsilon_{+}\right)$, which satisfies all the required conditions. The space of Hilbert modular cuspforms $S_{\kappa}(\mathfrak{N}, \underline{\varepsilon} ; \mathbb{C})$ with nebentypus of the form $\underline{\varepsilon}=\left(\varepsilon, \varepsilon_{+}\right)$is indeed contained in the space of Hilbert modular cuspforms $S_{\kappa}\left(\hat{\Gamma}_{1}(\mathfrak{N}) ; \mathbb{C}\right)$ of weight $\kappa$ and level $\hat{\Gamma}_{1}(\mathfrak{N})$, and hence we can apply to them general theory on Hilbert modular forms and Hecke algebras of $\hat{\Gamma}_{1}$-level structure developed in Sh78 and Hi88. In particular the (adèlic) Fourier coefficients depend only on fractional ideals of $F^{+}$under such constraints on nebentypus (as we shall see later in Proposition 2.5), and hence our convention on nebentypus characters seems to be well suited to arithmetic problems concerning the $L$-functions associated to Hilbert modular forms.

We next recall the notion of the Fourier expansions of Hilbert modular forms, and then finish this subsection by introducing the (complex) $L$-functions associated to them.

Proposition 2.5. Let $\mathfrak{d}=\mathfrak{d}_{F^{+}}$denote the absolute different of $F^{+}$and let $F_{+}^{+, \times}$denote the set of all totally positive elements of $F^{+}$. Then each Hilbert modular cuspform $f$ belonging to $S_{\kappa}(\mathfrak{N}, \underline{\varepsilon} ; \mathbb{C})$ has the (adèlic) Fourier expansion of the following form for each $x \in \mathbb{A}_{F^{+}}$ and $y \in \mathbb{A}_{F^{+}}^{\times}$:

$$
f\left(\left(\begin{array}{ll}
y & x \\
0 & 1
\end{array}\right)\right)=|y|_{\mathbb{A}_{F^{+}}} \sum_{\xi \in F_{+}^{+, \times}} C(\xi y \mathfrak{d} ; f)\left(\xi y_{\infty}\right)^{-\kappa_{1}} \mathbf{e}_{\mathbb{A}_{F^{+}}^{\infty}}\left(\sqrt{-1} \xi y_{\infty}\right) \mathbf{e}_{\mathbb{A}_{F^{+}}}(\xi x) .
$$

The correspondence $\mathfrak{a} \mapsto C(\mathfrak{a} ; f)$ defines a complex-valued function on the group of fractional ideals of $F^{+}$, which vanishes unless $\mathfrak{a}$ is integral.

This is [Hi88, Proposition 4.1], the proof of which essentially depends on Shimura's classical computation [Sh78, (2.18)]. Refer also to [Hi06, Proposition 2.26]. We call $C(-; f)$ the Fourier coefficient of $f$. A Hilbert modular cuspform $f$ is said to be normalised if its Fourier coefficient $C\left(\mathfrak{r}_{F^{+}} ; f\right)$ at $\mathfrak{r}_{F^{+}}$equals 1 . For a $\mathbb{Q}$-subalgebra $A$ of $\mathbb{C}$, we denote by $S_{\kappa}(\mathfrak{N}, \underline{\varepsilon} ; A)$ the $A$-subspace of $S_{\kappa}(\mathfrak{N}, \underline{\varepsilon} ; \mathbb{C})$ spanned by cuspforms with all Fourier coefficients contained in $A$. 
Now let us assume that the Hilbert modular cuspform $f$ under consideration is a normalised eigenform. The (complex) L-function associated to $f$ is defined as (the meromorphic continuation of) the Dirichlet series

$$
L(f, s)=\sum_{(0) \neq \mathfrak{a} \subseteq \mathfrak{r}_{F^{+}}} \frac{C(\mathfrak{a} ; f)}{\mathcal{N} \mathfrak{a}^{s}} .
$$

For a größencharacter $\theta: \mathbb{A}_{F^{+}}^{\times} / F^{+, \times} \rightarrow \mathbb{C}^{\times}$of type $\left(A_{0}\right)$, we also define the L-function associated to $f$ twisted by $\theta$ as (the meromorphic continuation of)

$$
L(f, \theta, s)=\sum_{(0) \neq \mathfrak{a} \subseteq \mathfrak{r}_{F^{+}}} \frac{C(\mathfrak{a} ; f) \theta^{*}(\mathfrak{a})}{\mathcal{N} \mathfrak{a}^{s}},
$$

where $\theta^{*}$ is the ideal class character which was associated with $\phi$ in (2.1) and $\theta^{*}(\mathfrak{a})$ is defined to be zero if $\mathfrak{a}$ is not relatively prime to the conductor of $\theta$.

2.1.3. Review on Hecke theory. We shall briefly recall Hecke theory on adèlic Hilbert modular cuspforms after [Hi88, Section 2]. As in the previous paragraph, we consider the space $S_{\kappa}(\mathfrak{N}, \underline{\varepsilon} ; \mathbb{C})$ of Hilbert modular cuspforms of weight $\kappa$, level $\mathfrak{N}$ and nebentypus $\underline{\varepsilon}$. Recall that it is contained in the space $S_{\kappa}\left(\hat{\Gamma}_{1}(\mathfrak{N}) ; \mathbb{C}\right)$ of cuspforms of weight $\mathfrak{N}$ and level $\hat{\Gamma}_{1}(\mathfrak{N})$. Now let us define the following monoids:

$$
\begin{aligned}
& \Delta_{0}(\mathfrak{N})=\left\{\left(\begin{array}{ll}
a & b \\
c & d
\end{array}\right) \in M_{2}\left(\hat{\mathfrak{r}}_{F^{+}}\right) \cap G\left(\mathbb{A}_{\mathbb{Q}}^{f}\right) \mid a_{\mathfrak{N}} \in \mathfrak{r}_{F^{+}, \mathfrak{N}}^{\times}, c \in \mathfrak{N}_{F^{+}}\right\}, \\
& \Delta_{1}(\mathfrak{N})=\left\{\left(\begin{array}{ll}
a & b \\
c & d
\end{array}\right) \in M_{2}\left(\hat{\mathfrak{r}}_{F^{+}}\right) \cap G\left(\mathbb{A}_{\mathbb{Q}}^{f}\right) \mid a-1 \in \mathfrak{N} \hat{\mathfrak{r}}_{F^{+}}, c \in \mathfrak{N} \hat{\mathfrak{r}}_{F^{+}}\right\} .
\end{aligned}
$$

Then $\Delta_{0}(\mathfrak{N})$ contains $\hat{\Gamma}_{0}(\mathfrak{N})$ and $\Delta_{1}(\mathfrak{N})$ contains $\hat{\Gamma}_{1}(\mathfrak{N})$ respectively. We thus consider the action of the double coset algebra $R\left(\hat{\Gamma}_{1}(\mathfrak{N}), \Delta_{1}(\mathfrak{N})\right)$ on $S_{\kappa}\left(\hat{\Gamma}_{1}(\mathfrak{N}) ; \mathbb{C}\right)$. We refer the readers to [Sh94, Section 3] for details on the theory of double coset algebras. The action of $R\left(\hat{\Gamma}_{1}(\mathfrak{N}), \Delta_{1}(\mathfrak{N})\right)$ on $S_{\kappa}\left(\hat{\Gamma}_{1}(\mathfrak{N}) ; \mathbb{C}\right)$ is defined as follows. For a cuspform $f$ in $S_{\kappa}\left(\hat{\Gamma}_{1}(\mathfrak{N}), \mathbb{C}\right)$ and an element $\left[\hat{\Gamma}_{1}(\mathfrak{N}) y \hat{\Gamma}_{1}(\mathfrak{N})\right]$ of $R\left(\hat{\Gamma}_{1}(\mathfrak{N}), \Delta_{1}(\mathfrak{N})\right)$, we set

$$
\left.f\right|_{\left[\hat{\Gamma}_{1}(\mathfrak{N}) y \hat{\Gamma}_{1}(\mathfrak{N})\right]}(g)=\sum_{i=1}^{h} f\left(g y_{i}^{-1}\right)
$$

where $\left\{y_{i}\right\}_{i=1, \ldots, h}$ is a representative of the left coset decomposition of $\hat{\Gamma}_{1}(\mathfrak{N}) y \hat{\Gamma}_{1}(\mathfrak{N})$ :

$$
\hat{\Gamma}_{1}(\mathfrak{N}) y \hat{\Gamma}_{1}(\mathfrak{N})=\bigcup_{i=1}^{h} \hat{\Gamma}_{1}(\mathfrak{N}) y_{i} .
$$

Then one easily observes that the action of $R\left(\hat{\Gamma}_{1}(\mathfrak{N}), \Delta_{1}(\mathfrak{N})\right)$ preserves the space $S_{\kappa}(\mathfrak{N}, \underline{\varepsilon} ; \mathbb{C})$ of Hilbert cuspforms with nebentypus $\underline{\varepsilon}$. To describe the structure of the double coset algebra $R\left(\hat{\Gamma}_{1}(\mathfrak{N}), \Delta_{1}(\mathfrak{N})\right)$, we here introduce typical double coset operators which are often quoted as Hecke operators. Choose a uniformiser $\varpi_{\mathfrak{l}}$ of the local field $F_{\mathfrak{l}}^{+}$for each prime ideal $\mathfrak{l}$ of $F^{+}$, and regard $\left(\begin{array}{cc}1 & 0 \\ 0 & \varpi_{\mathfrak{l}}\end{array}\right)$ as an element of $\Delta_{1}(\mathfrak{N})$ whose local component is the 
identity matrix at every place except for $\mathfrak{l}$. Then we define

$$
\begin{aligned}
& T(\mathfrak{l})=\hat{\Gamma}_{1}(\mathfrak{N})\left(\begin{array}{cc}
1 & 0 \\
0 & \varpi_{\mathfrak{l}}
\end{array}\right) \hat{\Gamma}_{1}(\mathfrak{N}) \quad \text { if } \mathfrak{l} \text { does not divide } \mathfrak{N}, \\
& U(\mathfrak{l})=\hat{\Gamma}_{1}(\mathfrak{N})\left(\begin{array}{cc}
1 & 0 \\
0 & \varpi_{\mathfrak{l}}
\end{array}\right) \hat{\Gamma}_{1}(\mathfrak{N}) \quad \text { if } \mathfrak{l} \text { divides } \mathfrak{N}
\end{aligned}
$$

for each prime ideal of $F^{+}$. They are determined independently of the choice of uniformisers. Next let $\mathfrak{b}$ be an integral ideal of $F^{+}$relatively prime to $\mathfrak{N}$. For such an ideal $\mathfrak{b}$, choose a finite idèle $\mathbf{b} \in \hat{\mathfrak{r}}_{F^{+}} \cap \mathbb{A}_{F^{+}}^{\times}$so that it is congruent to 1 modulo $\mathfrak{N}_{F^{+}}$and its associated modulus coincides with $\mathfrak{b}$. Then we set

$$
T(\mathfrak{b}, \mathfrak{b})=\hat{\Gamma}_{1}(\mathfrak{N})\left(\begin{array}{ll}
\mathrm{b} & 0 \\
0 & \mathrm{~b}
\end{array}\right) \hat{\Gamma}_{1}(\mathfrak{N})
$$

which does not depend on the choice of the auxiliary idèle $b$. We also use the notation $S(\mathfrak{b})$ for $T(\mathfrak{b}, \mathfrak{b})$ as in SSh78. By virtue of the general theory, the double coset algebra $R\left(\hat{\Gamma}_{1}(\mathfrak{N}), \Delta_{1}(\mathfrak{N})\right)$ is commutative and is freely generated as a $\mathbb{Z}$-algebra by $T(\mathfrak{l})$ for prime ideals relatively prime to $\mathfrak{N}, U(\mathfrak{l})$ for prime ideals dividing $\mathfrak{N}$ and $S(\mathfrak{l})$ for prime ideals relatively prime to $\mathfrak{N}$. Moreover we obtain the following formula which one can adopt as the definition of the operator $T\left(\mathfrak{l}^{e}\right)$ for prime ideals relatively prime to $\mathfrak{N}$ :

$$
T(\mathfrak{l}) T\left(\mathfrak{l}^{e}\right)=T\left(\mathfrak{l}^{e+1}\right)+\mathcal{N}(\mathfrak{l}) S(\mathfrak{l}) T\left(\mathfrak{l}^{e-1}\right) \quad \text { for each } e \geq 1 .
$$

It is also known that $U\left(\mathfrak{l}^{e}\right)=U(\mathfrak{l})^{e}$ holds for every prime ideal dividing $\mathfrak{N}$ and an arbitrary natural number $e$.

Remark 2.6. In [Hi06] and many other articles of Hida, the action of $R\left(\hat{\Gamma}_{0}(\mathfrak{N}), \Delta_{0}(\mathfrak{N})\right)$ is defined as in the following manner. We first extend the nebentypus character $\underline{\varepsilon}=\left(\varepsilon, \varepsilon_{+}\right)$ to $\Delta_{0}(\mathfrak{N})$ by just setting

$$
\left(\begin{array}{ll}
a & b \\
c & d
\end{array}\right) \mapsto \varepsilon\left(a_{\mathfrak{N}}\right)
$$

Now let $\iota$ denote the standard positive involution on $(2 \times 2)$-matrices defined by

$$
\left(\begin{array}{ll}
a & b \\
c & d
\end{array}\right) \mapsto\left(\begin{array}{cc}
d & -b \\
-c & a
\end{array}\right)
$$

For each $x$ in $\Delta_{0}(\mathfrak{N})$, we define an action of the double coset $\left[\hat{\Gamma}_{0}(\mathfrak{N}) x \hat{\Gamma}_{0}(\mathfrak{N})\right]$ on a Hilbert modular cuspform $f$ belonging to $S_{\kappa}(\mathfrak{N}, \underline{\varepsilon} ; \mathbb{C})$ by

$$
\left.f\right|_{\left[\hat{\Gamma}_{0}(\mathfrak{N}) x \hat{\Gamma}_{0}(\mathfrak{N})\right]}(g)=\sum_{i=1}^{h} \underline{\varepsilon}\left(x_{i}^{-\iota}\right) f\left(g x_{i}^{\iota}\right)
$$

where $\left\{x_{i}\right\}_{i=1, \ldots, h}$ is a representative set of the left coset decomposition of $\hat{\Gamma}_{0}(\mathfrak{N}) x \hat{\Gamma}_{0}(\mathfrak{N})$. For an element $y$ of $\Delta_{1}(\mathfrak{N})$, the action of the $\hat{\Gamma}_{1}(\mathfrak{N})$-double coset $\left[\hat{\Gamma}_{1}(\mathfrak{N}) y \hat{\Gamma}_{1}(\mathfrak{N})\right]$ is compatible with that of the $\hat{\Gamma}_{0}(\mathfrak{N})$-double coset $\left[\hat{\Gamma}_{0}(\mathfrak{N}) y \hat{\Gamma}_{0}(\mathfrak{N})\right]$. Indeed we obtain

$$
\sum_{i=1}^{h} f\left(g y_{i}^{-1}\right)=\sum_{i=1}^{h} f\left(g\left(\operatorname{det} y_{i}\right)^{-1} y_{i}^{\iota}\right)=\sum_{i=1}^{h} \varepsilon_{+}\left(\operatorname{det} y_{i}\right)^{-1} f\left(g y_{i}^{\iota}\right)
$$

for $\hat{\Gamma}_{1}(\mathfrak{N}) y \hat{\Gamma}_{1}(\mathfrak{N})=\bigcup_{i=1}^{h} \hat{\Gamma}_{1}(\mathfrak{N}) y_{i}$, but then $\varepsilon_{+}\left(y_{i}^{-1}\right)$ equals $\varepsilon\left(y_{i}^{-\iota}\right)$ for every $i$ because $y_{i}$ is an element of $\Delta_{1}(\mathfrak{N})$. 
Definition 2.7 (Eigenforms). A Hilbert modular form $f$ belonging to $S_{\kappa}(\mathfrak{N}, \underline{\varepsilon} ; \mathbb{C})$ is called an eigenform if it is a common eigenvector with respect to all double coset operators (or Hecke operators) belonging to $R\left(\hat{\Gamma}_{1}(\mathfrak{N}), \Delta_{1}(\mathfrak{N})\right)$.

By the well-known formula

$$
C\left(\mathfrak{a} ;\left.f\right|_{T(\mathfrak{n})}\right)=\sum_{\mathfrak{b}|\mathfrak{a}, \mathfrak{b}| \mathfrak{n},(\mathfrak{b}, \mathfrak{N})=1} \mathcal{N}(\mathfrak{b}) C\left(\mathfrak{b}^{-2} \mathfrak{n a} ;\left.f\right|_{S(\mathfrak{b})}\right)
$$

(see [Hi88, Corollary 4.2] for instance), one observes that the Fourier coefficient $C(\mathfrak{l} ; f)$ of an eigencuspform $f$ at a prime ideal $\mathfrak{l}$ is obtained as the multiple of $C\left(\mathfrak{r}_{F^{+}} ; f\right)$ and the eigenvalue with respect to the Hecke operator $T(\mathfrak{l})$ (or $U(\mathfrak{l})$ if $\mathfrak{l}$ divides $\mathfrak{N}$ ). Recall that the eigenvalues of the Hecke operators acting on $S_{\kappa}(\mathfrak{N}, \underline{\varepsilon} ; \mathbb{C})$ are algebraic numbers due to $\left[\mathrm{Sh} 78\right.$, Proposition 2.2]. Therefore an eigencuspform $f$ belongs to $S_{\kappa}(\mathfrak{N}, \underline{\varepsilon} ; \overline{\mathbb{Q}})$ if it is normalised. We denote by $\mathbb{Q}_{f}$ the field which one obtains by adjoining to $\mathbb{Q}$ the eigenvalues of the Hecke operators acting on $f$, which we call the Hecke field associated to $f$.

To introduce the notion of near ordinarity, we recall the notion of normalised Hecke operators after [Hi88, Section 3]. Let $\mathbb{Q}\left(\kappa_{1}\right)$ denote a field which one obtains by adjoining to $\mathbb{Q}$ all the elements of the form $x^{\kappa_{1}}$ for $x$ in $F^{+, \times}$. Then $\mathbb{Q}\left(\kappa_{1}\right)$ is a finite extension of $\mathbb{Q}$. Note that $\kappa_{1}$ naturally induces homomorphisms $F^{+, \times} \rightarrow \mathbb{Q}\left(\kappa_{1}\right)^{\times}$and $\mathbb{A}_{F^{+}}^{\times} \rightarrow \mathbb{A}_{\mathbb{Q}\left(\kappa_{1}\right)}^{\times}$. It is

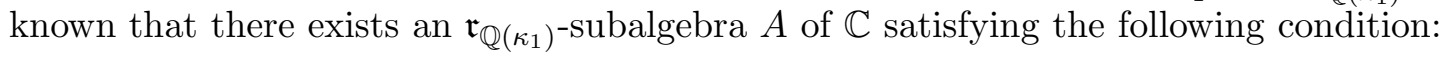

for each element $x$ in $\mathbb{A}_{F^{+}}^{f, \times}$, the modulus associated to $x^{\kappa_{1}}$ is principal as a fractional ideal of $A$.

Refer to [Hi88, p. 310] for details of the existence of such an algebra $A$. Let us choose a uniformiser $\varpi_{\mathfrak{l}}$ of $F_{\mathfrak{l}}^{+}$for each prime ideal $\mathfrak{l}$ of $F^{+}$. We take a generator of the modulus associated to $\varpi_{\mathfrak{l}}^{\kappa_{1}}$ (as a fractional ideal of $A$ ) and denote it by $\left\{\mathfrak{l}^{\kappa_{1}}\right\}$. We also define $\left\{\mathfrak{a}^{\kappa_{1}}\right\}$ for an arbitrary fractional ideal $\mathfrak{a}=\prod_{\mathfrak{l}: \text { prime }} \mathfrak{l}^{(\mathfrak{l})}$ by $\prod_{\mathfrak{l}: \text { prime }}\left\{\mathfrak{k}^{\kappa_{1}}\right\}^{e^{(\mathfrak{l})}}$.

Definition 2.8 (Normalised Hecke operators). We define elements $T_{0}(\mathfrak{l}), U_{0}(\mathfrak{l})$ and $S_{0}(\mathfrak{b})$ of $R\left(\hat{\Gamma}_{1}(\mathfrak{N}), \Delta_{1}(\mathfrak{N})\right) \otimes_{\mathbb{Z}} A$ in the following manner;

$$
\begin{aligned}
T_{0}(\mathfrak{l}) & =\left\{\mathfrak{l}^{\kappa_{1}}\right\}^{-1} T(\mathfrak{l}) \quad \text { for a prime ideal } \mathfrak{l} \text { which does not divide } \mathfrak{N}, \\
U_{0}(\mathfrak{l}) & =\left\{\mathfrak{l}^{\kappa_{1}}\right\}^{-1} U(\mathfrak{l}) \quad \text { for a prime ideal } \mathfrak{l} \text { which divides } \mathfrak{N}, \\
S_{0}(\mathfrak{b}) & =\left\{\mathfrak{b}^{\kappa_{1}}\right\}^{-2} S(\mathfrak{b}) \quad \text { for an integral ideal } \mathfrak{b} \text { which is relatively prime to } \mathfrak{N} .
\end{aligned}
$$

The operators $T_{0}(\mathfrak{l}), U_{0}(\mathfrak{l})$ and $S_{0}(\mathfrak{b})$ are called normalised Hecke operators.

Definition 2.9 (Near ordinarity). Let $\mathfrak{p}$ be a prime ideal of $F^{+}$lying above $p$. A normalised eigencuspform $f$ belonging to $S_{\kappa}(\mathfrak{N}, \underline{\varepsilon} ; \overline{\mathbb{Q}}$ ) is said to be nearly ordinary at $\mathfrak{p}$ (or nearly $\mathfrak{p}$-ordinary) if the eigenvalue of $f$ with respect to the normalised Hecke operator $T_{0}(\mathfrak{p})$ (or $U_{0}(\mathfrak{p})$ if $\mathfrak{p}$ divides the level $\left.\mathfrak{N}\right)$ is a $p$-unit under the specified embedding $\iota_{p}: \overline{\mathbb{Q}} \rightarrow \overline{\mathbb{Q}}_{p}$.

A normalised eigencuspform $f$ which is nearly ordinary at all prime ideals $\mathfrak{p}$ lying above $p$ is said to be nearly ordinary at $p$ (or nearly p-ordinary).

Note that the notion of the normalised Hecke operators does depend on the choice of a generator of $\left\{\mathfrak{p}^{\kappa_{1}}\right\}$, but the notion of the near ordinarity does not depend on it since the $p$-adic valuation of $\left\{\mathfrak{p}^{\kappa_{1}}\right\}$, which is regarded as an element of $\overline{\mathbb{Q}}_{p}$ via the fixed embedding $\iota_{p}$, is well defined independently of the choice of its generator. The normalisation of Hecke operators discussed above is crucial in Hida's theory on $p$-adic Hecke algebras. One of the reasons why it is important is that normalised Hecke operators preserve the space of 
cuspforms with rational or integral coefficients. We omit the details; see [Hi88, Section 4] and [Hi06, Section 2.3.3].

We finally introduce the notion of $p$-stabilisation. Define an operator

$$
V(\mathfrak{b}): S_{\kappa}(\mathfrak{N}, \underline{\varepsilon} ; \overline{\mathbb{Q}}) \rightarrow S_{\kappa}(\mathfrak{b} \mathfrak{N}, \underline{\varepsilon} ; \overline{\mathbb{Q}})
$$

for every integral ideal $\mathfrak{b}$ of $F^{+}$by

$$
\left.f\right|_{V(\mathfrak{b})}(g)=|\mathbf{b}|_{\mathbb{A}_{F^{+}}} f\left(g\left(\begin{array}{cc}
\mathbf{b}^{-1} & 0 \\
0 & 1
\end{array}\right)\right)
$$

where $\mathrm{b}$ is a finite idèle of $F^{+}$whose associated modulus coincides with $\mathfrak{b}$. We readily see that the Fourier coefficient of $\left.f\right|_{V(\mathfrak{b})}$ at $\mathfrak{a}$ is given by $C\left(\mathfrak{b}^{-1} \mathfrak{a} ; f\right)$. Now let $f$ denote a normalised eigencuspform $f$ belonging to $S_{\kappa}(\mathfrak{N}, \underline{\varepsilon} ; \overline{\mathbb{Q}})$ and assume that $f$ is nearly ordinary at $p$. Let $\mathfrak{p}$ be a prime ideal of $F^{+}$lying above $p$ and suppose that the level $\mathfrak{N}$ is not divisible by $\mathfrak{p}$. Note that the eigenvalue of $f$ with respect to the Hecke operator $T_{0}(\mathfrak{p})$ is calculated as $\left\{\mathfrak{p}^{\kappa_{1}}\right\}^{-1} C(\mathfrak{p} ; f)$ which we denote by $C_{0}(\mathfrak{p} ; f)$. The eigenvalue of $f$ with respect to $S_{0}(\mathfrak{p})$ is also calculated as $\left\{\mathfrak{p}^{\kappa_{1}}\right\}^{-2} \varepsilon_{+}^{*}(\mathfrak{p})$. Consider the Hecke polynomial of $f$ with respect to the normalised Hecke operator $T_{0}(\mathfrak{p})$; in other words, consider the quadratic polynomial defined by

$$
1-C_{0}(\mathfrak{p} ; f) X-\mathcal{N} \mathfrak{p}\left\{\mathfrak{p}^{\kappa_{1}}\right\}^{-2} \varepsilon_{+}^{*}(\mathfrak{p}) X^{2}=\left(1-\alpha_{0, \mathfrak{p}} X\right)\left(1-\beta_{0, \mathfrak{p}} X\right) .
$$

We denote two roots of this polynomial (regarded as an elements of $\overline{\mathbb{Q}}$ ) by $\alpha_{0, \mathfrak{p}}$ and $\beta_{0, \mathfrak{p}}$. Since $f$ is nearly ordinarity at $\mathfrak{p}$, the Hecke eigenvalue $C_{0}(\mathfrak{p} ; f)=\alpha_{0, \mathfrak{p}}+\beta_{0, \mathfrak{p}}$ of $f$ with respect to $T_{0}(\mathfrak{p})$ is a $p$-adic unit. This implies that one of the roots has to be a $p$-adic unit (under the fixed embedding $\iota_{p}$ ), which we specify as $\alpha_{0, \mathfrak{p}}$. Let us consider the cuspform $f-\left.\left\{\mathfrak{p}^{\kappa_{1}}\right\} \beta_{0, \mathfrak{p}} f\right|_{V(\mathfrak{p})} \in S_{\kappa}(\mathfrak{p N}, \underline{\varepsilon} ; \overline{\mathbb{Q}})$. Since $\left\{\mathfrak{p}^{\kappa_{1}}\right\} \beta_{0, \mathfrak{p}}$ is a root of the quadratic polynomial $1-C(\mathfrak{p} ; f) X+\mathcal{N} \mathfrak{p} \varepsilon_{+}^{*}(\mathfrak{p}) X^{2}$, the form $f-\left.\left\{\mathfrak{p}^{\kappa_{1}}\right\} \beta_{0, \mathfrak{p}} f\right|_{V(\mathfrak{p})}$ does not depend on the choice of a generator of $\left\{\mathfrak{p}^{\kappa_{1}}\right\}$. It has the same eigenvalues as those of $f$ everywhere except at $\mathfrak{p}$ and has the eigenvalue $\alpha_{0, \mathfrak{p}}$ with respect to $U_{0}(\mathfrak{p})$; hence it is nearly ordinary at $\mathfrak{p}$. Repeating the same procedure for all prime ideals lying above $p$ which do not divide the level $\mathfrak{N}$ of $f$, we obtain the $p$-stabilisation $f^{p \text {-st }}$ of $f$, which is a normalised eigencuspform of level $\mathfrak{N} \prod_{\mathfrak{p} \mid p \mathfrak{r}_{F^{+}}, \mathfrak{p} \mathfrak{n} \mathfrak{i}} \mathfrak{p}$. A normalised nearly $p$-ordinary eigencuspform is called a $p$-stabilised newform if it is obtained as the $p$-stabilisation of a certain primitive form (in the sense of Miyake [Mi71, p. 185]). In particular, the level of a (nearly $p$-ordinary) $p$-stabilised newform is divisible by every prime ideal $\mathfrak{p}$ of $F^{+}$lying above $p$.

2.1.4. Galois representations associated to Hilbert modular cuspforms. We here introduce the notion of Galois representations associated to Hilbert modular cuspforms.

Theorem-Definition 2.10. Let $f$ be a normalised eigencuspform of cohomological weight $\kappa$, level $\mathfrak{N}$ and nebentypus $\underline{\varepsilon}$. Let $\mathcal{K}$ be a finite extension of $\mathbb{Q}_{p}$ containing the Hecke field $\mathbb{Q}_{f}$ of $f$ (under the fixed embedding $\iota_{p}: \overline{\mathbb{Q}} \hookrightarrow \overline{\mathbb{Q}}_{p}$ ). Then there exists a 2 -dimensional Galois representation $V_{f}$ of $G_{F^{+}}$with coefficients in $\mathcal{K}$ such that the equality

$$
\operatorname{det}\left(1-\operatorname{Frob}_{\mathfrak{q}} X ; V_{f}\right)=1-C(\mathfrak{q} ; f) X+\mathcal{N} \mathfrak{q} \varepsilon_{+}^{*}(\mathfrak{q}) X^{2}
$$

holds for every prime ideal $\mathfrak{q}$ which does not divide $p \mathfrak{N}$. Moreover $V_{f}$ is an irreducible representation of $V_{f}$. The Galois representation $V_{f}$ of $G_{F^{+}}$is called the Galois representation associated to $f$. 
The existence of such a Galois representation is established due to results of many people including Ohta [Oh83], Carayol [Ca86], Wiles [Wi88, Taylor [Tay89], and Blasius and Rogawski [BlRo94]. The irreducibility of $V_{f}$ is verified due to Taylor in [Tay95, Theorem 3.1] by the same argument as Ribet's one in [Ri77]. Note that the Galois representation $V_{f}$ is uniquely determined up to scalar multiples by virtue of its irreducibility combined with Čebotarev's density theorem.

When the Hilbert cuspform $f$ is nearly $p$-ordinary, we can also obtain precise information on the local behaviour of the associated representation $V_{f}$ at places above $p$.

Proposition 2.11. Let $f$ be a normalised eigencuspform of cohomological weight $\kappa$, level $\mathfrak{N}$ and nebentypus $\underline{\varepsilon}$ which is nearly ordinary at $p$ and let the other notation be as in TheoremDefinition 2.10. Then, for each place $\mathfrak{p}$ of $F^{+}$lying above $p$, the Galois representation $V_{f}$ associated to $f$ contains a unique 1-dimensional $D_{\mathfrak{p}}$-stable $\mathcal{K}$-subspace $\mathrm{Fil}_{\mathfrak{p}}^{+} V_{f}$ on which the decomposition group $D_{\mathfrak{p}}$ of $G_{F^{+}}$at $\mathfrak{p}$ acts via the $p$-adic character character $\delta_{\mathfrak{p}}: D_{\mathfrak{p}} \rightarrow \mathcal{K}^{\times}$ satisfying

$$
\delta_{\mathfrak{p}}\left(\operatorname{Frob}_{\varpi_{\mathfrak{p}}}\right)=\alpha_{f}\left(\varpi_{\mathfrak{p}}\right)
$$

for every uniformiser $\varpi_{\mathfrak{p}}$ of $F_{\mathfrak{p}}^{+}$. Here Frob $\varpi_{\mathfrak{p}}=\left(\varpi_{\mathfrak{p}}, F_{\mathfrak{p}}^{+, a b} / F_{\mathfrak{p}}^{+}\right)$denotes the Frobenius element corresponding to the uniformiser $\varpi_{\mathfrak{p}}$ via the local reciprocity map. The value $\alpha_{f}\left(\varpi_{\mathfrak{p}}\right)$ appearing in (2.5) is a unique p-adic unit root of the quadratic polynomial

$$
1-\varpi_{\mathfrak{p}}^{-\kappa_{1, \mathfrak{p}}} C(\mathfrak{p} ; f) X-\varpi_{\mathfrak{p}}^{-2 \kappa_{1, \mathfrak{p}}} \mathcal{N} \mathfrak{p} \varepsilon_{+}^{*}(\mathfrak{p}) X^{2}=\left(1-\alpha_{f}\left(\varpi_{\mathfrak{p}}\right) X\right)\left(1-\beta_{f}\left(\varpi_{\mathfrak{p}}\right) X\right)
$$

where $C(\mathfrak{p} ; f)$ denotes the eigenvalue of the Hecke operator $U(\mathfrak{p})$ with respect to $f$, and $\kappa_{1, \mathfrak{p}}=\sum_{\tau:\left(\iota_{p} \circ \tau\right)^{-1}\left(\mathfrak{M}_{\mathbb{Z}_{p}}\right)=\mathfrak{p}} \kappa_{1, \tau}$ denotes the summation of $\kappa_{1, \tau}$ over all $\tau: F^{+} \hookrightarrow \overline{\mathbb{Q}}$ such that $\iota_{p} \circ \tau$ induces the place $\mathfrak{p}$.

Note that the near $p$-ordinarity of $f$ guarantees that the quadratic equation (2.6) indeed has a unique $p$-adic unit root. We also remark that the character $\delta_{\mathfrak{p}}$ is not unramified in general, and hence the equation (2.5) does depend on the choice of a uniformiser $\varpi_{\mathfrak{p}}$ of $F_{\mathfrak{p}}^{+}$, contrary to the cases of $p$-ordinary modular form (of parallel weight).

Proposition 2.11 is first observed by Mazur and Wiles for $p$-ordinary elliptic modular forms of weight 2 [MW84, Chapter 3., Section 2] and then verified by Wiles for $p$-ordinary Hilbert modular forms of parallel weight in WWi86, Theorem 2.2] and [Wi88, Theorem 2]. The general cases have been verified by Hida in [Hi89, Theorem I]. The $D_{\mathfrak{p}}$-stable filtration $\mathrm{Fil}_{\mathfrak{p}}^{+} V_{f} \subset V_{f}$ is used to define the local condition at $\mathfrak{p}$ of Greenberg's Selmer group (see also Section 3.1.1).

2.1.5. Hilbert modular cuspforms with complex multiplication. In this subsection we introduce the notion of Hilbert modular cuspforms with complex multiplication. The following definition is due to Ribet for elliptic modular forms [Ri77, Section 3]:

Definition 2.12 (Cuspform with complex multiplication). Let $\nu: \mathbb{A}_{F^{+}}^{\times} / F^{+, \times} \rightarrow \mathbb{C}^{\times}$be a nontrivial größencharacter of finite order on $F^{+, \times}$. A Hilbert modular eigencuspform $f$ of weight $\kappa$, level $\mathfrak{N}$ and nebentypus $\underline{\varepsilon}$ is said to have complex multiplication by $\nu$ if the equality

$$
C(\mathfrak{l} ; f)=\nu^{*}(\mathfrak{l}) C(\mathfrak{l} ; f)
$$

holds for all prime ideals $\mathfrak{l}$ in a set of prime ideals of density 1 in $\mathfrak{r}_{F^{+}}$.

A Hilbert modular eigencuspform $f$ of weight $\kappa$, level $\mathfrak{N}$ and nebentypus $\underline{\varepsilon}$ is said to have complex multiplication if $f$ has complex multiplication by a certain nontrivial größencharacter $\nu: \mathbb{A}_{F^{+}}^{\times} / F^{+, \times} \rightarrow \mathbb{C}^{\times}$of finite order on $F^{+}$. 
The right hand side of (2.7) is naturally regarded as the Fourier coefficient at $\mathfrak{l}$ of $f \otimes \nu$ : the cuspform $f$ twisted by the größencharacter $\nu$ of finite order (see [Sh78, Proposition 4.5] for the definition of $f \otimes \nu$ ). The nebentypus of $f \otimes \nu$ is easily calculated as $\nu^{2} \varepsilon$, and by comparing eigenvalues of the Hecke operator $S(\mathfrak{l})$ at $f$ and $f \otimes \nu$, we obtain the equality

$$
\varepsilon_{+}^{*}(\mathfrak{l})=\nu^{*}(\mathfrak{l})^{2} \varepsilon_{+}^{*}(\mathfrak{l})
$$

for every prime ideal $\mathfrak{l}$ prime to levels of $f$ and $f \otimes \nu$. Then Čebotarev's density theorem forces $\nu^{2}$ to be trivial, and consequently $\nu$ must be a quadratic character if $f$ has complex multiplication by $\nu$.

An example of Hilbert eigencuspforms which has complex multiplication is obtained as the theta lift of a größencharacter of type $\left(A_{0}\right)$ of a CM number fields. In order to introduce the notion of theta lifts, let us consider a totally imaginary quadratic extension $F$ of $F^{+}$, which is by construction a CM number field. We denote by $c$ a unique nontrivial element of the Galois group $\operatorname{Gal}\left(F / F^{+}\right)$, which is none other than the conplex conjugation. Now let $\Sigma$ denote a CM type of $F$, that is, a subset of $I_{F}$ such that $I_{F}$ is decomposed into the disjoint union of $\Sigma$ and $\Sigma^{c}=\{\sigma \circ c \mid \sigma \in \Sigma\}$. Then we have a canonical bijection $\Sigma \longrightarrow I_{F^{+}}$ via the restriction $\left.\sigma \mapsto \sigma\right|_{F^{+}}$. Since each complex place corresponds to a unique element $\sigma$ of the fixed $p$-ordinary CM-type $\Sigma$, we identify $\Sigma$ with the set of archimedean places of $F$ and, for each $\sigma \in \Sigma$, we specify the identification of $F_{\sigma}$ with $\mathbb{C}$ via the embedding $\iota_{\infty} \circ \sigma: F \hookrightarrow \mathbb{C}$; in other words, we identify $\mathbb{A}_{F}^{\infty}=F \otimes_{\mathbb{Q}} \mathbb{R}$ with $\mathbb{C}^{\Sigma}$ via the isomorphism induced by $x \otimes 1 \mapsto\left(\iota_{\infty} \circ \sigma(x)\right)_{\sigma \in \Sigma}$.

Under these identifications, the infinity type $\mu$ of a größencharacter $\eta: \mathbb{A}_{F}^{\times} / F^{\times} \rightarrow \mathbb{C}^{\times}$ of type $\left(A_{0}\right)$ is described as $\mu=\sum_{\sigma \in \Sigma}\left(\mu_{\sigma} \sigma+\mu_{\bar{\sigma}} \bar{\sigma}\right)$, where $\bar{\sigma}=\sigma \circ c$ denotes a unique element in $\Sigma^{c}$ corresponding to $\sigma \in \Sigma$. A größencharacter $\eta$ of type $\left(A_{0}\right)$ on $F$ is said to be $\Sigma$-admissible (or admissible with respect to $\Sigma$ ) if its infinity type $\mu$ satisfies $\mu_{\sigma}<\mu_{\bar{\sigma}}$ for every $\sigma$ in $\Sigma$. Given an $\Sigma$-admissible infinity type $\mu$ on $F$, we define a cohomological double digit weight $\kappa_{\mu}=\left(\kappa_{\mu, 1}, \kappa_{\mu, 2}\right) \in \mathbb{Z}\left[I_{F^{+}}\right] \times \mathbb{Z}\left[I_{F^{+}}\right]$on $F^{+}$by

$$
\kappa_{\mu}=\left(\left.\sum_{\sigma \in \Sigma} \mu_{\sigma} \sigma\right|_{F^{+}},\left.\sum_{\sigma \in \Sigma} \mu_{\bar{\sigma}} \bar{\sigma}\right|_{F^{+}}\right) .
$$

Given a größencharacter $\eta: \mathbb{A}_{F}^{\times} / F^{\times} \rightarrow \mathbb{C}$ of type $\left(A_{0}\right)$ on $F$, we define $\underline{\varepsilon}_{\eta}=\left(\varepsilon_{\eta}, \varepsilon_{\eta,+}\right)$ by

$$
\underline{\varepsilon}_{\eta}=\left(\left.\nu_{F / F^{+}} \breve{\eta}\right|_{T(\hat{\mathbb{Z}})}, \nu_{F / F^{+}} \breve{\eta}\right)
$$

where $\breve{\eta}$ is defined to be $\left.\eta\right|_{\mathbb{A}_{F^{+}}^{\times}}|\cdot|_{\mathbb{A}_{F^{+}}^{\times}}$and $\nu_{F / F^{+}}$denotes the quadratic character on $\mathbb{A}_{F^{+}}^{\times} / F^{+, \times}$associated to the quadratic extension $F / F^{+}$via global class field theory. Finally let $\mathfrak{D}_{F / F^{+}}$denote the relative discriminant of the quadratic extension $F / F^{+}$.

Proposition-Definition 2.13 (Theta lifts). Let $F$ be a totally quadratic extension of $F^{+}$, $\Sigma$ a CM type of $F$, and $\mathfrak{C}$ an integral ideal of $F$. Let $\eta: \mathbb{A}_{F}^{\times} / F^{\times} \rightarrow \mathbb{C}^{\times}$be a größencharacter of type $\left(A_{0}\right)$ with modulus $\mathfrak{C}$ and suppose that the infinity type $\mu$ of $\eta$ is $\Sigma$-admissible. Then there exists a unique normalised cuspform $\vartheta(\eta)$ of weight $\kappa_{\mu}$, level $\mathfrak{D}_{F / F^{+}} \mathfrak{C C}^{c}$ and nebentypus $\underline{\varepsilon}_{\eta}$ such that its Fourier coefficient $C(\mathfrak{a} ; \vartheta(\eta))$ at an integral ideal $\mathfrak{a}$ of $F^{+}$is given by $\sum_{\mathfrak{A} \subseteq \mathfrak{r}_{F},(\mathfrak{A}, \mathfrak{C})=1 ; \mathfrak{A} \mathfrak{A} c=\mathfrak{a}} \eta^{*}(\mathfrak{A})$. The cuspform $\vartheta(\eta)$ is a common eigenvector of $T(\mathfrak{l})$ for every prime ideal $\mathfrak{l}$ relatively prime to $\mathfrak{D}_{F / F^{+}} \mathfrak{C C}^{c}$. Furthermore if the modulus $\mathfrak{C}$ of $\eta$ coincides with the conductor $\mathfrak{C}(\eta)$ of $\eta$, the resulting cuspform $\vartheta(\eta)$ is primitive in the sense of Miyake [Mi71, p. 185]. 
The normalised Hilbert modular cuspform $\vartheta(\eta)$ defined as above is called the theta lift of the größencharacter $\eta$ of $\left(A_{0}\right)$ on $F$. See [Ge75, Section 7B] for the proof of the proposition, which is based upon Hecke theory on GL(2) through representation theoretic arguments.

By its explicit description, the Fourier coefficient $C(\mathfrak{l} ; \vartheta(\eta))$ of $\vartheta(\eta)$ at a prime ideal $\mathfrak{l}$ equals 0 if $\mathfrak{l}$ is inert in $F$; in other words, $C(\mathfrak{l} ; \vartheta(\eta))$ vanishes when $\nu_{F / F^{+}}^{*}(\mathfrak{l})$ equals 0 or -1 . We thus readily observe that $\vartheta(\eta)$ has complex multiplication by $\nu_{F / F^{+}}$. Conversely, if a Hilbert cuspform $f$ belonging to $S_{\kappa}(\mathfrak{N}, \underline{\varepsilon} ; \overline{\mathbb{Q}})$ has complex multiplication by a quadratic character $\nu$, there exists a totally imaginary quadratic extension $F$ of $F^{+}$such that $\nu$ is the character associated to $F / F^{+}$and $f$ is described as a linear combination of theta lifts of appropriate größencharacters of type $\left(A_{0}\right)$ on $F$. We strongly believe that this fact is fairly well known, but we shall give a proof of this fact with the language of Galois representation at Proposition A.1 in Appendix A.

In order to let the theta lift $\vartheta(\eta)$ be nearly p-ordinary, we must impose the following ordinarity condition on the totally imaginary quadratic extension $F / F^{+}$:

- $\left(\operatorname{ord}_{F / F^{+}}\right)$all places of $F^{+}$lying above $p$ split completely in $F$.

Then due to the ordinarity condition $\left(\operatorname{ord}_{F / F^{+}}\right.$), there exists a p-ordinary CM type $\Sigma$ of $F$; that is, $\Sigma$ is a CM type such that two embeddings $\iota_{p} \circ \sigma$ and $\iota_{p} \circ \sigma \circ c$ of $F$ into $\overline{\mathbb{Q}}_{p}$ define different places of $F$ (lying above $p$ ) for each $\sigma$ in $\Sigma$. See Section 2.3.1 for details on p-ordinary CM types.

Now, under the ordinarity condition $\left(\operatorname{ord}_{F / F^{+}}\right)$, let $\Sigma$ be a $p$-ordinary CM type of $F$ and $\eta: \mathbb{A}_{F}^{\times} / F^{\times} \rightarrow \mathbb{C}^{\times}$a $\Sigma$-admissible größencharacter of type $\left(A_{0}\right)$ on $F$. We say $\eta$ to be ordinary with respect to $\Sigma$ (or $\Sigma$-ordinary) if $\eta$ is unramified at every place $\mathfrak{P}$ contained in $\Sigma_{p}$. Then the theta lift $\vartheta(\eta)$ of $\eta$ is nearly $p$-ordinary when $\eta$ is ordinary with respect to $\Sigma$. Conversely if $f$ is a nearly $p$-ordinary $p$-stabilised newform with complex multiplication, there exist a totally imaginary quadratic extension $F$ of $F^{+}$satisfying $\left(\operatorname{ord}_{F / F^{+}}\right)$, a $p$-ordinary CM type $\Sigma$ and a $\Sigma$-admissible größencharacter $\eta$ of type $\left(A_{0}\right)$ on $F$ ordinary with respect to $\Sigma$ such that $f$ is described as $f_{\eta}:=\vartheta(\eta)^{p \text {-st }}$. We think that one can verify these facts by looking at the local component at $\mathfrak{p}$ of the automorphic representation $\pi_{f}$ associated to $f$, but later we shall give a brief proof at Proposition A.3 in Appendix A based upon the local study of the Galois representation $V_{f}$ associated to $f$.

2.2. The cyclotomic $p$-adic $L$-function for Hilbert modular cuspforms. We introduce the notion of the cyclotomic $p$-adic $L$-function associated to Hilbert modular cuspforms in this section. We first define the (complex) $p$-optimal periods in Section 2.2.1, and then discuss the $p$-adic $L$-functions after the result of the second-named author Och12 in Section 2.2.2.

Throughout this section $F^{+}$denotes a totally real number field satisfying the following unramifiedness condition:

- $\left(\mathbf{u n r}_{F^{+}}\right)$the prime $p$ does not ramify in $F^{+}$.

In particular $F^{+}$does not contain primitive $p$-th roots of unity.

2.2.1. The p-optimal complex periods. We recall the definition of the p-optimal complex period associated to a normalised eigencuspform $f$ of weight $\kappa$, level $\mathfrak{N}$ and nebentypus $\underline{\varepsilon}$ in this paragraph after Och12, Definition 3.5]. Let $\kappa_{1}^{\max }$ denote the maximum of the integers $\kappa_{1, \tau}$ over $\tau$ in $I_{F^{+}}$, and set $\tilde{f}(x)=f(x)|\operatorname{det}(x)|_{\mathbb{A}_{F^{+}}}^{\kappa_{1}^{\max }+1}$ for every element $x$ of $G\left(\mathbb{A}_{\mathbb{Q}}\right)$. Then we readily see that $\tilde{f}$ is a Hilbert modular eigencuspform of weight $\tilde{\kappa}$, level $\mathfrak{N}$ and 
nebentypus $\underline{\tilde{\varepsilon}}$, where $\tilde{\kappa}$ and $\underline{\tilde{\varepsilon}}$ are defined as

$$
\tilde{\kappa}=\left(\tilde{\kappa}_{1}, \tilde{\kappa}_{2}\right)=\left(\kappa_{1}-\left(\kappa_{1}^{\max }+1\right) \mathrm{t}, \kappa_{2}-\left(\kappa_{1}^{\max }+1\right) \mathrm{t}\right), \underline{\tilde{\varepsilon}}=\left(\varepsilon|\cdot|_{\mathbb{A}_{F^{+}}^{f}}^{2\left(\kappa_{1}^{\max }+1\right)}, \varepsilon_{+}|\cdot|_{\mathbb{A}_{F^{+}}}^{2\left(\kappa^{\max }+1\right)}\right) .
$$

In particular the weight $\tilde{\kappa}$ of $\tilde{f}$ is critical in the sense of Definition 2.3. The cuspform $\tilde{f}$ is sometimes called a critical twist of $f$. For a subalgebra $A$ of $\mathbb{C}$ containing all Hecke eigenvalues associated to $f$, let $\mathfrak{h}_{\tilde{\kappa}}(\mathfrak{N}, \underline{\tilde{\varepsilon}} ; A)$ denote the image of $R\left(\hat{\Gamma}_{1}(\mathfrak{N}), \Delta_{1}(\mathfrak{N})\right) \otimes_{\mathbb{Z}} A$ in $\operatorname{End}_{A}\left(S_{\tilde{\kappa}}(\mathfrak{N}, \underline{\tilde{\varepsilon}} ; A)\right)$. Then, as an element of $\mathfrak{h}_{\tilde{\kappa}}(\mathfrak{N}, \underline{\tilde{\varepsilon}} ; A)$, the Hecke operator $S(\mathfrak{b})$ is naturally identified with $\tilde{\varepsilon}_{+}^{*}(\mathfrak{b})=\varepsilon_{+}^{*}(\mathfrak{b}) \mathcal{N}^{-2\left(\kappa_{1}^{\max }+1\right)}$. Consider a linear map $\lambda_{\tilde{f}}: \mathfrak{h}_{\tilde{\kappa}}(\mathfrak{N}, \tilde{\tilde{\varepsilon}} ; A) \rightarrow A$ which sends the Hecke operator $T(\mathfrak{l})($ resp. $U(\mathfrak{l}))$ to an eigenvalue $\lambda_{\tilde{f}}(\mathfrak{l})$ of $T(\mathfrak{l})(\operatorname{resp} . U(\mathfrak{l}))$ with respect to the eigencuspform $\tilde{f}$ for every prime ideal $\mathfrak{l}$ which does not divide $\mathfrak{N}$ (resp. which divides $\mathfrak{l})$. Note that $\lambda_{\tilde{f}}(\mathfrak{l})$ equals $\mathcal{N} \mathfrak{l}^{-\left(\kappa_{1}^{\max }+1\right)} \lambda_{f}(\mathfrak{l})$ by construction, where $\lambda_{f}(\mathfrak{l})$ is an eigenvalue of $T(\mathfrak{l})$ (or $U(\mathfrak{l})$ if $\mathfrak{l}$ divides $\mathfrak{N}$ ) with respect to $f$.

Let $Y_{1}(\mathfrak{N}) / \mathbb{Q}$ denote the Hilbert-Blumenthal modular variety of level $\mathfrak{N}$. It is an algebraic variety defined over $\mathbb{Q}$ and obtained as the canonical model of the complex analytic variety $Y_{1}(\mathfrak{N})=G(\mathbb{Q}) \backslash G\left(\mathbb{A}_{\mathbb{Q}}\right) / \hat{\Gamma}_{1}(\mathfrak{N}) C_{\mathbf{i}}$. Note that if we choose a set of representatives $\left\{\mathfrak{c}_{i}\right\}_{i=1}^{h}$ of the strict ray class group $\mathrm{Cl}_{F^{+}}^{+}$of $F^{+}$, we can decompose the Hilbert-Blumenthal modular variety $Y_{1}(\mathfrak{N})(\mathbb{C})$ as

$$
Y_{1}(\mathfrak{N})(\mathbb{C})=\bigsqcup_{i=1}^{h} \Gamma_{1}^{i}(\mathfrak{N}) \backslash \mathfrak{h}^{I_{F}+},
$$

where $\mathfrak{h}$ denotes the Poincaré upper half plane and, for each $i$ with $1 \leq i \leq h, \Gamma_{1}^{i}(\mathfrak{N})$ denotes the arithmetic subgroup of $G(\mathbb{Q})$ defined as

$$
\Gamma_{1}^{i}(\mathfrak{N})=G(\mathbb{Q}) \cap\left(\left(\begin{array}{cc}
\mathfrak{c}_{i} & 0 \\
0 & 1
\end{array}\right)^{-1} \Gamma_{1}(\hat{\mathfrak{N}}) C_{\mathbf{i}}\left(\begin{array}{cc}
\mathfrak{c}_{i} & 0 \\
0 & 1
\end{array}\right)\right) .
$$

Corresponding to the decomposition of the Hilbert-Blumenthal modular variety (2.9), the space of cuspforms $S_{\kappa}\left(\hat{\Gamma}_{1}(\mathfrak{N}) ; \mathbb{C}\right)$ of level $\hat{\Gamma}_{1}(\mathfrak{N})$ is also decomposed as

$$
S_{\tilde{\kappa}}\left(\hat{\Gamma}_{1}(\mathfrak{N}) ; \mathbb{C}\right) \cong \bigoplus_{i=1}^{h} S_{\tilde{\kappa}}\left(\Gamma_{1}^{i}(\mathfrak{N}) ; \mathbb{C}\right) ; h \mapsto\left(h_{i}(z)\right)_{1 \leq i \leq h},
$$

where $S_{\tilde{\kappa}}\left(\Gamma_{1}^{i}(\mathfrak{N}) ; \mathbb{C}\right)$ denotes the space of cuspforms of weight $\tilde{\kappa}$ and level $\Gamma_{1}^{i}(\mathfrak{N})$ on $\mathfrak{h}^{I_{F^{+}}}$. See, for example, Och12, Definition 2.5. and Lemma 2.6.] for details on the decomposition (2.10). In the rest of the article, we choose and fix a set of representatives $\left\{\mathfrak{c}_{i}\right\}_{i=1}^{h}$ of $\mathrm{Cl}_{F^{+}}^{+}$ so that the $\mathfrak{p}$-component of each $\mathfrak{c}_{i}$ equals 1 for all the places $\mathfrak{p}$ of $F^{+}$lying above $p$. Next, we define a standard local system $\mathscr{L}(\tilde{\kappa} ; A)$ on $Y_{1}(\mathfrak{N})(\mathbb{C})$ for a subring $A$ of $\mathbb{C}$ satisfying the following condition $(*)$ :

(*) the subring $A$ contains the normal closure of $\mathfrak{r}_{F^{+}}\left[\mathfrak{d}_{F^{+}}^{-1} \mathfrak{c}_{i}^{-1}\right]$ for each $i$ with $1 \leq i \leq h$, where $\mathfrak{d}_{F^{+}}$denotes the absolute different of $F^{+}$.

For each element $\tau$ of $I_{F^{+}}$, let $L\left(\tilde{\kappa}_{\tau} ; A\right)=\bigoplus_{m_{\tau}=0}^{\tilde{\kappa}_{2, \tau}-\tilde{\kappa}_{1, \tau}-1} A X_{\tau}^{m_{\tau}} Y_{\tau}^{\tilde{\kappa}_{2, \tau}-\tilde{\kappa}_{1, \tau}-1-m_{\tau}}$ denote the free $A$-module spanned by all two-variable homogeneous polynomials of degree $\tilde{\kappa}_{2, \tau}-\tilde{\kappa}_{1, \tau}-1$ with coefficients in $A$. Let $g=\left(\begin{array}{ll}a & b \\ c & d\end{array}\right)$ be an element of $G L_{2}\left(F^{+}\right)$such that all conjugate of the matrix component $a, b, c, d$ of $g$ are contained in $A$. Such an element $g$ acts from the 
left on $L\left(\tilde{\kappa}_{\tau} ; A\right)$ by

$$
\begin{aligned}
X_{\tau}^{m_{\tau}} Y_{\tau}^{\tilde{\kappa}_{2, \tau}-\tilde{\kappa}_{1, \tau}-1-m_{\tau}} & \\
& \mapsto \tau\left(\operatorname{det}(g)^{\tilde{\kappa}_{1, \tau}}\right) \cdot\left(\tau(a) X_{\tau}+\tau(c) Y_{\tau}\right)^{m_{\tau}}\left(\tau(b) X_{\tau}+\tau(d) Y_{\tau}\right)^{\tilde{\kappa}_{2, \tau}-\tilde{\kappa}_{1, \tau}-1-m_{\tau}}
\end{aligned}
$$

for each $m_{\tau}$ with $0 \leq m_{\tau} \leq \tilde{\kappa}_{2, \tau}-\tilde{\kappa}_{1, \tau}-1$. Set $L(\tilde{\kappa} ; A)=\bigotimes_{\tau \in I_{F+}} L\left(\tilde{\kappa}_{\tau} ; A\right)$. Then we define the standard local system $\mathscr{L}(\tilde{\kappa} ; A)$ on $Y_{1}(\mathfrak{N})(\mathbb{C})$ as the sheaf of continuous sections of the following covering map:

$$
G(\mathbb{Q}) \backslash G\left(\mathbb{A}_{\mathbb{Q}}\right) \times L(\tilde{\kappa} ; A) / \hat{\Gamma}_{1}(\mathfrak{N}) C_{\mathbf{i}} \rightarrow G(\mathbb{Q}) \backslash G\left(\mathbb{A}_{\mathbb{Q}}\right) / \hat{\Gamma}_{1}(\mathfrak{N}) C_{\mathbf{i}}=Y_{1}(\mathfrak{N})(\mathbb{C}) .
$$

Here we consider that $L(\tilde{\kappa} ; A)$ admits the trivial right action of $\hat{\Gamma}_{1}(\mathfrak{N}) C_{\mathbf{i}}$, and we let $G(\mathbb{Q})$ and $\hat{\Gamma}_{1}(\mathfrak{N}) C_{\mathbf{i}}$ act on $G\left(\mathbb{A}_{\mathbb{Q}}\right) \times L(\tilde{\kappa} ; A)$ diagonally. The Hecke algebra $\mathfrak{h}_{\tilde{\kappa}}(\mathfrak{N}, \underline{\tilde{\varepsilon}} ; A)$ then acts on the Betti cohomology group $H^{d}\left(Y_{1}(\mathfrak{N})(\mathbb{C}), \mathscr{L}(\tilde{\kappa} ; A)\right)$ via the Hecke correspondences. Note also that, via the decomposition (2.9), we can regard the local system $\mathscr{L}(\tilde{\kappa} ; A)$ as the sheaf of continuous sections of

$$
\Gamma_{1}^{i}(\mathfrak{N}) \backslash\left(\mathfrak{h}^{I_{F}+} \times L(\tilde{\kappa} ; A)\right) \rightarrow \Gamma_{1}^{i}(\mathfrak{N}) \backslash \mathfrak{h}^{I_{F^{+}}}
$$

on each connected component $\Gamma_{1}^{i}(\mathfrak{N}) \backslash \mathfrak{h}^{I_{F}}$ of $Y_{1}(\mathfrak{N})(\mathbb{C})$ of $Y_{1}(\mathfrak{N})(\mathbb{C})$.

Next let $\mathbb{Q}_{f}^{\prime}$ be the composite field $\mathbb{Q}_{f} F^{+, \text {gal }}$ and $\mathfrak{r}_{f}^{\prime}$ its ring of integers. Let us denote by $\mathfrak{r}_{f,(p)}^{\prime}$ the localisation of $\mathfrak{r}_{f}^{\prime}$ at the $p$-adic place induced by the specified $p$-adic embedding $\mathbb{Q}_{f}^{\prime} \subset \overline{\mathbb{Q}} \stackrel{\iota_{p}}{\longrightarrow} \overline{\mathbb{Q}}_{p}$. Note that an arbitrary $\mathfrak{r}_{f,(p)}^{\prime}$-algebra satisfies the condition $(*)$ since $\mathfrak{d}_{F^{+}}$ is a $p$-adic unit due to the assumption $\left(\operatorname{unr}_{F^{+}}\right)$, and it is possible to choose representatives $\left\{\mathfrak{c}_{i}\right\}_{i=1}^{h}$ so that they are relatively prime to $p$. Therefore for an arbitrary $\mathfrak{r}_{f,(p)}^{\prime}$-algebra $A$, we can take the maximal $A$-submodule $H^{d}\left(Y_{1}(\mathfrak{N})(\mathbb{C}), \mathscr{L}(\tilde{\kappa} ; A)\right)\left[\lambda_{\tilde{f}}\right]$ of the Betti cohomology $H^{d}\left(Y_{1}(\mathfrak{N})(\mathbb{C}), \mathscr{L}(\tilde{\kappa} ; A)\right)$ (resp. the maximal $A$-submodule $H_{c}^{d}\left(Y_{1}(\mathfrak{N})(\mathbb{C}), \mathscr{L}(\tilde{\kappa} ; A)\right)\left[\lambda_{\tilde{f}}\right]$ of the compactly supported Betti cohomology $\left.H_{c}^{d}\left(Y_{1}(\mathfrak{N})(\mathbb{C}), \mathscr{L}(\tilde{\kappa} ; A)\right)\right)$ on which the Hecke algebra $\mathfrak{h}_{\tilde{\kappa}}(\mathfrak{N}, \tilde{\tilde{\varepsilon}} ; A)$ acts as the multiplication of the eigenvalues at $\tilde{f}$. Now let $\epsilon$ be an element of $\{ \pm 1\}^{I_{F}}$ which we regard as a character defined on the group of connected components of the infinite part $G L_{2}(\mathbb{R})^{I_{F}}$ in $G L_{2}\left(\mathbb{A}_{F^{+}}\right)$. We consider the composite map

$$
H_{c}^{d}\left(Y_{1}(\mathfrak{N})(\mathbb{C}), \mathscr{L}\left(\tilde{\kappa} ; \mathfrak{r}_{f,(p)}^{\prime}\right)\right) \longrightarrow H_{c}^{d}\left(Y_{1}(\mathfrak{N})(\mathbb{C}), \mathscr{L}(\tilde{\kappa} ; \mathbb{C})\right) \longrightarrow H_{c}^{d}\left(Y_{1}(\mathfrak{N})(\mathbb{C}), \mathscr{L}(\tilde{\kappa} ; \mathbb{C})\right)\left[\lambda_{\tilde{f}}\right]^{\epsilon}
$$

where the first map is a natural one and the second map is the projection. As is well known, the group of connected components act on $H_{c}^{d}\left(Y_{1}(\mathfrak{N})(\mathbb{C}), \mathscr{L}(\tilde{\kappa} ; \mathbb{C})\right)$ in a way compatible with the action of $\mathfrak{h}_{\tilde{\kappa}}(\mathfrak{N}, \tilde{\varepsilon} ; \mathbb{C})$, and the $\epsilon$-eigenspace $H_{c}^{d}\left(Y_{1}(\mathfrak{N})(\mathbb{C}), \mathscr{L}(\tilde{\kappa} ; \mathbb{C})\right)\left[\lambda_{\tilde{f}}\right]^{\epsilon}$ of $H_{c}^{d}\left(Y_{1}(\mathfrak{N})(\mathbb{C}), \mathscr{L}(\tilde{\kappa} ; \mathbb{C})\right)\left[\lambda_{\tilde{f}}\right]$ with respect to this action is of dimension one over $\mathbb{C}$ for each $\epsilon$. Thus the image of $H_{c}^{d}\left(Y_{1}(\mathfrak{N})(\mathbb{C}), \mathscr{L}\left(\tilde{\kappa} ; \mathfrak{r}_{f,(p)}^{\prime}\right)\right)$ under the map (2.11) is free of rank one over the discrete valuation ring $\mathfrak{r}_{f,(p)}^{\prime}$ for each $\epsilon$; or in other words, each $H_{c}^{d}\left(Y_{1}(\mathfrak{N})(\mathbb{C}), \mathscr{L}(\tilde{\kappa} ; \mathbb{C})\right)\left[\lambda_{\tilde{f}}\right]^{\epsilon}$ is equipped with an $\mathfrak{r}_{f,(p)}^{\prime}$-integral structure.

We are now ready to associate the Hilbert cuspform $\tilde{f}$ to a cohomology class $[\tilde{f}]$. Let $\left(\tilde{f}_{i}(z)\right)_{1 \leq i \leq h}$ denote the element of $\bigoplus_{i=1}^{h} S_{\tilde{\kappa}}\left(\Gamma_{1}^{i}(\mathfrak{N}) ; \mathbb{C}\right)$ corresponding to $\tilde{f}$ via the decomposition (2.10), and let us consider the vector-valued differential form $\omega_{\tilde{f}}=\left(\omega_{\tilde{f}_{i}}\right)_{1 \leq i \leq h}$ on $Y_{1}(\mathfrak{N})(\mathbb{C})$ defined as

$$
\omega_{\tilde{f}_{i}}=\tilde{f}_{i}\left(z_{\tau}\right) \prod_{\tau \in I_{F+}}\left(X_{\tau}+z_{\tau} Y_{\tau}\right)^{\tilde{\kappa}_{2, \tau}-\tilde{\kappa}_{1, \tau}-1} \bigwedge_{\tau \in I_{F^{+}}} d z_{\tau}
$$


on each connected component $\Gamma_{1}^{i}(\mathfrak{N}) \backslash \mathfrak{h}^{I_{F^{+}}}$of $Y_{1}(\mathfrak{N})(\mathbb{C})$, where $\left(z_{\tau}\right)_{\tau \in I_{F^{+}}}$denotes the standard coordinate of $\mathfrak{h}^{I_{F^{+}}}$. The integration of $\omega_{\tilde{f}}$ on a $d$-cycle of $Y_{1}(\mathfrak{N})(\mathbb{C})$ then defines a cohomology class $[\tilde{f}]$ of $H^{d}\left(Y_{1}(\mathfrak{N})(\mathbb{C}), \mathscr{L}(\tilde{\kappa} ; \mathbb{C})\right)$, which we call the Eichler-Shimura class associated to the critical twist $\tilde{f}$ of $f$. We use the same symbol $[\tilde{f}]$ for its image under the composition

$$
H^{d}\left(Y_{1}(\mathfrak{N})(\mathbb{C}), \mathscr{L}(\tilde{\kappa} ; \mathbb{C})\right) \stackrel{\operatorname{proj}}{\rightarrow} H^{d}\left(Y_{1}(\mathfrak{N})(\mathbb{C}), \mathscr{L}(\tilde{\kappa} ; \mathbb{C})\right)\left[\lambda_{\tilde{f}}\right] \cong H_{c}^{d}\left(Y_{1}(\mathfrak{N})(\mathbb{C}), \mathscr{L}(\tilde{\kappa} ; \mathbb{C})\right)\left[\lambda_{\tilde{f}}\right]
$$

by abuse of notation.

Definition 2.14. For each $\epsilon$, let us choose an $\mathfrak{r}_{f,(p)}^{\prime}$-basis $b^{\epsilon}$ of the image of the rankone free $\mathfrak{r}_{f,(p)}^{\prime}$-module $H_{c}^{d}\left(Y_{1}(\mathfrak{N})(\mathbb{C}), \mathscr{L}\left(\tilde{\kappa} ; \mathfrak{r}_{f,(p)}^{\prime}\right)\right)$ under the map (2.11). Then we define a p-optimal complex period $C_{f, \infty}^{\epsilon} \in \mathbb{C}^{\times}$of signature $\epsilon$ of $f$ to be the constant given by

$$
[\tilde{f}]^{\epsilon}=C_{f, \infty}^{\epsilon} \cdot b^{\epsilon} .
$$

where $[\tilde{f}]^{\epsilon}$ denotes the image of $[\tilde{f}]$ under the projection onto $H^{d}\left(Y_{1}(\mathfrak{N})(\mathbb{C}), \mathscr{L}(\tilde{\kappa} ; \mathbb{C})\right)\left[\lambda_{\tilde{f}}\right]^{\epsilon}$. The complex period $C_{f, \infty}^{\epsilon}$ depends on the choice of $b^{\epsilon}$ which has an ambiguity of a multiple of an element in $\left(\mathfrak{r}_{f,(p)}^{\prime}\right)^{\times}$. Hence we often regard $C_{f, \infty}^{\epsilon}$ as an element of $\mathbb{C}^{\times} /\left(\mathfrak{r}_{f,(p)}^{\prime}\right)^{\times}$.

2.2.2. The cyclotomic p-adic L-functions. We here introduce the notion of the cyclotomic $p$-adic $L$-functions associated to Hilbert modular cuspforms and their interpolation formulae (Theorem 2.15).

Let $\mathrm{Cl}_{F^{+}}^{+}\left(p^{\infty} \mathfrak{r}_{F^{+}}\right)$be the strict ray class group of $F^{+}$modulo $p^{\infty} \mathfrak{r}_{F^{+}}$, which is defined as the projective limit $\mathrm{Cl}_{F^{+}}^{+}\left(p^{\infty} \mathfrak{r}_{F^{+}}\right)=\lim _{\longleftarrow \rightarrow \infty} \mathbb{A}_{F^{+}}^{\times} /\left(F^{+}\right)^{\times} I_{p^{n} \mathfrak{r}_{F^{+}}}$. Here the subgroup $I_{p^{n} \mathfrak{r}_{F^{+}}}$ of $\mathbb{A}_{F^{+}}^{\times}$is defined as

$$
I_{p^{n} \mathfrak{r}_{F^{+}}}=\prod_{\mathfrak{l} p \mathfrak{r}_{F^{+}}} \mathfrak{r}_{F^{+}, \mathfrak{l}}^{\times} \times \prod_{\mathfrak{p} \mid p \mathfrak{r}_{F^{+}}}\left(1+\mathfrak{p}^{n m_{\mathfrak{p}}} \mathfrak{r}_{F^{+}, \mathfrak{p}}\right) \times \prod_{w: \text { archimedean place of } F^{+}}\left(\mathfrak{r}_{F^{+}, w}^{\times}\right)_{+}
$$

when $p \mathfrak{r}_{F^{+}}$is decomposed as $p \mathfrak{r}_{F^{+}}=\prod_{\mathfrak{p} \mid p \mathfrak{r}_{F^{+}}} \mathfrak{p}^{m_{\mathfrak{p}}}$. For an archimedean place $w$, we denote by $\left(\mathfrak{r}_{F^{+}, w}^{\times}\right)_{+}$the connected component of $\mathfrak{r}_{F^{+}, w}^{\times}$containing 1, which is isomorphic to $\mathbb{R}_{>0}$. We denote by $F_{p^{\infty} \mathfrak{r}_{F^{+}}}^{+}$the strict ray class field modulo $p^{\infty} \mathfrak{r}_{F^{+}}$over $F^{+}$, the field corresponding to $\mathrm{Cl}_{F^{+}}^{+}\left(p^{\infty} \mathfrak{r}_{F^{+}}\right)$via global class field theory. For a ray class character $\phi: \mathrm{Cl}_{F^{+}}^{+}\left(p^{\infty} \mathfrak{r}_{F^{+}}\right) \rightarrow \mathbb{C}^{\times}$of finite order, we associate its signature $\operatorname{sgn}(\phi) \in\{ \pm 1\}^{I_{F^{+}}}$in the following manner. Observe that there is a canonical homomorphism from the component group $\{ \pm 1\}^{I_{F^{+}}}$of the archimedean part of the idele class group to $\mathrm{Cl}_{F^{+}}^{+}\left(p^{\infty} \mathfrak{r}_{F^{+}}\right)$. Via this homomorphism, we associate a character $\left(\phi_{\tau}\right)_{\tau \in I_{F^{+}}}$on $\{ \pm 1\}^{I_{F^{+}}}$to $\phi$. We define as $\operatorname{sgn}(\phi)=\left(\phi_{\tau}(-1)\right)_{\tau \in I_{F^{+}}}$.

We next define the Gaussian sum $G(\phi)$ of the ray class character $\phi: \mathrm{Cl}_{F^{+}}^{+}\left(p^{\infty} \mathfrak{r}_{F^{+}}\right) \rightarrow \mathbb{C}^{\times}$ of finite order as

$$
G(\phi)=\sum_{x \in\left(\mathfrak{C}(\phi)^{-1} / \mathfrak{r}_{F^{+}}\right)^{\times}} \phi(x) \exp \left(2 \pi \sqrt{-1} \operatorname{Tr}_{F^{+} / \mathbb{Q}}(x)\right),
$$

where we denote by $\left(\mathfrak{C}(\phi)^{-1} / \mathfrak{r}_{F^{+}}\right)^{\times}$the subset of $\mathfrak{C}(\phi)^{-1} / \mathfrak{r}_{F^{+}}$consisting of elements whose annihilators exactly coincide with $\mathfrak{C}(\phi)$. In the defining equation of $G(\phi)$ we evaluate $\phi$ at an element $x$ of $\left(\mathfrak{C}(\phi)^{-1} / \mathfrak{r}_{F^{+}}\right)^{\times}$via the following composition:

$$
\left(\mathfrak{C}(\phi)^{-1} / \mathfrak{r}_{F^{+}}\right)^{\times} \stackrel{\sim}{\rightarrow}\left(\mathfrak{r}_{F^{+}} / \mathfrak{C}(\phi)\right)^{\times} \rightarrow\left(\mathfrak{r}_{F^{+}} / \mathfrak{C}(\phi)\right)^{\times} / \mathfrak{r}_{F^{+}}^{\times} \hookrightarrow \mathrm{Cl}_{F^{+}}^{+}(\mathfrak{C}(\phi)) .
$$


For a Galois character $\phi: \operatorname{Gal}\left(F_{p^{\infty} \mathfrak{r}_{F^{+}}}^{+} / F^{+}\right) \rightarrow \overline{\mathbb{Q}}^{\times}$of finite order, there exists a unique character on $\mathrm{Cl}_{F^{+}}^{+}\left(p^{\infty} \mathfrak{r}_{F^{+}}\right)$whose value at a prime ideal $\mathfrak{l}$ relatively prime to $p \mathfrak{r}_{F^{+}}$equals $\phi\left(\right.$ Frob $\left._{\mathfrak{l}}^{-1}\right)$, the evaluation of $\phi$ at the arithmetic Frobenius element of $\mathfrak{l}$. By abuse of notation, we use the same symbol $\phi$ for this unique ray class character.

We now state the existence of the cyclotomic $p$-adic $L$-function associated to a Hilbert modular cuspform, which is originally due to Manin [Man76, Sections 5 and 6]. For an element $k$ of $\mathbb{Z}\left[I_{F^{+}}\right]$, we define the integer $k^{\max }$ (resp. $k_{\min }$ ) as the maximum (resp. the minimum) among its coefficients.

Theorem 2.15. Let $f$ be a normalised nearly p-ordinary eigencuspform in $S_{\kappa}(\mathfrak{N}, \underline{\varepsilon} ; \overline{\mathbb{Q}})$ which is stabilised at $p$. We fix a discrete valuation ring $\mathcal{O}$ finite flat over $\mathbb{Z}_{p}$ which contains $\mathfrak{r}_{f,(p)}^{\prime}$. Then there exists an element $\mathcal{L}_{p}^{\text {cyc }}(f)$ of $\mathcal{O}\left[\left[\operatorname{Gal}\left(F^{+}\left(\mu_{p^{\infty}}\right) / F^{+}\right)\right]\right] \otimes_{\mathbb{Z}_{p}} \mathbb{Q}_{p}$ characterised by the interpolation property (recall that we have put $d=\left[F^{+}: \mathbb{Q}\right]$ )

$$
\chi_{p, \text { cyc }}^{j} \phi\left(\mathcal{L}_{p}^{\text {cyc }}(f)\right)=\frac{\Gamma\left(j \mathrm{t}-\kappa_{1}\right)}{\Gamma\left(\left(\kappa_{1}^{\max }+1\right) \mathrm{t}-\kappa_{1}\right)} G(\phi) \prod_{\mathfrak{p} \mid p \mathbf{r}_{F^{+}}} A_{\mathfrak{p}}(f ; \phi, j) \frac{L\left(f, \phi^{-1}, j\right)}{(-2 \pi \sqrt{-1})^{d\left(j-\kappa_{1}^{\max }-1\right)} C_{f, \infty}^{\epsilon_{\phi, j}}}
$$

for an arbitrary integer $j$ satisfying $\kappa_{1}^{\max }+1 \leq j \leq \kappa_{2, \min }$ and an arbitrary character $\phi$ of $\operatorname{Gal}\left(F^{+}\left(\mu_{p^{\infty}}\right) / F^{+}\right)$of finite order. Here $C_{f, \infty}^{\epsilon_{\phi, j}}$ denotes a p-optimal complex period of signature $\epsilon_{\phi, j}$ (see Definition 2.14). The signature $\epsilon_{\phi, j}$ is defined as $(-1)^{j-\kappa_{1}^{\max }-1} \operatorname{sgn}(\phi)$.

The $\mathfrak{p}$-adic multiplier $A_{\mathfrak{p}}(f ; \phi, j)$ is defined as

$$
A_{\mathfrak{p}}(f ; \phi, j)= \begin{cases}1-\frac{\phi(\mathfrak{p}) \mathcal{N} \mathfrak{p}^{j-1}}{\alpha_{\mathfrak{p}}(f)} & \text { if } \mathfrak{p} \text { does not divide } \mathfrak{C}(\phi), \\ \left(\frac{\mathcal{N}_{\mathfrak{p}}^{j-1}}{\alpha_{\mathfrak{p}}(f)}\right)^{\operatorname{ord}_{\mathfrak{p}}(\mathfrak{C}(\phi))} & \text { if } \mathfrak{p} \text { divides } \mathfrak{C}(\phi),\end{cases}
$$

where $\alpha_{\mathfrak{p}}(f)$ denotes the eigenvalue of $f$ with respect to the Hecke operator $U(\mathfrak{p})$. The gamma factor is abbreviated by using multi-indices as

$$
\Gamma\left(m \mathrm{t}-\kappa_{1}\right)=\prod_{\tau \in I_{F^{+}}} \Gamma\left(m-\kappa_{1, \tau}\right) \quad \text { for each } m \text { satisfying } m \geq \kappa_{1}^{\max }+1 .
$$

Remark 2.16. The construction of [Man76] is based upon modular symbols over Hilbert modular varieties, which is a generalisation of the work by Manin himself, Mazur and Swinnerton-Dyer, Višik, Amice and Vélu for elliptic modular case (see [MTT86] for historical reviews). The construction of Theorem 2.15 is revisited by [Di13, Och12] in the context of generalising it into nearly ordinary Hida deformations.

Remark 2.17. There is another fashion of construction of $p$-adic $L$-functions associated to (Hilbert) modular forms which is based upon the theory of Rankin-Selberg convolutions. Methods of the construction of $p$-adic $L$-functions in this direction are discussed by Panchishkin [Pa94], Dabrowski [Da94], Mok [Mok09] and so on. The complex period appearing in their theory is of Shimura type; namely, it is defined by using the self Petersson inner product of the given cuspform.

2.3. Katz, Hida and Tilouine's $p$-adic $L$-functions for CM number fields. In this subsection we introduce the $p$-adic $L$-function (or the $p$-adic measure) for a CM number field which was first constructed by Katz [Katz78] for größencharacters of type $\left(A_{0}\right)$ with 
conductors dividing $p^{\infty}$, and then by Hida and Tilouine [HT93, Theorem II] for general größencharacters of type $\left(A_{0}\right)$.

2.3.1. p-ordinary $C M$ types. As in Introduction, let $F$ be a CM number field of degree $2 d$ with the maximal totally real subfield $F^{+}$. We denote by $c$ the complex conjugation of $F$, that is, the unique generator of the Galois group of $F / F^{+}$. We impose the two assumptions $\left(\operatorname{unr}_{F}^{+}\right)$and $\left(\operatorname{ord}_{F / F^{+}}\right)$on $F / F^{+}$and $p$, which are introduced at the beginning of Section 2.2 and a little bit before Proposition-Definition 2.13 respectively.

By virtue of the assumption ( $\operatorname{ord}_{F / F^{+}}$), one can consider a p-ordinary CM type $\Sigma$ of $F$ (also called a p-adic CM type). Namely $\Sigma$ is a subset of $I_{F}$ satisfying the following two conditions:

- we have $I_{F}=\Sigma \cup \Sigma^{c}$ (disjoint union) where $\Sigma^{c}=\left\{\sigma \circ c \in I_{F} \mid \sigma \in \Sigma\right\}$;

- we have \{places of $F$ lying above $p\}=\Sigma_{p} \cup \Sigma_{p}^{c}$ (disjoint union) where $\Sigma_{p}$ the set of places of $F$ induced by embeddings $\iota_{p} \circ \sigma$ for all $\sigma$ in $\Sigma$ and $\Sigma_{p}^{c}$ the set of their complex conjugates.

It is not difficult to see that there exists a $p$-ordinary CM type $\Sigma$ if and only if the condition $\left(\operatorname{ord}_{F / F^{+}}\right)$is satisfied and that the number of $p$-ordinary CM types is equal to

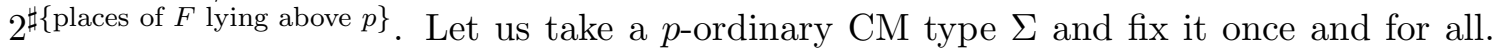
Then, by the definition of größencharacter of type $\left(A_{0}\right)$, the sum $\mu_{\sigma}+\mu_{\bar{\sigma}}$ has the same value for every $\sigma$ in $\Sigma$. We denote this constant by $-w$. The infinity type $\mu$ of $\eta$ thus has two expressions as follows:

$$
\mu=\sum_{\sigma \in \Sigma}\left(\mu_{\sigma} \sigma+\mu_{\bar{\sigma}} \bar{\sigma}\right)=-w \mathrm{t}-\sum_{\sigma \in \Sigma} r_{\sigma}(\sigma-\bar{\sigma}) .
$$

2.3.2. The CM periods of Katz, Hida and Tilouine. We next introduce the complex and $p$-adic periods which appear in the interpolation formula of the $p$-adic $L$-functions for CM number fields.

Let $\mathfrak{C}$ be an integral ideal of $F$ which is prime to $p$ and let us choose and fix an element $\delta$ of $F$ which satisfies the following two conditions:

$\left(1_{\delta}\right)$ the imaginary part $\operatorname{Im}(\sigma(\delta))$ of $\delta$ is positive for all $\sigma$ in $\Sigma$;

$\left(2_{\delta}\right)$ the alternating form defined by $\langle u, v\rangle_{\delta}=\left(u v^{c}-u^{c} v\right) / 2 \delta$ induces an isomorphism between $\mathfrak{r}_{F} \wedge_{\mathfrak{r}_{F^{+}}} \mathfrak{r}_{F}$ and $\mathfrak{d}_{F^{+}}^{-1} \mathfrak{c}^{-1}$ for a certain fractional ideal $\mathfrak{c}$ of $F^{+}$prime to $p \mathfrak{C} \mathfrak{C}^{c}$.

Here $\mathfrak{d}_{F^{+}}$denotes the absolute different of $F^{+}$. We embed $\mathfrak{r}_{F}$ into $\mathbb{C}^{\Sigma}$ diagonally via the fixed (p-ordinary) CM-type $\Sigma$ and denote its image by $\Sigma\left(\mathfrak{r}_{F}\right)$, which becomes a $\mathbb{Z}$-lattice in $\mathbb{C}^{\Sigma}$. We denote the complex torus $\mathbb{C}^{\Sigma} / \Sigma\left(\mathfrak{r}_{F}\right)$ by $X\left(\mathfrak{r}_{F}\right)$. Then the pairing $\langle,\rangle_{\delta}$ defines $a \mathfrak{c}$-polarisation on $X\left(\mathfrak{r}_{F}\right)$ and thus the pair $\left(X\left(\mathfrak{r}_{F}\right),\langle,\rangle_{\delta}\right)$ gives rise to an abelian variety $\left(X\left(\mathfrak{r}_{F}\right), \lambda_{\delta}\right)$ equipped with a $\mathfrak{c}$-polarisation $\lambda_{\delta}: X\left(\mathfrak{r}_{F}\right)^{t} \stackrel{\sim}{\rightarrow} X\left(\mathfrak{r}_{F}\right) \otimes_{\mathfrak{r}_{F}} \mathfrak{c}$. By construction the $\mathfrak{c}$-polarised abelian variety $\left(X\left(\mathfrak{r}_{F}\right), \lambda_{\delta}\right)$ is equipped with complex multiplication by $\mathfrak{r}_{F}$. Note that the element $2 \delta$ is a generator of the fractional ideal $\mathfrak{c} \mathfrak{d}_{F}$ of $F$ by the polarisation condition $\left(2_{\delta}\right)$ (see also Katz78, Lemme (5.7.35)]). In particular, if we embed $2 \delta$ into the idèle group $\mathbb{A}_{F}^{\times} / F^{\times}$diagonally and denote $(2 \delta)_{w}$ the component at the prime $w$, we can take $(2 \delta)_{\mathfrak{Q}}$ as a generator of $\mathfrak{d}_{F} \otimes_{\mathfrak{r}_{F}} \mathfrak{r}_{F, \mathfrak{Q}}$ for every prime ideal $\mathfrak{Q}$ of $F$ relatively prime to $\mathfrak{c}$.

Next we endow $\left(X\left(\mathfrak{r}_{F}\right), \lambda_{\delta}\right)$ with a $\Gamma_{00}\left(p^{\infty}\right)$-level structure. In order to do so, we first decompose $\mathfrak{C}$ into a product $\mathfrak{C}=\mathfrak{F} \mathfrak{F}_{c} \mathfrak{I}$ so that $\mathfrak{F F}_{c}$ is a product of prime ideals completely split over $F^{+}, \mathfrak{I}$ is that of prime ideals inert or ramified over $F^{+}, \mathfrak{F}$ and $\mathfrak{F}_{c}$ are relatively prime and $\mathfrak{F}_{c}^{c}$ (the complex conjugate of $\mathfrak{F}_{c}$ ) contains $\mathfrak{F}$. We fix such a decomposition of $\mathfrak{C}$ once and for all. We put $\mathfrak{f}$ as $\mathfrak{F} \mathfrak{I} \cap F^{+}$and $\mathfrak{f}_{c}$ as $\mathfrak{F}_{c} \mathfrak{I} \cap F^{+}$; then $\mathfrak{f}$ and $\mathfrak{f}_{c}$ are integral ideals of $F^{+}$and $\mathfrak{f}_{c}$ contains $\mathfrak{f}$. We choose a differential idèle $\mathrm{d}_{F^{+}}=\left(\mathrm{d}_{v}\right)_{v}$ of $F^{+}$, a finite idèle 
of $F^{+}$whose associated modulus coincides with $\mathfrak{d}_{F^{+}}$, so that the following conditions are fulfilled:

- the local component $\mathrm{d}_{v}$ equals 1 unless $v$ divides $p \mathfrak{f} \mathfrak{d}_{F^{+}}$;

- if $\mathfrak{Q}$ is a prime divisor of $\mathfrak{F}$ and $\mathfrak{q}$ is the unique prime ideal of $F^{+}$lying below $\mathfrak{Q}$, the local component $d_{\mathfrak{q}}$ of $d$ is given by $(2 \delta)_{\mathfrak{Q}}$, where we identify $F_{\mathfrak{q}}^{+}$with $F_{\mathfrak{Q}}$ via the isomorphism induced by the canonical inclusion $F^{+} \hookrightarrow F$.

Then the composition

$$
\begin{aligned}
\left(\mathfrak{d}_{F^{+}}^{-1} \otimes_{\mathbb{Z}} \mathbb{G}_{m}\right)\left[p^{\infty}\right](\mathbb{C}) & \cong \mathfrak{d}_{F^{+}}^{-1} \prod_{\mathfrak{p} \mid p \mathfrak{r}_{F^{+}}}\left(\mathfrak{p}^{-\infty} / \mathfrak{r}_{F^{+}}\right) \\
& \stackrel{\times \mathfrak{d}_{F^{+}}}{\longrightarrow} \prod_{\mathfrak{p} \mid p \mathfrak{r}_{F^{+}}}\left(\mathfrak{p}^{-\infty} / \mathfrak{r}_{F^{+}}\right) \stackrel{\sim}{\longrightarrow} \prod_{\mathfrak{P} \in \Sigma_{p}}\left(\mathfrak{P}^{-\infty} / \mathfrak{r}_{F}\right) \hookrightarrow \mathbb{C}^{\Sigma} / \Sigma\left(\mathfrak{r}_{F}\right)=X\left(\mathfrak{r}_{F}\right) .
\end{aligned}
$$

induces a $\Gamma_{00}\left(p^{\infty}\right)$-level structure $i\left(\mathfrak{r}_{F}\right):\left(\mathfrak{d}_{F^{+}}^{-1} \otimes_{\mathbb{Z}} \mathbb{G}_{m}\right)\left[p^{\infty}\right] \hookrightarrow X\left(\mathfrak{r}_{F}\right)$ over $\mathbb{C}$. The theory of complex multiplication enables us to find a model of the triple $\left(X\left(\mathfrak{r}_{F}\right), \lambda_{\delta}, i\left(\mathfrak{r}_{F}\right)\right)$ over a valuation ring which is obtained as the inverse image under $\iota_{p}$ of a certain finite integral extension $\widehat{\mathcal{O}}^{\prime}$ of $\widehat{\mathcal{O}}^{\text {ur }}$. Moreover, since we admit the unramifiedness condition $\left(\mathrm{unr}_{F^{+}}\right)$, we can take $\widehat{\mathcal{O}}^{\prime}$ as $\widehat{\mathcal{O}}^{\text {ur }}$ itself due to the fundamental theorem of the theory of complex multiplication combined with Serre and Tate's criterion for good reduction. Let us denote the inverse image of $\widehat{\mathcal{O}}^{\text {ur }}$ under $\iota_{p}$ by $\mathcal{W}$ and take a $\mathfrak{r}_{F} \otimes_{\mathbb{Z}} \mathcal{W}$-basis $\omega\left(\mathfrak{r}_{F}\right)$ of the module of

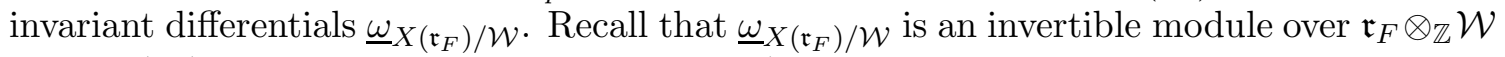
since $X\left(\mathfrak{r}_{F}\right)$ has complex multiplication by $\left.\mathfrak{r}_{F}\right)$.

By construction, $X\left(\mathfrak{r}_{F}\right)$ admits a canonical complex uniformisation $\Pi: \mathbb{C}^{\Sigma} \rightarrow X\left(\mathfrak{r}_{F}\right)$ defined as the quotient with respect to the lattice $\Sigma\left(\mathfrak{r}_{F}\right)$. It induces an isomorphism between the modules of invariant differentials

$$
\Pi^{*}: \underline{\omega}_{X\left(\mathfrak{r}_{F}\right)} \stackrel{\sim}{\rightarrow} \bigoplus_{\sigma \in \Sigma} \mathbb{C} d u_{\sigma}
$$

and we can take $\omega_{\text {trans }}\left(\mathfrak{r}_{F}\right)=\sum_{\sigma \in \Sigma} d u_{\sigma}$ as a $\mathfrak{r}_{F} \otimes_{\mathbb{Z}} \mathbb{C}$-basis of the right hand side. Then we define the complex CM period $\Omega_{\mathrm{CM}, \infty}=\left(\Omega_{\mathrm{CM}, \infty, \sigma}\right) \in\left(\mathfrak{r}_{F} \otimes_{\mathbb{Z}} \mathbb{C}\right)^{\times}=\mathbb{C}^{\times, \Sigma}$ by the following equality:

$$
\Pi^{*} \omega\left(\mathfrak{r}_{F}\right)=\Omega_{\mathrm{CM}, \infty} \omega_{\text {trans }}\left(\mathfrak{r}_{F}\right) .
$$

On the other hand, (the $p$-part of) the $\Gamma_{00}\left(p^{\infty}\right)$-level structure induces an isomorphism $i_{p}:\left(\mathfrak{d}_{F+}^{-1} \otimes_{\mathbb{Z}} \mathbb{G}_{m}\right)^{\wedge} \stackrel{\sim}{\rightarrow} X\left(\mathfrak{r}_{F}\right)^{\wedge}$ between the formal completions along the identity sections over $\widehat{\mathcal{O}}^{\text {ur }}$, and hence we obtain the isomorphism

$$
i_{p}^{*}: \underline{\omega}_{X\left(\mathfrak{r}_{F}\right) / \widehat{\mathcal{O}}^{\mathrm{ur}}} \stackrel{\sim}{\rightarrow} \bigoplus_{\sigma \in \Sigma} \widehat{\mathcal{O}}^{\mathrm{ur}} \frac{d T_{\sigma}}{T_{\sigma}} .
$$

We can take $\omega_{\text {can }}\left(\mathfrak{r}_{F}\right)=\sum_{\sigma \in \Sigma} d T_{\sigma} / T_{\sigma}$ as a $\mathfrak{r}_{F} \otimes_{\mathbb{Z}} \widehat{\mathcal{O}}^{\text {ur }}$-basis of the right hand side, and we define the $p$-adic $C M$ period $\Omega_{\mathrm{CM}, p}=\left(\Omega_{\mathrm{CM}, p, \sigma}\right) \in\left(\mathfrak{r}_{F} \otimes_{\mathbb{Z}} \widehat{\mathcal{O}}^{\text {ur }}\right)^{\times}=\left(\widehat{\mathcal{O}}^{\mathrm{ur}, \times}\right)^{\Sigma}$ by the following equality:

$$
i_{p}^{*} \omega\left(\mathfrak{r}_{F}\right)=\Omega_{\mathrm{CM}, p} \omega_{\text {can }}\left(\mathfrak{r}_{F}\right) .
$$

Remark 2.18. One readily observes by the construction above that, when one replaces $\omega\left(\mathfrak{r}_{F}\right)$ with another $\mathfrak{r}_{F} \otimes_{\mathbb{Z}} \mathcal{W}$-basis of $\underline{\omega}_{X\left(\mathfrak{r}_{F}\right) / \mathcal{W}}$, both $\Omega_{\mathrm{CM}, \infty}$ and $\Omega_{\mathrm{CM}, p}$ are multiplied by the same value contained in $\left(\mathfrak{r}_{F} \otimes_{\mathbb{Z}} \mathcal{W}\right)^{\times}$; therefore the ratio of the pair $\left(\Omega_{\mathrm{CM}, \infty}, \Omega_{\mathrm{CM}, p}\right)$ is well defined independently of the choice of a basis $\omega\left(\mathfrak{r}_{F}\right)$ of $\underline{\omega}_{X\left(\mathfrak{r}_{F}\right) / \mathcal{W}}$. 
2.3.3. The p-adic L-functions for CM fields. In order to state the interpolation formula of the $p$-adic $L$-function for the CM number field $F$, we here introduce the notion of dual größencharacters; for a größencharacter $\eta$ of type $\left(A_{0}\right)$ on $F$, the dual größencharacter $\check{\eta}$ of $\eta$ is defined by $\eta(x) \check{\eta}\left(x^{c}\right)=|x|_{\mathbb{A}_{F}}$ for every $x$ in $\mathbb{A}_{F}^{\times}$. In the language of ideal characters, it is characterised as $\eta^{*}(\mathfrak{A}) \check{\eta}^{*}\left(\mathfrak{A}^{c}\right)=\mathcal{N A}^{-1}$ for every fractional ideal $\mathfrak{A}$ relatively prime to $\mathfrak{C}(\eta)$.

Now we are ready to introduce the results of Katz, Hida and Tilouine.

Theorem 2.19 ([Katz78, Theorem (5.3.0)], [HT93, Theorem II]). Let $p$ be an odd prime number and $F$ a $C M$ field of degree $2 d$ with the maximal totally real subfield $F^{+}$. Assume that $F, F^{+}$and $p$ satisfy both the conditions $\left(\operatorname{unr}_{F^{+}}\right)$and $\left(\operatorname{ord}_{F / F^{+}}\right)$. Let us choose and fix a p-ordinary $C M$ type $\Sigma$ of $F$. Let $\mathfrak{C}=\mathfrak{F}_{\mathfrak{F}_{c}} \mathfrak{l}$ be an integral ideal of $F$ relatively prime to $p$ and $\delta$ a purely imaginary element of $F$ satisfying both the conditions $\left(1_{\delta}\right)$ and $\left(2_{\delta}\right)$ stated at the beginning of Section 2.3.2. Then there exists a unique element $\mathcal{L}_{p, \Sigma}^{\mathrm{KHT}}(F)$ in the Iwasawa algebra $\widehat{\mathcal{O}}^{\mathrm{ur}}\left[\left[\operatorname{Gal}\left(F_{\mathfrak{C} p} / F\right)\right]\right]$, where $F_{\mathfrak{C} p}$ denotes the ray class field modulo $\mathfrak{C} p^{\infty}$ over $F$, satisfying

$$
\begin{aligned}
\frac{\eta^{\mathrm{gal}}\left(\mathcal{L}_{p, \Sigma}^{\mathrm{KHT}}(F)\right)}{\Omega_{\mathrm{CM}, p}^{w+2 r}}=\left(\mathfrak{r}_{F}^{\times}:\right. & \left.\mathfrak{r}_{F^{+}}^{\times}\right) W_{p}(\eta) \frac{(-1)^{w d}(2 \pi)^{|r|} \Gamma_{\Sigma}(w \mathrm{t}+\mathrm{r})}{\sqrt{\left|D_{F^{+}}\right|} \operatorname{Im}(2 \delta)^{\mathrm{r}}} \\
& \times \prod_{\mathfrak{L} \mid \mathfrak{C}}\left(1-\eta^{*}(\mathfrak{L})\right) \prod_{\mathfrak{P} \in \Sigma_{p}}\left\{\left(1-\eta^{*}\left(\mathfrak{P}^{c}\right)\right)\left(1-\check{\eta}^{*}\left(\mathfrak{P}^{c}\right)\right)\right\} \frac{L\left(\eta^{*}, 0\right)}{\Omega_{\mathrm{CM}, \infty}^{w \mathrm{t}+2 r}}
\end{aligned}
$$

for each größencharacter $\eta$ of type $\left(A_{0}\right)$ with conductor dividing $\mathfrak{C} p^{\infty}$ such that

(i) the conductor of $\eta$ is divisible by all prime factors of $\mathfrak{F}$;

(ii) the infinity type $\mu=-w \mathrm{t}-\sum_{\sigma \in \Sigma} r_{\sigma}(\sigma-\bar{\sigma})$ of $\eta$ satisfies either of the followings;

(a) $w \geq 1$ and $r_{\sigma} \geq 0$ for all $\sigma$ in $\Sigma$;

(b) $w \leq 1$ and $w+r_{\sigma}-1 \geq 0$ for all $\sigma$ in $\Sigma$.

The local $\varepsilon$-factor $W_{p}(\eta)$ at $p$ is defined as

$$
W_{p}(\eta)=\prod_{\mathfrak{P} \in \Sigma_{p}} \mathcal{N} \mathfrak{P}^{-e(\mathfrak{P})} \eta_{\mathfrak{P}}\left(\varpi_{\mathfrak{P}}^{-e(\mathfrak{P})}\right) \sum_{x \in\left(\mathfrak{r}_{F, \mathfrak{P}} / \mathfrak{P}^{\left(\mathfrak{P}^{\prime}\right)}\right)^{\times}} \eta_{\mathfrak{P}}(x) e_{\mathfrak{P}}\left(\varpi_{\mathfrak{P}}^{-e(\mathfrak{P})}(2 \delta)_{\mathfrak{P}}^{-1} x\right)
$$

where $\varpi_{\mathfrak{P}}$ denotes a uniformiser of the local field $F_{\mathfrak{P}}$ and $e(\mathfrak{P})$ denotes the exponent of $\mathfrak{P}$ in the conductor of $\eta$. In the equation above we diagonally embed $2 \delta$ into the idèle group $\mathbb{A}_{F}^{\times}$and denote its $\mathfrak{P}$-component by $(2 \delta)_{\mathfrak{P}}$.

In Theorem 2.19 we use the following convention on multi-indices;

$$
\begin{gathered}
\Omega_{\mathrm{CM}, ?}^{w \mathrm{t}+2 \mathrm{r}}=\prod_{\sigma \in \Sigma} \Omega_{\mathrm{CM}, ?, \sigma}^{w+2 r_{\sigma}} \quad \text { for } ?=\mathrm{CM} \text { or } p, \quad|\mathrm{r}|=\sum_{\sigma \in \Sigma} r_{\sigma}, \\
\Gamma_{\Sigma}(w \mathrm{t}+\mathrm{r})=\prod_{\sigma \in \Sigma} \Gamma\left(w+r_{\sigma}\right), \quad \operatorname{Im}(2 \delta)^{\mathrm{r}}=\prod_{\sigma \in \Sigma} \operatorname{Im}(\sigma(2 \delta))^{r_{\sigma}} .
\end{gathered}
$$

Remark 2.20. The $p$-adic $L$-function $\mathcal{L}_{p, \Sigma}^{\mathrm{KHT}}(F)$ does depend on the choice of $\delta$ satisfying the conditions $\left(1_{\delta}\right)$ and $\left(2_{\delta}\right)$, but we can explicitly describe effects on the interpolation formula when we replace $\delta$ by another purely imaginary element $\delta^{\prime}$ satisfying the polarisation conditions $\left(1_{\delta}\right)$ and $\left(2_{\delta}\right)$. In particular, we readily observe that the $p$-adic valuation of $\mathcal{L}_{p, \Sigma}^{\mathrm{KHT}}(F)$ does not change after such a replacement of $\delta$. Refer, for example, to Katz78, Section 5.8]. We also remark that the assumption $\left(u_{F_{F}}\right)$ is not required in Katz, Hida and Tilouin's original construction of $\mathcal{L}_{p, \Sigma}^{\mathrm{KHT}}(F)$. However, without $\left(\mathrm{unr}_{F^{+}}\right)$, the constructed 
$p$-adic $L$-function $\mathcal{L}_{p, \Sigma}^{\mathrm{KHT}}(F)$ might not be an element of $\widehat{\mathcal{O}}^{\mathrm{ur}}\left[\left[\mathrm{Gal}\left(F_{\mathfrak{C} p} / F\right)\right]\right]$ but an element of $\mathcal{O}_{\mathbb{C}_{p}}\left[\left[\operatorname{Gal}\left(F_{\mathfrak{C} p} \infty / F\right)\right]\right]$.

2.4. Comparison of the $p$-adic $L$-functions. In this subsection we specialise Katz, Hida and Tilouine's $p$-adic $L$-function of $\mathcal{L}_{p, \Sigma}^{\mathrm{KHT}}(F)$ for a CM number field to a fixed arithmetic weight parametrisation and obtain an element of the cyclotomic Iwasawa algebra which interpolates critical values of the $L$-function associated to a Hilbert modular form with complex multiplication. At the end of this subsection, we compare the specialised element with the $p$-adic $L$-function $\mathcal{L}_{p}^{\text {cyc }}(f)$ constructed by the second-named author, and formulate a certain conjecture on the relation between Katz's complex CM periods and modular symbolic complex periods.

2.4.1. Comparison of the interpolation formulae. Let the notation be as in the previous subsection. Recall that, in particular, $\eta$ denotes a $\Sigma$-admissible größencharacter of type $\left(A_{0}\right)$ on $F$ ) for an appropriate $p$-ordinary CM type $\Sigma$. Now assume that $\vartheta(\eta)$ is a primitive form; in particular, $\vartheta(\eta)$ is an eigenvector with respect to the Hecke operator $T(\mathfrak{p})$ (or $U(\mathfrak{p}))$ for each $\mathfrak{p}$ lying above $p$. Then, as we have already seen at the end of Section 2.1.5, the cuspform $\vartheta(\eta)$ is nearly $p$-ordinary if and only if $\eta$ is ordinary with respect to $\Sigma$, that is, $\eta$ is unramified at every $\mathfrak{P}$ contained in $\Sigma_{p}$. Assume that $\eta$ is ordinary with respect to $\Sigma$ and let $\vartheta(\eta)^{p \text {-st }}$ denote the $p$-stabilisation of $\vartheta(\eta)$. In particular, the eigenvalue with respect to the normalised Hecke operator $U_{0}(\mathfrak{p})$ is given by $\left\{\mathfrak{p}^{\kappa_{1}}\right\}^{-1} \eta^{*}(\mathfrak{P})$ for each prime ideal $\mathfrak{p}$ lying above $p$; here $\mathfrak{P}$ is an element of $\Sigma_{p}$ satisfying $\mathfrak{P P}^{c}=\mathfrak{p}$. One readily observes that the $p$-adic valuation of $\left\{\mathfrak{p}^{\kappa_{1}}\right\}^{-1} \eta^{*}(\mathfrak{P})$ coincides with that of the evaluation of the $p$-adic avatar $\hat{\eta}$ introduced in Section 2.1.1 at a uniformiser $\varpi_{\mathfrak{P}}$ of $F_{\mathfrak{P}}$. Since the $p$-adic avatar $\hat{\eta}$ takes values into $p$-adic units, we see that $\vartheta(\eta)^{p \text {-st }}$ is indeed nearly ordinary at $p$.

Proposition 2.21. Assume that we have chosen and fixed an element $\delta$ of $F$ satisfying the conditions $\left(1_{\delta}\right)$ and $\left(2_{\delta}\right)$ in Section 2.3.2. Then under the settings and the notation as above, there exists an element $\mathcal{L}_{p, \mathrm{CM}}^{\mathrm{cyc}}(\eta)$ of $\mathcal{O}_{\mathbb{C}_{p}}\left[\left[\mathrm{Gal}\left(F^{+}\left(\mu_{p} \infty\right) / F^{+}\right)\right]\right]$characterised by the interpolation property

$$
\chi_{p, \mathrm{cyc}}^{j} \phi\left(\mathcal{L}_{p, \mathrm{CM}}^{\mathrm{cyc}}(\eta)\right)=\Gamma\left(j \mathrm{t}-\kappa_{\mu, 1}\right) G(\phi) \frac{\prod_{\mathfrak{p} \mid p \mathfrak{r}_{F}} A_{\mathfrak{p}}\left(\vartheta(\eta)^{p-s t}, \phi, j\right) L\left(\vartheta(\eta)^{p-s t}, \phi^{-1}, j\right)}{(-2 \pi \sqrt{-1})^{j d}\left(-\widetilde{\Omega}_{\mathrm{CM}, \infty}\right)^{\kappa_{\mu, 2}}\left(-\Omega_{\mathrm{CM}, \infty}\right)^{-\kappa_{\mu, 1}}}
$$

for an arbitrary natural number $j$ satisfying $\kappa_{\mu, 1}^{\max }+1 \leq j \leq\left(\kappa_{\mu, 2}\right)_{\min }$ and an arbitrary character $\phi$ of finite order of $\operatorname{Gal}\left(F^{+}\left(\mu_{p^{\infty}}\right) / F^{+}\right)$. Here $\Omega_{\mathrm{CM}, \infty}$ denotes Katz's complex $C M$ period introduced in Section 2.3 .2 and $\widetilde{\Omega}_{\mathrm{CM}, \infty}$ denotes the modified complex CM period defined by $\left((2 \delta)^{-1} \otimes 2 \pi \sqrt{-1}\right)^{-1} \Omega_{\mathrm{CM}, \infty}$, which we regard as an element of $\left.\left(F^{+} \otimes \mathbb{C}\right)^{\times}\right)$. The Gaussian sum $G\left(\phi^{-1}\right)$ is defined as (2.13), and the $\mathfrak{p}$-adic multiplier $A_{\mathfrak{p}}\left(\vartheta(\eta)^{p-s t}, \phi, j\right)$ is defined by

$$
A_{\mathfrak{p}}\left(\vartheta(\eta)^{p-s t}, \phi, j\right)= \begin{cases}1-\frac{\phi(\mathfrak{p}) \mathcal{N} \mathfrak{p}^{j-1}}{\eta^{*}(\mathfrak{P})} & \text { if } \mathfrak{p} \text { does not divide } \mathfrak{C}(\phi), \\ \left(\frac{\mathcal{N} \mathfrak{p}^{j-1}}{\eta^{*}(\mathfrak{P})}\right)^{\text {ord }}(\mathfrak{C}(\phi)) & \text { if } \mathfrak{p} \text { divides } \mathfrak{C}(\phi) .\end{cases}
$$

Remark 2.22. Prasanna and the second-named author have already constructed an object corresponding to $\mathcal{L}_{p, \mathrm{CM}}^{\mathrm{cyc}}(\eta)$ in elliptic modular cases $[\mathrm{OP}$, Theorem 2.4], and our modified complex CM period $\widetilde{\Omega}_{\mathrm{CM}, \infty}$ is a counterpart of the CM period $\Omega_{\infty}^{\mathrm{CM}}$ defined there. 
We firstly prepare two elementary lemmata required for the construction of the $p$-adic measure $\mathcal{L}_{p, \mathrm{CM}}^{\mathrm{cyc}}(\eta)$.

Lemma 2.23. The index $\left(\mathfrak{r}_{F}^{\times}: \mathfrak{r}_{F^{+}}^{\times}\right)$is relatively prime to $p$.

Proof. Let $W_{F}$ (resp. $W_{F^{+}}$) denote the group of roots of unity contained in $F$ (resp. in $\left.F^{+}\right)$. Then we have $\left(\mathfrak{r}_{F}^{\times}: \mathfrak{r}_{F^{+}}^{\times}\right)=Q_{F}\left(W_{F}: W_{F^{+}}\right)$where $Q_{F}$ is the Hasse's unit index defined as $\left(\mathfrak{r}_{F}^{\times}: W(F) \mathfrak{r}_{F^{+}}^{\times}\right)$. It is known that $Q_{F}$ can take only two possible values 1 or 2 (see [Ha85, Satz 14] or [Wa97, Theorem 4.12]). In particular $Q_{F}$ is not divisible by $p$ because we assume that $p$ is odd. Moreover the unit group $\mathfrak{r}_{F}^{\times}$contains none of the $p$-power roots of unity since $F / \mathbb{Q}$ is unramified at every place lying above $p$ due to the assumptions $\left(\operatorname{unr}_{F^{+}}\right)$and $\left(\operatorname{ord}_{F / F^{+}}\right)$. Hence the index $\left(W_{F}: W_{F^{+}}\right)$is not divisible by $p$ either. This completes the proof.

Lemma 2.24. Let $\delta$ be an element of $F$ satisfying the conditions $\left(1_{\delta}\right)$ and $\left(2_{\delta}\right)$ at the beginning of Section 2.3.2. Then the product $\prod_{\sigma \in \Sigma} \sigma(2 \delta)$ is an element of $\mathbb{Z}_{p}^{\times}$.

Proof. Recall that $2 \delta$ is a generator of the $p$-part of the absolute discriminant of $F$, or in other words, $2 \delta\left(\mathfrak{r}_{F} \otimes_{\mathbb{Z}} \mathbb{Z}_{p}\right)=\mathfrak{d}_{F} \otimes_{\mathbb{Z}} \mathbb{Z}_{p}$ holds (refer to [Katz78, Lemma (5.7.35)]). The assumptions $\left(\mathrm{unr}_{F^{+}}\right)$and $\left(\operatorname{ord}_{F / F^{+}}\right)$imply that $\mathfrak{d}_{F} \otimes_{\mathbb{Z}} \mathbb{Z}_{p}$ is trivial, and hence $2 \delta$ is a $p$-adic unit; that is, the image of $2 \delta$ in $F \otimes_{\mathbb{Z}} \mathbb{Z}_{p}$ is contained in $\left(\mathfrak{r}_{F} \otimes_{\mathbb{Z}} \mathbb{Z}_{p}\right)^{\times}$. Then the image of $2 \delta$ under the composition

$$
\left(\mathfrak{r}_{F} \otimes_{\mathbb{Z}} \mathbb{Z}_{p}\right)^{\times} \stackrel{\text { projection }}{\longrightarrow} \prod_{\mathfrak{P} \in \Sigma_{p}} \mathfrak{r}_{F, \mathfrak{P}}^{\times} \stackrel{\sim}{\longrightarrow}\left(\mathfrak{r}_{F^{+}} \otimes_{\mathbb{Z}} \mathbb{Z}_{p}\right)^{\times} \stackrel{\mathrm{Nr}_{F^{+} / \mathbb{Q}} \otimes \mathrm{id}}{\longrightarrow} \mathbb{Z}_{p}^{\times}
$$

coincides with $\prod_{\sigma \in \Sigma} \sigma(2 \delta)$ by definition, where the middle isomorphism in the diagram above is the identification induced by the fixed $p$-ordinary CM type $\Sigma$ :

$$
\mathfrak{r}_{F^{+}} \otimes_{\mathbb{Z}} \mathbb{Z}_{p} \stackrel{\sim}{\rightarrow} \prod_{\mathfrak{P} \in \Sigma_{p}} \mathfrak{r}_{F, \mathfrak{P}} ; \quad x \otimes 1 \mapsto\left(\iota_{p} \circ \sigma(x)\right)_{\iota_{p} \circ \sigma \in \Sigma_{p}} .
$$

Therefore $\prod_{\sigma \in \Sigma} \sigma(2 \delta)$ is an element of $\mathbb{Z}_{p}^{\times}$.

Taking the bijection $\Sigma \stackrel{\sim}{\rightarrow} I_{F^{+}} ;\left.\sigma \mapsto \sigma\right|_{F^{+}}$into account, we abbreviated the product $\prod_{\sigma \in \Sigma} \sigma(2 \delta)$ as $(2 \delta)^{\mathrm{t}}$ in the following arguments.

We now explain how to construct the $p$-adic measure $\mathcal{L}_{p, \mathrm{CM}}^{\mathrm{cyc}}(\eta)$. Let $\mathfrak{C}$ denote the primeto- $p$ part of the conductor of $\eta$ and consider Katz, Hida and Tilouine's measure $\mathcal{L}_{p, \Sigma}^{\mathrm{KHT}}(F)$ introduced in Theorem 2.19, which is by construction an element of $\widehat{\mathcal{O}}^{\mathrm{ur}}\left[\left[\operatorname{Gal}\left(F_{\mathfrak{C} p} p^{\infty} / F\right)\right]\right]$. Define a $p$-adic integer $\tilde{d}=\tilde{d}(\delta)$ to be $(-1)^{d}(2 \delta)^{\mathrm{t}}=\prod_{\sigma \in \Sigma} \sigma(-2 \delta)$, which is indeed a $p$-adic unit in $\mathbb{Z}_{p}^{\times}$by Lemma 2.24. Hence there exists a unique element $z_{\tilde{d}}$ of $\operatorname{Gal}\left(F\left(\mu_{p^{\infty}}\right) / F\right)$ corresponding to $\tilde{d}$ via $\chi_{p, \text { cyc }}$.

Let $\sigma_{0}$ denote an element of $\Sigma$ such that $\mu_{\sigma}\left(=\kappa_{\mu, 1,\left.\sigma\right|_{F^{+}}}\right)$takes the maximum $\kappa_{\mu, 1}^{\max }$, and let $\mathfrak{P}_{0}$ denotes the corresponding element of $\Sigma_{p}$ : the prime ideal of $F$ induced by the embedding $\iota_{p} \circ \sigma_{0}: F \hookrightarrow \overline{\mathbb{Q}}_{p}$. We denote the lay class field modulo $\mathfrak{C} \prod_{\mathfrak{P}^{*} \in\left(\Sigma_{p} \cup \Sigma_{p}^{c}\right) \backslash\left\{\mathfrak{P}_{0}\right\}}\left(\mathfrak{P}^{*}\right)^{\infty}$ over $F$ by $F_{\mathfrak{C} p^{\infty}}^{(0)}$. Since $\eta$ is ordinary with respect to $\Sigma$, the $p$-adic avatar of $\eta|\cdot|_{\mathbb{A}_{F}}^{\kappa_{\mu, 1}^{\max }}$ is unramified at $\mathfrak{P}_{0}$ by its construction, and therefore the corresponding Galois character $\eta^{\text {gal }} \chi_{p, \text { cyc }}^{\kappa_{\mu, 1}^{\max }}$ factors through the Galois group $\operatorname{Gal}\left(F_{\mathfrak{C} p^{\infty}}^{(0)} / F\right)$. The ray class field $F_{\mathfrak{C} p^{\infty}}^{(0)}$ is contained in $F_{\mathfrak{C} p^{\infty}}$ by definition. On the other hand, the $p$-adic cyclotomic extension $F\left(\mu_{p^{\infty}}\right) / F$ is totally ramified at $\mathfrak{P}$ since $F$ does not contain any $p$-power root of unity due to the conditions 
$\left(\operatorname{unr}_{F^{+}}\right)$and $\left(\operatorname{ord}_{F / F^{+}}\right)$. By compairing ramification, we see that $F_{\mathfrak{C} p^{\infty}}^{(0)}$ is linearly disjoint from $F\left(\mu_{p^{\infty}}\right)$ over $F$. The Galois group of $F\left(\mu_{p^{\infty}}\right) F_{\mathfrak{C} p^{\infty}}^{(0)} / F$ is thus decomposed as the direct product of $\operatorname{Gal}\left(F\left(\mu_{p^{\infty}}\right) / F\right)$ and $\operatorname{Gal}\left(F_{\mathfrak{C} p^{\infty}}^{(0)} / F\right)$. Via this decomposition we regard $\left(z_{\tilde{d}}, 1\right)$ as an element of $\operatorname{Gal}\left(F\left(\mu_{p^{\infty}}\right) F_{\mathfrak{C} p^{\infty}}^{(0)} / F\right)$ and let $\tilde{z}_{\tilde{d}}$ denote its arbitrary lift to $\operatorname{Gal}\left(F_{\mathfrak{C} p^{\infty}} / F\right)$. Then we define $\mathcal{L}_{p, \mathrm{CM}}^{\text {cyc }}(\eta) \in \widehat{\mathcal{O}}^{\text {ur }}\left[\left[\operatorname{Gal}\left(F^{+}\left(\mu_{p^{\infty}}\right) / F^{+}\right)\right]\right]$as the product of a power of Katz's $p$-adic CM period $\Omega_{\mathrm{CM}, p}^{-\left(\kappa_{\mu, 2}-\kappa_{\mu, 1}\right)}$ and the image of the element $\operatorname{Ex}(F, \delta) \mathcal{L}_{p, \Sigma}^{\mathrm{KHT}}(F)$ under the map

$$
\widehat{\mathcal{O}}^{\text {ur }}\left[\left[\operatorname{Gal}\left(F_{\mathfrak{C} p^{\infty}} / F\right)\right]\right] \rightarrow \widehat{\mathcal{O}}^{\mathrm{ur}}\left[\left[\operatorname{Gal}\left(F\left(\mu_{p^{\infty}}\right) / F\right)\right]\right] \stackrel{\sim}{\rightarrow} \widehat{\mathcal{O}}^{\text {ur }}\left[\left[\operatorname{Gal}\left(F^{+}\left(\mu_{p^{\infty}}\right) / F^{+}\right)\right]\right]
$$

defined as $\left.g \mapsto \eta^{\text {gal }}(g) g\right|_{F^{+}\left(\mu_{p} \infty\right)}$ for each element $g$ of $\operatorname{Gal}\left(F_{\mathfrak{C} p^{\infty}} / F\right)$. Here $\operatorname{Ex}(F, \delta)$ denotes an element defined by the product $\left(\mathfrak{r}_{F}^{\times}: \mathfrak{r}_{F^{+}}^{\times}\right)^{-1} \sqrt{\left|D_{F^{+}}\right|}(-2 \delta)^{-\kappa_{\mu, 1}^{\max }} \tilde{z}_{\tilde{d}(\delta)}^{-1}$, which we call the extra factor. Note that $\mathcal{L}_{p, \mathrm{CM}}^{\mathrm{cyc}}(\eta)$ is indeed $p$-adically integral since $\operatorname{Ex}(F, \delta)$ is a $p$-adic unit by virtue of Lemma 2.23, Lemma 2.24 and the assumption $\left(\mathrm{unr}_{F^{+}}\right)$.

Proof of Proposition 2.21. We shall deduce the desired interpolation formula of $\mathcal{L}_{p, \mathrm{CM}}^{\mathrm{cyc}}(\eta)$ from the interpolation formula of $\mathcal{L}_{p, \Sigma}^{\mathrm{KHT}}(F)$ (see Theorem 2.19). Note that the evaluation of $\mathcal{L}_{p, \mathrm{CM}}^{\mathrm{cyc}}(\eta)$ at $\chi_{p, \text { cyc }}^{j} \phi$ is exactly the same as the evaluation of $\operatorname{Ex}(F, \delta) \mathcal{L}_{p, \Sigma}^{\mathrm{KHT}}(F)$ at the character $\eta^{\text {gal }} \chi_{p, \text { cyc }}^{j} \phi$ by definition.

- Extra factor. - The evaluation of the extra factor $\operatorname{Ex}(F, \delta)$ at the character $\eta^{\text {gal }} \chi_{p, \text { cyc }}^{j} \phi$ is calculated as

$$
\begin{aligned}
\eta^{\text {gal }} \chi_{p, \text { cyc }}^{j} \phi(\operatorname{Ex}(F, \delta)) & =\left(\mathfrak{r}_{F}^{\times}: \mathfrak{r}_{F}^{\times}\right)^{-1} \sqrt{\left|D_{F^{+}}\right|}(-2 \delta)^{-\kappa_{\mu, 1}^{\max }} \eta^{\text {gal }} \chi_{p, \mathrm{cyc}}^{j} \phi\left(\tilde{z}_{\tilde{d}}^{-1}\right) \\
& =\left(\mathfrak{r}_{F}^{\times}: \mathfrak{r}_{F^{+}}^{\times}\right)^{-1} \sqrt{\left|D_{F^{+}}\right|}(-2 \delta)^{-\kappa_{\mu, 1}^{\max }} \chi_{p, \mathrm{cyc}}^{j-\kappa_{\mu, 1}^{\max }} \phi\left(z_{\tilde{d}}^{-1}\right) \eta^{\mathrm{gal}} \chi_{p, \mathrm{cyc}}^{\kappa_{\mu, 1}^{\max }}(1) \\
& =\left(\mathfrak{r}_{F}^{\times}: \mathfrak{r}_{F^{+}}^{\times}\right)^{-1} \sqrt{\left|D_{F^{+}}\right|} \phi\left(z_{\tilde{d}}\right)^{-1}(-2 \delta)^{-j \mathrm{t}} .
\end{aligned}
$$

At the second equality, we just replace $\tilde{z}_{\tilde{d}}$ by the corresponding element $\left(z_{\tilde{d}}, 1\right)$ in the $\operatorname{product} \operatorname{Gal}\left(F\left(\mu_{p^{\infty}}\right) / F\right) \times \operatorname{Gal}\left(F_{\mathfrak{C} p^{\infty}}^{(0)} / F\right)$.

- Interpolation region. - The infinity type $\mu_{j}$ of the größencharacter $\eta^{\text {gal }} \chi_{p \text {,cyc }}^{j} \phi$ is given by $\sum_{\sigma \in \Sigma}\left(\left(\mu_{\sigma}-j\right) \sigma+\left(\mu_{\bar{\sigma}}-j\right) \bar{\sigma}\right)$. We then define integers $w_{j}$ and $r_{j, \sigma}$ by the equation

$$
\mu_{j}=-w_{j} \mathrm{t}-\sum_{\sigma \in \Sigma} r_{j, \sigma}(\sigma-\bar{\sigma}) .
$$

More concretely $w_{j}$ and $r_{j, \sigma}$ are defined as follows:

$$
w_{j}=2 j-\left[\kappa_{\mu}\right] \quad \text { and } \quad r_{j, \sigma}=\mu_{\bar{\sigma}}-j=\kappa_{\mu, 2,\left.\sigma\right|_{F^{+}}}-j \text { for each } \sigma \text { in } \Sigma .
$$

The interpolation region of Katz, Hida and Tilouine's measure for größencharacters of the form $\eta^{\text {gal }} \chi_{p, \text { cyc }}^{j} \phi$ is given by

$$
\left(w_{j} \geq 1 \text { and } r_{j, \sigma} \geq 0 \quad{ }^{\forall} \sigma \in \Sigma\right) \text { or }\left(w_{j} \leq 1 \text { and } r_{j, \sigma}+w_{j}-1 \geq 0 \quad{ }^{\forall} \in \Sigma\right) .
$$

The solution of the simultaneous inequalities above with respect to $j$ is then calculated as $\max \left\{\mu_{\sigma} \mid \sigma \in \Sigma\right\}+1 \leq j \leq \min \left\{\mu_{\bar{\sigma}} \mid \sigma \in \Sigma\right\}$, which coincides with the desired interpolation region $\kappa_{\mu, 1}^{\max }+1 \leq j \leq\left(\kappa_{\mu, 2}\right)_{\text {min }}$ by the definition of $\kappa_{\mu}=\left(\kappa_{\mu, 1}, \kappa_{\mu, 2}\right)$. 
- Periods. - The equality $\Omega_{\mathrm{CM}, ?}^{w_{j} \mathrm{t}+2 \mathrm{r}_{j}}=\Omega_{\mathrm{CM}, ?}^{\kappa_{\mu, 2}-\kappa_{\mu, 1}}$ holds for $\mathrm{r}_{j}=\sum_{\sigma \in \Sigma} r_{j, \sigma} \sigma$ and $?=\infty, p$. In particular the contribution of the $p$-adic $\mathrm{CM}$ period appearing in the interpolation formula of $\mathcal{L}_{p, \Sigma}^{\mathrm{KHT}}(F)$ is canceled by the construction of $\mathcal{L}_{p, \mathrm{CM}}^{\mathrm{cyc}}(\eta)$. Moreover we obtain

$$
\frac{1}{\Omega_{\mathrm{CM}, \infty}^{w_{j} \mathrm{t}+\mathrm{r}_{j}}}=\frac{1}{\Omega_{\mathrm{CM}, \infty}^{\kappa_{\mu, 2}-\kappa_{\mu, 1}}}=\frac{(-1)^{\left[\kappa_{\mu}\right] d}(-2 \delta)^{\kappa_{\mu, 2}}}{(-2 \pi \sqrt{-1})^{\left|\kappa_{\mu, 2}\right|}} \cdot \frac{1}{\left(-\widetilde{\Omega}_{\mathrm{CM}, \infty}\right)^{\kappa_{\mu, 2}}\left(-\Omega_{\mathrm{CM}, \infty}\right)^{-\kappa_{\mu, 1}}} .
$$

- L-value. - First note that the Galois character $\eta^{\text {gal }} \chi_{p, \mathrm{CM}}^{j} \phi$ corresponds to the ideal character $\eta^{*} \mathcal{N}^{-j} \phi^{-1}$ (see the paragraphs before Theorem 2.15). Here we define $\phi^{-1}(\mathfrak{A})$ for a fractional ideal $\mathfrak{A}$ of $F$ by $\phi^{-1}\left(\mathcal{N}_{F / F^{+}} \mathfrak{A}\right)$. The größencharacter $\eta$ is ramified at every prime ideal $\mathfrak{L}$ dividing $\mathfrak{C}$ since $\mathfrak{C}$ is by definition the prime-to- $p$ part of the conductor of $\eta$, and hence the local term $1-\eta^{*} \mathcal{N}^{-j} \phi^{-1}(\mathfrak{L})$ at such $\mathfrak{L}$ equals 1 . By comparing the Dirichlet series expressions, we can readily check that the $L$-value $L\left(\eta^{*} \mathcal{N}^{-j} \phi^{-1}, 0\right)$ exactly equals $L\left(\vartheta(\eta), \phi^{-1}, j\right)$ by the construction of the theta lift. For each place $\mathfrak{p}$ of $F^{+}$lying above $p$, the local factor of $L\left(\vartheta(\eta), \phi^{-1}, j\right)$ at $\mathfrak{p}$ is given by

$$
\begin{aligned}
L_{\mathfrak{p}}(\vartheta(\eta), \phi, j) & =\left\{1-C(\vartheta(\eta), \mathfrak{p}) \phi^{-1}(\mathfrak{p}) \mathcal{N p}^{-j}+\eta^{*}\left(\mathfrak{p r} \mathfrak{r}_{F}\right) \nu_{F / F^{+}}^{*} \phi^{-2}(\mathfrak{p}) \mathcal{N} \mathfrak{p}^{-2 j}\right\}^{-1} \\
& =\left(1-\eta^{*} \phi^{-1}(\mathfrak{P}) \mathcal{N} \mathfrak{P}^{-j}\right)^{-1}\left(1-\eta^{*} \phi^{-1}\left(\mathfrak{P}^{c}\right)\left(\mathcal{N} \mathfrak{P}^{c}\right)^{-j}\right)^{-1}
\end{aligned}
$$

for $\mathfrak{p}=\mathfrak{P P}^{c}$ with $\mathfrak{P}$ in $\Sigma_{p}$. Therefore the equality

$$
\left(1-\eta^{*} \phi^{-1}\left(\mathfrak{P}^{c}\right)\left(\mathcal{N} \mathfrak{P}^{c}\right)^{-j}\right) L_{\mathfrak{p}}(\vartheta(\eta), \phi, j)=\left(1-\eta^{*} \phi^{-1}(\mathfrak{P}) \mathcal{N} \mathfrak{P}^{-j}\right)^{-1}
$$

holds and the right hand side of this equation is no other than the local component at $\mathfrak{p}$ of the $L$-value $L\left(\vartheta(\eta)^{p \text {-st }}, \phi, j\right)$ by the definition of the $p$-stabilisation of $\vartheta(\eta)$. Finally we obtain by the definition of the dual größencharacter an equation

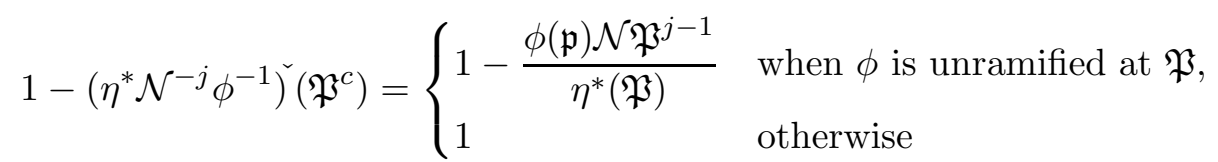

for each $\mathfrak{P}$ in $\Sigma_{p}$ (recall that $\eta^{*} \mathcal{N}^{-j}$ is unramified at $\mathfrak{P}$ due to the assumption $\left(\operatorname{unr}_{F^{+}}\right)$), and it coincides with the $\mathfrak{p}$-adic multiplier $A_{\mathfrak{p}}\left(\vartheta(\eta)^{p \text {-st }} ; \phi, j\right)$ when $\mathfrak{p}=\mathcal{N}_{F / F+\mathfrak{P}}$ does not divide the conductor $\mathfrak{C}(\phi)$ of $\phi$. Consequently we have

$$
\begin{aligned}
& \prod_{\mathfrak{L} \mid \mathfrak{C}}\left(1-\eta^{*} \mathcal{N}^{-j} \phi^{-1}(\mathfrak{L})\right) \prod_{\mathfrak{P} \mid \Sigma_{p}}\left\{\left(1-\eta^{*} \mathcal{N}^{-j} \phi^{-1}\left(\mathfrak{P}^{c}\right)\right)\left(1-\left(\eta^{*} \mathcal{N}^{-j} \phi^{-1}\right)^{\check{(}}\left(\mathfrak{P}^{c}\right)\right)\right\} L\left(\eta^{*} \mathcal{N}^{-j} \phi^{-1}, 0\right) \\
& =\prod_{\mathfrak{p} \mid p \mathfrak{r}_{F^{+}}, \mathfrak{p} \mathfrak{C}(\phi)} A_{\mathfrak{p}}\left(\vartheta(\eta)^{p \text {-st }} ; \phi, j\right) L\left(\vartheta(\eta)^{p \text {-st }}, \phi^{-1}, j\right) .
\end{aligned}
$$

Local $\varepsilon$-factor at $p$. - Recall that for each $\mathfrak{P}$ in $\Sigma_{p}$ and $\mathfrak{p}=\mathfrak{P P}^{c}$, we obtain a specified identification

$$
\mathfrak{r}_{F^{+}, \mathfrak{p}} \stackrel{\sim}{\rightarrow} \mathfrak{r}_{F, \mathfrak{P}}
$$

induced by $\tau_{\mathfrak{p}}(x) \mapsto \sigma_{\mathfrak{P}}(x)$ for each element $x$ of $\mathfrak{r}_{F^{+}}$, where $\sigma_{\mathfrak{P}}: F \hookrightarrow \overline{\mathbb{Q}}$ denotes a unique embedding contained in $\Sigma$ such that $\iota_{p} \circ \sigma_{\mathfrak{P}}$ induces $\mathfrak{P}$, and $\tau_{\mathfrak{p}}$ denotes the restriction of $\sigma_{\mathfrak{P}}$ to $F^{+}$. Then $(2 \delta)_{\mathfrak{P}}=\sigma_{\mathfrak{P}}(2 \delta)$ corresponds to a unique element $(2 \delta)_{\mathfrak{p}}$ of $\mathfrak{r}_{F^{+}, \mathfrak{p}}^{\times}$under the identification (2.19). We define $\varpi_{\mathfrak{p}}$ as an element of $\mathfrak{r}_{F^{+}, \mathfrak{p}}$ corresponding to the fixed uniformiser $\varpi_{\mathfrak{P}}$ of $F_{\mathfrak{P}}$ via (2.19), which is a uniformiser of $F_{\mathfrak{p}}^{+}$.

Note that the idèlic character corresponding to $\eta^{\text {gal }} \chi_{p, \text { cyc }}^{j} \phi$ is $\eta|\cdot|_{\mathbb{A}_{F}}^{j} \phi^{-1}$, where $|\cdot|_{\mathbb{A}_{F}}$ denotes the idèlic norm character on $F$ (see Example 2.1 for details). Here we use the same 
symbol $\phi^{-1}$ for the idèlic character corresponding to the ideal character $\phi^{-1}$ by abuse of notation. Since $\eta|\cdot|_{\mathbb{A}_{F}}^{j}$ is unramified at each place $\mathfrak{P}$ in $\Sigma_{p}$ by virtue of the assumption $\left(\operatorname{unr}_{F^{+}}\right.$), the exponent $e(\mathfrak{P})$ of $\mathfrak{P} \in \Sigma_{p}$ in the conductor of $\eta|\cdot|_{\mathbb{A}_{F}}^{j} \phi^{-1}$ exactly equals the exponent of $\mathfrak{p}=\mathfrak{P P}^{c}$ in the conductor of $\phi$, which we denote by $e_{\phi}(\mathfrak{p})$. By using these facts, we calculate the local $\varepsilon$-factor $W_{p}\left(\eta|\cdot|_{\mathbb{A}_{F}}^{j} \phi^{-1}\right)$ in the following. For each $\mathfrak{P}$ in $\Sigma_{p}$, set

$$
\begin{aligned}
& W_{\mathfrak{P}}\left(\eta|\cdot|_{\mathbb{A}_{F}}^{j} \phi^{-1}\right)
\end{aligned}
$$

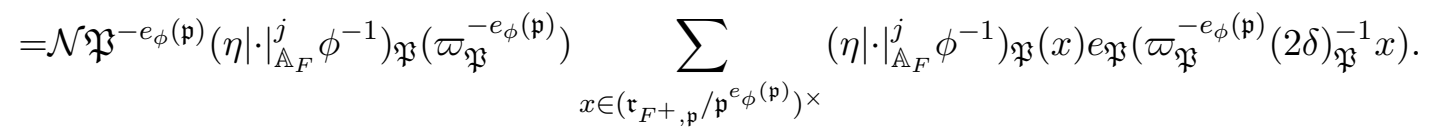

Then $W_{p}\left(\eta|\cdot|_{\mathbb{A}_{F}}^{j} \phi^{-1}\right)$ obviously equals the product $\prod_{\mathfrak{P} \in \Sigma_{p}} W_{\mathfrak{P}}\left(\eta|\cdot|_{\mathbb{A}_{F}}^{j} \phi^{-1}\right)$. Note also that when $e_{\phi}(\mathfrak{p})$ equals 0 , or in other words, when $\phi$ does not ramify at $\mathfrak{p}$, the local term $W_{\mathfrak{P}}\left(\eta|\cdot|_{\mathbb{A}_{F}}^{j} \phi^{-1}\right)$ at $\mathfrak{P}$ is trivial by its definition.

Now assume that $\phi$ is ramified at $\mathfrak{p}=\mathfrak{P P}^{c}$. Then by direct computation, we have

$$
\begin{aligned}
\mathcal{N} \mathfrak{P}^{-e_{\phi}(\mathfrak{p})}\left(\eta|\cdot|_{\mathbb{A}_{F}}^{j} \phi^{-1}\right)_{\mathfrak{P}}\left(\varpi_{\mathfrak{P}}^{-e_{\phi}(\mathfrak{p})}\right)=\left(\frac{\mathcal{N} \mathfrak{P}^{j-1}}{\eta^{*}(\mathfrak{P})}\right)^{e_{\phi}(\mathfrak{p})} \phi_{\mathfrak{p}}^{-1}\left(\mathcal{N}_{F / F^{+}} \varpi_{\mathfrak{P}}^{-e_{\phi}(\mathfrak{p})}\right) \\
=A_{\mathfrak{p}}\left(\vartheta(\eta)^{p-\mathrm{st}}, \phi, j\right) \phi_{\mathfrak{p}}\left(\varpi_{\mathfrak{p}}^{e_{\phi}(\mathfrak{p})}\right) .
\end{aligned}
$$

Next let us take an element $\alpha$ of $F^{+}$satisfying $\alpha_{\mathfrak{p}}=\varpi_{\mathfrak{p}}^{e_{\phi}(\mathfrak{p})}$ for each $\mathfrak{p}$ of $F^{+}$lying above $p$. Note that $\left(\eta|\cdot|_{\mathbb{A}_{F}}^{j}\right)_{\mathfrak{P}}(x)$ is trivial for each $\mathfrak{P}$ in $\Sigma_{p}$ and an arbitrary element $x$ in $\mathfrak{r}_{\mathfrak{P}}^{\times}$by virtue of the assumption $\left(\operatorname{unr}_{F^{+}}\right)$. By using this, we can calculate as

$$
\begin{aligned}
& \phi_{\mathfrak{p}}\left(\varpi_{\mathfrak{p}}^{e_{\phi}(\mathfrak{p})}\right) \sum_{x \in\left(\mathfrak{r}_{F, \mathfrak{P}} / \mathfrak{P}^{e(\mathfrak{P})}\right) \times}\left(\eta|\cdot|_{\mathbb{A}_{F}}^{j} \phi^{-1}\right)_{\mathfrak{P}}(x) e_{\mathfrak{P}}\left(\varpi_{\mathfrak{P}}^{-e(\mathfrak{P})}(2 \delta)_{\mathfrak{P}}^{-1} x\right) \\
& =\phi_{\mathfrak{p}}\left(\varpi_{\mathfrak{p}}^{\left.e_{\phi}^{(\mathfrak{p})}\right)} \sum_{x \in\left(\mathfrak{r}_{F^{+}, \mathfrak{p}} / \mathfrak{p}^{e_{\phi}(\mathfrak{p})}\right)^{\times}} \phi_{\mathfrak{p}}^{-1}(x) e_{\mathfrak{p}}\left(\varpi_{\mathfrak{p}}^{-e_{\phi}(\mathfrak{p})}(2 \delta)_{\mathfrak{p}}^{-1} x\right)\right. \\
& =\phi_{\mathfrak{p}}^{-1}\left((-2 \delta)_{\mathfrak{p}}\right) \sum_{x \in\left(\mathfrak{r}_{F^{+}, \mathfrak{p}} / \mathfrak{p}^{e} \phi^{(\mathfrak{p})}\right)^{\times}} \phi_{\mathfrak{p}}^{-1}\left(x \alpha_{\mathfrak{p}}^{-1}\right) e_{\mathfrak{p}}\left(-x \alpha_{\mathfrak{p}}^{-1}\right)
\end{aligned}
$$

In the third equality we change the variable of the summation using the fact that the correspondance $x \mapsto x(-2 \delta)_{\mathfrak{p}}$ induces an automorphism of $\left(\mathfrak{r}_{F^{+}, \mathfrak{p}} / \mathfrak{p}^{e_{\phi}(\mathfrak{p})}\right)^{\times}$. Since $2 \delta$ is a $p$-adic unit, the evaluation of the $p$-adic avatar of the norm character $|\cdot|_{\mathbb{A}_{F}+}$ at the idèle of $F^{+}$defined as $(2 \delta)_{\mathfrak{p r}_{F^{+}}}=\left((2 \delta)_{\mathfrak{p}}\right)_{\mathfrak{p} \mid p \mathfrak{r}_{F^{+}}}$is calculated as $\prod_{\mathfrak{P} \in \Sigma_{p}} \sigma_{\mathfrak{P}}(2 \delta)^{\sharp \Sigma_{\mathfrak{P}}}$, which coincides with $(-1)^{d} \tilde{d}=(-1)^{d} \chi_{\mathrm{cyc}, p}\left(z_{\tilde{d}}\right)$. This implies that the idèle $(-2 \delta)_{p \mathbf{r}_{F^{+}}}$corresponds to the element $z_{\tilde{d}}$ of $\operatorname{Gal}\left(F^{+}\left(\mu_{p^{\infty}}\right) / F^{+}\right)$via the reciprocity map in global class field theory. In particular, the product $\prod_{\mathfrak{p} \mid p \mathfrak{r}_{F^{+}}} \phi_{\mathfrak{p}}^{-1}\left((-2 \delta)_{\mathfrak{p}}\right)$ coincides with $\phi\left(z_{\tilde{d}}\right)$. Note that, by the definition of the ideal characters corresponding to the größencharacters, the equality $\prod_{\mathfrak{p} \mid p \mathfrak{r}_{F^{+}}} \phi_{\mathfrak{p}}^{-1}\left(\left(x \alpha^{-1}\right)_{\mathfrak{p}}\right)=\phi\left(x \alpha^{-1} \mathfrak{r}_{F^{+}}\right)$holds for an arbitrary element $x$ in $F^{+}$relatively prime to $p$. Taking the product of (2.20) over prime ideals $\mathfrak{p}$ of $\mathfrak{r}_{F^{+}}$lying above $p$, we 
obtain the equality

$$
\begin{aligned}
\prod_{\mathfrak{p} \mid p \mathfrak{r}_{F^{+}}} \phi_{\mathfrak{p}}^{-1}\left((-2 \delta)_{\mathfrak{p}}\right) & \sum_{x \in\left(\mathfrak{r}_{F^{+}, \mathfrak{p}} / \mathfrak{p}^{e} \phi^{(\mathfrak{p})}\right)^{\times}} \phi_{\mathfrak{p}}^{-1}\left(x \alpha_{\mathfrak{p}}^{-1}\right) e_{\mathfrak{p}}\left(-x \alpha_{\mathfrak{p}}^{-1}\right) \\
=\phi\left(z_{\tilde{d}}\right) & \sum_{x \in\left(\mathfrak{r}_{F^{+}} / \mathfrak{C}(\phi)\right)^{\times}} \phi\left(x \alpha^{-1} \mathfrak{r}_{F^{+}}\right) \exp \left(2 \pi \sqrt{-1} \operatorname{Tr}_{F^{+} / \mathbb{Q}}\left(x \alpha^{-1}\right)\right),
\end{aligned}
$$

and the last term is no other than the product of $\phi\left(z_{\tilde{d}}\right)$ and the Gaussian sum $G(\phi)$ defined as (2.13) under the isomorphism

$$
\left(\mathfrak{C}(\phi)^{-1} / \mathfrak{r}_{F^{+}}\right)^{\times} \stackrel{\sim}{\rightarrow}\left(\mathfrak{r}_{F^{+}} / \mathfrak{C}(\phi)\right)^{\times} ; x \mapsto \alpha x .
$$

Consequently we obtain

$$
W_{p}\left(\eta|\cdot|_{\mathbb{A}_{F}}^{j} \phi^{-1}\right)=\prod_{\mathfrak{P} \in \Sigma_{p}} W_{\mathfrak{P}}\left(\eta|\cdot|_{\mathbb{A}_{F}}^{j} \phi^{-1}\right)=\phi\left(z_{\tilde{d}}\right) G(\phi) \prod_{\mathfrak{p}\left|p \mathfrak{r}_{F}, \mathfrak{p}\right| \mathfrak{C}(\phi)} A_{\mathfrak{p}}\left(\vartheta(\eta)^{p-\mathrm{st}}, \phi, j\right) .
$$

- Other coefficients. - One calculates as

$$
\begin{aligned}
\left(\mathfrak{r}_{F}^{\times}: \mathfrak{r}_{F^{+}}^{\times}\right) & \frac{(-1)^{w_{j} d}(2 \pi)^{\left|r_{j}\right|} \Gamma_{\Sigma}\left(w_{j} \mathrm{t}+\mathrm{r}_{j}\right)}{\sqrt{\left|D_{F^{+}}\right|} \operatorname{Im}(2 \delta)^{\mathrm{r}_{j}}} \\
= & \left(\mathfrak{r}_{F}^{\times}: \mathfrak{r}_{F^{+}}^{\times}\right) \frac{(-1)^{2 j-\left[\kappa_{\mu}\right]}(-2 \pi \sqrt{-1})^{\left|\kappa_{\mu, 2}-j\right|} \Gamma\left(j \mathrm{t}-\kappa_{\mu, 1}\right)}{\sqrt{\left|D_{F^{+}}\right|}(-2 \delta)^{\kappa_{\mu, 2}-j \mathrm{t}}} \\
& =\frac{\Gamma\left(j \mathrm{t}-\kappa_{\mu, 1}\right)}{(-2 \pi \sqrt{-1})^{j d}} \cdot \frac{(-1)^{\left[\kappa_{\mu}\right]}\left(\mathfrak{r}_{F}^{\times}: \mathfrak{r}_{F^{+}}^{\times}\right)(-2 \pi \sqrt{-1})^{\left|\kappa_{\mu, 2}\right|}(-2 \delta)^{j \mathrm{t}}}{\sqrt{\left|D_{F^{+}}\right|}(-2 \delta)^{\kappa_{\mu, 2}}} .
\end{aligned}
$$

Combining all the equations (2.16), (2.17), (2.18), (2.21) and (2.22), we obtain the desired interpolation formula (2.15) of the $p$-adic measure $\mathcal{L}_{p, \mathrm{CM}}^{\mathrm{cyc}}(\eta)$.

As in Section 2.2.2, we identify the archimedean part of $\mathrm{Cl}_{F^{+}}^{+}\left(p^{\infty} \mathfrak{r}_{F^{+}}\right)$with $\{ \pm 1\}^{I_{F^{+}}}$, and denote by $w_{\tau}$ for each $\tau$ in $I_{F^{+}}$the element of the archimedean part of $\mathrm{Cl}_{F^{+}}^{+}\left(p^{\infty} \mathfrak{r}_{F^{+}}\right)$ such that only its $\tau$-component equals to -1 . Set $e_{\tau}^{ \pm}=\left(1 \pm w_{\tau}\right) / 2$ and define an idempotent $e_{\epsilon}$ of $\mathcal{O}\left[\left[\mathrm{Cl}_{F^{+}}^{+}\left(p^{\infty} \mathfrak{r}_{F^{+}}\right)\right]\right]$by

$$
e_{\epsilon}=\prod_{\tau \in I_{F^{+}}} e_{\tau}^{\epsilon_{\tau}}
$$

for each $\epsilon=\left(\epsilon_{\tau}\right)_{\tau \in I_{F^{+}}}$in $\{ \pm 1\}^{I_{F^{+}}}$; then for a ray class character $\phi: \mathrm{Cl}_{F^{+}}^{+}\left(p^{\infty} \mathfrak{r}_{F^{+}}\right) \rightarrow \mathbb{C}^{\times}$ of finite order, we have

$$
\phi\left(e_{\epsilon}\right)= \begin{cases}1 & \text { when } \epsilon \text { coincides with } \operatorname{sgn}(\phi), \\ 0 & \text { otherwise }\end{cases}
$$

By abuse of notation, we also use the same notation $e_{\epsilon}$ for the image of $e_{\epsilon}$ under the composite map

$$
\left.\mathcal{O}\left[\left[\mathrm{Cl}_{F^{+}}^{+}\left(p^{\infty} \mathfrak{r}_{F^{+}}\right)\right]\right] \stackrel{\text { rec }}{\longrightarrow} \mathcal{O}\left[\left[\operatorname{Gal}\left(F_{p^{\infty} \mathfrak{r}_{F^{+}}}^{+} / F^{+}\right)\right]\right] \rightarrow \mathcal{O}\left[\left[\operatorname{Gal}\left(F^{+}\left(\mu_{p^{\infty}}\right) / F^{+}\right)\right)\right]\right] .
$$

Then, by comparing the interpolation formulae (2.14) and (2.15), we obtain the following equality holds between the two different $p$-adic $L$-functions $\mathcal{L}_{p, \mathrm{CM}}^{\text {cyc }}(\eta)$ and $\mathcal{L}_{p}^{\text {cyc }}(\vartheta(\eta))$ in 


$$
\begin{aligned}
\widehat{\mathcal{O}}^{\mathrm{ur}}\left[\left[\operatorname{Gal}\left(F^{+}\left(\mu_{p \infty}\right) / F^{+}\right)\right]\right]: & \\
\mathcal{L}_{p, \mathrm{CM}}^{\mathrm{cyc}}(\eta) & =\sum_{\epsilon \in\{ \pm 1\}^{I} F^{+}} \frac{\Gamma\left(\left(\kappa_{\mu, 1}^{\max }+1\right) \mathrm{t}-\kappa_{\mu, 1}\right) C_{\vartheta(\eta), \infty}^{\epsilon}}{\left(-\widetilde{\Omega}_{\mathrm{CM}, \infty}\right)^{\kappa_{\mu, 2}}\left(-\Omega_{\mathrm{CM}, \infty}\right)^{-\kappa_{\mu, 1}}} \mathcal{L}_{p}^{\mathrm{cyc}}(\vartheta(\eta)) .
\end{aligned}
$$

In particular we obtain the main result of the analytic part:

Corollary 2.25 (Theorem $\mathrm{A}$ ). The p-adic measure $\mathcal{L}_{p, \mathrm{CM}}^{\mathrm{cyc}}(\eta)$ is a nonzero constant multiple of the cyclotomic p-adic zeta function $\mathcal{L}_{p}^{\text {cyc }}(\vartheta(\eta))$ associated to $\vartheta(\eta)$ in each component of the semilocal Iwasawa algebra $\widehat{\mathcal{O}}^{\mathrm{ur}}\left[\left[\operatorname{Gal}\left(F^{+}\left(\mu_{p^{\infty}}\right) / F^{+}\right)\right]\right]$, and each of the two p-adic Lfunctions generates the same ideal in $\widehat{\mathcal{O}}^{\mathrm{ur}}\left[\left[\mathrm{Gal}\left(F^{+}\left(\mu_{p^{\infty}}\right) / F^{+}\right)\right]\right] \otimes_{\mathbb{Z}_{p}} \mathbb{Q}_{p}$.

It is widely believed that Corollary 2.25 holds even in $\widehat{\mathcal{O}}^{\text {ur }}\left[\left[\operatorname{Gal}\left(F^{+}\left(\mu_{p^{\infty}}\right) / F^{+}\right)\right]\right]$(or in other words, $\mathcal{L}_{p, \mathrm{CM}}^{\mathrm{cyc}}(\eta)$ and $\mathcal{L}_{p}^{\text {cyc }}(\vartheta(\eta))$ have "the same $\mu$-invariants"), and this speculation leads us to make a conjecture on the ratio of two complex periods constructed in completely different manners.

Conjecture 2.26. The ratio of the complex periods

$$
\frac{\Gamma\left(\left(\kappa_{\mu, 1}^{\max }+1\right) \mathrm{t}-\kappa_{\mu, 1}\right) C_{\vartheta(\eta), \infty}^{\epsilon}}{\left(-\widetilde{\Omega}_{\mathrm{CM}, \infty}\right)^{\kappa_{\mu, 2}}\left(-\Omega_{\mathrm{CM}, \infty}\right)^{-\kappa_{\mu, 1}}}
$$

is a $p$-adic unit for an arbitrary element $\epsilon$ in $\{ \pm 1\}^{I{ }^{+}}$with respect to the fixed embedding $\iota_{p}: \overline{\mathbb{Q}} \hookrightarrow \overline{\mathbb{Q}}_{p}$.

Remark 2.27. In the case where $F^{+}$is the rational number field $\mathbb{Q}$ (elliptic modular cases), the second-named author and Prasanna have obtained a partial result to this conjecture (see [OP, Theorem 6.1]) using the nonvanishing modulo $p$ of special values of the $L$-functions associated to elliptic cuspform (due to Stevens [St85, Theorem 2.1] and Ash-Stevens [AS86]) and the modular parametrisation of an elliptic curve with complex multiplication. However,the nonvanishing modulo $p$ of special values of the $L$-functions has not been generalised to general Hilbert modular cuspforms yet, and there seems to be no generalisation of the theory of modular parametrisation to Hilbert-Blumenthal modular varieties. Hence it seems difficult to generalise the proof of [OP, Theorem 6.1] to general Hilbert modular cases at the present.

\section{The ALGEBRAIC SIDE}

We establish algebraic parts of our main results and apply them to the Iwasawa main conjecture for Hilbert modular cuspforms with complex multiplication in this section. We first introduce the Selmer groups associated to nearly $p$-ordinary Hilbert cuspforms with complex multiplication, and compare them with the Iwasawa module obtained as a certain Galois group $\operatorname{Gal}\left(M_{\Sigma_{p}} / \widetilde{K}_{\infty}^{\mathrm{CM}}\right)$ (Section 3.1). Then we verify the (exact) control theorem (Theorem 3.18) which describes the behaviour of the (multi-variable) Selmer groups under specialisation procedures (Section 3.2). We finally discuss the almost divisibility of the strict Selmer groups and basechange compatibility of the characteristic ideals of their Pontrjagin duals (Sections 3.3 and 3.4). As an application, we discuss the validity of the cyclotomic Iwasawa main conjecture for Hilbert modular cuspforms with complex multiplication (Section 3.5). 
3.1. Selmer groups. This subsection is devoted to the definition of various Selmer groups and the comparison among them. In Section 3.1.2 we first recall the general definition of Selmer groups $\operatorname{Sel}_{\mathcal{A}}$ associated to deformations of Galois representations after Greenberg (Definition 3.1), and then define the Selmer group $\operatorname{Sel}_{\mathcal{A}_{f}^{\text {cyc }}}$ by applying this general recipe to the cyclotomic deformation $\mathcal{A}_{f}^{\text {cyc }}$ of the Galois representation $A_{f}^{\text {cyc }}$ associated to a nearly $p$-ordinary Hilbert eigencuspform $f$. When the Hilbert cuspform $f$ has complex multiplication, or in other words, when $f$ is represented as (the $p$-stabilisation of) the theta lift $\vartheta(\eta)$ of a größencharacter $\eta$ of type $\left(A_{0}\right)$ defined on a certain totally imaginary quadratic extension $F$ of $F^{+}$, we identify $\operatorname{Sel}_{\mathcal{A}_{f}^{\text {cyc }}}$ with the Selmer group $\operatorname{Sel}_{\mathcal{A}_{\eta}^{\text {cyc }}}^{\Sigma}$ associated to the cyclotomic deformation of $\eta$ defined with respect to a fixed CM type $\Sigma$ of $F$ (see Lemma 3.5). In Section 3.1.4, we introduce the (multi-variable) Selmer group $\operatorname{Sel}_{\mathcal{A}_{\eta}^{\text {CM }}}^{\Sigma}$ associated to the deformation of $\eta$ along the field extension $\widetilde{F}_{\infty} / F$, and prove in Proposition 3.16 that the characteristic ideal of the Pontrjagin dual of $\operatorname{Sel}_{\mathcal{A}_{\eta}^{\text {CM }}}^{\Sigma}$ coincides with (a certain twist of) the characteristic ideal of the $\psi$-isotypic quotient $X_{\Sigma_{p},(\psi)}$ of the Iwasawa module $X_{\Sigma_{p}}$ defined in a classical way (here $\psi$ denotes a branch character associated to $\eta$; see Lemma 3.7 for details on the branch character $\psi$ ).

3.1.1. General definition. We first recall the general notion of Selmer groups for deformations of Galois representations, which is introduced by Ralph Greenberg Gr94, Sections 3 and 4].

Let $\mathcal{R}$ be a complete, noetherian semilocal ring of characteristic 0 , and suppose that the residue field $\mathcal{R} / \mathfrak{M}$ of $\mathcal{R}$ is a finite field of characteristic $p$ for each maximal ideal $\mathfrak{M}$ of $\mathcal{R}$. Let $\mathrm{K}$ be a number field and $\mathcal{T}$ a free $\mathcal{R}$-module of finite rank on which the absolute Galois group $G_{\mathrm{K}}$ of $\mathrm{K}$ acts continuously and $\mathcal{R}$-linearly. We impose the following constraint on the $\mathcal{R}$-linear $G_{\mathrm{K}}$-representation $\mathcal{T}$ :

the Galois action on $\mathcal{T}$ is unramified outside a finite set $S$ of places of $\mathrm{K}$ which contains all the places lying above $p$ and all the archimedean places.

Then the action of $G_{\mathrm{K}}$ on $\mathcal{T}$ factors through the Galois group $\mathrm{Gal}\left(\mathrm{K}_{S} / \mathrm{K}\right)$ of the maximal Galois extension $\mathrm{K}_{S}$ over $\mathrm{K}$ which is unramified outside the places of $S$. For each prime ideal $\mathfrak{p}$ of $\mathrm{K}$ lying above $p$, we specify an $\mathcal{R}$-direct summand $\mathrm{Fil}_{\mathfrak{p}}^{+} \mathcal{T}$ of $\mathcal{T}$ which is stable under the action of the decomposition group $D_{\mathfrak{p}}$ of $G_{\mathfrak{K}}$ at $\mathfrak{p}$. In many cases, there exists a canonical (and unique) choice of such a direct summand $\mathrm{Fil}_{\mathfrak{p}}^{+} \mathcal{T}$ for each $\mathfrak{p}$. If the Galois representation $\mathcal{T}$ is ordinary (or nearly ordinary) at each place $\mathfrak{p}$ above $p$, for example, there exists a canonical direct summand $\mathrm{Fil}_{\mathfrak{p}}^{+} \mathcal{T}$ of $\mathcal{T}$ induced from what is called the ordinary filtration at each $\mathfrak{p}$. In Section 3.3.2 we shall introduce more general notion of local conditions concerning the definition of Selmer groups. We denote the Pontrjagin dual $\operatorname{Hom}_{\text {cts }}\left(\mathcal{R}, \mathbb{Q}_{p} / \mathbb{Z}_{p}\right)$ of $\mathcal{R}$ by $\mathcal{R}^{\vee}$. Now consider the discrete $\mathcal{R}$-module $\mathcal{A}$ defined as $\mathcal{A}=\mathcal{T} \otimes_{\mathcal{R}} \mathcal{R}^{\vee}$. The absolute Galois group $G_{\mathrm{K}}$ acts on $\mathcal{A}$ via the first factor and we regard $\mathcal{A}$ as a discrete $\mathcal{R}$-linear Galois representation of $\operatorname{Gal}\left(\mathrm{K}_{S} / \mathrm{K}\right)$. Note that $\mathcal{A}$ is equipped with the specified direct summand $\mathrm{Fil}_{\mathfrak{p}}^{+} \mathcal{A}$ at each place $\mathfrak{p}$ above $p$ which is induced from the specification of direct summands of $\mathcal{T}$; namely, $\mathrm{Fil}_{\mathfrak{p}}^{+} \mathcal{A}$ is defined as $\mathrm{Fil}_{\mathfrak{p}}^{+} \mathcal{T} \otimes_{\mathcal{R}} \mathcal{R}^{\vee}$.

Definition 3.1 (Greenberg's Selmer group). The (Greenberg's) Selmer group $\operatorname{Sel}_{\mathcal{A}}$ associated to $\mathcal{A}$ is defined as the kernel of the global-to-local morphism

$$
H^{1}\left(\mathrm{~K}_{S} / \mathrm{K}, \mathcal{A}\right) \longrightarrow \prod_{\substack{\lambda \in S \\ \lambda \nmid p \infty}} H^{1}\left(I_{\lambda}, \mathcal{A}\right) \times \prod_{\substack{\mathfrak{p} \in S \\ \mathfrak{p} \mid p \mathfrak{K}^{-}}} H^{1}\left(I_{\mathfrak{p}}, \mathcal{A} / \mathrm{Fil}_{\mathfrak{p}}^{+} \mathcal{A}\right)
$$


induced by the restriction maps of Galois cohomology groups. Here $I_{v}$ denotes the inertia subgroup of the absolute Galois group $G_{\mathrm{K}}$ at each finite place $v$ of $\mathrm{K}$.

3.1.2. Selmer groups for cyclotomic deformations. Let us recall the following theorem which is due to many people including Ohta Oh83], Carayol [Ca86], Wiles [Wi88], Taylor Tay89 and Blasius and Rogawski [BlRo94]. We quote [Wi88, Theorem 1,2] for nearly ordinary situations as follows:

Theorem 3.2. Let $f$ be a nearly p-ordinary normalised eigencuspform defined on a totally real number field $F^{+}$satisfying (unr), which is of cohomological weight $\kappa$, level $\mathfrak{N}$ and nebentypus $\underline{\varepsilon}$. Let $\mathcal{K}$ be a finite extension of $\mathbb{Q}_{p}$ containing the Hecke field $\mathbb{Q}_{f}$ of $f$ and $\mathcal{O}$ the ring of integers of $\mathcal{K}$.

Then there exists a 2-dimensional Galois representation $V_{f}$ of $G_{F^{+}}$with coefficients in $\mathcal{K}$ with the following properties:

(1) For every prime ideal $\mathfrak{q}$ which does not divide $\mathfrak{p} \mathfrak{N}$, the following equation holds.

$$
\operatorname{det}\left(1-\operatorname{Frob}_{\mathfrak{q}} X ; V_{f}\right)=1-C(\mathfrak{q} ; f) X+\mathcal{N} \mathfrak{q} \varepsilon_{+}(\mathfrak{q}) X^{2}
$$

(2) For each place $\mathfrak{p}$ of $F^{+}$lying above $p$, we have a $D_{\mathfrak{p}}$-stable $\mathcal{K}$-subspace Fil $_{\mathfrak{p}}^{+} V_{f} \subset V_{f}$ of dimension one on which the action of $D_{\mathfrak{p}}$ is unramified.

The Galois representation $V_{f}$ of $G_{F^{+}}$is called the Galois representation associated to $f$.

We define $S^{+}$as a finite set of places of $F^{+}$consisting of all the archimedean places and all the finite places dividing $p \mathfrak{N}$. Then the action of $G_{F^{+}}$on $V_{f}$ factors through the quotient $\operatorname{Gal}\left(F_{S^{+}}^{+} / F^{+}\right)$of $G_{F^{+}}$. Let $\Lambda_{\mathcal{O}}^{\text {cyc }}$ denote the Iwasawa algebra $\mathcal{O}\left[\left[\operatorname{Gal}\left(F^{+}\left(\mu_{p^{\infty}}\right) / F^{+}\right)\right]\right]$over $\mathcal{O}$. Note that $\Lambda_{\mathcal{O}}^{\text {cyc }}$ satisfies all the conditions which we have imposed on the coefficient ring

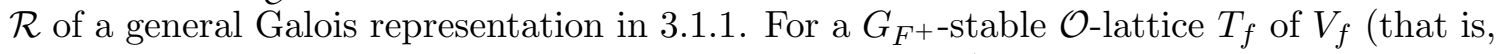
a $G_{F^{+}-\text {stable }} \mathcal{O}$-submodule of $V_{f}$ satisfying $T_{f} \otimes_{\mathcal{O}} \mathcal{K}=V_{f}$ ), set

$$
\mathcal{T}_{f}^{\text {cyc }}=T_{f} \otimes_{\mathcal{O}} \Lambda_{\mathcal{O}}^{\text {cyc, } \sharp}
$$

and let $G_{F^{+}}$act on $\mathcal{T}_{f}^{\text {cyc }}$ diagonally (refer to Notation in Introduction on the superscript $\sharp)$. The $G_{F^{+}}$-module $\mathcal{T}_{f}^{\text {cyc }}$ is called the cyclotomic deformation of $T_{f}$. For each place $\mathfrak{p}$ of $F^{+}$lying above $p$, we define $\mathrm{Fil}_{\mathfrak{p}}^{+} \mathcal{T}_{f}^{\text {cyc }}$ as

$$
\mathrm{Fil}_{\mathfrak{p}}^{+} \mathcal{T}_{f}^{\mathrm{cyc}}=\mathrm{Fil}_{\mathfrak{p}}^{+} T_{f} \otimes_{\mathcal{O}} \Lambda_{\mathcal{O}}^{\mathrm{cyc}, \sharp}
$$

equipped with the diagonal action of $G_{F^{+}}$(here we define $\mathrm{Fil}_{\mathfrak{p}}^{+} T_{f}$ as the intersection of $T_{f}$ and $\mathrm{Fil}_{\mathfrak{p}}^{+} V_{f}$ ).

Definition 3.3 (Selmer group $\mathrm{Sel}_{\mathcal{A}_{f}^{\text {cyc }}}$ ). The Selmer group $\mathrm{Sel}_{\mathcal{A}_{f}^{\text {cyc }}}$ associated to the cyclotomic deformation of $f$ is the Selmer group defined as in Definition 3.1 for the discrete $\Lambda_{\mathcal{O}}^{\text {cyc }}$-linear $G_{F^{+}}$-representation $\mathcal{A}_{f}^{\text {cyc }}=\mathcal{T}_{f}^{\text {cyc }} \otimes_{\Lambda_{\mathcal{O}}^{\text {cyc }}} \Lambda_{\mathcal{O}}^{\text {cyc }, \vee}$.

Note that $\operatorname{Sel}_{\mathcal{A}_{f}^{\text {cyc }}}$ does depend on the choice of $G_{F^{+}}$-stable $\mathcal{O}$-lattices $T_{f}$. When $V_{f}$ is

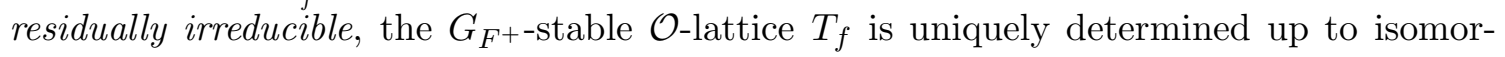
phisms, and hence the Selmer group $\operatorname{Sel}_{\mathcal{A}_{f}^{\text {cyc }}}$ is also uniquely determined up to isomorphisms (independently of the choice of $T_{f}$ ). 
3.1.3. Cyclotomic deformations of Hilbert modular cuspforms with complex multiplication. From now on let us assume that $f$ is a nearly p-ordinary p-stabilised newform with complex multiplication. Then by definition there exist a totally imaginary quadratic extension $F$ of $F^{+}$satisfying the ordinarity condition $\left(\operatorname{ord}_{F / F^{+}}\right)$and a größencharacter $\eta$ of type $\left(A_{0}\right)$ on $F$ such that $f$ is represented as the $p$-stabilisation $\vartheta(\eta)^{p \text {-st }}$ of the theta lift $\vartheta(\eta)$ of $\eta$ (see Proposition 2.13). Since Hecke eigenvalues of $\vartheta(\eta)^{p \text {-st }}$ coincide with those of $\vartheta(\eta)$ away from prime ideals lying above $p$, the Galois representation associated to $\vartheta(\eta)^{p \text {-st }}$ is isomorphic to the Galois representation $V_{\vartheta(\eta)}$ associated to $\vartheta(\eta)$ by virtue of Cebotarev's density theorem. We shall recall in Appendix $\AA$ that the Galois representation $V_{\vartheta(\eta)}$ associated to $\vartheta(\eta)$ is isomorphic to the induced representation $\operatorname{Ind}_{F}^{F^{+}} \mathcal{K}\left(\eta^{\text {gal }}\right)$ of the one-dimensional $G_{F^{-}}$ representation $\mathcal{K}\left(\eta^{\text {gal }}\right)$, and hence there is a canonical $G_{F^{+}}$stable $\mathcal{O}$-lattice of $V_{\vartheta(\eta)}$ : namely $\operatorname{Ind}_{F}^{F^{+}} \mathcal{O}\left(\eta^{\text {gal }}\right)$. We thus adopt $\operatorname{Ind}_{F}^{F^{+}} \mathcal{O}\left(\eta^{\text {gal }}\right)$ as the lattice $T_{\vartheta(\eta)}$ used in the construction of the Selmer group. The ordinary filtration $\mathrm{Fil}_{\mathfrak{p}}^{+} T_{\vartheta(\eta)}$ at $\mathfrak{p}$ above $p$ is then identified with $\mathcal{O}\left(\left.\eta^{\text {gal }}\right|_{D_{\mathfrak{P}}}\right)$ where $\mathfrak{P}$ is a unique element of $\Sigma_{p}$ which lies above $\mathfrak{p}$.

We here introduce another Selmer group $\operatorname{Sel}_{\mathcal{A}_{\eta}^{\text {cyc }}}^{\Sigma}$ associated to a größencharacter $\eta$ of the CM number field $F$. The restriction of the action of $\operatorname{Gal}\left(F\left(\mu_{p^{\infty}}\right) / F\right)$ on $F\left(\mu_{p^{\infty}}\right)$ to $F^{+}\left(\mu_{p^{\infty}}\right)$ induces an isomorphism between $\operatorname{Gal}\left(F\left(\mu_{p^{\infty}}\right) / F\right)$ and $\operatorname{Gal}\left(F^{+}\left(\mu_{p^{\infty}}\right) / F^{+}\right)$, which enables us to identify the Iwasawa algebra $\mathcal{O}\left[\left[\operatorname{Gal}\left(F\left(\mu_{p^{\infty}}\right) / F\right)\right]\right]$ of $\operatorname{Gal}\left(F\left(\mu_{p \infty}\right) / F\right)$ with $\Lambda_{\mathcal{O}}^{\text {cyc }}:=$ $\mathcal{O}\left[\left[\operatorname{Gal}\left(F^{+}\left(\mu_{p^{\infty}}\right) / F^{+}\right)\right]\right]$in a canonical manner. Now consider the cyclotomic deformation of $\eta^{\text {gal }}$

$$
\mathcal{T}_{\eta}^{\text {cyc }}=\mathcal{O}\left(\eta^{\text {gal }}\right) \otimes_{\mathcal{O}} \Lambda_{\mathcal{O}}^{\text {cyc,\# }}
$$

equipped with the $D_{\mathcal{P}}$-stable filtration $\mathrm{Fil}_{\mathcal{P}}^{+} \mathcal{T}_{\eta}^{\text {cyc }}$ for each prime ideal $\mathcal{P}$ of $F$ lying above $p$ defined by

$$
\mathrm{Fil}_{\mathcal{P}}^{+} \mathcal{T}_{\eta}^{\mathrm{cyc}}= \begin{cases}\mathcal{T}_{\eta}^{\mathrm{cyc}} & \text { if } \mathcal{P} \text { is contained in } \Sigma_{p}, \\ 0 & \text { otherwise }\end{cases}
$$

As usual we let $G_{F}$ act diagonally on $\mathcal{T}_{\eta}^{\text {cyc }}$. Let $S$ denote the set of places of $F$ lying above those of $F^{+}$in $S^{+}$. Then one readily observes that the diagonal action of $G_{F}$ on $\mathcal{T}_{\eta}^{\text {cyc }}$ factors through the quotient $\operatorname{Gal}\left(F_{S} / F\right)$ of $G_{F}$.

Definition 3.4 (Selmer group $\mathrm{Sel}_{\mathcal{A}_{\eta}^{\text {cyc }}}^{\Sigma}$ ). The Selmer group $\mathrm{Sel}_{\mathcal{A}_{\eta}^{\text {cyc }}}^{\Sigma}$ associated to the cyclotomic deformation $\mathcal{A}_{\eta}^{\text {cyc }}$ of $\eta^{\text {gal }}$ (with respect to the $p$-ordinary CM type $\Sigma$ ) is the Selmer group in the sense of Definition 3.1 constructed for the discrete $G_{F}$-representation $\mathcal{A}_{\eta}^{\text {cyc }}$ defined as $\mathcal{T}_{\eta}^{\text {cyc }} \otimes_{\Lambda_{\mathcal{O}}^{\text {cyc }}} \Lambda_{\mathcal{O}}^{\text {cyc, },}$.

Then one easily sees that the Selmer groups defined in Definitions 3.3 and 3.4 coincide; namely,

Lemma 3.5. The Selmer group $\operatorname{Sel}_{\mathcal{A}_{\vartheta(\eta)}^{\text {cyc }}}$ associated to the cyclotomic deformation $\mathcal{A}_{\eta}^{\mathrm{cyc}}$ of $\vartheta(\eta)$ is isomorphic to the Selmer group $\operatorname{Sel}_{\mathcal{A}_{\eta}^{\text {cyc }}}^{\Sigma}$ as a $\Lambda_{\mathcal{O}}^{\text {cyc }}$-module.

Proof. This is a direct consequence of Shapiro's Lemma. Indeed we may identify $\mathcal{A}_{\vartheta(\eta)}^{\text {cyc }}$ with the induced representation $\operatorname{Ind}_{F}^{F^{+}} \mathcal{A}_{\eta}^{\text {cyc }}$ of $\mathcal{A}_{\eta}^{\text {cyc }}$ by construction (under the canonical identification $\left.\mathcal{O}\left[\left[\operatorname{Gal}\left(F\left(\mu_{p^{\infty}}\right) / F\right)\right]\right] \stackrel{\sim}{\rightarrow} \Lambda_{\mathcal{O}}^{\text {cyc }}:=\mathcal{O}\left[\left[\operatorname{Gal}\left(F^{+}\left(\mu_{p^{\infty}}\right) / F^{+}\right)\right]\right]\right)$, and we therefore obtain the following isomorphisms by virtue of (generalised) Shapiro's lemma (see Ru00, 
Proposition B.2] for details):

$$
\begin{aligned}
H^{1}\left(F_{S^{+}}^{+} / F^{+}, \mathcal{A}_{\vartheta(\eta)}^{\text {cyc }}\right) & \cong H^{1}\left(F_{S} / F, \mathcal{A}_{\eta}^{\text {cyc }}\right), \\
H^{1}\left(I_{\mathfrak{l}}, \mathcal{A}_{\vartheta(\eta)}^{\text {cyc }}\right) & \cong \prod_{\mathfrak{L} \mid \mathfrak{l}} H^{1}\left(I_{\mathfrak{L}}, \mathcal{A}_{\eta}^{\text {cyc }}\right)^{\left[\mathbb{F}_{\mathfrak{L}}: \mathbb{F}_{\mathfrak{l}}\right]} \text { for every } \mathfrak{l} \text { in } S \text { which does not divide } p \infty
\end{aligned}
$$

where $\mathbb{F}_{\mathfrak{L}}$ and $\mathbb{F}_{\mathfrak{l}}$ denote the residue fields $\mathfrak{r}_{F} / \mathfrak{L}$ and $\mathfrak{r}_{F^{+}} / \mathfrak{l}$ respectively. Moreover, at each place $\mathfrak{p}$ of $F^{+}$lying above $p$, we obtain the equality:

$$
H^{1}\left(I_{\mathfrak{p}}, \mathcal{A}_{\vartheta(\eta)}^{\mathrm{cyc}} / \mathrm{Fil}^{+} \mathcal{A}_{\vartheta(\eta)}^{\mathrm{cyc}}\right)=H^{1}\left(I_{\mathfrak{p}}, \mathcal{O}\left(\left.\eta^{\text {gal }}\right|_{D_{\mathfrak{P}}}\right) \otimes_{\mathcal{O}} \Lambda_{\mathcal{O}}^{\text {cyc, } \sharp} \otimes_{\Lambda_{\mathcal{O}}} \Lambda_{\mathcal{O}}^{\text {cyc, } \vee}\right)=H^{1}\left(I_{\mathfrak{P}^{c}}, \mathcal{A}_{\eta}^{\mathrm{cyc}}\right),
$$

where $\mathfrak{p r}_{F}=\mathfrak{P P}^{c}$ with $\mathfrak{P}$ in $\Sigma_{p}$ and $\mathfrak{P}^{c}$ in $\Sigma_{p}^{c}$. Hence $\operatorname{Sel}_{\mathcal{A}_{\vartheta(\eta)}^{\text {cyc }}}$ is canonically identified with the kernel of the global-to-local map

$$
H^{1}\left(F_{S} / F, \mathcal{A}_{\eta}^{\mathrm{cyc}}\right) \rightarrow \prod_{w \in S \backslash \Sigma_{p}} H^{1}\left(I_{w}, \mathcal{A}_{\eta}^{\mathrm{cyc}}\right)
$$

which is none other than the Selmer group $\operatorname{Sel}_{\mathcal{A}_{\eta}^{\text {cyc }}}^{\Sigma}$ by Definition 3.4 .

3.1.4. Selmer groups associated to CM fields and Iwasawa modules of classical type. Recall $\widetilde{F}_{\infty}=\widetilde{F}\left(\mu_{p}\right)$ introduced at basic notations given before Theorem A of Introduction. We first define the multi-variable Selmer group $\operatorname{Sel}_{\mathcal{A}_{\eta}^{\mathrm{CM}}}^{\Sigma}$ over $\Lambda_{\mathcal{O}}^{\mathrm{CM}}:=\mathcal{O}\left[\left[\operatorname{Gal}\left(\widetilde{F}_{\infty} / F\right)\right]\right]$ similarly as the Selmer group $\operatorname{Sel}_{\mathcal{A}_{\eta}^{\text {cyc }}}^{\Sigma}$ over $\Lambda_{\mathcal{O}}^{\text {cyc }}$. We set $\mathcal{T}_{\eta}^{\text {CM }}=\mathcal{O}\left(\eta^{\text {gal }}\right) \otimes_{\mathcal{O}} \Lambda_{\mathcal{O}}^{\mathrm{CM}, \sharp}$ and equip it with the diagonal $G_{F}$-action.

Definition 3.6 (Selmer group $\operatorname{Sel}_{\mathcal{A}_{\eta}^{\text {CM }}}^{\Sigma}$ ). The Selmer group $\operatorname{Sel}_{\mathcal{A}_{\eta}^{\mathrm{CM}}}^{\Sigma}$ associated to the deformation $\mathcal{A}_{\eta}^{\mathrm{CM}}$ of $\eta^{\text {gal }}$ along the extension $\widetilde{F}_{\infty} / F$ (with respect to the $p$-ordinary CM type $\Sigma$ ) is the Selmer group defined as in Definition 3.1 for the discrete $G_{F}$-representation $\mathcal{A}_{\eta}^{\mathrm{CM}}=\mathcal{T}_{\eta}^{\mathrm{CM}} \otimes_{\Lambda_{\mathcal{O}}} \Lambda_{\mathcal{O}}^{\mathrm{CM}, \vee}$

In the rest of this subsection we relates the Selmer group $\operatorname{Sel}_{\mathcal{A}_{\eta}^{\mathrm{CM}}}^{\Sigma}$ with a certain Iwasawa module $X_{\Sigma_{p},(\psi)}$ defined in a classical manner by means of the notion of a branch character associated to $\eta$.

Lemma 3.7. Let $\eta$ be a größencharacter of type $\left(A_{0}\right)$ on $F$. Then there exists a p-adic Galois character $\psi: G_{F} \rightarrow \overline{\mathbb{Q}}_{p}^{\times}$of finite order such that $\eta^{\text {gal }} \psi^{-1}: G_{F} \rightarrow \overline{\mathbb{Q}}_{p}^{\times}$factors through the quotient $\operatorname{Gal}\left(\widetilde{F}_{\infty} / F\right)$ of $G_{F}$. Furthermore we may choose such a character $\psi$ so that the composite field of $F\left(\mu_{p}\right)$ and $K_{\psi}$ is abelian over $F$ and linearly disjoint from $\widetilde{F}$ over $F$, where $K_{\psi}$ denotes the field corresponding to the kernel of $\psi$.

Proof. Let us construct a character $\psi$ satisfying the desired properties. First note that the continuous character $\eta^{\text {gal }}$ factors through the Galois group $\operatorname{Gal}\left(F_{\mathfrak{C}(\eta) p^{\infty}} / F\right)$ of the ray class field $F_{\mathfrak{C}(\eta) p^{\infty}}$ modulo $\mathfrak{C}(\eta) p^{\infty}$ over $F$ (recall that $\mathfrak{C}(\eta)$ denotes the conductor of $\eta$ ). Let $\Delta^{\prime}$ denote the maximal torsion subgroup of $\operatorname{Gal}\left(F_{\mathfrak{C}(\eta) p^{\infty}} / F\right)$, which is known to be finite. Then the subfield of $F_{\mathfrak{C}(\eta) p^{\infty}}$ corresponding to $\Delta^{\prime}$ coincides with $\widetilde{F}$ by definition, and we obtain the exact sequence of abelian groups

$$
0 \rightarrow \Delta^{\prime} \rightarrow \operatorname{Gal}\left(F_{\mathfrak{C}(\eta) p^{\infty}} / F\right) \rightarrow \operatorname{Gal}(\widetilde{F} / F) \rightarrow 0
$$

This short exact sequence splits since the Galois group $\operatorname{Gal}(\widetilde{F} / F)$ is a free $\mathbb{Z}_{p}$-module of rank $d+1+\delta_{F, p}$ by definition. Now we take an arbitrary section $s: \operatorname{Gal}(\widetilde{F} / F) \rightarrow \operatorname{Gal}\left(F_{\mathfrak{C}(\eta) p^{\infty}} / F\right)$ 
and denotes by $K^{\prime}$ the intermediate field of $F_{\mathfrak{C}(\eta) p^{\infty}} / F$ corresponding to $s(\operatorname{Gal}(\widetilde{F} / F))$. By construction $\operatorname{Gal}\left(K^{\prime} / F\right)$ is isomorphic to $\Delta^{\prime}$. We then define $\psi$ as the composition

$$
\psi: G_{F} \rightarrow \operatorname{Gal}\left(K^{\prime} / F\right) \cong \Delta^{\prime} \stackrel{\left.\eta^{\mathrm{gal}}\right|_{\Delta^{\prime}}}{\longrightarrow} \overline{\mathbb{Q}}_{p}^{\times} .
$$

By construction, it is obvious that $\eta^{\text {gal }} \psi^{-1}: G_{F} \rightarrow \overline{\mathbb{Q}}_{p}^{\times}$factors through $\operatorname{Gal}\left(\widetilde{F}_{\infty} / F\right)$ and that $K_{\psi}$ is contained in $K^{\prime}$.

Since the short exact sequence (3.2) splits, the fields $K^{\prime}$ and $\widetilde{F}$ are linearly disjoint over $F$. Furthermore since $\left[F\left(\mu_{p}\right): F\right]$ is prime to $p$, the Galois group $\operatorname{Gal}\left(F\left(\mu_{p}\right) / F\right)$ should be contained in the torsion part $\operatorname{Gal}\left(K^{\prime} / F\right)$ of $\operatorname{Gal}\left(F_{\mathfrak{C}(\eta) p^{\infty}} / F\right)$, and therefore the field $K^{\prime}$ contains $F\left(\mu_{p}\right)$. The composite field of $F\left(\mu_{p}\right)$ and $K_{\psi}$, which is a subfield of $K^{\prime}$, is thus abelian over $F$ and linearly disjoint from $\widetilde{F}$ over $F$.

Definition 3.8 (branch character). Let $\eta$ be a größencharacter of type $\left(A_{0}\right)$ on $F$. We call a character $\psi: G_{F} \rightarrow \overline{\mathbb{Q}}_{p}^{\times}$of finite order satisfying the assertions proposed in Lemma 3.7 a branch character associated to $\eta$.

We denote by $K$ the composite field of $F\left(\mu_{p}\right)$ and $K_{\psi}$, and by $\widetilde{K}_{\infty}^{\mathrm{CM}}$ the composite field of $K$ and $\widetilde{F}_{\infty}$ as in Introduction. We set $\Delta=\operatorname{Gal}(K / F), \Delta^{\text {cyc }}=\operatorname{Gal}\left(F\left(\mu_{p}\right) / F\right)$ and $\widetilde{\Gamma}=\operatorname{Gal}(\widetilde{F} / F)$. The cardinality of $\Delta$ is then the product of the order of $\psi$ and the extension degree $\left[K: K_{\psi}\right]$ of $K$ over $K_{\psi}$. Note that $\left[K: K_{\psi}\right]$ is relatively prime to $p$ since it divides $p-1$.

Now let us consider the following commutative diagram with exact rows and columns:

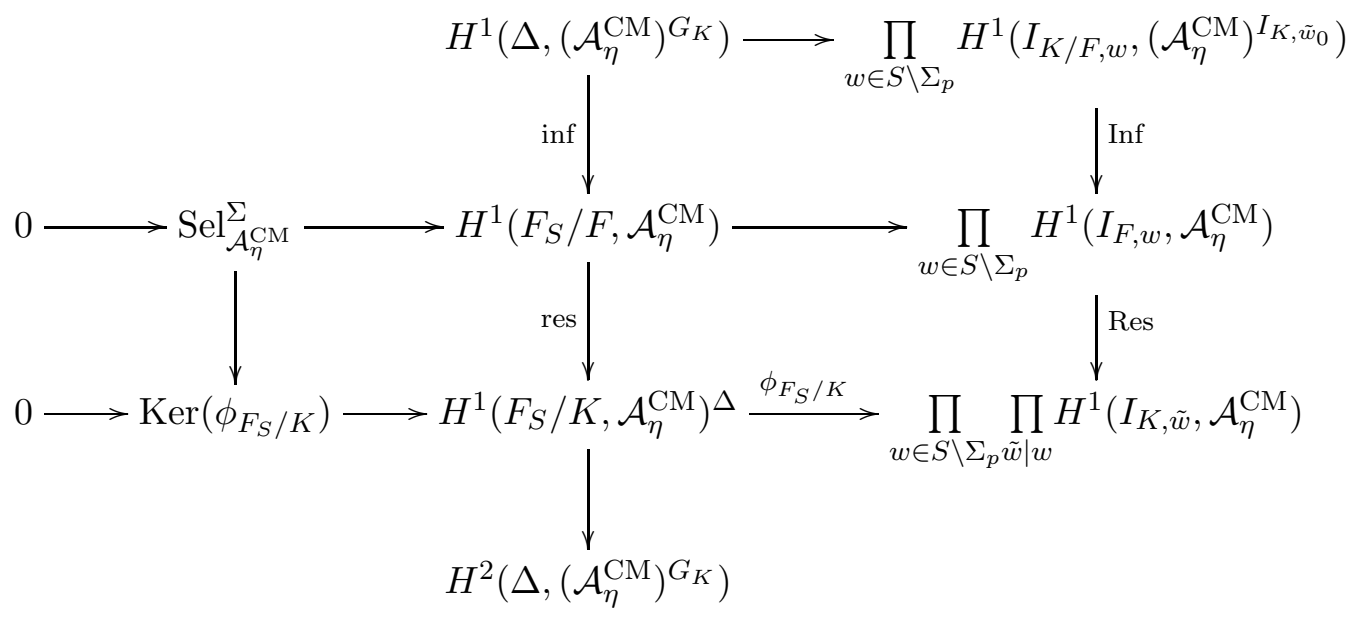

where $\phi_{F_{S} / K}$ denotes the global-to-local map induced from the restriction morphisms. The rows are exact by definition. The middle column of the diagram (3.3) is induced from the inflation-restriction exact sequences associated to the short exact sequence

$$
1 \longrightarrow \operatorname{Gal}\left(F_{S} / K\right) \longrightarrow \operatorname{Gal}\left(F_{S} / F\right) \longrightarrow \Delta \longrightarrow 1
$$

of abelian groups.

The map Res in the right column is induced from restriction maps, and the exactness of the right column is also deduced from the inflation-restriction exact sequence. More precisely, we define the map Res $=(\operatorname{Res})_{w \in S \backslash \Sigma_{p}}$ in the following manner. First we choose and fix a decomposition group $D_{F, w}$ of $\operatorname{Gal}\left(F_{S} / F\right)$ for each place $w$ in $S \backslash \Sigma_{p}$. We always consider the inertia subgroup $I_{F, w}$ of $\operatorname{Gal}\left(F_{S} / F\right)$ to be contained in the fixed decomposition group $D_{F, w}$ at such a place $w$. There exists a unique place $\tilde{w}_{0}$ of $K$ lying above $w$ which is 
fixed under the action of $D_{F, w}$. We identify the intersection of $D_{F, w}$ and $\operatorname{Gal}\left(F_{S} / K\right)$ (resp. the intersection of $I_{F, w}$ and $\operatorname{Gal}\left(F_{S} / K\right)$ ) with the decomposition group $D_{K, \tilde{w}_{0}}$ (resp. the inertia subgroup $\left.I_{K, \tilde{w}_{0}}\right)$ of $\operatorname{Gal}\left(F_{S} / K\right)$ at $\tilde{w}_{0}$. We identify the quotient group $I_{F, w} / I_{K, \tilde{w}_{0}}$ with the inertia subgroup $I_{K / F, w}$ of $\Delta=\operatorname{Gal}(K / F)$ at $w$ (note that the inertia subgroup of $\Delta$ at $w$ is well defined since $\Delta$ is abelian). For each place $\tilde{w}$ of $K$ lying above $w$, we also fix the decomposition group $D_{K, \tilde{w}}$ of $\operatorname{Gal}\left(F_{S} / K\right.$ ) (and denote its inertia subgroup by $I_{K, \tilde{w}}$ ), and choose an element $\sigma_{\tilde{w}}$ of $\operatorname{Gal}\left(F_{S} / F\right)$ so that $\sigma_{\tilde{w}} I_{F, w} \sigma_{\tilde{w}}^{-1}$ contains $I_{K, \tilde{w}}$. Then the map $\operatorname{Res}_{w}$ is defined as the composition

$$
\operatorname{Res}_{w}: H^{1}\left(I_{F, w}, \mathcal{A}_{\eta}^{\mathrm{CM}}\right) \rightarrow \prod_{\tilde{w} \mid w} H^{1}\left(I_{F, w}, \mathcal{A}_{\eta}^{\mathrm{CM}}\right) \stackrel{\sim}{\rightarrow} \prod_{\tilde{w} \mid w} H^{1}\left(\sigma_{\tilde{w}} I_{F, w} \sigma_{\tilde{w}}^{-1}, \mathcal{A}_{\eta}^{\mathrm{CM}}\right) \rightarrow \prod_{\tilde{w} \mid w} H^{1}\left(I_{K, \tilde{w}}, \mathcal{A}_{\eta}^{\mathrm{CM}}\right),
$$

where the first map is the diagonal map, the second one is an isomorphism induced by the conjugation with respect to $\left(\sigma_{\tilde{w}}\right)_{\tilde{w} \mid w}$, and the last map is the usual (componentwise) restriction map. It is easy to observe that the kernel of Res ${ }_{w}$ coincides with the kernel of the restriction map $H^{1}\left(I_{F, w}, \mathcal{A}_{\eta}^{\mathrm{CM}}\right) \rightarrow H^{1}\left(I_{K, \tilde{w}_{0}}, \mathcal{A}_{\eta}^{\mathrm{CM}}\right)$, and hence the right column of the diagram (3.3) is also exact.

Under these settings we shall compare the Selmer group $\operatorname{Sel}_{\mathcal{A}_{\eta}^{\text {cyc }}}$ with $\operatorname{Ker}\left(\phi_{F_{S} / K}\right)$.

Remark 3.9. Since all the cohomology groups $H^{1}\left(\Delta,\left(\mathcal{A}_{\eta}^{\mathrm{CM}}\right)^{G_{K}}\right), H^{2}\left(\Delta,\left(\mathcal{A}_{\eta}^{\mathrm{CM}}\right)^{G_{K}}\right)$ and $H^{1}\left(I_{K / F, w},\left(\mathcal{A}_{\eta}^{\mathrm{CM}}\right)^{I_{K, \tilde{w}_{0}}}\right)$ are $p$-torsion modules annihilated by the cardinality of $\Delta$, all of them vanish when the order of $\psi$ is relatively prime to $p$ (recall that $\left[K: K_{\psi}\right]$ is not divisible by $p$ ). In this case one may immediately conclude that $\operatorname{Sel}_{\mathcal{A}_{\eta}^{\text {CM }}}^{\Sigma}$ is isomorphic to $\operatorname{Ker}\left(\phi_{F_{S} / K}\right)$ by applying the snake lemma to (3.3).

Lemma 3.10. The module $\left(\mathcal{A}_{\eta}^{\mathrm{CM}}\right)^{G_{K}}$ is a copseudonull $\Lambda_{\mathcal{O}}^{\mathrm{CM}}$-module; that is, its Pontrjagin dual is a pseudonull $\Lambda_{\mathcal{O}}^{\mathrm{CM}}$-module. In particular, the cohomology group $H^{i}\left(\Delta,\left(\mathcal{A}_{\eta}^{\mathrm{CM}}\right)^{G_{K}}\right)$ is copseudonull as a $\Lambda_{\mathcal{O}}^{\mathrm{CM}}$-module for $i=1,2$.

Proof. Since $G_{K}$ is a subgroup of $G_{F}$ of finite index, its image $\mathfrak{G}$ under the natural surjection $G_{F} \rightarrow \operatorname{Gal}\left(\widetilde{F}_{\infty} / F\right)$ is also a subgroup of $\operatorname{Gal}\left(\widetilde{F}_{\infty} / F\right)$ of finite index. Moreover since the free part $\operatorname{Gal}(\widetilde{F} / F)$ of $\operatorname{Gal}\left(\widetilde{F}_{\infty} / F\right)$ is isomorphic to $\mathbb{Z}_{p}^{d+1+\delta_{F, p}}$, we may choose a basis $\left\{\gamma_{1}, \ldots, \gamma_{d+1+\delta_{F, p}}\right\}$ of $\operatorname{Gal}(\widetilde{F} / F)$ so that $\left\{\gamma_{1}^{p^{e_{1}}}, \ldots, \gamma_{d+1+\delta_{F, p}}^{e_{d+1+\delta_{F, p}}}\right\}$ forms a basis of the free part of $\mathfrak{G}$ for certain nonnegative integers $e_{1}, \ldots, e_{d+1+\delta_{F, p}}$ (due to elementary divisor theory). The Pontrjagin dual of $\left(\mathcal{A}_{\eta}^{\mathrm{CM}}\right)^{G_{K}}$ is then isomorphic to a certain quotient of $\Lambda_{\mathcal{O}}^{\mathrm{CM}} / J$, where $J$ is the ideal of $\Lambda_{\mathcal{O}}^{\mathrm{CM}}$ generated by $\left.\eta^{\text {gal }}\left(\gamma_{j}^{p^{e_{j}}}\right) \gamma_{j}^{p^{e_{j}}}\right|_{\widetilde{F}_{\infty}}-1$ for $1 \leq j \leq d+1+\delta_{F, p}$. Let $\varpi$ denote a uniformiser of $\mathcal{O}$. Then the $\Lambda_{\mathcal{O}}^{\mathrm{CM}}$-module $\Lambda_{\mathcal{O}}^{\mathrm{CM}} /\left(\varpi \Lambda_{\mathcal{O}}^{\mathrm{CM}}+J\right)$ is clearly finite, which implies that the height of $J$ is greater than or equal to $d+1+\delta_{F, p}$ (recall that the Krull dimension of $\Lambda_{\mathcal{O}}^{\mathrm{CM}}$ is $\left.d+2+\delta_{F, p}\right)$. The Pontrjagin dual of $\left(\mathcal{A}_{\eta}^{\mathrm{CM}}\right)^{G_{K}}$ is obviously annihilated by $J$, and it is thus pseudonull as a $\Lambda_{\mathcal{O}}^{\mathrm{CM}}$-module since $d$ is a positive integer.

Lemma 3.11. The local cohomology group $H^{1}\left(I_{K / F, w},\left(\mathcal{A}_{\eta}^{\mathrm{CM}}\right)^{I_{K, \tilde{w}_{0}}}\right)$ is copseudonull as a module over $\Lambda_{\mathcal{O}}^{\mathrm{CM}}$ for each place $w$ of $F$ in $S \backslash \Sigma_{p}$.

Proof. First we assume that $w$ is a place of $F$ contained in $\Sigma_{p}^{c}$. We shall prove that $\left(\mathcal{A}_{\eta}^{\mathrm{CM}}\right)^{I_{K, \tilde{w}_{0}}}$ is copseudonull as a $\Lambda_{\mathcal{O}}^{\mathrm{CM}}$-module, which immediately implies the desired conclusion on the local cohomology group at $w$. Since $K$ is a finite extension of $F$ and $w$ is totally ramified in the cyclotomic extension $F\left(\mu_{p^{\infty}}\right) / F\left(\mu_{p^{m_{w}}}\right)$ for a certain nonnegative integer $m_{w}$, the image of $I_{K, \tilde{w}_{0}}$ in $\operatorname{Gal}\left(F\left(\mu_{p^{\infty}}\right) / F\right)$ is infinite. We take an element $x$ of $I_{K, \tilde{w}_{0}}$ whose image in $\operatorname{Gal}\left(F\left(\mu_{p^{\infty}}\right) / F\right)$ is of infinite order. Let $F_{\infty}^{(w)} / F$ be the composite of 
all $\mathbb{Z}_{p}$-extensions over $F$ unramified outside $w$, and take an element $y$ of $I_{K, \tilde{w}_{0}}$ whose image in $\operatorname{Gal}\left(F_{\infty}^{(w)} / F\right)$ is of infinite order. Comparing the ramification at $w$, one readily sees that $F\left(\mu_{p \infty}\right)$ and $F_{\infty}^{(w)}$ are linearly disjoint over $F$. The Pontrjagin dual of $\left(\mathcal{A}_{\eta}^{\mathrm{CM}}\right)^{I_{K, \tilde{w}_{0}}}$ is then isomorphic to a certain quotient of $\Lambda_{\mathcal{O}}^{\mathrm{CM}} / J_{w}$, where $J_{w}$ is an ideal of $\Lambda_{\mathcal{O}}^{\mathrm{CM}}$ generated by $\left.\eta^{\text {gal }}(x) x\right|_{\widetilde{F}_{\infty}}-1$ and $\left.\eta^{\text {gal }}(y) y\right|_{\widetilde{F}_{\infty}}-1$. Furthermore the linear disjointness of $F\left(\mu_{p}\right)$ and $F_{\infty}^{(w)}$ over $F$ implies that $\left.\eta^{\text {gal }}(x) x\right|_{\widetilde{F}_{\infty}}-1$ and $\left.\eta^{\text {gal }}(y) y\right|_{\widetilde{F}_{\infty}}-1$ forms a regular sequence in $\Lambda_{\mathcal{O}}^{\mathrm{CM}}$. The height of $J_{w}$, which is contained in the annihilator ideal of $\left(\mathcal{A}_{\eta}^{\mathrm{CM}}\right)^{I_{K, \tilde{w}}}$, thus equals two, and hence $\left(\mathcal{A}_{\eta}^{\mathrm{CM}}\right)^{I_{K, w_{0}}}$ is copseudonull as a $\Lambda_{\mathcal{O}}^{\mathrm{CM}}$-module.

Next assume that $w$ is a place of $F$ contained in $S$ but not lying above $p$, and let $\ell$ denote the residue characteristic at $w$. The inertia subgroup $I_{K, \tilde{w}_{0}}$ acts on $\mathcal{A}_{\eta}^{\mathrm{CM}}$ trivially because $\widetilde{F}_{\infty} / F$ is unramified at $w$.

The definition of the branch character $\psi$ implies that $\eta^{\text {gal }} \psi^{-1}$ is ramified only at places lying above $p$. Hence $K$ is unramified outside $p$ over the field $K_{\psi}$ corresponding to the kernel of $\psi$, and the inertia subgroup $I_{K / F, w}$ of $\operatorname{Gal}(K / F)$ at $w$ is naturally regarded as that of $\operatorname{Gal}\left(K_{\psi} / F\right)$. Under this identification the inertia subgroup $I_{K / F, w}$ acts on $\mathcal{A}_{\eta}^{\mathrm{CM}}$ via the composition $I_{K / F, w} \hookrightarrow \operatorname{Gal}\left(K_{\psi} / F\right) \stackrel{\psi}{\longrightarrow} \mathcal{O}^{\times}$.

We shall prove that $H^{1}\left(I_{K / F, w}, \mathcal{A}_{\eta}^{\mathrm{CM}}\right)$ is trivial. When $w$ is unramified in the finite abelian extension $K / F$, the inertia subgroup $I_{K / F, w}$ is trivial and there is nothing to prove in this case. Hence we assume that $w$ is ramified in $K / F$. The restriction of $\psi$ to $I_{K / F, w}$ is then not trivial since $\psi: \operatorname{Gal}\left(K_{\psi} / F\right) \rightarrow \overline{\mathbb{Q}}^{\times}$is injective by definition. Let $I_{K / F, w}^{\mathrm{w}}$ be the $\ell$-Sylow subgroup (the wild part) of $I_{K / F, w}$ and $I_{K / F, w}^{\mathrm{t}}$ the tame quotient $I_{K / F, w} / I_{K / F, w}^{\mathrm{w}}$ of $I_{K / F, w}$. Note that the cohomology group $H^{1}\left(I_{K / F, w}^{\mathrm{w}}, \mathcal{A}_{\eta}^{\mathrm{CM}}\right)$ is trivial because it is annihilated by the cardinality of $I_{K / F, w}^{\mathrm{w}}$, which is relatively prime to $p$. By the inflation-restriction exact sequence

$$
0 \rightarrow H^{1}\left(I_{K / F, w}^{\mathrm{t}},\left(\mathcal{A}_{\eta}^{\mathrm{CM}}\right)^{I_{K / F, w}^{\mathrm{w}}}\right) \rightarrow H^{1}\left(I_{K / F, w}, \mathcal{A}_{\eta}^{\mathrm{CM}}\right) \rightarrow H^{1}\left(I_{K / F, w}^{\mathrm{w}}, \mathcal{A}_{\eta}^{\mathrm{CM}}\right)^{I_{K / F, w}^{\mathrm{t}}}
$$

combined with the triviality of $H^{1}\left(I_{K / F, w}^{\mathrm{w}}, \mathcal{A}_{\eta}^{\mathrm{CM}}\right)$, we can identify $H^{1}\left(I_{K / F, w}, \mathcal{A}_{\eta}^{\mathrm{CM}}\right)$ with $H^{1}\left(I_{K / F, w}^{\mathrm{t}},\left(\mathcal{A}_{\eta}^{\mathrm{CM}}\right)^{I_{K / F, w}^{\mathrm{w}}}\right)$. Therefore it suffices to verify that $H^{1}\left(I_{K / F, w}^{\mathrm{t}},\left(\mathcal{A}_{\eta}^{\mathrm{CM}}\right)^{I_{K / F}^{\mathrm{w}}, w}\right)$ is trivial in order to prove the vanishing of $H^{1}\left(I_{K / F}, \mathcal{A}_{\eta}^{\mathrm{CM}}\right)$.

If the action of $I_{K / F, w}^{\mathrm{w}}$ on $\mathcal{A}_{\eta}^{\mathrm{CM}}$ is not trivial, there exists an element $z$ of $I_{K / F, w}^{\mathrm{w}}$ such that $\psi^{\text {gal }}(z)-1$ does not equal zero. Since $\psi^{\text {gal }}(z)$ is a nontrivial $\ell$-power root of unity in $\mathcal{O}$, we easily see that $\psi^{\text {gal }}(z)-1$ is a nontrivial unit of $\mathcal{O}$.

By definition $\left(\mathcal{A}_{\eta}^{\mathrm{CM}}\right)^{I_{K / F, w}^{\mathrm{w}}}$ is annihilated by the unit $\psi^{\mathrm{gal}}(z)-1$ of $\mathcal{O}$, and hence it is trivial. This obviously implies the triviality of $H^{1}\left(I_{K / F, w}^{\mathrm{t}},\left(\mathcal{A}_{\eta}^{\mathrm{CM}}\right)^{I_{K / F, w}^{\mathrm{w}}}\right)$. If $I_{K / F, w}^{\mathrm{w}}$ acts trivially on $\mathcal{A}_{\eta}^{\mathrm{CM}}$, the nontriviality of the action of $I_{K / F, w}$ on $\mathcal{A}_{\eta}^{\mathrm{CM}}$ implies that the the tame quotient $I_{K / F, w}^{\mathrm{t}}$ acts nontrivially on $\mathcal{A}_{\eta}^{\mathrm{CM}}$. In other words, if we denote a generator of the cyclic group $I_{K / F, w}^{\mathrm{t}}$ by $z^{\prime}, \psi^{\text {gal }}\left(z^{\prime}\right)-1$ is a nonzero element of $\Lambda_{\mathcal{O}}^{\mathrm{CM}}$. The first cohomology group $H^{1}\left(I_{K / F, w}^{\mathrm{t}}, \mathcal{A}_{\eta}^{\mathrm{CM}}\right)$ of the finite cyclic group $I_{K / F, w}^{\mathrm{t}}$ is described as $\mathcal{A}_{\eta}^{\mathrm{CM}} /\left(\psi^{\mathrm{gal}}\left(z^{\prime}\right)-\right.$ 1) $\mathcal{A}_{\eta}^{\mathrm{CM}}$, and we thus deduce its triviality from the divisibility of $\mathcal{A}_{\eta}^{\mathrm{CM}}$ as a $\Lambda_{\mathcal{O}}^{\mathrm{CM}}$-module.

The diagram (3.3) combined with Lemmata 3.10 and 3.11 implies that both the kernel and the cokernel of the natural map $\operatorname{Sel}_{\mathcal{A}_{\eta}^{\mathrm{CM}}}^{\Sigma} \rightarrow \operatorname{Ker}\left(\phi_{F_{S} / K}\right)$ are copseudonull $\Lambda_{\mathcal{O}}^{\mathrm{CM}}$-modules. In particular, the characteristic ideals of Pontrjagin duals of these $\Lambda_{\mathcal{O}}^{\mathrm{CM}}$-modules coincide 
with each other. Now we investigate the kernel of $\phi_{F_{S} / K}$ more precisely, by generalising Greenberg's arguments made around the equation (7) in Gr94 to multi-variable cases. For this purpose we prepare the following technical lemma.

Lemma 3.12. Let $\mathcal{G}$ be a profinite group and $\rho: \mathcal{G} \rightarrow \mathcal{O}^{\times}$a continuous character, where $\mathcal{O}$ denotes the ring of integers of a finite extension of $\mathbb{Q}_{p}$. We denote by $\mathcal{T}_{\rho}$ a continuous $\mathcal{G}$-representation $\mathcal{O}(\rho) \otimes_{\mathcal{O}} \mathcal{O}[[\Gamma]]^{\sharp}$ equipped with the diagonal action of $\mathcal{G}$, and by $\mathcal{A}_{\rho}$ its associated discrete $\mathcal{G}$-representation defined by $\mathcal{T}_{\rho} \otimes_{\mathcal{O}[[\Gamma]]} \mathcal{O}[[\Gamma]]^{\vee}$.

Suppose that $\mathcal{G}$ admits a free abelian pro-p quotient $\Gamma$ of finite $\mathbb{Z}_{p}$-rank and that the kernel of the natural surjection $\mathcal{G} \rightarrow \Gamma$ is contained in the kernel of $\rho$.

Then for an arbitrary free pro-p subgroup $\Gamma^{\prime}$ of $\Gamma$, the first cohomology group $H^{1}\left(\Gamma^{\prime}, \mathcal{A}_{\rho}\right)$ is trivial.

Note that, in the statement of Lemma 3.12, the free abelian quotient $\Gamma$ naturally acts on $\mathcal{A}_{\rho}$ thanks to the assumption on the kernel of $\rho$.

Proof. We verify the claim by induction on the $\mathbb{Z}_{p}$-rank $n$ of $\Gamma^{\prime}$. First consider the case where $n$ equals 1 . Then if we choose an element $\tilde{\gamma}$ of $\mathcal{G}$ so that its image $\gamma$ in $\Gamma$ topologically generates $\Gamma^{\prime}$, the first cohomology group $H^{1}\left(\Gamma^{\prime}, \mathcal{A}_{\rho}\right)$ is described as the quotient $\mathcal{A}_{\rho} /(\rho(\tilde{\gamma}) \gamma-1) \mathcal{A}_{\rho}$. Therefore the claim holds since $\mathcal{A}_{\rho}$ is a divisible $\mathcal{O}[[\Gamma]]$-module and $\rho(\tilde{\gamma}) \gamma-1$ is a nonzero element of $\mathcal{O}[[\Gamma]]$.

For general $n$, let us choose an arbitrary free pro- $p$ subgroup $\Gamma^{\prime \prime}$ of $\Gamma^{\prime}$ of $\mathbb{Z}_{p}$-rank $n-1$, and consider the inflation-restriction exact sequence

$$
0 \longrightarrow H^{1}\left(\Gamma^{\prime} / \Gamma^{\prime \prime}, \mathcal{A}_{\rho}^{\Gamma^{\prime \prime}}\right) \longrightarrow H^{1}\left(\Gamma^{\prime}, \mathcal{A}_{\rho}\right) \longrightarrow H^{1}\left(\Gamma^{\prime \prime}, \mathcal{A}_{\rho}\right)^{\Gamma^{\prime} / \Gamma^{\prime \prime}}
$$

The induction hypothesis implies the triviality of $H^{1}\left(\Gamma^{\prime \prime}, \mathcal{A}_{\rho}\right)$. We readily see that the cohomology group $H^{1}\left(\Gamma^{\prime} / \Gamma^{\prime \prime}, \mathcal{A}_{\rho}^{\Gamma^{\prime \prime}}\right)$ is also trivial; indeed, since the $\Gamma^{\prime \prime}$-coinvariant $\left(\mathcal{T}_{\rho}\right)_{\Gamma^{\prime \prime}}$ of $\mathcal{T}_{\rho}$ is isomorphic to $\mathcal{O}\left[\left[\Gamma / \Gamma^{\prime \prime}\right]\right]$ as an $\mathcal{O}\left[\left[\Gamma / \Gamma^{\prime \prime}\right]\right]$-module, its Pontrjagin dual $\mathcal{A}_{\rho}^{\Gamma^{\prime \prime}}$ is a divisible $\mathcal{O}\left[\left[\Gamma / \Gamma^{\prime \prime}\right]\right]$-module. Therefore $H^{1}\left(\Gamma^{\prime} / \Gamma^{\prime \prime}, \mathcal{A}_{\rho}^{\Gamma^{\prime \prime}}\right)$ vanishes by the same reason as the case where $n$ equals 1 . Consequently the exact sequence (3.4) implies that the cohomology group $H^{1}\left(\Gamma^{\prime}, \mathcal{A}_{\rho}\right)$ is also trivial .

Applying Lemma 3.12 to both the source and the target of the local-to-global morphism $\phi_{F_{S} / K}$, we obtain the following corollaries.

Corollary 3.13. The restriction map induces an isomorphism between $H^{1}\left(F_{S} / K, \mathcal{A}_{\eta}^{\mathrm{CM}}\right)$ and $H^{1}\left(F_{S} / \widetilde{K}_{\infty}^{\mathrm{CM}}, \mathcal{A}_{\eta}^{\mathrm{CM}}\right)^{\widetilde{\Gamma}}$.

Here we identify $\widetilde{\Gamma}=\operatorname{Gal}(\widetilde{F} / F)$ with the Galois group $\operatorname{Gal}\left(\widetilde{K}_{\infty}^{\mathrm{CM}} / K\right)$ in the canonical manner.

Proof. Due to the inflation-restriction exact sequence

$$
0 \longrightarrow H^{1}\left(\widetilde{\Gamma}, \mathcal{A}_{\eta}^{\mathrm{CM}}\right) \longrightarrow H^{1}\left(F_{S} / K, \mathcal{A}_{\eta}^{\mathrm{CM}}\right) \longrightarrow H^{1}\left(F_{S} / \widetilde{K}_{\infty}^{\mathrm{CM}}, \mathcal{A}_{\eta}^{\mathrm{CM}}\right)^{\widetilde{\Gamma}} \longrightarrow H^{2}\left(\widetilde{\Gamma}, \mathcal{A}_{\eta}^{\mathrm{CM}}\right)
$$

it suffices to show that $H^{i}\left(\widetilde{\Gamma}, \mathcal{A}_{\eta}^{\mathrm{CM}}\right)$ is trivial for $i=1,2$ (note that $\operatorname{Gal}\left(F_{S} / \widetilde{K}_{\infty}^{\mathrm{CM}}\right)$ acts trivially on $\mathcal{A}_{\eta}^{\mathrm{CM}}$ ). The first cohomology vanishes by a direct consequence of Lemma 3.12, The second cohomology vanishes since $\widetilde{\Gamma}$ is a free abelian pro- $p$ group and thus its cohomological dimension is equal to or less than 1 . This completes the proof. 
Corollary 3.14. Let $w$ be a place of $F$ contained in $S \backslash \Sigma_{p}$. Then for each place $\tilde{w}$ of $K$ lying above $w$, the restriction morphism

$$
H^{1}\left(I_{K, \tilde{w}}, \mathcal{A}_{\eta}^{\mathrm{CM}}\right) \rightarrow \prod_{\hat{w} \mid \tilde{w}} H^{1}\left(I_{\widetilde{K}_{\infty}^{\mathrm{CM}}, \hat{w}}, \mathcal{A}_{\eta}^{\mathrm{CM}}\right)
$$

is injective (here $I_{\widetilde{K}_{\infty}^{\mathrm{CM}}, \hat{w}}$ denotes the inertia subgroup of $\operatorname{Gal}\left(F_{S} / \widetilde{K}_{\infty}^{\mathrm{CM}}\right)$ at $\left.\hat{w}\right)$.

Proof. By the inflation-restriction exact sequence, we see that the kernel of the restriction map (3.5) is isomorphic to $H^{1}\left(I\left(\widetilde{K}_{\infty}^{\mathrm{CM}} / K\right)_{w}, \mathcal{A}_{\eta}^{\mathrm{CM}}\right)$, where we denotes by $I\left(\widetilde{K}_{\infty}^{\mathrm{CM}} / K\right)_{w}$ the inertia subgroup of $\operatorname{Gal}\left(\widetilde{K}_{\infty}^{\mathrm{CM}} / K\right)$ at $w$. Since $I\left(\widetilde{K}_{\infty}^{\mathrm{CM}} / K\right)_{w}$ is regarded as a $\mathbb{Z}_{p}$-submodule of the free $\mathbb{Z}_{p}$-module $\widetilde{\Gamma} \cong \operatorname{Gal}\left(\widetilde{K}_{\infty}^{\mathrm{CM}} / K\right)$ of finite rank, it is also free as a $\mathbb{Z}_{p}$-module. Applying Lemma 3.12 to $H^{1}\left(I\left(\widetilde{K}_{\infty}^{\mathrm{CM}} / K\right)_{\tilde{w}}, \mathcal{A}_{\eta}^{\mathrm{CM}}\right)$, we conclude that it is trivial.

Corollaries 3.13 and 3.14 imply that the kernel of $\phi_{F_{S} / K}$ is isomorphic to that of

$$
H^{1}\left(F_{S} / \widetilde{K}_{\infty}^{\mathrm{CM}}, \mathcal{A}_{\eta}^{\mathrm{CM}}\right)^{\Delta \times \widetilde{\Gamma}} \rightarrow \prod_{w \in S \backslash \Sigma_{p}} \prod_{\hat{w} \mid w} H^{1}\left(I_{\widetilde{K}_{\infty}^{\mathrm{CM}}, \hat{w}}, \mathcal{A}_{\eta}^{\mathrm{CM}}\right)
$$

or in other words, the kernel of the restriction map

$$
\phi_{F_{S} / \widetilde{K}_{\infty}^{\mathrm{CM}}}: \operatorname{Hom}_{\Delta \times \widetilde{\Gamma}}\left(\operatorname{Gal}\left(F_{S} / \widetilde{K}_{\infty}^{\mathrm{CM}}\right)^{\mathrm{ab}}, \mathcal{A}_{\eta}^{\mathrm{CM}}\right) \rightarrow \prod_{w \in S \backslash \Sigma_{p}} \prod_{\hat{w} \mid w} \operatorname{Hom}\left(I_{\widetilde{K}_{\infty}^{\mathrm{ab}}, \hat{w}}, \mathcal{A}_{\eta}^{\mathrm{CM}}\right) .
$$

Let $M_{\Sigma_{p}}$ denote the maximal abelian pro- $p$ extension of $\widetilde{K}_{\infty}^{\mathrm{CM}}$ unramified outside places above $\Sigma_{p}$, and let $X_{\Sigma_{p}}$ denote the Galois group of $M_{\Sigma_{p}} / \widetilde{K}_{\infty}^{\mathrm{CM}}$. Then as in classical Iwasawa theory, an element $g$ of $\operatorname{Gal}\left(\widetilde{K}_{\infty}^{\mathrm{CM}} / F\right)$ acts on $X_{\Sigma_{p}}$ by $x \mapsto{ }^{g} x:=\tilde{g} x \tilde{g}^{-1}$, where $\tilde{g}$ denotes an arbitrary lift of $g$ to $\operatorname{Gal}\left(M_{\Sigma_{p}} / F\right)$. Furthermore we define the maximal $\psi$-isotypic quotient $X_{\Sigma_{p},(\psi)}$ of $X_{\Sigma_{p}}$ as

$$
X_{\Sigma_{p},(\psi)}=\left(X_{\Sigma_{p}} \otimes_{\mathbb{Z}_{p}} \mathcal{O}\right) \otimes_{\mathcal{O}[\Delta]} \mathcal{O}\left[\Delta^{\mathrm{cyc}}\right] .
$$

Here the second tensor product is defined with respect to the map

$$
\mathcal{O}[\Delta] \rightarrow \mathcal{O}\left[\Delta^{\text {cyc }}\right] ;\left.\delta \mapsto \psi(\delta) \delta\right|_{F\left(\mu_{p}\right)} \quad \text { for } \delta \text { in } \Delta=\operatorname{Gal}(K / F)
$$

(recall that $\Delta^{\text {cyc }}$ is defined as $\operatorname{Gal}\left(F\left(\mu_{p}\right) / F\right)$ ). Since an element $\delta$ of $\Delta$ acts on $\mathcal{A}_{\eta}^{\mathrm{CM}}$ via the multiplication by $\left.\psi(\delta) \delta\right|_{F\left(\mu_{p}\right)}$, the kernel of $\phi_{F_{S} / \widetilde{K}_{\infty}^{\mathrm{CM}}}$ is calculated as

$$
\begin{aligned}
\operatorname{Ker}\left(\phi_{F_{S} / \widetilde{K}_{\infty}^{\mathrm{CM}}}\right) & =\operatorname{Hom}_{\Delta \times \Gamma}\left(X_{\Sigma_{p}}, \mathcal{A}_{\eta}^{\mathrm{CM}}\right)=\operatorname{Hom}_{\mathcal{O}[[\Delta \times \Gamma]]}\left(X_{\Sigma_{p}} \otimes_{\mathbb{Z}_{p}} \mathcal{O}, \mathcal{A}_{\eta}^{\mathrm{CM}}\right) \\
& \cong \operatorname{Hom}_{\Lambda_{\mathcal{O}}^{\mathrm{CM}}}\left(X_{\Sigma_{p},(\psi)}, \mathcal{A}_{\eta}^{\mathrm{CM}}\right) .
\end{aligned}
$$

In order to investigate the structure of $\operatorname{Hom}_{\Lambda_{\mathcal{O}}^{\mathrm{CM}}}\left(X_{\Sigma_{p},(\psi)}, \mathcal{A}_{\eta}^{\mathrm{CM}}\right)$, we introduce the notion of twisting of finitely generated $\Lambda_{\mathcal{O}}^{\mathrm{CM}}$-module. Recall that we have defined the $\rho$-twisting map

$$
\operatorname{Tw}_{\rho}: \Lambda_{\mathcal{O}}^{\mathrm{CM}} \stackrel{\sim}{\rightarrow} \Lambda_{\mathcal{O}}^{\mathrm{CM}} ; g \mapsto \rho(g) g \quad \text { for } g \text { in } \operatorname{Gal}\left(\widetilde{F}_{\infty} / F\right)
$$

for an arbitrary continuous character $\rho: \operatorname{Gal}\left(\widetilde{F}_{\infty} / F\right) \rightarrow \mathcal{O}^{\times}$in Section 1, For an arbitrary $\Lambda_{\mathcal{O}}^{\mathrm{CM}}$-module $M$, we define the $\rho$-twist $\operatorname{Tw}_{\rho}(M)$ of $M$ as the $\mathcal{O}$-module $M$ on which an element $r$ of $\Lambda_{\mathcal{O}}^{\mathrm{CM}}$ acts as the scalar multiplication by $\mathrm{Tw}_{\rho}(r)$. The following lemma describes basic properties of twisting of $\Lambda_{\mathcal{O}}^{\mathrm{CM}}$-modules.

Lemma 3.15. Let $\rho: \operatorname{Gal}\left(\widetilde{F}_{\infty} / F\right) \rightarrow \mathcal{O}^{\times}$be a continuous character and $M$ a $\Lambda_{\mathcal{O}}^{\mathrm{CM}}-$ module. 
(1) The Pontrjagin dual $\left(\mathrm{Tw}_{\rho}(M)\right)^{\vee}$ of $\operatorname{Tw}_{\rho}(M)$ is isomorphic to $\mathrm{Tw}_{\rho}\left(M^{\vee}\right)$, the $\rho$-twist of the Pontrjagin dual $M^{\vee}$ of $M$, as a $\Lambda_{\mathcal{O}}^{\mathrm{CM}}$-module.

(2) For an arbitrary $\Lambda_{\mathcal{O}}^{\mathrm{CM}}$-module $N$, we obtain an equality of $\Lambda_{\mathcal{O}}^{\mathrm{CM}}$-modules

$$
\operatorname{Hom}_{\Lambda_{\mathcal{O}}^{\mathrm{CM}}}\left(\operatorname{Tw}_{\rho}(M), N\right)=\operatorname{Hom}_{\Lambda_{\mathcal{O}}^{\mathrm{CM}}}\left(M, \mathrm{Tw}_{\rho^{-1}}(N)\right) .
$$

(3) Assume that $M$ is finitely generated and torsion as a $\Lambda_{\mathcal{O}}^{\mathrm{CM}}-$ module. Then $\mathrm{Tw}_{\rho}(M)$ is pseudonull if and only if $M$ itself is pseudonull. Furthermore we obtain the following equality of ideals of $\Lambda_{\mathcal{O}}^{\mathrm{CM}}$ :

$$
\operatorname{Char}_{\Lambda_{\mathcal{O}}^{\mathrm{CM}}}\left(\operatorname{Tw}_{\rho}(M)\right)=\operatorname{Tw}_{\rho^{-1}}\left(\operatorname{Char}_{\Lambda_{\mathcal{O}}^{\mathrm{CM}}}(M)\right)
$$

In the statement of Lemma 3.15(3), the characteristic ideals of finitely generated torsion modules over the semilocal Iwasawa algebra $\Lambda_{\mathcal{O}}^{\mathrm{CM}}$ are defined componentwisely. We shall recall the precise definition of them in Definition 3.22. Note that the Pontrjagin dual $M^{\vee}=\operatorname{Hom}_{\mathrm{cts}}\left(M, \mathbb{Q}_{p} / \mathbb{Z}_{p}\right)$ of a $\Lambda_{\mathcal{O}}^{\mathrm{CM}}$-module $M$ is equipped with the $\Lambda_{\mathcal{O}}^{\mathrm{CM}}$-module structure defined by $r \phi(x):=\phi(r x)$, which is incompatible with the usual action of $\operatorname{Gal}\left(\widetilde{F}_{\infty} / F\right)$ on $\operatorname{Hom}_{\text {cts }}\left(M, \mathbb{Q}_{p} / \mathbb{Z}_{p}\right)$ defined by $g \phi(x):=\phi\left(g^{-1} x\right)$.

Proof. The assertions (1) and (2) directly follow from the definition of $p$-twisting. For the assertion (3), assume that $M$ is a pseudonull module. By definition there exist distinct nonzero elements $r_{1}$ and $r_{2}$ in the annihilator ideal of $M$ which are relatively prime to each other. One then readily observes that both $\mathrm{Tw}_{\rho^{-1}}\left(r_{1}\right)$ and $\mathrm{Tw}_{\rho^{-1}}\left(r_{2}\right)$ annihilate $\mathrm{Tw}_{\rho}(M)$ and are relatively prime to each other, which implies that $\operatorname{Tw}_{\rho}(M)$ is also pseudonull. Similarly one readily verifies the converse implication.

Now let us consider the statement on characteristic ideals in the assertion (3). We first reduce the claim to the case where $M$ is an elementary $\Lambda_{\mathcal{O}}^{\mathrm{CM}}$-module of the form $\Lambda_{\mathcal{O}}^{\mathrm{CM}} /(a)$, by considering componentwisely and using the structure theorem of finitely generated torsion $\mathcal{O}[[\operatorname{Gal}(\widetilde{F} / F)]]-$ modules. Then the reduced claim obviously holds since there exists an isomorphism

$$
\Lambda_{\mathcal{O}}^{\mathrm{CM}} /\left(\mathrm{Tw}_{\rho^{-1}}(a)\right) \stackrel{\sim}{\rightarrow} \mathrm{Tw}_{\rho}\left(\Lambda_{\mathcal{O}}^{\mathrm{CM}} /(a)\right) ; r \quad \bmod \left(\operatorname{Tw}_{\rho^{-1}}(a)\right) \mapsto \mathrm{Tw}_{\rho}(r) \quad \bmod (a)
$$

of $\Lambda_{\mathcal{O}}^{\mathrm{CM}}$-modules.

Since $\mathcal{A}_{\eta}^{\mathrm{CM}}$ is isomorphic to $\mathrm{Tw}_{\eta^{\text {gal }} \psi^{-1}}\left(\Lambda_{\mathcal{O}}^{\mathrm{CM}, \vee}\right)$ as a $\Lambda_{\mathcal{O}}^{\mathrm{CM}}$-module, we may calculate by using Lemma 3.15 the kernel of $\phi_{F_{S} / \widetilde{K}_{\infty}^{\mathrm{CM}}}$ as

$$
\begin{aligned}
\operatorname{Ker}\left(\phi_{F_{S} / \widetilde{K}_{\infty}^{\mathrm{CM}}}\right) & =\operatorname{Hom}_{\Lambda_{\mathcal{O}}^{\mathrm{CM}}}\left(X_{\Sigma_{p},(\psi)}, \operatorname{Tw}_{\eta^{\mathrm{gal}} \psi^{-1}}\left(\Lambda_{\mathcal{O}}^{\mathrm{CM}}\right)^{\vee}\right) \\
& \cong \operatorname{Hom}_{\Lambda_{\mathcal{O}}^{\mathrm{CM}}}\left(\operatorname{Tw}_{\eta^{\mathrm{gal}} \psi^{-1}}\left(\Lambda_{\mathcal{O}}^{\mathrm{CM}}\right), X_{\Sigma_{p},(\psi)}^{\vee}\right) \\
& =\operatorname{Hom}_{\Lambda_{\mathcal{O}}^{\mathrm{CM}}}\left(\Lambda_{\mathcal{O}}^{\mathrm{CM}}, \operatorname{Tw}_{\eta^{\mathrm{gal},-1} \psi}\left(X_{\Sigma_{p},(\psi)}\right)^{\vee}\right) \cong \operatorname{Tw}_{\eta^{\mathrm{gal},-1} \psi}\left(X_{\Sigma_{p},(\psi)}\right)^{\vee} .
\end{aligned}
$$

As a consequence we obtain the following proposition.

Proposition 3.16. The Pontrjagin dual of the Selmer group $\operatorname{Sel}_{\mathcal{A}_{\eta}^{C M}}^{\Sigma}$ is pseudoisomorphic to $\mathrm{Tw}_{\eta^{\text {gal },-1} \psi}\left(X_{\Sigma_{p},(\psi)}\right)$ as a $\Lambda_{\mathcal{O}}^{\mathrm{CM}}-$ module. In particular, we obtain the following equality among characteristic ideals:

$$
\operatorname{Char}_{\Lambda_{\mathcal{O}}^{\mathrm{CM}}} \operatorname{Sel}_{\mathcal{A}_{\eta}^{\mathrm{CM}}}^{\mathrm{LV}}=\operatorname{Char}_{\Lambda_{\mathcal{O}}^{\mathrm{CM}}}\left(\operatorname{Tw}_{\eta^{\mathrm{gal},-1} \psi}\left(X_{\Sigma_{p},(\psi)}\right)\right)=\operatorname{Tw}_{\eta^{\mathrm{gal}} \psi^{-1}}\left(\operatorname{Char}_{\Lambda_{\mathcal{O}}^{\mathrm{CM}}} X_{\Sigma_{p},(\psi)}\right) .
$$

Remark 3.17. We can define $\Lambda_{\mathcal{O}}^{\mathrm{CM}}$-modules $\mathcal{T}_{\psi}^{\mathrm{CM}}$ and $\mathcal{A}_{\psi}^{\mathrm{CM}}$ similarly to $\mathcal{T}_{\eta}^{\mathrm{CM}}$ and $\mathcal{A}_{\eta}^{\mathrm{CM}}$ : namely, we set $\mathcal{T}_{\psi}^{\mathrm{CM}}=\mathcal{O}(\psi) \otimes_{\mathcal{O}} \Lambda_{\mathcal{O}}^{\mathrm{CM}, \sharp}$ and $\mathcal{A}_{\psi}^{\mathrm{CM}}=\mathcal{T}_{\psi}^{\mathrm{CM}} \otimes_{\Lambda_{\mathcal{O}}^{\mathrm{CM}}} \Lambda_{\mathcal{O}}^{\mathrm{CM}, \vee}$. Then we readily 
verify that the Pontrjagin dual of the Selmer group $\operatorname{Sel}_{\mathcal{A}_{\psi}^{\mathrm{CM}}}^{\Sigma}$ associated to $\mathcal{A}_{\psi}^{\mathrm{CM}}$ and the maximal $\psi$-isotypic quotient $X_{\Sigma_{p},(\psi)}$ of $X_{\Sigma_{p}} \otimes_{\mathbb{Z}_{p}} \mathcal{O}$ are pseudoisomorphic as $\Lambda_{\mathcal{O}}^{\mathrm{CM}}$-modules by the same argument as above (and they are isomorphic when the cardinality of $\Delta$ is relatively prime to $p$ ). In other words, $\operatorname{Sel}_{\mathcal{A}_{\eta}^{\mathrm{CM}}}^{\Sigma}$ is copseudoisomorphic to $\operatorname{Tw}_{\eta^{\text {gal },-1} \psi}\left(\operatorname{Sel}_{\mathcal{A}_{\psi}^{\mathrm{CM}}}^{\Sigma}\right)$ as a $\Lambda_{\mathcal{O}}^{\mathrm{CM}}$-module.

We have defined the finitely generated $\Lambda_{\mathcal{O}}^{\text {CM }}$-module $X_{\Sigma_{p}} \otimes_{\mathbb{Z}_{p}} \mathcal{O}$ in a similar way to the manner where one defines the (classical) Iwasawa module $X_{\{p\}}$ : the Galois group $\operatorname{Gal}\left(M_{\{p\}} / \mathrm{K}\left(\mu_{p \infty}\right)\right)$ of the maximal abelian pro- $p$ extension $M_{\{p\}}$ of $\mathrm{K}\left(\mu_{p} \infty\right)$ which is unramified outside places lying above $p$ (here $\mathrm{K}$ denotes an algebraic number field). Proposition 3.16 describes the relation between Selmer groups (of Greenberg type) and Iwasawa modules constructed in a classical way. The algebraic structure of the Iwasawa module $X_{\Sigma_{p},(\psi)}$ has been thoroughly studied by Perrin-Riou PR81] (for imaginary quadratic fields) and Hida and Tilouine [HT94] (for general CM fields).

3.2. Exact control theorem. We shall prove in this subsection the exact control theorem (Theorem $(\mathrm{B})$ for Selmer groups $\operatorname{Sel}_{\mathcal{A}_{\eta}^{\mathrm{CM}}}^{\Sigma}$ associated to a größencharacter $\eta$ of type $\left(A_{0}\right)$ defined on a CM number field $F$ (see Definition [3.6). Recall that the Iwasawa algebra $\Lambda_{\mathcal{O}}^{\mathrm{CM}}=\mathcal{O}\left[\left[\operatorname{Gal}\left(\widetilde{F}_{\infty} / F\right)\right]\right]$ is a semilocal ring each of whose components is isomorphic to the ring of formal power series over $\mathcal{O}$ in $d+1+\delta_{F, p}$ variables. Therefore there exists a regular sequence $\left\{x_{1}, \ldots, x_{d+\delta_{F, p}}\right\}$ in $\Lambda_{\mathcal{O}}^{\mathrm{CM}}$ such that each $x_{j}$ belongs to the kernel $\mathfrak{A}^{\text {cyc }}$ of the augmentation map $\Lambda_{\mathcal{O}}^{\mathrm{CM}} \rightarrow \Lambda_{\mathcal{O}}^{\text {cyc }}=\mathcal{O}\left[\left[\operatorname{Gal}\left(F\left(\mu_{p^{\infty}}\right) / F\right)\right]\right]$. Such a regular sequence $\left\{x_{1}, \ldots, x_{d+\delta_{F, p}}\right\}$ is contained in the Jacobson radical of $\Lambda_{\mathcal{O}}^{\mathrm{CM}}$, and thus its arbitrary permutation is also regular. For each $j$ with $0 \leq j \leq d+\delta_{F, p}$, let $\mathfrak{A}_{j}$ denote the ideal of $\Lambda_{\mathcal{O}}^{\mathrm{CM}}$ generated by $x_{1}, \ldots, x_{j}$ :

$$
\mathfrak{A}_{j}=\left(x_{1}, \ldots, x_{j}\right) .
$$

As the convention we use the symbol $\mathfrak{A}_{0}$ for the zero ideal of $\Lambda_{\mathcal{O}}^{\mathrm{CM}}$. Let $\mathcal{A}_{\eta}^{\mathrm{CM}}\left[\mathfrak{A}_{j}\right]$ denote the $\Lambda_{\mathcal{O}}^{\mathrm{CM}}$-submodule of $\mathcal{A}_{\eta}^{\mathrm{CM}}$ consisting of all the elements annihilated by $\mathfrak{A}_{j}$.

We here introduce the following nontriviality condition $(\mathrm{ntr})_{\mathfrak{P}}$ on $\mathcal{A}_{\eta}^{\mathrm{CM}}$ for each place $\mathfrak{P}$ of $F$ lying above $p$ :

$(\mathbf{n t r})_{\mathfrak{P}}$ : for each maximal ideal $\mathfrak{M}$ of the semilocal Iwasawa algebra $\Lambda_{\mathcal{O}}^{\mathrm{CM}}$, the maximal $\mathfrak{M}$-torsion submodule $\mathcal{A}_{\eta}^{\mathrm{CM}}[\mathfrak{M}]$ of $\mathcal{A}_{\eta}^{\mathrm{CM}}$ is not trivial as a $D_{\mathfrak{P}}$-module.

Theorem 3.18 (Exact control theorem, Theorem B). Assume that the nontriviality condition (ntr) $)_{\mathfrak{P}}$ is fulfilled for every place $\mathfrak{P}$ contained in $\Sigma_{p}^{c}$. Let $\left\{x_{1}, \ldots, x_{d+\delta_{F, p}}\right\}$ be an arbitrary regular sequence in $\Lambda_{\mathcal{O}}^{\mathrm{CM}}$ all of whose elements belong to $\mathfrak{A}^{\mathrm{cyc}}$, and let us define $\mathfrak{A}_{j}$ as the ideal of $\Lambda_{\mathcal{O}}^{\mathrm{CM}}$ generated by $x_{1}, x_{2}, \ldots, x_{j}$. Then the natural map

$$
\mathrm{Sel}_{\mathcal{A}_{\eta}^{\mathrm{CM}}\left[\mathfrak{A}_{j}\right]}^{\Sigma} \longrightarrow \mathrm{Sel}_{\mathcal{A}_{\eta}^{\mathrm{CM}}}^{\Sigma}\left[\mathfrak{A}_{j}\right]
$$

induced by the natural inclusion $\mathcal{A}_{\eta}^{\mathrm{CM}}\left[\mathfrak{A}_{j}\right] \hookrightarrow \mathcal{A}_{\eta}^{\mathrm{CM}}$ is an isomorphism for each $j$ with $0 \leq j \leq d+\delta_{F, p}$

For the proof of Theorem 3.18, we first replace our Selmer group $\operatorname{Sel}_{\mathcal{A}_{\eta}^{\mathrm{CM}}}^{\Sigma}$ by the strict Selmer group $\mathrm{Sel}_{\mathcal{A}_{\eta}^{\mathrm{CM}}}^{\mathrm{s} \text {,str }}$ defined below. We then control the strict Selmer group under specialisation with respect to the regular sequence $\left\{x_{1}, \ldots, x_{j}\right\}$. 
Definition 3.19. For each $j$ with $0 \leq j \leq d+\delta_{F, p}$, we define the strict Selmer group $\mathrm{Sel}_{\mathcal{A}_{\eta}^{\mathrm{CM}}\left[\mathfrak{A}_{j}\right]}^{\Sigma, \mathrm{str}}$ as the kernel of the global-to-local map

$$
H^{1}\left(F_{S} / F, \mathcal{A}_{\eta}^{\mathrm{CM}}\left[\mathfrak{A}_{j}\right]\right) \longrightarrow \prod_{\substack{\lambda \in S \\ \lambda \nmid p \infty}} H^{1}\left(I_{\lambda}, \mathcal{A}_{\eta}^{\mathrm{CM}}\left[\mathfrak{A}_{j}\right]\right) \times \prod_{\mathfrak{P} \in \Sigma_{p}^{c}} H^{1}\left(F_{\mathfrak{P}}, \mathcal{A}_{\eta}^{\mathrm{CM}}\left[\mathfrak{A}_{j}\right]\right)
$$

Since, for the strict Selmer group $\operatorname{Sel}_{\mathcal{A}_{\eta}^{\mathrm{CM}}\left[\mathfrak{H}_{j}\right]}^{\Sigma, \mathrm{str}}$, only the local conditions at places contained in $\Sigma_{p}^{c}$ are modified compared to the definition of the usual Selmer group $\operatorname{Sel}_{\mathcal{A}_{\eta}^{\mathrm{CM}\left[\mathfrak{A}_{j}\right]}}^{\Sigma}$ in Definition 3.6, we have the following comparison result:

Lemma 3.20. Let the notation be as in Theorem 3.18 and assume that the condition $(\mathrm{ntr})_{\mathfrak{P}}$ is fulfilled for each place $\mathfrak{P}$ contained in $\Sigma_{p}^{c}$. Then for every $0 \leq j \leq d+\delta_{F, p}$ the natural injection $\mathrm{Sel}_{\mathcal{A}_{\eta}^{\mathrm{CM}}\left[\mathfrak{A}_{j}\right]}^{\Sigma, \mathrm{str}} \hookrightarrow \operatorname{Sel}_{\mathcal{A}_{\eta}^{\mathrm{CM}}\left[\mathfrak{A}_{j}\right]}^{\Sigma}$ induces an isomorphism.

Proof of Lemma 3.20. We consider the following commutative diagram:

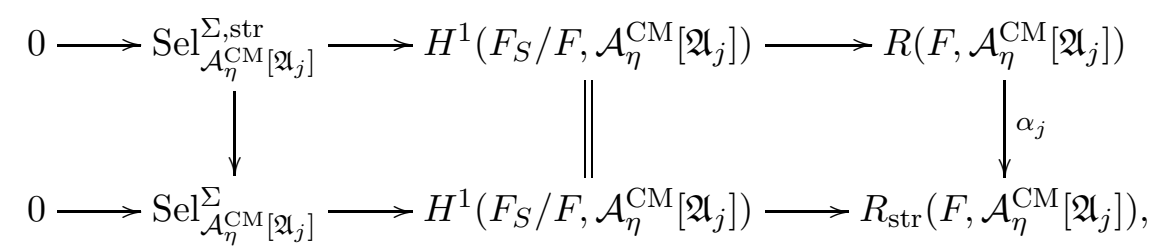

where we use the following abbreviation on the direct products of local cohomology groups:

$$
\begin{aligned}
R\left(F, \mathcal{A}_{\eta}^{\mathrm{CM}}\left[\mathfrak{A}_{j}\right]\right)= & \prod_{\substack{\lambda \in S \\
\lambda \nmid p \infty}} H^{1}\left(I_{\lambda}, \mathcal{A}_{\eta}^{\mathrm{CM}}\left[\mathfrak{A}_{j}\right]\right) \times \prod_{\mathfrak{P} \in \Sigma_{p}^{c}} H^{1}\left(I_{\mathfrak{P}}, \mathcal{A}_{\eta}^{\mathrm{CM}}\left[\mathfrak{A}_{j}\right]\right), \\
R_{\mathrm{str}}\left(F, \mathcal{A}_{\eta}^{\mathrm{CM}}\left[\mathfrak{A}_{j}\right]\right)= & \prod_{\substack{\lambda \in S \\
\lambda \in p \infty}} H^{1}\left(I_{\lambda}, \mathcal{A}_{\eta}^{\mathrm{CM}}\left[\mathfrak{A}_{j}\right]\right) \times \prod_{\mathfrak{P} \in \Sigma_{p}^{c}} H^{1}\left(F_{\mathfrak{P}}, \mathcal{A}_{\eta}^{\mathrm{CM}}\left[\mathfrak{A}_{j}\right]\right) .
\end{aligned}
$$

The right vertical map $\alpha_{j}$ of the diagram (3.7) is defined as the usual restriction maps on the $\Sigma_{p}^{c}$-components and the identity maps on the other components. The snake lemma implies that the natural map $\operatorname{Sel}_{\mathcal{A}_{\eta}^{\mathrm{CM}}\left[\mathfrak{A}_{j}\right]}^{\Sigma, \mathrm{sr}} \rightarrow \operatorname{Sel}_{\mathcal{A}_{\eta}^{\mathrm{CM}\left[\mathfrak{A}_{j}\right]}}^{\Sigma}$ is an injection whose cokernel is isomorphic to a certain submodule of $\operatorname{Ker}\left(\alpha_{j}\right)$. On the other hand, $\operatorname{Ker}\left(\alpha_{j}\right)$ is isomorphic to $\prod_{\mathfrak{P} \in \Sigma_{p}^{c}} H^{1}\left(D_{\mathfrak{P}} / I_{\mathfrak{P}}, \mathcal{A}_{\eta}^{\mathrm{CM}}\left[\mathfrak{A}_{j}\right]^{I_{\mathfrak{P}}}\right)$ due to the inflation-restriction sequence.

We now verify that each component $H^{1}\left(D_{\mathfrak{P}} / I_{\mathfrak{P}}, \mathcal{A}_{\eta}^{\mathrm{CM}}\left[\mathfrak{A}_{j}\right]^{I_{\mathfrak{P}}}\right)$ of the direct product above is trivial. Since the the Pontrjagin dual $\left(\mathcal{A}_{\eta}^{\mathrm{CM}}\right)^{\vee}$ of $\mathcal{A}_{\eta}^{\mathrm{CM}}$ is isomorphic to $\Lambda_{\mathcal{O}}^{\mathrm{CM}}$ as a $\Lambda_{\mathcal{O}}^{\mathrm{CM}}$ module, we have $\left(\mathcal{A}_{\eta}^{\mathrm{CM}}\left[\mathfrak{A}_{j}\right]^{I_{\mathfrak{P}}}\right)^{\vee} \cong \Lambda_{\mathcal{O}} / J_{\mathfrak{P}}$ with the annihilator ideal $J_{\mathfrak{P}}$ of $\left(\mathcal{A}_{\eta}^{\mathrm{CM}}\left[\mathfrak{A}_{j}\right]^{I_{\mathfrak{P}}}\right)^{\vee}$. We denote by $f_{\mathfrak{P}}$ the value of the Frobenius element at $\mathfrak{P}$ acting on $\mathcal{A}_{\eta}^{\mathrm{CM}}\left[\mathfrak{A}_{j}\right]^{I_{\mathfrak{P}}}$. Then the unramified cohomology group $H^{1}\left(D_{\mathfrak{P}} / I_{\mathfrak{P}}, \mathcal{A}_{\eta}^{\mathrm{CM}}\left[\mathfrak{A}_{j}\right]^{I_{\mathfrak{P}}}\right)$ is described as the quotient $\mathcal{A}_{\eta}^{\mathrm{CM}}\left[\mathfrak{A}_{j}\right]^{I_{\mathfrak{P}}} /\left(f_{\mathfrak{P}}-1\right) \mathcal{A}_{\eta}^{\mathrm{CM}}\left[\mathfrak{A}_{j}\right]^{I_{\mathfrak{P}}}$, and hence it is trivial if and only if the multiplication by $f_{\mathfrak{P}}-1$ on $\mathcal{A}_{\eta}^{\mathrm{CM}}\left[\mathfrak{A}_{j}\right]^{I_{\mathfrak{P}}}$ is surjective; in other words, it is trivial if and only if the multiplication by $f_{\mathfrak{P}}-1$ induces an injective endomorphism on $\Lambda_{\mathcal{O}}^{\mathrm{CM}} / J_{\mathfrak{P}}$. The latter condition is obviously fulfilled when $f_{\mathfrak{P}}-1$ is invertible in $\Lambda_{\mathcal{O}}^{\mathrm{CM}} / J_{\mathfrak{P}}$, or equivalently, when it is invertible in $\Lambda_{\mathcal{O}}^{\mathrm{CM}} / \mathfrak{M}$ for each maximal ideal of $\Lambda_{\mathcal{O}}^{\mathrm{CM}}$ containing $J_{\mathfrak{P}}$. The condition (ntr) Th $_{\mathfrak{P}}$ thus implies the triviality of each cohomology group $H^{1}\left(D_{\mathfrak{P}} / I_{\mathfrak{P}}, \mathcal{A}_{\eta}^{\mathrm{CM}}\left[\mathfrak{A}_{j}\right]^{I_{\mathfrak{P}}}\right)$ since the condition $(\mathrm{ntr})_{\mathfrak{P}}$ asserts that the value of $f_{\mathfrak{P}}$ does not equal 1. Consequently, the strict Selmer group 
$\mathrm{Sel}_{\mathcal{A}_{\eta}^{\mathrm{CM}\left[\mathfrak{A}_{j}\right]}}^{\Sigma, \mathrm{str}}$ is isomorphic to $\operatorname{Sel}_{\mathcal{A}_{\eta}^{\mathrm{CM}}\left[\mathfrak{A}_{j}\right]}^{\Sigma}$ for every $j$ with $0 \leq j \leq d+\delta_{F, p}$ when we assume the condition $(\mathrm{ntr})_{\mathfrak{P}}$ for each place $\mathfrak{P}$ contained in $\Sigma_{p}^{c}$.

Let us return to the proof of Theorem 3.18.

Proof of Theorem 3.18 . In order to control the strict Selmer group $\operatorname{Sel}_{\mathcal{A}_{\eta}^{\mathrm{CM}}\left[\mathfrak{A}_{j}\right]}^{\Sigma, \mathrm{str}}$, we consider the following diagram for each $j$ :

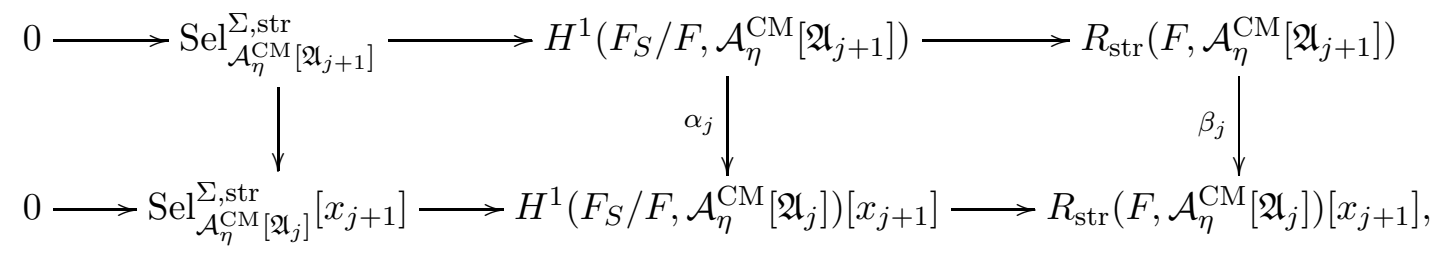

where the symbol $R_{\text {str }}\left(F, \mathcal{A}_{\eta}^{\mathrm{CM}}\left[\mathfrak{A}_{j}\right]\right)$ denotes the groups introduced in the diagram (3.7). The middle and right vertical maps $\alpha_{j}, \beta_{j}$ are induced from the long exact sequence in Galois cohomology associated to the following short exact sequence of $\operatorname{Gal}\left(F_{S} / F\right)$-modules:

$$
0 \rightarrow \mathcal{A}_{\eta}^{\mathrm{CM}}\left[\mathfrak{A}_{j+1}\right] \rightarrow \mathcal{A}_{\eta}^{\mathrm{CM}}\left[\mathfrak{A}_{j}\right] \stackrel{\times x_{j+1}}{\longrightarrow} \mathcal{A}_{\eta}^{\mathrm{CM}}\left[\mathfrak{A}_{j}\right] \rightarrow 0 .
$$

In particular $\alpha_{j}$ is surjective by construction and it suffices to verify that the map $\alpha_{j}$ (resp. $\beta_{j}$ ) is injective in order to prove that the map $\operatorname{Sel}_{\mathcal{A}_{\eta}^{\mathrm{CM}\left[\mathfrak{A}_{j+1}\right]}}^{\Sigma} \rightarrow \operatorname{Sel}_{\mathcal{A}_{\eta}^{\mathrm{CM}\left[\mathfrak{A}_{j}\right]}}^{\Sigma}\left[x_{j+1}\right]$ in consideration is injective (resp. surjective) by the snake lemma applied on (3.8).

As for the kernel of $\alpha_{j}$, we first observe that it is isomorphic to the quotient module $H^{0}\left(F_{S} / F, \mathcal{A}_{\eta}^{\mathrm{CM}}\left[\mathfrak{A}_{j}\right]\right) / x_{j+1} H^{0}\left(F_{S} / F, \mathcal{A}_{\eta}^{\mathrm{CM}}\left[\mathfrak{A}_{j}\right]\right)$ by the long exact sequence of cohomology of $\operatorname{Gal}\left(F_{S} / F\right)$ obtained from (3.9). Obviously the global zeroth cohomology group $H^{0}\left(F_{S} / F, \mathcal{A}_{\eta}^{\mathrm{CM}}\left[\mathfrak{A}_{j}\right]\right)$ is a submodule of the local zeroth cohomology group $H^{0}\left(F_{\mathfrak{P}}, \mathcal{A}_{\eta}^{\mathrm{CM}}\left[\mathfrak{A}_{j}\right]\right)$ for an arbitrary place $\mathfrak{P}$ in $\Sigma_{p}^{c}$. As we shall see in the next paragraph, the latter cohomology group $H^{0}\left(F_{\mathfrak{P}}, \mathcal{A}_{\eta}^{\mathrm{CM}}\left[\mathfrak{A}_{j}\right]\right)$ is trivial under the condition $(\mathrm{ntr})_{\mathfrak{P}}$. This implies that $\operatorname{Ker}\left(\alpha_{j}\right)$ is trivial.

Next we verify the triviality of the kernel of $\beta_{j}$. For each place $w$ in $S$, let $\beta_{j, w}$ denote the map induced by $\beta_{j}$ on the $w$-component of $R_{\mathrm{str}}\left(F, \mathcal{A}_{\eta}^{\mathrm{CM}}\left[\mathfrak{A}_{j+1}\right]\right)$. Then by the long exact sequence of group cohomology for $D_{w^{-}}$or $I_{w^{-}}$-modules associated to the short exact sequence (3.9), we have

$$
\operatorname{Ker}\left(\beta_{j, w}\right) \cong \begin{cases}H^{0}\left(F_{w}, \mathcal{A}_{\eta}^{\mathrm{CM}}\left[\mathfrak{A}_{j}\right]\right) / x_{j+1} H^{0}\left(F_{w}, \mathcal{A}_{\eta}^{\mathrm{CM}}\left[\mathfrak{A}_{j}\right]\right) & \text { for } w \in \Sigma_{p}^{c}, \\ H^{0}\left(I_{w}, \mathcal{A}_{\eta}^{\mathrm{CM}}\left[\mathfrak{A}_{j}\right]\right) / x_{j+1} H^{0}\left(I_{w}, \mathcal{A}_{\eta}^{\mathrm{CM}}\left[\mathfrak{A}_{j}\right]\right) & \text { for } w \in S \backslash\left(\Sigma_{p} \cup \Sigma_{p}^{c} \cup \Sigma_{\infty}\right) .\end{cases}
$$

For a place $w$ in $\Sigma_{p}^{c}$, the cohomology group $H^{0}\left(F_{w}, \mathcal{A}_{\eta}^{\mathrm{CM}}\left[\mathfrak{A}_{j}\right]\right)$ itself is trivial under the condition $(\mathrm{ntr})_{w}$. In fact, it is easy to redescribe $H^{0}\left(F_{w}, \mathcal{A}_{\eta}^{\mathrm{CM}}\left[\mathfrak{A}_{j}\right]\right)$ as $H^{0}\left(D_{w} / I_{w}, \mathcal{A}_{\eta}^{\mathrm{CM}}\left[\mathfrak{A}_{j}\right]^{I_{w}}\right)$ by definition. We denote by $f_{w}$ the value of the Frobenius element at $w$ acting on $\mathcal{A}_{\eta}^{\mathrm{CM}}\left[\mathfrak{A}_{j}\right]^{I_{w}}$. Then, as in the proof of Lemma 3.20, we readily see that the Pontrjagin dual of $H^{0}\left(D_{w} / I_{w}, \mathcal{A}_{\eta}^{\mathrm{CM}}\left[\mathfrak{A}_{j}\right]^{I_{w}}\right)$ is isomorphic to the cokernel of the multiplication of $f_{w}-1$ on $\Lambda_{\mathcal{O}}^{\mathrm{CM}} / J_{w}$, where $J_{w}$ is the annihilator ideal of $\left(\mathcal{A}_{\eta}^{\mathrm{CM}}\left[\mathfrak{A}_{j}\right]^{I_{w}}\right)^{\vee}$. The element $f_{w}-1$ is, however, a unit of $\Lambda_{\mathcal{O}}^{\mathrm{CM}} / J_{w}$ thanks to the condition (ntr) $)_{w}$ as discussed in the proof of Lemma 3.20, and in particular the cokernel of the multiplication of $f_{w}-1$ is trivial. This completes the proof of the triviality of the kernel of $\beta_{j, w}$.

For a place $w$ in $S \backslash\left(\Sigma_{p} \cup \Sigma_{p}^{c} \cup \Sigma_{\infty}\right)$, the inertia subgroup $I_{w}$ acts on $\mathcal{O}(\eta)$ through a finite quotient and acts on $\Lambda_{\mathcal{O}}^{\mathrm{CM}, \sharp}$ trivially. Let $E_{w}$ denote the (finite) image of $I_{w}$ under the Galois 
character $\eta^{\text {gal }}$ and let $\varpi^{n_{w}} \mathcal{O}$ denote the ideal of $\mathcal{O}$ generated by every element of the form $\zeta-1$ with $\zeta$ belonging to $E_{w}$ (here $\varpi$ denotes a uniformiser of $\mathcal{O}$ ). Then one readily sees that the cohomology group $H^{0}\left(I_{w}, \mathcal{A}_{\eta}^{\mathrm{CM}}\left[\mathfrak{A}_{j}\right]\right)$ is none other than the maximal $\varpi^{n_{w}}$-torsion submodule of $\mathcal{A}_{\eta}^{\mathrm{CM}}\left[\mathfrak{A}_{j}\right]$. The Pontrjagin dual of $\operatorname{Ker}\left(\beta_{j, w}\right)$ is thus isomorphic to the kernel of the multiplication of $x_{j+1}$ on $\Lambda_{\mathcal{O}}^{\mathrm{CM}} /\left(\varpi^{n_{w}}, x_{1}, \ldots, x_{j}\right)$. The sequence $x_{1}, \ldots, x_{j+1}, \varpi^{n_{w}}$ is, however, a regular sequence and contained in the Jacobson radical of $\Lambda_{\mathcal{O}}^{\mathrm{CM}}$, and thus its permutation $\varpi^{n_{w}}, x_{1}, \ldots, x_{j+1}$ is also a regular sequence in $\Lambda_{\mathcal{O}}^{\mathrm{CM}}$. We therefore see that the kernel of $\beta_{j, w}$ is trivial.

We finally remark that, by applying the same arguments as the proof of Lemma 3.20 to the discrete $\Lambda_{\mathcal{O}}^{\mathrm{CM}}$-module $\mathcal{A}_{\psi}^{\mathrm{CM}}$, we obtain the following result.

Lemma 3.21. The natural injection $\mathrm{Sel}_{\mathcal{A}_{\psi}^{\mathrm{CM}}}^{\Sigma \text {,str }} \hookrightarrow \mathrm{Sel}_{\mathcal{A}_{\psi}^{\mathrm{CM}}}^{\Sigma}$ is a copseudoisomorphism of discrete $\Lambda_{\mathcal{O}}^{\mathrm{CM}}$-modules. Furthermore, if the following condition $(\mathrm{ntr})_{\psi, \mathfrak{P}}$ on $\mathcal{A}_{\psi}^{\mathrm{CM}}$ is fulfilled for each place $\mathfrak{P} \in \Sigma_{p}^{c}$, the injection above is an isomorphism.

(ntr) $)_{\psi \mathfrak{P}}$ : For each maximal ideal $\mathfrak{M}$ of $\Lambda_{\mathcal{O}}^{\mathrm{CM}}$, the maximal $\mathfrak{M}$-torsion submodule $\mathcal{A}_{\psi}^{\mathrm{CM}}[\mathfrak{M}]$ of $\mathcal{A}_{\psi}^{\mathrm{CM}}$ is not trivial as a $D_{\mathfrak{P}}$-module.

Proof. We may verify that $\left(\mathcal{A}_{\psi}^{\mathrm{CM}}\right)^{I_{\mathfrak{P}}}$ is a copseudonull $\Lambda_{\mathcal{O}}^{\mathrm{CM}}$-module for each place $\mathfrak{P}$ of $F$ in $\Sigma_{p}^{c}$ by arguments similar to the proof of the $\Lambda_{\mathcal{O}}^{\mathrm{CM}}$-copseudonullity of $\left(\mathcal{A}_{\eta}^{\mathrm{CM}}\right)^{I_{\mathfrak{P}}}$; see the proof of Lemma 3.11. Therefore the first half of the claim is true because the cokernel of the natural injection $\mathrm{Sel}_{\mathcal{A}_{\psi}^{\mathrm{CM}}}^{\Sigma \text {,str }} \hookrightarrow \mathrm{Sel}_{\mathcal{A}_{\psi}^{\mathrm{CM}}}^{\Sigma}$ is a submodule of the direct product of unramified cohomology groups $H^{1}\left(D_{\mathfrak{P}} / I_{\mathfrak{P}},\left(\mathcal{A}_{\psi}^{\mathrm{CM}}\right)^{I_{\mathfrak{P}}}\right)$ for places $\mathfrak{P}$ in $\Sigma_{p}^{c}$.

The second half of the claim is verified by the same argument as the proof of Lemma 3.20 ,

3.3. Greenberg's criterion for almost divisibility. As is well known, the characteristic ideal of a finitely generated torsion module over a complete noetherian regular local domain is not necessarily preserved under basechange (or specialisation) procedures. Indeed the existence of a nontrivial pseudonull submodule causes peculiar behaviour of the characteristic ideal under specialisation. Therefore, when we discuss specialisation of the multi-variable Iwasawa main conjecture (or, in particular, specialisation of multi-variable Selmer groups), it is crucial to check whether the Pontrjagin dual of the Selmer group contains nontrivial pseudonull submodules or not. Greenberg has recently presented in Gr] certain sufficient conditions for the pseudonull submodule of the Pontrjagin dual of the Selmer group to be trivial, which is applicable to quite general situations. In this subsection we introduce various hypotheses which are necessary to state Greenberg's criterion, and then we briefly review the main results of $\mathrm{Gr}$.

3.3.1. Algebraic settings. Let $\Lambda_{0}$ be the ring $\mathcal{O}\left[\left[T_{1}, \ldots, T_{m}\right]\right]$ of formal power series over the ring of integers $\mathcal{O}$ of a finite extension of $\mathbb{Q}_{p}$, and let $\mathcal{R}$ be a $\Lambda_{0}$-algebra which is isomorphic to the direct product of a finite number of copies of $\Lambda_{0}$. For each cofinitely generated discrete $R$-module $\mathcal{A}$, we define the $\mathcal{R}$-corank $\operatorname{corank}_{\mathcal{R}}(\mathcal{A})$ of $\mathcal{A}$ as a finite set $\left(\operatorname{corank}_{\mathcal{R}_{i}}\left(e_{i} \mathcal{A}\right)\right)_{i \in I}$ of nonnegative integers, where each $\mathcal{R}_{i}$ denotes a local component of the semilocal ring $\mathcal{R}$ cut out by an idempotent $e_{i}$. We endow the set of the coranks of cofinitely generated discrete $\mathcal{R}$-modules with the componentwise partial order; namely, the notation $\operatorname{corank}_{\mathcal{R}}\left(\mathcal{A}_{1}\right) \leq \operatorname{corank}_{\mathcal{R}}\left(\mathcal{A}_{2}\right)$ means that $\operatorname{corank}_{\mathcal{R}_{i}}\left(e_{i} \mathcal{A}_{1}\right) \leq \operatorname{corank}_{\mathcal{R}_{i}}\left(e_{i} \mathcal{A}_{2}\right)$ holds for every $i$ in $I$. The characteristic ideal of a finitely generated torsion $\mathcal{R}$-module for the semilocal ring $\mathcal{R}$ is also defined componentwisely as follows. 
Definition 3.22 (Characteristic ideal of $\mathcal{R}$-modules). Let $M$ be a finitely generated torsion $\mathcal{R}$-module. Then we define the $\left(\mathcal{R}\right.$-) characteristic ideal $\operatorname{Char}_{\mathcal{R}}(M)$ of $M$ as the ideal of $\mathcal{R}$ corresponding to $\prod_{i \in I} \operatorname{Char}_{\mathcal{R}_{i}}\left(e_{i} M\right)$ under the indecomposable decomposition $\mathcal{R}=$ $\prod_{i \in I} \mathcal{R}_{i}$ of $\mathcal{R}$. Here $\operatorname{Char}_{\mathcal{R}_{i}}\left(e_{i} M\right)$ denotes the characteristic ideal of the finitely generated torsion module $e_{i} M$ over the regular local ring $\mathcal{R}_{i}$, which is defined in the usual manner.

By definition the characteristic ideal $\operatorname{Char}_{\mathcal{R}}(M)$ is a principal ideal of $\mathcal{R}$.

Remark 3.23. In Gr10] and $\mathrm{Gr}$, Greenberg assumes that the coefficient ring $\mathcal{R}$ is a local ring equipped with several good properties (which Greenberg calls a reflexive ring). In our setting the coefficient ring $\mathcal{R}$ is a semilocal ring and is no longer local. However each local component of $\mathcal{R}$ is a regular local ring isomorphic to $\Lambda_{0}$, which is compatible with Greenberg's setting. Indeed it is not difficult at all to extend Greenberg's results of [Gr10] and $[\mathrm{Gr}$ to our semilocal coefficient case by using the componentwise decomposition of the coefficient ring $\mathcal{R}$ and $\mathcal{R}$-modules. In Section 3.3 .3 we shall introduce statements of Greenberg's results of [Gr10] and [Gr] extended to the semilocal coefficient case.

Now let K, $S$ and $\mathcal{T}$ be as in Section 3.1.1. Since the semilocal ring $\mathcal{R}=\prod_{i \in I} \mathcal{R}_{i}$ satisfies all the conditions introduced in Section 3.1.1, we can consider the Selmer group of $\mathcal{R}$-linear $G_{\mathrm{K}}$-representations. For the discrete $\mathcal{R}$-module $\mathcal{A}=\mathcal{T} \otimes_{\mathcal{R}} \mathcal{R}^{\vee}$ associated to $\mathcal{T}$, we specify an $\mathcal{R}$-submodule $L\left(\mathrm{~K}_{v}, \mathcal{A}\right)$ of the local Galois cohomology group $H^{1}\left(\mathrm{~K}_{v}, \mathcal{A}\right)$ for each $v$ in $S$, which we call a local condition at $v$. We denote such a specification of local conditions by $\mathcal{L}$ for brevity. Set $Q_{\mathcal{L}}\left(\mathrm{K}_{v}, \mathcal{A}\right)$ as the quotient $H^{1}\left(\mathrm{~K}_{v}, \mathcal{A}\right) / L\left(\mathrm{~K}_{v}, \mathcal{A}\right)$ for each $v$ in $S$. We define $L(\mathrm{~K}, \mathcal{A})$ as the product of the $\mathcal{R}$-submodules $L\left(\mathrm{~K}_{v}, \mathcal{A}\right)$ for all places $v$ in $S$, and similarly we introduce notation on products of local cohomology groups as follows:

$$
P(\mathrm{~K}, \mathcal{A})=\prod_{v \in S} H^{1}\left(\mathrm{~K}_{v}, \mathcal{A}\right), \quad Q_{\mathcal{L}}(\mathrm{K}, \mathcal{A})=\prod_{v \in S} Q_{\mathcal{L}}\left(\mathrm{K}_{v}, \mathcal{A}\right) .
$$

The $\mathcal{L}$-Selmer group $\operatorname{Sel}_{\mathcal{L}}(\mathrm{K}, \mathcal{A})$ associated to $\mathcal{A}$ is defined to be the kernel of the natural global-to-local homomorphism

$$
\phi_{\mathcal{L}}: H^{1}\left(\mathrm{~K}_{S} / \mathrm{K}, \mathcal{A}\right) \rightarrow Q_{\mathcal{L}}(\mathrm{K}, \mathcal{A})
$$

induced by restriction morphisms of Galois cohomologies. By definition, $\operatorname{Sel}_{\mathcal{L}}(\mathrm{K}, \mathcal{A})$ is an $\mathcal{R}$-submodule of the first cohomology group $H^{1}\left(\mathrm{~K}_{S} / \mathrm{K}, \mathcal{A}\right)$ of the Galois group $\operatorname{Gal}\left(\mathrm{K}_{S} / \mathrm{K}\right)$ with coefficients in $\mathcal{A}$. When we take the trivial specification $\mathcal{L}_{\text {triv }}$, or in other words, when we impose the minimal local condition $L\left(\mathrm{~K}_{v}, \mathcal{A}\right)=0$ on each place $v$ in $S$, the $\mathcal{L}_{\text {triv }}$-Selmer group is denoted by $\amalg^{1}(\mathrm{~K}, S, \mathcal{A})$ in $[\mathrm{Gr} 06, \mathrm{Gr}$ ] and referred as the fine $S$-Selmer group associated to $\mathcal{A}$ after Coates and Sujatha CS05].

Concerning the algebraic structure of the $\mathcal{L}$-Selmer groups, the following two statements are known to be equivalent (see [Gr06, Proposition 2.4] for the proof):

- the Pontrjagin dual $\operatorname{Sel}_{\mathcal{L}}^{\vee}(\mathrm{K}, \mathcal{A})$ of the $\mathcal{L}$-Selmer group $\operatorname{Sel}_{\mathcal{L}}(\mathrm{K}, \mathcal{A})$ does not contain nontrivial $\Lambda_{0}$-pseudonull submodules;

- the $\mathcal{L}$-Selmer group $\operatorname{Sel}_{\mathcal{L}}(\mathrm{K}, \mathcal{A})$ is almost divisible as a discrete $\Lambda_{0}$-module; that is, the equality $\mathfrak{P S e l}_{\mathcal{L}}(\mathrm{K}, \mathcal{A})=\operatorname{Sel}_{\mathcal{L}}(\mathrm{K}, \mathcal{A})$ holds for all but finitely many prime ideals $\mathfrak{P}$ of height one of $\Lambda_{0}$.

3.3.2. Various hypotheses. Greenberg has thoroughly studied almost $\Lambda_{0}$-divisibility of the $\mathcal{L}$-Selmer group and established certain useful criteria for almost $\Lambda_{0}$-divisibility in Gr06, Gr10, Gr. Now let us introduce various hypotheses which are necessary to state Greenberg's criteria (see also [Gr, Section 2.1]). 
The first two hypotheses concern the Kummer (or Cartier) dual $\mathcal{T}^{*}=\operatorname{Hom}_{\mathrm{cts}}\left(\mathcal{A}, \mu_{p^{\infty}}\right)$ of $\mathcal{A}$. For a place $v$ contained in $S$, we consider the following two statements:

$\left(\mathbf{L O C}{ }_{\mathcal{A}, v}^{(1)}\right)$ : the local Galois invariant submodule $H_{\mathrm{cts}}^{0}\left(\mathrm{~K}_{v}, \mathcal{T}^{*}\right)$ of $\mathcal{T}^{*}$ is trivial; $\left(\mathbf{L O C}_{\mathcal{A}, v}^{(2)}\right)$ : the quotient module $\mathcal{T}^{*} / H_{\text {cts }}^{0}\left(\mathrm{~K}_{v}, \mathcal{T}^{*}\right)$ is reflexive as an $\mathcal{R}$-module.

The next hypothesis concerns the generalised second Tate- ̌́afarevič group $\amalg^{2}(\mathrm{~K}, S, \mathcal{A})$ defined as the kernel of the global-to-local morphism

$$
H^{2}\left(\mathrm{~K}_{S} / \mathrm{K}, \mathcal{A}\right) \rightarrow \prod_{v \in S} H^{2}\left(\mathrm{~K}_{v}, \mathcal{A}\right)
$$

induced by usual restriction morphisms. Then we consider:

$\left(\mathbf{L E O} \mathbf{A}_{\mathcal{A}}\right)$ : the generalised second Tate-ŠSafarevič group $\amalg^{2}(\mathrm{~K}, S, \mathcal{A})$ is cotorsion as an $\mathcal{R}$-module.

One of the significant features concerning the hypothesis $\left(\mathrm{LEO}_{\mathcal{A}}\right)$ is that it behaves well under specialisation procedures with respect to height-one prime ideals; namely, the condition $\left(\operatorname{LEO}_{\mathcal{A}}\right)$ holds if and only if the condition $\left(\operatorname{LEO}_{\mathcal{A}[\Pi]}\right)$ holds (as a condition on the $\Lambda_{0} /(\Pi)$-module $\left.\mathcal{A}[\Pi]\right)$ for all but finitely many prime ideals $(\Pi)$ of height one of $\Lambda_{0}$ (generated by a prime element $\Pi$ ). Here we denote by $\mathcal{A}[\Pi]$ the maximal $\Pi$-torsion submodule of $\mathcal{A}$. We refer to [Gr06, Lemma 4.4.1 and Remark 2.1.3] for the proof of this property.

Finally we introduce the hypothesis on the global-to-local morphism $\phi_{\mathcal{L}}$ :

$\left(\mathbf{S U R}_{\mathcal{A}, \mathcal{L}}\right)$ : the global-to-local morphism $\phi_{\mathcal{L}}$ is surjective.

3.3.3. Greenberg's criterion. The following criterion for the almost divisibility of $\operatorname{Sel}_{\mathcal{L}}(\mathrm{K}, \mathcal{A})$ is due to Greenberg $\left[\mathrm{Gr}\right.$. We state it for modules over the semilocal ring $\mathcal{R}=\prod_{i \in I} \mathcal{R}_{i}$, contrary to the settings in $\mathrm{Gr}$.

Theorem 3.24 ([Gr, Proposition 4.1.1]). Let $\mathcal{R}$ be a finite $\Lambda_{0}$-algebra which is isomorphic to the direct product of finitely many copies of $\Lambda_{0}$, and let $\mathrm{K}, S, \mathcal{A}$ be as above. Assume that the local condition $L(\mathrm{~K}, \mathcal{A})(\subset P(\mathrm{~K}, \mathcal{A}))$ is almost $\Lambda_{0}$-divisible. Suppose also that all the conditions $\left(\mathrm{LOC}_{\mathcal{A}, v_{0}}^{(1)}\right)$ (for a certain nonarchimedean place $v_{0}$ in $\left.S\right),\left(\mathrm{LOC}_{\mathcal{A}, v}^{(2)}\right)$ (for every place $v$ in $S),\left(\operatorname{LEO}_{\mathcal{A}}\right)$ and $\left(\mathrm{SUR}_{\mathcal{A}, \mathcal{L}}\right)$ are fulfilled.

Then the $\mathcal{L}$-Selmer group $\operatorname{Sel}_{\mathcal{L}}(\mathrm{K}, \mathcal{A})$ is almost divisible as an $\Lambda_{0}$-module.

Remark 3.25. Among various assumptions of Theorem 3.24, the almost $\Lambda_{0}$-divisibility of $L(\mathrm{~K}, \mathcal{A})$ and the latter two hypotheses $\left(\mathrm{LEO}_{\mathcal{A}}\right),\left(\mathrm{SUR}_{\mathcal{A}, \mathcal{L}}\right)$ are rather nontrivial and not so easy to verify. In fact, the hypothesis $\left(\operatorname{LEO}_{\mathcal{A}}\right)$ is closely related to the weak Leopoldt conjecture in classical settings and is quite nontrivial (see [Gr06, Introduction and Section 6.D] for further discussion and for several examples where the hypothesis $\left(\operatorname{LEO}_{\mathcal{A}}\right)$ is not valid). The surjectivity condition $\left(\mathrm{SUR}_{\mathcal{A}, \mathcal{L}}\right)$ of the global-to-local morphism $\phi_{\mathcal{L}}$ is closely related to the triviality of the dual Selmer group, as discussed later in Section 3.3.4. Finally, the local condition $L(\mathrm{~K}, \mathcal{A})$ often tends to be not almost divisible; even in our CM setting, the unramified cohomology group $H_{\mathrm{ur}}^{1}\left(F_{\mathfrak{P}^{c}}, \mathcal{A}_{\eta}^{\mathrm{CM}}\right)$ at a place $\mathfrak{P}^{c}$ in $\Sigma_{p}^{c}$ might not be almost divisible in general, and we cannot directly apply Greenberg's criterion to $\operatorname{Sel}_{\mathcal{A}_{\eta}^{\mathrm{CM}}}^{\Sigma}$. This is one of the reasons why we replace our Selmer group $\operatorname{Sel}_{\mathcal{A}_{\eta}^{\mathrm{CM}}}^{\Sigma}$ with the strict Selmer group $\mathrm{Sel}_{\mathcal{A}_{\eta}^{\mathrm{CM}}}^{\Sigma \text {,str }}$ in Section 3.4. 
3.3.4. Dual Selmer groups and the surjectivity hypothesis. By virtue of Poitou and Tate's long exact sequence on Galois cohomology groups [NSW00, (8.6.10)], the cokernel of the global-to-local homomorphism $\phi_{\mathcal{L}}$ is represented in terms of the dual Selmer group $\operatorname{Sel}_{\mathcal{L}^{*}}\left(\mathrm{~K}, \mathcal{T}^{*}\right)$ of $\operatorname{Sel}_{\mathcal{L}}(\mathrm{K}, \mathcal{A})$, which enables us to check the hypothesis $\left(\mathrm{SUR}_{\mathcal{A}, \mathcal{L}}\right)$ by investigating the triviality of $\operatorname{Sel}_{\mathcal{L}^{*}}\left(\mathrm{~K}, \mathcal{T}^{*}\right)$. We here define the dual Selmer group $\operatorname{Sel}_{\mathcal{L}^{*}}\left(\mathrm{~K}, \mathcal{T}^{*}\right)$ and introduce a criterion for its triviality, which is also due to Greenberg [Gr10]. In the following paragraphs the subscript "cts" denotes the Galois cohomology groups of continuous cocycles.

Let $\mathcal{T}^{*}=\operatorname{Hom}_{\text {cts }}\left(\mathcal{A}, \mu_{p^{\infty}}\right)$ denote the Kummer dual of $\mathcal{A}$. Then the natural pairing $\mathcal{A} \times \mathcal{T}^{*} \rightarrow \mu_{p^{\infty}}$ combined with the cup product of the Galois cohomology induces the local Tate pairing

$$
H^{1}\left(\mathrm{~K}_{v}, \mathcal{A}\right) \times H_{\text {cts }}^{1}\left(\mathrm{~K}_{v}, \mathcal{T}^{*}\right) \rightarrow \mathbb{Q}_{p} / \mathbb{Z}_{p}
$$

for each $v$ in $S$, which is a perfect pairing as is well known. We specify a subgroup $L^{*}\left(\mathrm{~K}_{v}, \mathcal{T}^{*}\right)$ of $H_{\text {cts }}^{1}\left(\mathrm{~K}_{v}, \mathcal{T}^{*}\right)$ as the orthogonal complement of $L\left(\mathrm{~K}_{v}, \mathcal{A}\right)$ under the local Tate pairing (3.11). We denote such specifications of submodules of the local Galois cohomology groups $H_{\text {cts }}^{1}\left(\mathrm{~K}_{v}, \mathcal{T}^{*}\right)$ by $\mathcal{L}^{*}$. The dual Selmer group $\operatorname{Sel}_{\mathcal{L}^{*}}\left(\mathrm{~K}, \mathcal{T}^{*}\right)$ for $\mathcal{T}^{*}$ is then defined as the kernel of the global-to-local homomorphism

$$
\phi_{\mathcal{L}^{*}}: H_{\mathrm{cts}}^{1}\left(\mathrm{~K}_{S} / \mathrm{K}, \mathcal{T}^{*}\right) \rightarrow Q_{\mathcal{L}^{*}}\left(\mathrm{~K}, \mathcal{T}^{*}\right),
$$

where $Q_{\mathcal{L}^{*}}\left(\mathrm{~K}, \mathcal{T}^{*}\right)$ is defined as the direct product $\prod_{v \in S} H_{\text {cts }}^{1}\left(\mathrm{~K}_{v}, \mathcal{T}^{*}\right) / L^{*}\left(\mathrm{~K}_{v}, \mathcal{T}^{*}\right)$. Meanwhile the fine $S$-Selmer group $\amalg^{1}\left(\mathrm{~K}, S, \mathcal{T}^{*}\right)$ associated to $\mathcal{T}^{*}$ is defined as the local-to-global map $H_{\text {cts }}^{1}\left(\mathrm{~K}_{S} / \mathrm{K}, \mathcal{T}^{*}\right) \rightarrow \prod_{v \in S} H_{\text {cts }}^{1}\left(\mathrm{~K}_{v}, \mathcal{T}^{*}\right)$. Then one readily verifies that the Pontrjagin dual of the cokernel of $\phi_{\mathcal{L}}$ is isomorphic to the quotient $\operatorname{Sel}_{\mathcal{L}^{*}}\left(\mathrm{~K}, \mathcal{T}^{*}\right) / \amalg^{1}\left(\mathrm{~K}, S, \mathcal{T}^{*}\right)$, and the Pontrjagin dual of the cokernel of $\phi_{\mathcal{L}^{*}}$ is isomorphic to $\operatorname{Sel}_{\mathcal{L}}(\mathrm{K}, \mathcal{A}) / \amalg^{1}(\mathrm{~K}, S, \mathcal{A})[\operatorname{Gr} 10$, Proposition 3.1.1]. In particular, the triviality of the dual $\operatorname{Selmer}$ group $\operatorname{Sel}_{\mathcal{L}^{*}}\left(\mathrm{~K}, \mathcal{T}^{*}\right)$ implies the validity of the hypothesis $\left(\mathrm{SUR}_{\mathcal{A}, \mathcal{L}}\right)$.

Greenberg himself has given a sufficient condition for the dual Selmer group $\operatorname{Sel}_{\mathcal{L}^{*}}\left(\mathrm{~K}, \mathcal{T}^{*}\right)$ to vanish. In order to state it, we here introduce another hypothesis:

$\left(\mathbf{C R K}_{\mathcal{A}, \mathcal{L}}\right)$ : the following equality among $\mathcal{R}$-coranks holds (recall the definition and conventions on the $\mathcal{R}$-corank in Section 3.3.1):

$$
\operatorname{corank}_{\mathcal{R}} H^{1}\left(\mathrm{~K}_{S} / \mathrm{K}, \mathcal{A}\right)=\operatorname{corank}_{\mathcal{R}} \operatorname{Sel}_{\mathcal{L}}(\mathrm{K}, \mathcal{A})+\operatorname{corank}_{\mathcal{R}} Q_{\mathcal{L}}(\mathrm{K}, \mathcal{A}) .
$$

Recall that we have the following equality on $\mathcal{R}$-coranks by the definition of the $\mathcal{L}$-Selmer group as the kernel of the global-to-local morphism (3.10):

$$
\operatorname{corank}_{\mathcal{R}} H^{1}\left(\mathrm{~K}_{S} / \mathrm{K}, \mathcal{A}\right)=\operatorname{corank}_{\mathcal{R}} \operatorname{Sel}_{\mathcal{L}}(\mathrm{K}, \mathcal{A})+\operatorname{corank}_{\mathcal{R}} Q_{\mathcal{L}}(\mathrm{K}, \mathcal{A})-\operatorname{corank}_{\mathcal{R}} \operatorname{Coker}\left(\phi_{\mathcal{L}}\right) .
$$

Hence, it is obvious that the following inequality always holds:

$$
\operatorname{corank}_{\mathcal{R}} H^{1}\left(\mathrm{~K}_{S} / \mathrm{K}, \mathcal{A}\right) \leq \operatorname{corank}_{\mathcal{R}} \operatorname{Sel}_{\mathcal{L}}(\mathrm{K}, \mathcal{A})+\operatorname{corank}_{\mathcal{R}} Q_{\mathcal{L}}(\mathrm{K}, \mathcal{A}) .
$$

It is also obvious that the condition $\left(\mathrm{CRK}_{\mathcal{A}, \mathcal{L}}\right)$ is valid if and only if the cokernel of the global-to-local morphism $\phi_{\mathcal{L}}$ is cotorsion as an $\Lambda_{0}$-module.

Proposition 3.26 ([Gr10, Proposition 3.2.1]). Suppose that $\mathcal{A}$ is a divisible $\mathcal{R}$-module and that the condition $\left(\mathrm{CRK}_{\mathcal{A}, \mathcal{L}}\right)$ holds for $\mathcal{A}$ and a specification $\mathcal{L}$. Furthermore assume that at least one of the following conditions is fulfilled:

(a) for each maximal ideal $\mathfrak{M}$ of $\mathcal{R}$, the maximal $\mathfrak{M}$-torsion submodule $\mathcal{A}[\mathfrak{M}]$ has no subquotient isomorphic to $\mu_{p}$ as a Galois representation of $\mathrm{Gal}(\overline{\mathrm{K}} / \mathrm{K})$ over $\mathbb{F}_{p}$; 
(b) the discrete module $\mathcal{A}$ is cofree as an $\Lambda_{0}$-module and, for each maximal ideal $\mathfrak{M}$ of $\mathcal{R}$, the maximal $\mathfrak{M}$-torsion submodule $\mathcal{A}[\mathfrak{M}]$ has no quotient isomorphic to $\mu_{p}$ as a Galois representation of $\mathrm{Gal}(\overline{\mathrm{K}} / \mathrm{K})$ over $\mathbb{F}_{p}$;

(c) there exists a place $v_{0}$ contained in $S$ such that $H_{\mathrm{cts}}^{0}\left(\mathrm{~K}_{v_{0}}, \mathcal{T}^{*}\right)$ is trivial and that $Q_{\mathcal{L}}\left(\mathrm{K}_{v_{0}}, \mathcal{A}\right)$ is divisible as an $\Lambda_{0}$-module.

Then the dual Selmer group $\operatorname{Sel}_{\mathcal{L}^{*}}\left(\mathrm{~K}, \mathcal{T}^{*}\right)$ is trivial; in particular, the surjectivity condition $\left(\mathrm{SUR}_{\mathcal{A}, \mathcal{L}}\right)$ holds for $\mathcal{A}$ and $\mathcal{L}$.

3.4. Inductive specialisation of the characteristic ideals. In the rest of this section we shall state the main result of the algebraic side of this article (Theorem 3.27) and prove it. As in Section 3.1.2, we denote by $F^{+}$a totally real number field of degree $d$ which satisfies the condition $\left(\mathrm{unr}_{F^{+}}\right)$. Let $f$ be a $p$-ordinary $p$-stabilised newform of cohomological weight $\kappa$, level $\mathfrak{N}$ and nebentypus $\underline{\varepsilon}$ defined on $F^{+}$, and suppose that $f$ has complex multiplication. We denote by $\eta$ the größencharacter of type $\left(A_{0}\right)$ defined on a totally imaginary quadratic extension $F$ over $F^{+}$satisfying the ordinarity condition $\left(\operatorname{ord}_{F / F^{+}}\right)$, to which the cuspform $f=\vartheta(\eta)$ is associated. As we have already mentioned, it is always possible to assume that $\eta$ is ordinary with respect to an appropriate $p$-ordinary CM type $\Sigma$ of $F$. We choose a branch character $\psi$ associated to $\eta$ and fix it (see Definition 3.8).

Recall the $\psi$-branch $\mathcal{L}_{p}^{\Sigma}(\psi)$ of Katz, Hida and Tilouine's $p$-adic $L$-function $\mathcal{L}_{p, \Sigma}^{\mathrm{KHT}}(F)$ defined in Introduction; namely $\mathcal{L}_{p}^{\Sigma}(\psi)$ is the image of $\mathcal{L}_{p, \Sigma}^{\mathrm{KHT}}(F)$ under the $\psi$-twisting map

$$
\widehat{\mathcal{O}}^{\mathrm{ur}}\left[\left[\operatorname{Gal}\left(F_{\mathfrak{C} p \infty} / F\right)\right]\right] \rightarrow \widehat{\mathcal{O}}^{\mathrm{ur}}\left[\left[\operatorname{Gal}\left(\widetilde{F}_{\infty} / F\right)\right]\right] ;\left.g \mapsto \psi(g) g\right|_{\widetilde{F}_{\infty}} .
$$

Theorem 3.27. Let the notation be as above. Furthermore assume the following three conditions;

- the nontriviality condition $(\mathbf{n t r})_{\mathfrak{P}}$ for every place $\mathfrak{P}$ of $F$ contained in $\Sigma_{p}^{c}$;

- $\left(\mathbf{I M C}_{F, \psi}\right)$ the $\left(\left(d+1+\delta_{F, p}\right)\right.$-variable $)$ Ottawa's main conjecture

$$
\operatorname{Char}_{\Lambda_{\mathcal{O}}^{\mathrm{CM}}}\left(X_{\Sigma_{p},(\psi)}\right)=\left(\mathcal{L}_{p}^{\Sigma}(\psi)\right)
$$

holds as an equality of ideals in $\Lambda_{\mathcal{O}}^{\mathrm{CM}} \hat{\otimes}_{\mathcal{O}} \widehat{\mathcal{O}}^{\mathrm{ur}}$ for the $C M$ number field $F$ and the branch character $\psi$;

- $\left(\mathbf{N V}_{\mathcal{L}_{p}^{\text {cyc }}(\vartheta(\eta))}\right)$ the cyclotomic p-adic L-function $\mathcal{L}_{p}^{\text {cyc }}(\vartheta(\eta))$ does not vanish in the sense that each component of $\mathcal{L}_{p}^{\text {cyc }}(\vartheta(\eta))$ in the indecomposable decomposition of $\Lambda_{\mathcal{O}}^{\text {cyc }} \hat{\otimes}_{\mathcal{O}} \widehat{\mathcal{O}}^{\text {ur }}$ does not equal zero.

Then we have the following equality of ideals of $\Lambda_{\mathcal{O}}^{\text {cyc }}$

$$
\left(\operatorname{Char}_{\Lambda_{\mathcal{O}}^{\mathrm{CM}}}\left(\operatorname{Sel}_{\mathcal{A}_{\eta}^{\mathrm{CM}}}^{\Sigma}\right)^{\vee}\right) \otimes_{\Lambda_{\mathcal{O}}^{\mathrm{CM}}} \Lambda_{\mathcal{O}}^{\text {cyc }}=\operatorname{Char}_{\Lambda_{\mathcal{O}}^{\text {cyc }}}\left(\operatorname{Sel}_{\mathcal{A}_{\vartheta(\eta)}^{\text {cyc }}}\right)^{\vee}
$$

where the tensor product in the left hand side is taken with respect to the canonical quotient $\operatorname{map} \Lambda_{\mathcal{O}}^{\mathrm{CM}} \rightarrow \Lambda_{\mathcal{O}}^{\mathrm{cyc}}$.

The rest of this section is devoted to the proof of Theorem 3.27. We shall verify Theorem 3.27 by induction on the Krull dimension of the coefficient ring $\Lambda_{\mathcal{O}}^{\text {cyc }}$, applying repeatedly the specialisation lemma introduced below in Section 3.4.1 (Lemma 3.29), Greenberg's criterion for almost divisibility (Theorem 3.24) and the exact control theorem (Theorem 3.18).

Remark 3.28. The conditions $\left(\mathrm{IMC}_{F, \psi}\right)$ and $\left(\mathrm{NV}_{\mathcal{L}_{p}^{\text {cyc }}(\vartheta(\eta))}\right)$ imply an important algebraic property of the Selmer group:

$$
\text { - }\left(\mathbf{C O T}_{\mathcal{A}_{\vartheta(\eta)}^{\text {cyc }}}\right) \text { the Selmer group } \operatorname{Sel}_{\mathcal{A}_{\vartheta(\eta)}^{\text {cyc }}} \text { is a cotorsion } \Lambda_{\mathcal{O}}^{\text {cyc }} \text {-module. }
$$


In order to deduce the conclusion of Theorem 3.27, we may replace the analytic condition $\left(\mathrm{NV}_{\mathcal{L}_{p}^{\text {cyc }}(\vartheta(\eta))}\right)$ by the algebraic condition $\left(\mathrm{COT}_{\mathcal{A}_{\vartheta(\eta)}^{\text {cyc }}}\right)$. We also remark that we use the analytic condition $\left(\mathrm{NV}_{\mathcal{L}_{p}^{\text {cyc }}(\vartheta(\eta))}\right)$ only at the final step of our inductive argument (see Section 3.4.6).

3.4.1. The specialisation lemma. Firstly, we recall the following elementary lemma (which we shall refer as the specialisation lemma later), describing the behaviour of characteristic ideals under specialisation procedures.

Lemma 3.29. Let $\mathcal{R}$ be a finite $\Lambda_{0}$-algebra which is isomorphic to the direct product of finitely many copies of $\Lambda_{0}$, and let $M$ be an $\mathcal{R}$-module which is finitely generated and torsion. Assume that $M$ contains no nontrivial pseudonull $\Lambda_{0}$-submodules. Let $(\Pi)$ denote a prime ideal of height one of $\Lambda_{0}$ and assume that $(\Pi)$ does not divide the characteristic ideal $\operatorname{Char}_{\mathcal{R}}(M)$ of $M$. Then the quotient module $M / \Pi M$ is finitely generated and torsion as an $\mathcal{R} / \Pi \mathcal{R}$-module, and the basechange $\operatorname{Char}_{\mathcal{R}}(M) \otimes_{\mathcal{R}} \mathcal{R} / \Pi \mathcal{R}$ of the characteristic ideal $\operatorname{Char}_{\mathcal{R}}(M)$ coincides with the characteristic ideal $\operatorname{Char}_{\mathcal{R} / \Pi \mathcal{R}}(M / \Pi M)$ of the $\mathcal{R} / \Pi \mathcal{R}$-module $M / \Pi M$ as an ideal of the quotient ring $\mathcal{R} / \Pi \mathcal{R}$.

Here we remark that (commutative) noetherian regular local rings are unique factorisation domains, and hence every height-one prime ideal of $\Lambda_{0}$ is a principal ideal.

We readily verify the lemma above in essentially the same way as the proof of Och05, Lemma 3.1]. We thus omit the proof here, just emphasising that the triviality of the pseudonull $\Lambda_{0}$-submodule of $M$ plays a crucial role in the verification of Lemma 3.29.

3.4.2. Settings on regular sequences. We now apply the results of Sections 3.3 and 3.4 to the case where $\mathcal{R}$ is the semilocal Iwasawa algebra $\Lambda_{\mathcal{O}}^{\mathrm{CM}}=\mathcal{O}\left[\left[\operatorname{Gal}\left(\widetilde{F}_{\infty} / F\right)\right]\right]$ and $\Lambda_{0}$ is the local Iwasawa algebra $\mathcal{O}[[\operatorname{Gal}(\widetilde{F} / F)]]$, which is isomorphic to the ring of formal power series in $\left(d+1+\delta_{F, p}\right)$-variables. Here we henceforth choose and fix a splitting of the Group extension

$$
1 \longrightarrow \operatorname{Gal}\left(\widetilde{F}_{\infty} / \widetilde{F}\right) \cong \operatorname{Gal}\left(F\left(\mu_{p}\right) / F\right) \longrightarrow \operatorname{Gal}\left(\widetilde{F}_{\infty} / F\right) \longrightarrow \operatorname{Gal}(\widetilde{F} / F) \longrightarrow 1
$$

and regard $\mathcal{O}[[\operatorname{Gal}(\widetilde{F} / F)]]$ as a subring of $\Lambda_{\mathcal{O}}^{\mathrm{CM}}$ by using this splitting. This identification endows the semilocal algebra $\Lambda_{\mathcal{O}}^{\mathrm{CM}}$ with the $\Lambda_{0}$-module structure. In the following arguments, we inductively find elements $\gamma_{1}, \ldots, \gamma_{d+\delta_{F, p}}$ of $\operatorname{Gal}(\widetilde{F} / F)$ so that $\gamma_{1}-1, \ldots, \gamma_{d+\delta_{F, p}}-1$ is a regular sequence of $\Lambda_{0}=\mathcal{O}[[\operatorname{Gal}(\widetilde{F} / F)]]$ contained in $\mathfrak{A}^{\text {cyc }}$ and they satisfy certain "nice" properties. We here prepare notation on regular sequences. Let $j$ be a natural number with $1 \leq j \leq d+\delta_{F, p}$ and suppose that we have already chosen elements $\gamma_{1}, \ldots, \gamma_{j}$ of $\operatorname{Gal}(\widetilde{F} / F)$ such that $\gamma_{1}-1, \ldots, \gamma_{j}-1$ is a regular sequence of $\Lambda_{0}$ contained in $\mathfrak{A}^{\text {cyc }}$. We set $x_{k}=\gamma_{k}-1$ for each $k$ with $1 \leq k \leq j$ and let $\mathfrak{A}_{j}$ denote the ideal of $\Lambda_{\mathcal{O}}^{\mathrm{CM}}$ generated by $x_{1}, \ldots, x_{j}$ with the convention that $\mathfrak{A}_{0}$ denotes the zero ideal in $\Lambda_{\mathcal{O}}^{\mathrm{CM}}$. Then the notation introduced here is compatible with that introduced in Section 3.2. We also define $\Lambda_{0}^{(j)}$ to be the quotient ring $\Lambda_{0} /\left(x_{1}, x_{2}, \ldots, x_{j}\right)$, which is a regular local ring isomorphic to the ring of formal power series in $\left(d+1+\delta_{F, p}-j\right)$-variables.

Now we introduce the local condition $\mathcal{L}_{\text {str }}$ corresponding to the strict Selmer group; namely we set for each nonarchimedean place 
$v$ in $S$

$$
L_{\mathrm{str}}\left(F_{v}, \mathcal{A}_{\eta}^{\mathrm{CM}}\left[\mathfrak{A}_{j}\right]\right)= \begin{cases}H_{\mathrm{ur}}^{1}\left(F_{v}, \mathcal{A}_{\eta}^{\mathrm{CM}}\left[\mathfrak{A}_{j}\right]\right) & \text { for } v \text { in } S \backslash\left(\Sigma_{p} \cup \Sigma_{p}^{c}\right), \\ H^{1}\left(F_{v}, \mathcal{A}_{\eta}^{\mathrm{CM}}\left[\mathfrak{A}_{j}\right]\right) & \text { for } v \in \Sigma_{p}, \\ 0 & \text { for } v \in \Sigma_{p}^{c},\end{cases}
$$

where $H_{\mathrm{ur}}^{1}\left(F_{\lambda}, \mathcal{A}_{\eta}^{\mathrm{CM}}\left[\mathfrak{A}_{j}\right]\right)$ denotes the unramified cohomology group which is defined to be $H^{1}\left(D_{\lambda} / I_{\lambda}, \mathcal{A}_{\eta}^{\mathrm{CM}}\left[\mathfrak{A}_{j}\right]^{I_{\lambda}}\right)$.

We also introduce the local condition $L_{\mathrm{str}}\left(F, \mathcal{A}_{\psi}^{\mathrm{CM}}\right)$ for the discrete $\Lambda_{\mathcal{O}}^{\mathrm{CM}}$-module $\mathcal{A}_{\psi}^{\mathrm{CM}}$ in the same manner.

The discrete $\Lambda_{\mathcal{O}}^{\mathrm{CM}} / \mathfrak{A}_{j}$-module $\mathcal{A}_{\eta}^{\mathrm{CM}}\left[\mathfrak{A}_{j}\right]$ corresponds to the continuous Galois representation $\mathcal{T}_{\eta}^{\mathrm{CM}} / \mathfrak{A}_{j} \mathcal{T}_{\eta}^{\mathrm{CM}}$; that is, the Pontrjagin duality induces an isomorphism of $\Lambda_{\mathcal{O}}^{\mathrm{CM}} / \mathfrak{A}_{j}$-linear $\operatorname{Gal}\left(F_{S} / F\right)$-representations

$$
\mathcal{A}_{\eta}^{\mathrm{CM}}\left[\mathfrak{A}_{j}\right] \cong\left(\mathcal{T}_{\eta}^{\mathrm{CM}} / \mathfrak{A}_{j} \mathcal{T}_{\eta}^{\mathrm{CM}}\right) \otimes_{\Lambda_{\mathcal{O}}^{\mathrm{CM}} / \mathfrak{A}_{j}}\left(\Lambda_{\mathcal{O}}^{\mathrm{CM}} / \mathfrak{A}_{j}\right)^{\vee} .
$$

We thus denote the Kummer dual of $\mathcal{A}_{\eta}^{\mathrm{CM}}\left[\mathfrak{A}_{j}\right]$ by $\left(\mathcal{T}_{\eta}^{\mathrm{CM}} / \mathfrak{A}_{j} \mathcal{T}_{\eta}^{\mathrm{CM}}\right)^{*}$, following Greenberg's notation introduced in Section 3.3.4. Then the local condition $\mathcal{L}_{\mathrm{str}}^{*}$ for the dual strict Selmer group $\operatorname{Sel}_{\mathcal{L}_{\text {str }}^{*}}\left(F,\left(\mathcal{T}_{\eta}^{\mathrm{CM}} / \mathfrak{A}_{j} \mathcal{T}_{\eta}^{\mathrm{CM}}\right)^{*}\right)$ is calculated as

$$
L_{\mathrm{str}}^{*}\left(F_{v},\left(\mathcal{T}_{\eta}^{\mathrm{CM}} / \mathfrak{A}_{j} \mathcal{T}_{\eta}^{\mathrm{CM}}\right)^{*}\right)= \begin{cases}H_{\mathrm{ur}}^{1}\left(F_{v},\left(\mathcal{T}_{\eta}^{\mathrm{CM}} / \mathfrak{A}_{j} \mathcal{T}_{\eta}^{\mathrm{CM}}\right)^{*}\right) & \text { for } v \in S \backslash\left(\Sigma_{p} \cup \Sigma_{p}^{c}\right), \\ 0 & \text { for } v \in \Sigma_{p}, \\ H_{\mathrm{cts}}^{1}\left(F_{v},\left(\mathcal{T}_{\eta}^{\mathrm{CM}} / \mathfrak{A}_{j} \mathcal{T}_{\eta}^{\mathrm{CM}}\right)^{*}\right) & \text { for } v \in \Sigma_{p}^{c}\end{cases}
$$

where the unramified cohomology $H_{\mathrm{ur}}^{1}\left(F_{\lambda},\left(\mathcal{T}_{\eta}^{\mathrm{CM}} / \mathfrak{A}_{j} \mathcal{T}_{\eta}^{\mathrm{CM}}\right)^{*}\right)$ is defined in the usual manner as $H_{\text {cts }}^{1}\left(D_{\lambda} / I_{\lambda},\left(\mathcal{T}_{\eta}^{\mathrm{CM}} / \mathfrak{A}_{j} \mathcal{T}_{\eta}^{\mathrm{CM}}\right)^{*, I_{\lambda}}\right)$. Indeed (3.13) follows for places of $F$ lying above $p$ directly from the definition of the local condition of the dual Selmer groups based upon the local Tate duality (3.11). Now we temporary abbreviate $\mathcal{A}_{\eta}^{\mathrm{CM}}\left[\mathfrak{A}_{j}\right]$ as $\mathcal{A}$ and $\left(\mathcal{T}_{\eta}^{\mathrm{CM}} / \mathfrak{A}_{j} \mathcal{T}_{\eta}^{\mathrm{CM}}\right)^{*}$ as $\mathcal{T}^{*}$ for brevity. Then for a place of $F$ contained in $S$ but not lying above $p$, it is well known that the unramified cohomology groups are the orthogonal complements of each other under the local Tate pairing (3.11) for finite Galois modules $\mathcal{A}\left[\mathfrak{J}^{n}\right]$ and $\mathcal{T}^{*} / \mathfrak{J}^{n} \mathcal{T}^{*}$. Here $\mathfrak{J}$ is the Jacobian radical of $\Lambda_{\mathcal{O}}^{\mathrm{CM}}$ and $n$ is an arbitrary natural number. Since $\mathcal{T}^{*}$ is obviously complete with respect to the $\mathfrak{J}$-adic topology, we readily obtain (3.13) for such a place by employing standard limit arguments based upon Tate's theorem (refer to [NSW00, Corollary 2.3.5] and [Gr06, Remark 3.5.1] for example).

3.4.3. Preliminary step: verification of several hypotheses. As the preliminary step for our inductive arguments, we verify the local hypotheses $\left(\operatorname{LOC}_{\mathcal{A}_{\eta}^{\mathrm{CM}}\left[\mathfrak{A}_{j}\right], v}^{(1)}\right),\left(\operatorname{LOC}_{\mathcal{A}_{\eta}^{\mathrm{CM}}\left[\mathfrak{A}_{j}\right], v}^{(2)}\right)$ and the almost $\Lambda_{0}^{(j)}$-divisibility of $L_{\mathrm{str}}\left(F, \mathcal{A}_{\eta}^{\mathrm{CM}}\left[\mathfrak{A}_{j}\right]\right)$ for every $j$. Firstly, we readily observe that the local hypothesis $\left(\operatorname{LOC}_{\mathcal{A}_{\eta}^{\mathrm{CM}}\left[\mathfrak{A}_{j}\right], v}^{(1)}\right.$ ) is valid for every nonarchimedean place $v$ in $S$ (and hence the local hypothesis $\left(\operatorname{LOC}_{\mathcal{A}_{\eta}^{C M}\left[\mathfrak{A}_{j}\right], v}^{(2)}\right.$ ) is automatically satisfied for every place $v$ in $S$ ). In fact the Kummer dual of $\mathcal{A}_{\eta}^{\mathrm{CM}}\left[\mathfrak{A}_{j}\right]$ is a free $\Lambda_{\mathcal{O}}^{\mathrm{CM}} / \mathfrak{A}_{j}$-module of rank one on which every element $g$ of $\operatorname{Gal}\left(F_{S} / F\right)$ acts by the multiplication of $\left.\chi_{p, \text { cyc }}^{-1} \eta^{\text {gal }}\left(g^{-1}\right) g^{-1}\right|_{\widetilde{F}_{\infty}}$. Now let $v$ be a nonarchimedean place $v$ in $S$. Since every nonarchimedean place of $F$ does not split completely in the cyclotomic $\mathbb{Z}_{p}$-extension $F_{\infty}^{\text {cyc }}$ of $F$, the image of the decomposition subgroup $D_{v}$ in $\operatorname{Gal}\left(F_{\infty}^{\text {cyc }} / F\right)$ is not trivial. In particular there exists an element $g_{0}$ of $D_{v}$ such that the image of $\xi_{g_{0}}:=\chi_{p, \text { cyc }}^{-1} \eta^{\text {gal }}\left(g_{0}^{-1}\right) g_{0}^{-1}-1$ in $\Lambda_{\mathcal{O}}^{\mathrm{CM}} / \mathfrak{A}_{j}$ does not vanish (because it is nonzero in the quotient $\Lambda_{\mathcal{O}}^{\text {cyc }}$ of $\left.\Lambda_{\mathcal{O}}^{\mathrm{CM}} / \mathfrak{A}_{j}\right)$. The definition of $\xi_{g_{0}}$ implies that, under 
the indecomposable decomposition of $\Lambda_{\mathcal{O}}^{\mathrm{CM}}$, no components of $\xi_{0}$ equal zero. Since every indecomposable component of $\Lambda_{\mathcal{O}}^{\mathrm{CM}} / \mathfrak{A}_{j}$ is a domain, we readily see that the $\xi_{0}$-torsion submodule of the free $\Lambda_{\mathcal{O}}^{\mathrm{CM}}$-module $\mathcal{A}_{\eta}^{\mathrm{CM}}\left[\mathfrak{A}_{j}\right]$ is trivial. This implies that the $D_{v}$-invariant of the Kummer dual of $\mathcal{A}_{\eta}^{\mathrm{CM}}\left[\mathfrak{A}_{j}\right]$ equals zero, and thus $\left(\operatorname{LOC}_{\mathcal{A}_{\eta}^{\mathrm{CM}}\left[\mathfrak{H}_{j}\right], v}^{(1)}\right)$ holds for every $j$ and for every nonarchimedean $v$ in $S$.

We now verify the almost divisibility of the local condition $L_{\mathrm{str}}\left(F_{v}, \mathcal{A}_{\eta}^{\mathrm{CM}}\left[\mathfrak{A}_{j}\right]\right)$. There is nothing to prove for a place in $\Sigma_{p}^{c}$ since the local condition is trivial at such a place. For a place $\mathfrak{P}$ in $\Sigma_{p}$, the local condition $L_{\mathrm{str}}\left(F_{\mathfrak{P}}, \mathcal{A}_{\eta}^{\mathrm{CM}}\left[\mathfrak{A}_{j}\right]\right)$ coincides with the whole cohomology group $H^{1}\left(F_{\mathfrak{P}}, \mathcal{A}_{\eta}^{\mathrm{CM}}\left[\mathfrak{A}_{j}\right]\right)$, and its almost $\Lambda_{0}^{(j)}$-divisibility follows from the hypothesis $\left(\mathrm{LOC}_{\mathcal{A}_{\eta}^{\mathrm{CM}\left[\mathfrak{A}_{j}\right], \mathfrak{P}}}^{(2)}\right)$ and [Gr06, Proposition 5.4]. Finally take a place $\lambda$ from $S \backslash\left(\Sigma_{p} \cup \Sigma_{p}^{c}\right)$. By the same argument as the proof of Theorem 3.18, the image of the inertia subgroup $I_{\lambda}$ under the Galois character $\eta^{\text {gal }}$ is finite and contained in the set of roots of unity in $\mathcal{O}$. Thus $\mathcal{A}_{\eta}^{\mathrm{CM}}\left[\mathfrak{A}_{j}\right]^{I_{\lambda}}$ is isomorphic to $\mathcal{A}_{\eta}^{\mathrm{CM}}\left[\mathfrak{A}_{j}, \varpi^{n} \Lambda_{\mathcal{O}}^{\mathrm{CM}}\right]$ for some nonnegative integer $n$, which is almost $\Lambda_{0}^{(j)}$-divisible (observe that $\mathcal{A}_{\eta}^{\mathrm{CM}}\left[\mathfrak{A}_{j}, \varpi^{n} \Lambda_{\mathcal{O}}^{\mathrm{CM}}\right]$ is divisible for every heightone prime ideal of $\Lambda_{j}^{(0)}$ relatively prime to $\left.\varpi \Lambda_{0}^{(j)}\right)$. Since $H_{\mathrm{ur}}^{1}\left(F_{\lambda}, \mathcal{A}_{\eta}^{\mathrm{CM}}\left[\mathfrak{A}_{j}\right]\right)$ is isomorphic to a quotient of $\mathcal{A}_{\eta}^{\mathrm{CM}}\left[\mathfrak{A}_{j}\right]^{I_{\lambda}}, H_{\mathrm{ur}}^{1}\left(F_{\lambda}, \mathcal{A}_{\eta}^{\mathrm{CM}}\left[\mathfrak{A}_{j}\right]\right)$ must also be almost $\Lambda$-divisible.

Remark 3.30. It is also possible to verify the hypotheses $\left(\mathrm{LOC}_{\mathcal{A}_{\psi}^{\mathrm{CM}}, v}^{(1)}\right)$ and $\left(\mathrm{LOC}_{\mathcal{A}_{\psi}^{\mathrm{CM}}, v}^{(2)}\right)$ for each nonarchimedean place contained in $S$ and the almost $\Lambda_{0}$-divisibility of the local condition $L_{\mathrm{str}}\left(F, \mathcal{A}_{\psi}^{\mathrm{CM}}\right)$ by exactly the same arguments as above.

3.4.4. First step: almost divisibility of the initial strict Selmer group. We shall verify the almost $\Lambda_{0}$-divisibility of the initial strict Selmer group $\operatorname{Sel}_{\mathcal{A}_{\eta}^{\text {CM }}}^{\Sigma \text { str }}$. Identifying $\operatorname{Sel}_{\mathcal{A}_{\eta}^{\text {CMM }}}^{\Sigma \text { str }}$ with $\mathrm{Tw}_{\eta^{\mathrm{gal},-1} \psi}\left(\mathrm{Sel}_{\mathcal{A}_{\psi}^{\mathrm{CM}}}^{\Sigma, \text { str }}\right)$ by exactly the same arguments as in Remark 3.17, we readily see that the almost $\Lambda_{0}$-divisibility of $\mathrm{Sel}_{\mathcal{A}_{\eta}^{\mathrm{CM}}}^{\Sigma, \mathrm{str}}$ is equivalent to that of $\mathrm{Sel}_{\mathcal{A}_{\psi}^{\mathrm{CM}}}^{\Sigma, \mathrm{str}}$. Concerning the verification of the almost $\Lambda_{0}$-divisibility of $\operatorname{Sel}_{\mathcal{A}_{\psi}^{\mathrm{CM}}}^{\Sigma \text {,str }}$, we first observe that an arbitrary place $\mathfrak{P}$ in $\Sigma_{p}$ satisfies the extra condition (c) proposed in Proposition 3.26, It is obvious because $Q_{\mathcal{L}_{\text {str }}}\left(F_{\mathfrak{P}}, \mathcal{A}_{\psi}^{\mathrm{CM}}\right)$ is trivial for each $\mathfrak{P}$ in $\Sigma_{p}$. Thus, in order to deduce the almost $\Lambda_{0}$-divisibility of $\mathrm{Sel}_{\mathcal{A}_{\eta}^{\mathrm{CM}}}^{\Sigma \text { str }}$ from Theorem 3.24 and Proposition 3.26 , it suffices to verify the remaining two hypotheses $\left(\mathrm{CRK}_{\mathcal{A}_{\psi}^{\mathrm{CM}}, \mathcal{L}_{\text {str }}}\right)$ and $\left(\mathrm{LEO}_{\mathcal{A}_{\psi}^{\mathrm{CM}}}\right)$; recall that we have already verified in the preceding step (Section 3.4.3) all the other hypotheses required in Theorem 3.24,

We have observed in Remark 3.17 and Lemma 3.21 that the Iwasawa module $X_{\Sigma_{p},(\psi)}$ and the Pontrjagin dual of the strict Selmer group $\operatorname{Sel}_{\mathcal{A}_{\psi}}^{\Sigma \text {,str }}$ are pseudoisomorphic to each other as $\Lambda_{\mathcal{O}}^{\mathrm{CM}}$-modules.

Now we introduce the following hypothesis concerning the algebraic structure of the Iwasawa module $X_{\Sigma_{p},(\psi)}$ : $\left(\mathbf{T O R}_{X_{\Sigma_{p},(\psi)}}\right)$ : the $\Lambda_{\mathcal{O}}^{\mathrm{CM}}$-module $X_{\Sigma_{p},(\psi)}$ is a torsion module.

Note that the condition $\left(\mathrm{TOR}_{X_{\Sigma_{p},(\psi)}}\right)$ is equivalent to the following condition on the algebraic structure of the strict Selmer group $\operatorname{Sel}_{\mathcal{A}_{\psi}^{\mathrm{CM}}}^{\Sigma \text {,str }}$ by the discussion above:

$\left(\mathbf{C O T}_{\mathcal{A}_{\psi}^{\mathrm{CM}}}\right)$ : the strict Selmer group $\mathrm{Sel}_{\mathcal{A}_{\psi}^{\mathrm{CM}}}^{\Sigma, \mathrm{str}}$ is cotorsion as a $\Lambda_{\mathcal{O}}^{\mathrm{CM}}$-module. 
Lemma 3.31. The following two statements are equivalent for the discrete $\Lambda_{\mathcal{O}}^{\mathrm{CM}}$-module $\mathcal{A}_{\psi}^{\mathrm{CM}}$

(1) both the conditions $\left(\mathrm{CRK}_{\mathcal{A}_{\psi}^{\mathrm{CM}}, \mathcal{L}_{\mathrm{str}}}\right)$ and $\left(\mathrm{LEO}_{\mathcal{A}_{\psi}^{\mathrm{CM}}}\right)$ hold;

(2) the condition $\left(\mathrm{COT}_{\mathcal{A}_{\psi}^{\mathrm{CM}}}\right)$ (or, equivalently, the condition $\left.\left(\mathrm{TOR}_{X_{\Sigma_{p},(\psi)}}\right)\right)$ holds.

Proof. We abbreviate the $\Lambda_{\mathcal{O}}^{\mathrm{CM}}$-corank of a cofinitely generated $\Lambda_{\mathcal{O}}^{\mathrm{CM}}$-module $M$ just as "corank $M$ " in the proof of Lemma 3.31 so as to simplify the notation. We shall henceforth verify that the following equation among $\Lambda_{\mathcal{O}}^{\mathrm{CM}}$-coranks holds:

$$
\text { corank } \operatorname{Sel}_{\mathcal{A}_{\psi}^{\mathrm{CM}}}^{\Sigma, \mathrm{str}}=\operatorname{corank} \amalg^{2}\left(F, S, \mathcal{A}_{\psi}^{\mathrm{CM}}\right)+\operatorname{corank} \operatorname{Coker}\left(\phi_{\mathcal{L}_{\text {str }}}\right) .
$$

Once the equation (3.14) is proved, the equivalence between the assertions (1) and (2) immediately follows; for the assertion (1) is fulfilled if and only if the right hand side of the equation (3.14) equals zero, whereas the assertion (2) is fulfilled if and only if the left hand side of (3.14) equals zero (recall that the validity of the corank condition $\left(\mathrm{CRK}_{\mathcal{A}_{\psi}^{\mathrm{CM}}, \mathcal{L}_{\text {str }}}\right)$ is equivalent to the $\Lambda_{\mathcal{O}}^{\mathrm{CM}}$-cotorsionness of the cokernel of $\left.\phi_{\mathcal{L}_{\text {str }}}\right)$.

In order to deduce the equation (3.14), we consider the equation

$$
\begin{aligned}
\operatorname{corank} \operatorname{Sel}_{\mathcal{A}_{\psi}^{\mathrm{CM}}}^{\Sigma, \mathrm{str}}=\operatorname{corank} & H^{1}\left(F_{S} / F, \mathcal{A}_{\psi}^{\mathrm{CM}}\right) \\
& -\operatorname{corank} Q_{\mathcal{L}_{\text {str }}}\left(F, \mathcal{A}_{\psi}^{\mathrm{CM}}\right)+\operatorname{corank} \operatorname{Coker}\left(\phi_{\mathcal{L}_{\text {str }}}\right),
\end{aligned}
$$

which is deduced from the exact sequence

$$
0 \longrightarrow \operatorname{Sel}_{\mathcal{A}_{\psi}^{\mathrm{CM}}}^{\Sigma, \mathrm{str}} \longrightarrow H^{1}\left(F_{S} / F, \mathcal{A}_{\psi}^{\mathrm{CM}}\right) \stackrel{\phi_{\mathcal{L}_{\mathrm{str}}}}{\longrightarrow} Q_{\mathcal{L}_{\text {str }}}\left(F, \mathcal{A}_{\psi}^{\mathrm{CM}}\right) \longrightarrow \operatorname{Coker}\left(\phi_{\mathcal{L}_{\text {str }}}\right) \longrightarrow 0
$$

defining the strict Selmer group $\mathrm{Sel}_{\mathcal{A}_{\psi}^{\mathrm{CM}}}^{\Sigma, \text { str }}$, and calculate the right hand side of (3.15) by utilising Euler-Poincaré characteristic formulae studied in [Gr06].

We first calculate the $\Lambda_{\mathcal{O}}^{\mathrm{CM}}$-corank of the global cohomology group $H^{1}\left(F_{S} / F, \mathcal{A}_{\psi}^{\mathrm{CM}}\right)$. By the global Euler-Poincaré characteristic formula [Gr06, Proposition 4.1], we have the equality:

$$
\begin{aligned}
\operatorname{corank} & H^{1}\left(F_{S} / F, \mathcal{A}_{\psi}^{\mathrm{CM}}\right) \\
& =\operatorname{corank} H^{0}\left(F_{S} / F, \mathcal{A}_{\psi}^{\mathrm{CM}}\right)+\operatorname{corank} H^{2}\left(F_{S} / F, \mathcal{A}_{\psi}^{\mathrm{CM}}\right)+d \operatorname{corank}\left(\mathcal{A}_{\psi}^{\mathrm{CM}}\right) .
\end{aligned}
$$

Recall that $d$ denotes the extension degree $\left[F^{+}: \mathbb{Q}\right]$ of the totally real field $F^{+}$over $\mathbb{Q}$. We claim that $H^{0}\left(F_{S} / F, \mathcal{A}_{\psi}^{\mathrm{CM}}\right)$ is a cotorsion $\Lambda_{\mathcal{O}}^{\mathrm{CM}}$-module. Indeed $\mathcal{A}_{\psi}^{\mathrm{CM}}$ is naturally identified with the Pontrjagin dual of $\Lambda_{\mathcal{O}}^{\mathrm{CM}}$, on which an element $g$ of $\operatorname{Gal}\left(F_{S} / F\right)$ acts by the multiplication of $\left.\psi(g) g\right|_{\widetilde{F}_{\infty}}$. Let us take an arbitrary element $g_{0}$ of $\operatorname{Gal}\left(F_{S} / F\right)$ whose image in $\operatorname{Gal}(\widetilde{F} / F)$ under the natural surjection $\operatorname{Gal}\left(F_{S} / F\right) \rightarrow \operatorname{Gal}(\widetilde{F} / F)$ is nontrivial. Then we readily see that the $g_{0}$-invariant of $\mathcal{A}_{\psi}^{\mathrm{CM}}$ is isomorphic to a $\Lambda_{\mathcal{O}}^{\mathrm{CM}}$-module $\left(\Lambda_{\mathcal{O}}^{\mathrm{CM}} /\left(\left.\psi\left(g_{0}\right) g_{0}\right|_{\widetilde{F}_{\infty}}-1\right) \Lambda_{\mathcal{O}}^{\mathrm{CM}}\right)^{\vee}$, which is obviously a cotorsion $\Lambda_{\mathcal{O}}^{\mathrm{CM}}$-module. The zeroth cohomology $H^{0}\left(F_{S} / F, \mathcal{A}_{\psi}^{\mathrm{CM}}\right)$ is obviously a subgroup of the $g_{0}$-invariant of $\mathcal{A}_{\psi}^{\mathrm{CM}}$, and thus it is also a cotorsion $\Lambda_{\mathcal{O}}^{\mathrm{CM}}$ :

$$
\operatorname{corank} H^{0}\left(F_{S} / F, \mathcal{A}_{\psi}^{\mathrm{CM}}\right)=0 .
$$

We next investigate the $\Lambda_{\mathcal{O}}^{\mathrm{CM}}$-corank of the second cohomology group $H^{2}\left(F_{S} / F, \mathcal{A}_{\psi}^{\mathrm{CM}}\right)$. The nine-term exact sequence due to Poitou and Tate (see [NSW00, (8.6.3) i)] and [Gr06, 
Section 4.B]) implies that the cokernel of the global-to-local homomorphism

$$
\phi^{(2)}: H^{2}\left(F_{S} / F, \mathcal{A}_{\psi}^{\mathrm{CM}}\right) \rightarrow \prod_{v \in S \backslash \Sigma_{\infty}} H^{2}\left(F_{v}, \mathcal{A}_{\psi}^{\mathrm{CM}}\right)
$$

is isomorphic to the Pontrjagin dual of $H_{\mathrm{cts}}^{0}\left(F_{S} / F,\left(\mathcal{T}_{\psi}^{\mathrm{CM}}\right)^{*}\right)$, which is trivial because the hypothesis $\left(\mathrm{LOC}_{\mathcal{A}_{\psi}^{\mathrm{CM}}, v}^{(1)}\right.$ ) holds for every $v$ in $S \backslash \Sigma_{\infty}$. The kernel of $\phi^{(2)}$ is $\amalg^{2}\left(F, S, \mathcal{A}_{\psi}^{\mathrm{CM}}\right)$ by definition. Furthermore, by virtue of the local Tate duality, the Pontrjagin dual of $H^{2}\left(F_{v}, \mathcal{A}_{\psi}^{\mathrm{CM}}\right)$ is isomorphic to $H_{\mathrm{cts}}^{0}\left(F_{v},\left(\mathcal{T}_{\psi}^{\mathrm{CM}}\right)^{*}\right)$ for each $v$ in $S \backslash \Sigma_{\infty}$, and hence it equals zero by the hypothesis $\left(\operatorname{LOC}_{\mathcal{A}_{\psi}}^{(1)}{ }_{v}\right.$ ) again. Combining these calculations, we conclude that the corank of the whole second cohomology group $H^{2}\left(F_{S} / F, \mathcal{A}_{\psi}^{\mathrm{CM}}\right)$ equals that of the generalised second Tate-Šafarevič group $\amalg^{2}\left(F, S, \mathcal{A}_{\psi}^{\mathrm{CM}}\right)$ :

$$
\text { corank } H^{2}\left(F_{S} / F, \mathcal{A}_{\psi}^{\mathrm{CM}}\right)=\text { corank } \amalg^{2}\left(F, S, \mathcal{A}_{\psi}^{\mathrm{CM}}\right) .
$$

By (3.16), (3.17) and (3.18), we have the following formula:

$$
\text { corank } H^{1}\left(F_{S} / F, \mathcal{A}_{\psi}^{\mathrm{CM}}\right)=\operatorname{corank} \amalg^{2}\left(F, S, \mathcal{A}_{\psi}^{\mathrm{CM}}\right)+d \operatorname{corank} \mathcal{A}_{\psi}^{\mathrm{CM}} \text {. }
$$

We now study the $\Lambda_{\mathcal{O}}^{\mathrm{CM}}$-corank of $Q_{\mathcal{L}_{\text {str }}}\left(F, \mathcal{A}_{\psi}^{\mathrm{CM}}\right)$.

First, let us take a place $\mathfrak{P}^{c}$ in $\Sigma_{p}^{c}$. Applying the local Euler-Poincaré characteristic formula [Gr06, Proposition 4.2] to the local cohomology group $H^{1}\left(F_{\mathfrak{P}^{c}}, \mathcal{A}_{\psi}^{\mathrm{CM}}\right)$, we obtain

$$
\begin{aligned}
& \text { corank } Q_{\mathcal{L}_{\text {str }}}\left(F_{\mathfrak{P}^{c}}, \mathcal{A}_{\psi}^{\mathrm{CM}}\right)=\operatorname{corank} H^{1}\left(F_{\mathfrak{P}^{c}}, \mathcal{A}_{\psi}^{\mathrm{CM}}\right) \\
& =\operatorname{corank} H^{0}\left(F_{\mathfrak{P}^{c}}, \mathcal{A}_{\psi}^{\mathrm{CM}}\right)+\operatorname{corank} H^{2}\left(F_{\mathfrak{P}^{c}}, \mathcal{A}_{\psi}^{\mathrm{CM}}\right)+\left[F_{\mathfrak{P}^{c}}: \mathbb{Q}_{p}\right] \operatorname{corank} \mathcal{A}_{\psi}^{\mathrm{CM}} .
\end{aligned}
$$

We claim that the $\Lambda_{\mathcal{O}}^{\mathrm{CM}}$-coranks of $H^{0}\left(F_{\mathfrak{P}^{c}}, \mathcal{A}_{\psi}^{\mathrm{CM}}\right)$ and $H^{2}\left(F_{\mathfrak{P}^{c}}, \mathcal{A}_{\psi}^{\mathrm{CM}}\right)$ are both equal to zero. Indeed, since each place of $F$ above $p$ does not split completely in $\widetilde{F} / F$, the image of $D_{\mathfrak{P}_{c}}$ in $\operatorname{Gal}(\widetilde{F} / F)$ contains a nontrivial element. We thus apply the same arguments as those we made for $H^{0}\left(F_{S} / F, \mathcal{A}_{\psi}^{\mathrm{CM}}\right)$ and conclude that $H^{0}\left(F_{\mathfrak{P}^{c}}, \mathcal{A}_{\psi}^{\mathrm{CM}}\right)$ is cotorsion as a $\Lambda_{\mathcal{O}}^{\mathrm{CM}}$-module. On the other hand, as we have already checked in the computation of the $\Lambda_{\mathcal{O}}^{\mathrm{CM}}$-corank of $H^{2}\left(F_{S} / F, \mathcal{A}_{\psi}^{\mathrm{CM}}\right)$, the second local cohomology group $H^{2}\left(F_{\mathfrak{P}^{c}}, \mathcal{A}_{\psi}^{\mathrm{CM}}\right)$ is trivial due to the hypothesis $\left(\mathrm{LOC}_{\mathcal{A}_{\psi}^{\mathrm{CM}}, \mathfrak{P}^{c}}^{(1)}\right)$. Therefore we obtain the following formula for each place $\mathfrak{P}^{c}$ in $\Sigma_{p}^{c}$ :

$$
\operatorname{corank} Q_{\mathcal{L}_{\text {str }}}\left(F_{\mathfrak{P}^{c}}, \mathcal{A}_{\psi}^{\mathrm{CM}}\right)=\left[F_{\mathfrak{P}^{c}}: \mathbb{Q}_{p}\right] \operatorname{corank} \mathcal{A}_{\psi}^{\mathrm{CM}} .
$$

Next, let $\lambda$ be a place in $S \backslash \Sigma_{p}$. We also calculate the corank of $Q_{\mathcal{L}_{\text {str }}}\left(F_{\lambda}, \mathcal{A}_{\psi}^{\mathrm{CM}}\right)$ by applying the local Euler-Poincaré characteristics formula [Gr06, Proposition 4.2] to the local cohomology group $H^{1}\left(F_{\lambda}, \mathcal{A}_{\psi}^{\mathrm{CM}}\right)$ as follows:

$$
\begin{aligned}
& \text { corank } Q_{\mathcal{L}_{\text {str }}}\left(F_{\lambda}, \mathcal{A}_{\psi}^{\mathrm{CM}}\right) \\
& =\operatorname{corank} H^{1}\left(F_{\lambda}, \mathcal{A}_{\psi}^{\mathrm{CM}}\right)-\operatorname{corank} H_{\mathrm{ur}}^{1}\left(F_{\lambda}, \mathcal{A}_{\psi}^{\mathrm{CM}}\right) \\
& =\operatorname{corank} H^{0}\left(F_{\lambda}, \mathcal{A}_{\psi}^{\mathrm{CM}}\right)+\operatorname{corank} H^{2}\left(F_{\lambda}, \mathcal{A}_{\psi}^{\mathrm{CM}}\right)-\operatorname{corank} H_{\mathrm{ur}}^{1}\left(F_{\lambda}, \mathcal{A}_{\psi}^{\mathrm{CM}}\right) .
\end{aligned}
$$

We claim that the right hand side of this equality is zero, that is,

$$
\operatorname{corank} Q_{\mathcal{L}_{\text {str }}}\left(F_{\lambda}, \mathcal{A}_{\psi}^{\mathrm{CM}}\right)=0
$$


holds. Indeed the second cohomology group $H^{2}\left(F_{\lambda}, \mathcal{A}_{\psi}^{\mathrm{CM}}\right)$ is trivial by the hypothesis $\left(\mathrm{LOC}_{\mathcal{A}_{\psi}^{\mathrm{CM}}, \lambda}^{(1)}\right)$ as we have already mentioned, and the $\Lambda_{\mathcal{O}}^{\mathrm{CM}}$-corank of the unramified cohomology $H_{\mathrm{ur}}^{1}\left(F_{\lambda}, \mathcal{A}_{\psi}^{\mathrm{CM}}\right)$ equals that of $H^{0}\left(F_{\lambda}, \mathcal{A}_{\psi}^{\mathrm{CM}}\right)$ since the residue characteristic at $\lambda$ does not equal $p$; this fact is well known for finite Galois modules and we readily generalise it by using a specialisation trick similar to one used in the proof of [Gr06, Proposition 4.1].

Substituting (3.19), (3.20) and (3.21) for the equation (3.15), we obtain the desired equation (3.14) as

$$
\begin{aligned}
& \operatorname{corank} \operatorname{Sel}_{\mathcal{A}_{\psi}^{\mathrm{CM}}}^{\Sigma, \mathrm{str}} \\
& =\operatorname{corank} \amalg^{2}\left(F, S, \mathcal{A}_{\psi}^{\mathrm{CM}}\right)+d \operatorname{corank} \mathcal{A}_{\psi}^{\mathrm{CM}} \\
& -\sum_{\mathfrak{P}^{c} \in \Sigma_{p}^{c}}\left[F_{\mathfrak{P}^{c}}: \mathbb{Q}_{p}\right] \operatorname{corank} \mathcal{A}_{\psi}^{\mathrm{CM}}+\operatorname{corank} \operatorname{Coker}\left(\phi_{\mathcal{L}_{\text {str }}}\right) \\
& =\operatorname{corank} \amalg^{2}\left(F, S, \mathcal{A}_{\psi}^{\mathrm{CM}}\right)+\operatorname{corank} \operatorname{Coker}\left(\phi_{\mathcal{L}_{\text {str }}}\right)+\left(d-\sum_{\mathfrak{p} \mid p \mathfrak{r}_{F^{+}}}\left[F_{\mathfrak{p}}^{+}: \mathbb{Q}_{p}\right]\right) \operatorname{corank} \mathcal{A}_{\psi}^{\mathrm{CM}} \\
& =\operatorname{corank} \amalg^{2}\left(F, S, \mathcal{A}_{\psi}^{\mathrm{CM}}\right)+\operatorname{corank} \operatorname{Coker}\left(\phi_{\mathcal{L}_{\text {str }}}\right)
\end{aligned}
$$

The assumption $\left(\operatorname{TOR}_{X_{\Sigma_{p},(\psi)}}\right)$ clearly holds if the Galois group $X_{\Sigma_{p}}=\operatorname{Gal}\left(M_{\Sigma_{p}} / \widetilde{K}_{\infty}^{\mathrm{CM}}\right)$ itself is torsion as an $\mathcal{O}\left[\left[\mathrm{Gal}\left(\widetilde{K}_{\infty}^{\mathrm{CM}} / F\right)\right]\right]$-module. As a part of their research on the anticyclotomic Iwasawa main conjecture for CM number fields, Hida and Tilouine have thoroughly studied the torsionness property of $X_{\Sigma_{p}}$ in [HT94, Section 1.2], and showed that $X_{\Sigma_{p}}$ is torsion over $\mathcal{O}\left[\left[\mathrm{Gal}\left(\widetilde{K}_{\infty}^{\mathrm{CM}} / F\right)\right]\right]$ if the $\Sigma$-Leopoldt condition (the condition $\left(\mathscr{L}_{\widetilde{K}_{\infty}^{\mathrm{CM}}, \Sigma}\right)$ in the terminology of [HT94]) is valid for the extension $\widetilde{K}_{\infty}^{\mathrm{CM}} / F$. See [HT94, Theorem 1.2.2 (ii)] for details of the discussion (note that our extension $\widetilde{K}_{\infty}^{\mathrm{CM}}$ is always regarded as a subfield of the ray class field $F_{\mathfrak{C} p}$ modulo $\mathfrak{C} p^{\infty}$ over $F$ for an appropriate integral ideal $\mathfrak{C}$ of $F$ ). Since the extension $\widetilde{K}_{\infty}^{\mathrm{CM}} / F$ contains the cyclotomic $\mathbb{Z}_{p}$-extension, we observe that the $\Sigma$-Leopoldt condition is valid for the extension $\widetilde{K}_{\infty}^{\mathrm{CM}} / F$ by reducing to the validity of the weak Leopold conjecture for the cyclotomic $\mathbb{Z}_{p}$-extension of an arbitrary number field due to the classical works of Iwasawa and Greenberg [Gr76, Proof of Theorem 3] (or by directly applying [HT94, Theorem 1.2.2 (iii)]). Consequently the assumption $\left(\operatorname{TOR}_{X_{\Sigma_{p},(\psi)}}\right)$ is fulfilled and we have verified the following proposition:

Proposition 3.32. The strict Selmer group $\operatorname{Sel}_{\mathcal{A}_{\psi}^{\mathrm{CM}}}^{\Sigma, \mathrm{str}}$ of $\mathcal{A}_{\psi}^{\mathrm{CM}}$ is cotorsion over $\Lambda_{\mathcal{O}}^{\mathrm{CM}}$ and almost divisible over $\Lambda_{0}$. The same claim holds for the strict Selmer group $\operatorname{Sel}_{\mathcal{A}_{\eta}^{\mathrm{CM}}}^{\Sigma \text { of }}$ of $\mathcal{A}_{\eta}^{\mathrm{CM}}$.

As a corollary of our computation so far, we deduce a consequence on the algebraic structure of the Iwasawa module $X_{\Sigma_{p},(\psi)}$ when the Pontrjagin dual of $\mathrm{Sel}_{\mathcal{A}_{\psi}^{\mathrm{CM}}}^{\Sigma, \mathrm{str}}$ is exactly isomorphic to $X_{\Sigma_{p},(\psi)}$. Namely,

Corollary 3.33 (Theorem (C). Assume that the nontriviality condition $(\mathrm{ntr})_{\psi, \mathfrak{F}^{c}}$ is valid for each place $\mathfrak{P}^{c}$ of $F$ belonging to $\Sigma_{p}^{c}$ and that the order of the branch character $\psi$ is relatively prime to $p$ (in particular $\psi$ is not trivial). Then the Iwasawa module $X_{\Sigma_{p},(\psi)}$ does not contain nontrivial pseudonull $\Lambda_{0}$-submodules. 
Proof. Under the assumptions, the Pontrjagin dual of $\mathrm{Sel}_{\mathcal{A}_{\psi}^{\mathrm{CM}}}^{\mathrm{L} \text {,str }}$ is exactly isomorphic to $X_{\Sigma_{p},(\psi)}$ by Remark 3.9 and Lemma 3.21, The statement then follows from Proposition 3.32 ,

Remark 3.34. The triviality of pseudonull submodules of the Iwasawa module $X_{\Sigma_{p},(\psi)}$ has been already studied by Perrin-Riou in [PR81, Théorème 2.4] only when $F$ is an imaginary quadratic field and $\psi$ is a größencharacter associated to an elliptic curve with complex multiplication. Her method essentially utilises Wintenberger's structure theorem on projective limits of local unit groups Win80] combined with Greenberg's classical result on the triviality of the pseudonull submodules contained in the " $p$-ramified Iwasawa module" Gr78, Proposition 5], which is rather different from ours. It was not clear to us if the method used in [PR81] could be extended to general CM number fields or not.

3.4.5. Intermediate steps: inductive specialisation of the Selmer group. Next we shall inductively specialise the strict Selmer group $\mathrm{Sel}_{\mathcal{A}_{\eta}^{\mathrm{CM}}}^{\Sigma \text { str }}$ so that the characteristic ideal of its Pontrjagin dual behaves compatibly with respect to each specialisation procedure.

We use the same notation as in Section 3.4.2, in particular $\mathfrak{A}_{j}$ denotes the ideal of $\Lambda_{\mathcal{O}}^{\mathrm{CM}}$ generated by a regular sequence $x_{1}, \ldots, x_{j}$ with $x_{k}=\gamma_{k}-1$. We also recall that the ideal $\mathfrak{A}^{\text {cyc }}$ of $\Lambda_{\mathcal{O}}^{\mathrm{CM}}$ is defined as the kernel of the natural surjection $\Lambda_{\mathcal{O}}^{\mathrm{CM}} \rightarrow \Lambda_{\mathcal{O}}^{\text {cyc }}$. Now let us consider the following three conditions $(\Gamma 0)_{j},(\Gamma 1)_{j}$ and $(\Gamma 2)_{j}$ on the fixed elements $\gamma_{1}, \ldots, \gamma_{j}$ of $\operatorname{Gal}(\widetilde{F} / F)$ :

$(\Gamma 0)_{j}$ the sequence $x_{1}, \ldots, x_{j}$ is a regular sequence of $\Lambda_{\mathcal{O}}^{\mathrm{CM}}$ contained in $\mathfrak{A}^{\text {cyc}}$;

$(\Gamma 1)_{j}$ the hypothesis $\left(\operatorname{LEO}_{\mathcal{A}_{\eta}^{\mathrm{CM}}\left[\mathfrak{A}_{j}\right]}\right)$ is fulfilled for $\mathcal{A}_{\eta}^{\mathrm{CM}}\left[\mathfrak{A}_{j}\right]$;

$(\Gamma 2)_{j}$ the dual Selmer group $\operatorname{Sel}_{\mathcal{L}_{\text {str }}^{*}}\left(F,\left(\mathcal{T}_{\eta}^{\mathrm{CM}} / \mathfrak{A}_{j} \mathcal{T}_{\eta}^{\mathrm{CM}}\right)^{*}\right)$ is trivial.

Here we regard the condition $(\Gamma 0)_{0}$ as the empty condition. The following proposition is the key of our specialisation arguments.

Proposition 3.35. Let the notation be as above and assume that the condition (ntr) $\mathfrak{P}^{c}$ is fulfilled for every place $\mathfrak{P}^{c}$ of $F$ contained in $\Sigma_{p}^{c}$. Suppose that, for a natural number $j$ with $1 \leq j \leq d+\delta_{F, p}-1$, all the conditions $(\Gamma 0)_{j-1},(\Gamma 1)_{j-1}$ and $(\Gamma 2)_{j-1}$ are fulfilled on a set of elements $\gamma_{1}, \ldots, \gamma_{j-1}$ of $\operatorname{Gal}\left(\widetilde{F} / F_{\infty}^{\mathrm{cyc}}\right)$. Let $\mathrm{Sel}_{\mathcal{A}_{\eta}^{\mathrm{CM}}\left[\mathfrak{A}_{j-1}\right]}^{\mathrm{s}, \mathrm{st}}$ be the strict Selmer group of $\mathcal{A}_{\eta}^{\mathrm{CM}}\left[\mathfrak{A}_{j-1}\right]$ and $\left(\mathrm{Sel}_{\mathcal{A}_{\eta}^{\mathrm{CM}}\left[\mathfrak{A}_{j-1}\right]}^{\Sigma, \mathrm{str}}\right) \vee$ its Pontrjagin dual. Then there exists an element $\gamma_{j}$ of $\operatorname{Gal}\left(\widetilde{F} / F_{\infty}^{\mathrm{cyc}}\right)$ such that the element $x_{j}=\gamma_{j}-1$ of $\Lambda_{\mathcal{O}}^{\mathrm{CM}}$ does not divide in $\Lambda_{\mathcal{O}}^{\mathrm{CM}} / \mathfrak{A}_{j-1}$ the characteristic ideal of the torsion part of $\left(\mathrm{Sel}_{\mathcal{A}_{\eta}^{\mathrm{CM}}\left[\mathfrak{A}_{j-1}\right]}^{\Sigma, \mathrm{str}}\right)^{\vee}$, and all the conditions $(\Gamma 0)_{j},(\Gamma 1)_{j}$ and $(\Gamma 2)_{j}$ are fulfilled on the tuples $\gamma_{1}, \ldots, \gamma_{j-1}$ and $\gamma_{j}$.

We postpone the proof of Proposition 3.35 until the end of this paragraph, and we here deduce the basechange compatibility of the characteristic ideal of $\left(\mathrm{Sel}_{\mathcal{A}_{\eta}^{\mathrm{CM}}}^{\Sigma, \mathrm{str}}\right)^{\vee}$ under intermediate specialisation procedures from Proposition 3.35. Firstly we note that, when $j$ equals zero, all the conditions $(\Gamma 0)_{0},(\Gamma 1)_{0}$ and $(\Gamma 2)_{0}$ are fulfilled; indeed the condition $(\Gamma 0)_{0}$ is empty by convention, and the condition $(\Gamma 1)_{0}$ follows from Lemma 3.31 and the cotorsionness of the strict Selmer group $\mathrm{Sel}_{\mathcal{A}_{\eta}^{\text {CM }}}^{\Sigma \text {,str }}($ refer to the argument in Section 3.4.4). The triviality of the dual Selmer group $\operatorname{Sel}_{\mathcal{L}_{\text {str }}^{*}}\left(F, \mathcal{T}_{\eta}^{*}\right)$, namely the condition $(\Gamma 2)_{0}$, also follows from Lemma 3.31 and the cotorsionness of the strict Selmer group $\operatorname{Sel}_{\mathcal{A}_{\eta}^{\mathrm{CM}}}^{\Sigma, \text { str }}$, combined with Proposition 3.26, observe that an arbitrary place $\mathfrak{P}^{c}$ contained in $\Sigma_{p}^{c}$ satisfies the extra condition (c) proposed in Proposition 3.26. 
Now let $j$ be a natural number with $1 \leq j \leq d+\delta_{F, p}-1$, and assume that we have already chosen elements $\gamma_{1}, \ldots, \gamma_{j-1}$ of $\operatorname{Gal}\left(\widetilde{F} / F_{\infty}^{\text {cyc }}\right)$ so that all the conditions $(\Gamma 0)_{j-1},(\Gamma 1)_{j-1}$ and $(\Gamma 2)_{j-1}$ are fulfilled for them. As an induction hypothesis, we further assume that $\mathrm{Sel}_{\mathcal{A}_{\eta}^{\mathrm{CM}}\left[\mathfrak{A}_{j-1}\right]}^{\Sigma, \mathrm{str}}$ is cotorsion as a $\Lambda_{\mathcal{O}}^{\mathrm{CM}} / \mathfrak{A}_{j-1}$-module. Theorem 3.24 then enables us to conclude that the strict Selmer group $\operatorname{Sel}_{\mathcal{A}_{\eta}^{\mathrm{CM}}\left[\mathfrak{A}_{j-1}\right]}^{\Sigma, \mathrm{str}}$ is almost $\Lambda_{0}^{(j-1)}$-divisible. Let $\left(\operatorname{Sel}_{\mathcal{A}_{\eta}^{\mathrm{CM}}\left[\mathfrak{A}_{j-1}\right]}^{\Sigma, \mathrm{str}}\right)^{\vee}$ denote the Pontrjagin dual of the strict Selmer group $\operatorname{Sel}_{\mathcal{A}_{\eta}^{\mathrm{CM}}\left[\mathfrak{A}_{j-1}\right]}^{\Sigma \mathrm{str}}$. We apply Proposition 3.35 and find an element $\gamma_{j}$ of $\operatorname{Gal}\left(\widetilde{F} / F_{\infty}^{\text {cyc }}\right)$ so that $x_{j}=\gamma_{j}-1$ does not divide the characteristic ideal of $\left(\mathrm{Sel}_{\mathcal{A}_{\eta}^{\mathrm{MM}}\left[\mathfrak{A}_{j-1}\right]}^{\Sigma \text { str }}\right)^{\vee}$ and all the conditions $(\Gamma 0)_{j},(\Gamma 1)_{j}$ and $(\Gamma 2)_{j}$ are fulfilled for the tuples $\gamma_{1}, \ldots, \gamma_{j-1}$ and $\gamma_{j}$. Then, from the Exact Control Theorem 3.18 and the Specialisation Lemma 3.29, we readily deduce the cotorsionness of the strict Selmer group $\mathrm{Sel}_{\mathcal{A}_{\eta}^{\mathrm{CM}}\left[\mathfrak{A}_{j}\right]}^{\mathrm{Cstr}}$ as a $\Lambda_{\mathcal{O}}^{\mathrm{CM}} / \mathfrak{A}_{j}$-module, and obtain the equality

$$
\left(\operatorname{Char}_{\Lambda_{\mathcal{O}}^{\mathrm{CM}} / \mathfrak{A}_{j-1}}\left(\operatorname{Sel}_{\mathcal{A}_{\eta}^{\mathrm{CM}\left[\mathfrak{A}_{j-1}\right]}}^{\Sigma, \mathrm{str}}\right)^{\vee}\right) \otimes_{\Lambda_{\mathcal{O}}^{\mathrm{CM}} / \mathfrak{A}_{j-1}} \Lambda_{\mathcal{O}}^{\mathrm{CM}} / \mathfrak{A}_{j}=\operatorname{Char}_{\Lambda_{\mathcal{O}}^{\mathrm{CM}} / \mathfrak{A}_{j}}\left(\operatorname{Sel}_{\mathcal{A}_{\eta}^{\mathrm{CM}}\left[\mathfrak{A}_{j}\right]}^{\Sigma, \operatorname{str}}\right)^{\vee}
$$

The induction proceeds until $j$ achieves $d+\delta_{F, p}-1$, and consequently we see that the strict Selmer group $\operatorname{Sel}_{\mathcal{A}_{\eta}^{\mathrm{CM}}\left[\mathfrak{A}_{d+\delta_{F, p}-1}, \mathrm{str}\right.}$ is cotorsion as a $\Lambda_{\mathcal{O}}^{\mathrm{CM}} / \mathfrak{A}_{d+\delta_{F, p}-1}$-module and the following equality among ideals of $\Lambda_{\mathcal{O}}^{\mathrm{CM}} / \mathfrak{A}_{d+\delta_{F, p}-1}$ holds:

$$
\begin{aligned}
\left(\operatorname{Char}_{\Lambda_{\mathcal{O}}^{\mathrm{GM}}}\left(\operatorname{Sel}_{\mathcal{A}_{\eta}^{\mathrm{CMM}}}^{\Sigma, \mathrm{str}}\right)^{\vee}\right) \otimes_{\Lambda_{\mathcal{O}}^{\mathrm{CM}}} \Lambda_{\mathcal{O}}^{\mathrm{CM}} / \mathfrak{A}_{d+\delta_{F, p}-1} \\
=\operatorname{Char}_{\Lambda_{\mathcal{O}}^{\mathrm{CM}} / \mathfrak{A}_{d+\delta_{F, p}-1}}\left(\operatorname{Sel}_{\mathcal{A}_{\eta}^{\mathrm{Cs}}\left[\mathfrak{A}_{d+\delta_{F, p}-1}\right]}\right)^{\vee} .
\end{aligned}
$$

Remark 3.36. We warn that we here exclude the case where $j$ equals $d+\delta_{F, p}$ due to the constraint imposed on $j$ in Proposition 3.35, the case where $j$ equals $d+\delta_{F, p}$ shall be dealt with later in Section 3.4.6 as the final specialisation procedure.

Let us return to the proof of Proposition 3.35. We first observe that the condition $(\Gamma 2)_{j}$ - the triviality of the dual Selmer group - is automatically fulfilled for an appropriate choice of $\gamma_{j}$.

Proposition 3.37. Let $j$ be as above and let $\gamma_{1}, \ldots, \gamma_{j-1}$ be elements of $\operatorname{Gal}\left(\widetilde{F} / F_{\infty}^{\text {cyc }}\right)$ for which all the conditions $(\Gamma 0)_{j-1},(\Gamma 1)_{j-1}$ and $(\Gamma 2)_{j-1}$ stated before Proposition 3.35 are fulfilled. Assume further that the condition $(\mathrm{ntr})_{\mathfrak{P}^{c}}$ is fulfilled for every place $\mathfrak{P}^{c}$ of $F$ contained in $\Sigma_{p}^{c}$. Let $\gamma_{j}$ be an element of $\operatorname{Gal}\left(\widetilde{F} / F_{\infty}^{\text {cyc }}\right)$ such that the tuples $\gamma_{1}, \ldots, \gamma_{j-1}, \gamma_{j}$ satisfy the condition $(\Gamma 0)_{j}$ and that $x_{j}=\gamma_{j}-1$ does not divide the characteristic ideal of the torsion part of $\left(\mathrm{Sel}_{\mathcal{A}_{\eta}^{\mathrm{CM}}\left[\mathfrak{A}_{j-1}\right]}^{\mathrm{S}, \mathrm{str}}\right)^{\vee}$ in $\Lambda_{\mathcal{O}}^{\mathrm{CM}} / \mathfrak{A}_{j-1}$. Then the dual strict Selmer group $\operatorname{Sel}_{\mathcal{L}_{\text {str }}^{*}}\left(F,\left(\mathcal{T}_{\eta}^{\mathrm{CM}} / \mathfrak{A}_{j} \mathcal{T}_{\eta}^{\mathrm{CM}}\right)^{*}\right)$ of $\left(\mathcal{T}_{\eta}^{\mathrm{CM}} / \mathfrak{A}_{j} \mathcal{T}_{\eta}^{\mathrm{CM}}\right)^{*}$ is trivial.

Let us verify Proposition 3.35 admitting Proposition 3.37.

Proof of Proposition 3.35. Let $\gamma_{1}, \ldots, \gamma_{j-1}$ be elements of $\operatorname{Gal}\left(\widetilde{F} / F_{\infty}^{\text {cyc }}\right)$ as in the statement of Proposition 3.35 and $H$ the closed subgroup of $\operatorname{Gal}\left(\widetilde{F} / F_{\infty}^{\text {cyc }}\right)$ topologically generated by $\gamma_{1}, \ldots, \gamma_{j-1}$. Define $\mathscr{C}_{H}$ as the set of all the elements of $\bar{\Gamma}_{j-1}:=\operatorname{Gal}\left(\widetilde{F} / F_{\infty}^{\text {cyc }}\right) / H$ with nontrivial images in $\bar{\Gamma}_{j-1} /\left(\bar{\Gamma}_{j-1}\right)^{p}$. Let us denote by $Y_{j-1}^{(1)}$ the finite set of height-one prime 
ideals in $\Lambda_{0}^{(j-1)}$ dividing the characteristic ideal of the torsion part of $\left(\operatorname{Sel}_{\mathcal{A}_{\eta}^{\mathrm{CM}}\left[\mathfrak{A}_{j-1}\right]}^{\Sigma, \mathrm{str}}\right)^{\vee}$, which is obviously a finite set. Meanwhile, as we have remarked in Section 3.3 .2 , the validity of the hypothesis $\left(\mathrm{LEO}_{\mathcal{A}_{\eta}^{\mathrm{CM}\left[\mathfrak{A}_{j-1}\right]}}\right)$ implies the existence of a finite set $Y_{j-1}^{(2)}$ of exceptional prime ideals of height one in $\Lambda_{0}^{(j-1)}$ in the sense that the hypothesis $\left(\operatorname{LEO}_{\mathcal{A}_{\eta}^{\mathrm{CM}}\left[\mathfrak{A}_{j-1}\right][\bar{\Pi}]}\right)$ is true for every height-one prime ideal $(\bar{\Pi})$ of $\Lambda_{0}^{(j-1)}$ which is not contained in $Y_{j-1}^{(2)}$. Since the set of principal ideals in $\Lambda_{0}^{(j-1)}$ defined as $\left\{(\bar{\gamma}-1) \Lambda_{0}^{(j-1)} \mid \bar{\gamma} \in \mathscr{C}_{H}\right\}$ is infinite, we can choose an element $\bar{\gamma}_{j}$ of $\mathscr{C}_{H}$ such that the prime ideal $\left(\bar{\gamma}_{j}-1\right)$ in $\Lambda_{0}^{(j-1)}$ generated by $\bar{\gamma}_{j}-1$ is contained in neither $Y_{j-1}^{(1)}$ nor $Y_{j-1}^{(2)}$. Let us take an arbitrary lift $\gamma_{j}$ of $\bar{\gamma}_{j}$ to $\operatorname{Gal}\left(\widetilde{F} / F_{\infty}^{\mathrm{cyc}}\right)$. By construction, both the conditions $(\Gamma 0)_{j}$ and $(\Gamma 1)_{j}$ are fulfilled on the tuples $\gamma_{1}, \ldots, \gamma_{j-1}, \gamma_{j}$ of $\operatorname{Gal}\left(\widetilde{F} / F_{\infty}^{\text {cyc }}\right)$. We now complete the proof of Proposition 3.35 since the condition $(\Gamma 2)_{j}$ is also fulfilled on them by virtue of Proposition 3.37 .

The remaining issue is the verification of Proposition 3.37. Let us abbreviate in the proof of Proposition 3.37 the continuous $\operatorname{Gal}\left(F_{S} / F\right)$-representation $\left(\mathcal{T}_{\eta}^{\mathrm{CM}} / \mathfrak{A}_{k} \mathcal{T}_{\eta}^{\mathrm{CM}}\right)^{*}$ and the discrete $\operatorname{Gal}\left(F_{S} / F\right)$-representation $\mathcal{A}_{\eta}^{\mathrm{CM}}\left[\mathfrak{A}_{k}\right]$ as $\mathcal{T}_{\eta,(k)}^{*}$ and $\mathcal{A}_{\eta,(k)}$ respectively where $k$ equals $j-1$ or $j$. We also use the abbreviation $\amalg_{\mathcal{A}_{\eta,(k)}}^{1}$ for the $S$-fine Selmer group $\amalg^{1}\left(F, S, \mathcal{A}_{\eta,(k)}\right)$ of $\mathcal{A}_{\eta,(k)}$. Note that $\mathcal{T}_{\eta,(k)}^{*}$ is a free $\Lambda_{\mathcal{O}}^{\mathrm{CM}} / \mathfrak{A}_{k}$-module of rank one on which every element $g$ of $\operatorname{Gal}\left(F_{S} / F\right)$ acts by the multiplication of $\left.\chi_{p, \text { cyc }}^{-1} \eta^{\text {gal }}\left(g^{-1}\right) g\right|_{\widetilde{F}}$. First recall that the cokernel of the global-to-local morphism

$$
\phi_{\mathcal{L}_{\text {str }}^{*,(k)}}: H^{1}\left(F_{S} / F, \mathcal{T}_{\eta,(k)}^{*}\right) \rightarrow Q_{\mathcal{L}_{\text {str }}^{*,(k)}}\left(F, \mathcal{T}_{\eta,(k)}^{*}\right)
$$

is isomorphic to the kernel of the natural surjection $\left(\operatorname{Sel}_{\mathcal{A}_{\eta,(k)}}^{\Sigma \text {,str }}\right)^{\vee} \rightarrow\left(\amalg_{\mathcal{A}_{\eta,(k)}}^{1}\right)^{\vee}$ for every $k$ (see Section 3.3.4 for details); in particular, there exists a short exact sequence

$$
0 \longrightarrow \operatorname{Coker}\left(\phi_{\mathcal{L}_{\text {str }}^{*,(k)}}\right) \longrightarrow\left(\operatorname{Sel}_{\mathcal{A}_{\eta,(k)}^{\Sigma, \operatorname{str}}}\right)^{\vee} \longrightarrow\left(\amalg_{\mathcal{A}_{\eta,(k)}}^{1}\right)^{\vee} \longrightarrow 0
$$

for $k=j-1$ or $j$. We consider the commutative diagram

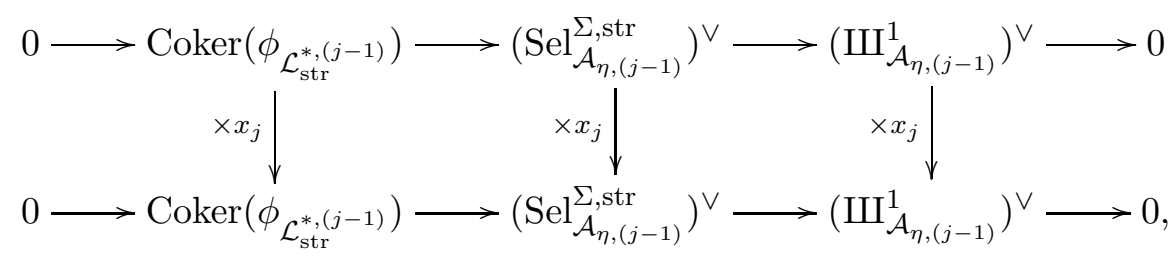

whose rows are the exact sequences (3.23) for $k=j-1$ and vertical maps are multiplication by $x_{j}$. Note that the Pontrjagin dual of the strict Selmer group $\operatorname{Sel}_{\mathcal{A}_{\eta,(j-1)}}^{\Sigma, \text { str }}$ does not contain nontrivial pseudonull $\Lambda_{0}^{(j-1)}$-submodules due to the assumptions $(\Gamma 0)_{j-1},(\Gamma 1)_{j-1}$ and $(\Gamma 2)_{j-1}$ combined with Theorem 3.24. Since $x_{j}$ does not divide the characteristic ideal of $\left(\operatorname{Sel}_{\mathcal{A}_{\eta,(j-1)}}^{\Sigma, \text { str }}\right)^{\vee}$ by assumption, the triviality of the pseudonull submodules of $\left(\operatorname{Sel}_{\mathcal{A}_{\eta,(j-1)}}^{\Sigma, \text { str }}\right)^{\vee}$ implies that the middle vertical arrow of (3.24) is injective (and so is the left vertical arrow). Thus, applying the snake lemma to the diagram (3.24), we obtain a four-term exact sequence

$$
0 \longrightarrow\left(\amalg_{\mathcal{A}_{\eta,(j-1)}}^{1}\right)^{\vee}\left[x_{j}\right] \stackrel{\tilde{\delta}_{1}^{\vee}}{\longrightarrow} \mathcal{C}^{(j-1)} \longrightarrow\left(\operatorname{Sel}_{\mathcal{A}_{\eta,(j-1)}^{\Sigma, \operatorname{str}}}\left[x_{j}\right]\right)^{\vee} \longrightarrow\left(\amalg_{\mathcal{A}_{\eta,(j-1)}}^{1}\left[x_{j}\right]\right)^{\vee} \longrightarrow 0
$$


where we denote by $\tilde{\delta}_{1}^{\vee}$ the connecting homomorphism and define $\mathcal{C}^{(j-1)}$ as follows:

$$
\mathcal{C}^{(j-1)}:=\operatorname{Coker}\left[\operatorname{Coker}\left(\phi_{\mathcal{L}_{\text {str }}^{*,(j-1)}}\right) \stackrel{\times x_{j}}{\longrightarrow} \operatorname{Coker}\left(\phi_{\mathcal{L}_{\text {str }}^{*,(j-1)}}\right)\right] .
$$

We also remark that there exists a natural commutative diagram

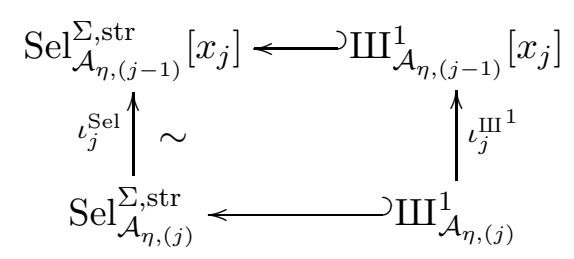

both of whose vertical morphisms $\iota_{j}^{\text {Sel }}$ and $\iota_{j}^{\amalg^{1}}$ are induced from the natural inclusion $\iota_{j}^{\mathcal{A}}: \mathcal{A}_{\eta,(j)}=\mathcal{A}_{\eta,(j-1)}\left[x_{j}\right] \hookrightarrow \mathcal{A}_{\eta,(j-1)}$ of $\operatorname{Gal}\left(F_{S} / F\right)$-modules. Note that the left vertical map $\iota_{j}^{\text {Sel }}$ is an isomorphism due to the Exact Control Theorem 3.18. Combining the Pontrjagin dual of the commutative diagram (3.27) with the exact sequence (3.25), we obtain the following diagram with exact rows:

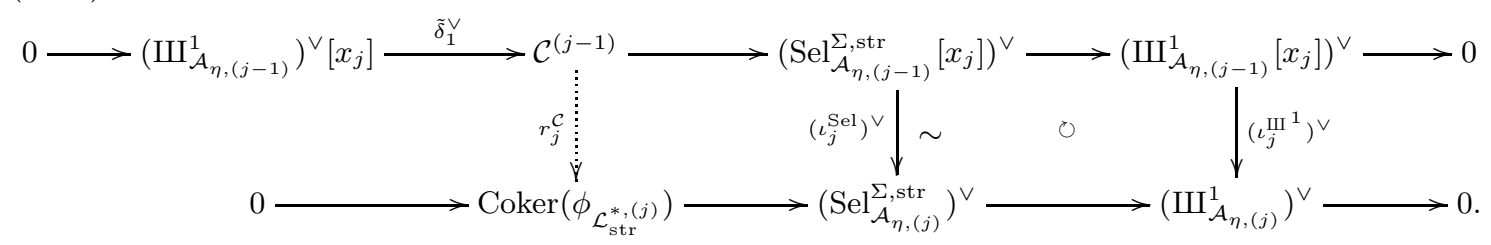

Thanks to the commutativity of (3.27), a homomorphism $r_{j}^{\mathcal{C}}: \mathcal{C}^{(j-1)} \rightarrow \operatorname{Coker}\left(\phi_{\mathcal{L}_{\text {str }}^{*,(j)}}\right)($ the dotted vertical arrow in the diagram (3.28) ) is induced. An easy diagram chase on (3.28) enables us to verify that the kernel of the induced homomorphism $r_{j}^{\mathcal{C}}$ coincides with the image of $\tilde{\delta}_{1}^{\vee}$, and therefore we obtain an exact sequence

$$
0 \longrightarrow\left(\amalg_{\mathcal{A}_{\eta,(j-1)}}^{1}\right)^{\vee}\left[x_{j}\right] \stackrel{\tilde{\delta}_{1}^{\vee}}{\longrightarrow} \mathcal{C}^{(j-1)} \longrightarrow \operatorname{Coker}\left(\phi_{\mathcal{L}_{\mathrm{str}}^{*(j)}}\right) .
$$

Next recall that the dual Selmer group of $\mathcal{T}_{\eta,(k)}^{*}$, which we denote by $\operatorname{Sel}_{\mathcal{T}_{\eta,(k)}^{*}}^{* \text { str }}$ for brevity, is defined in terms of the exact sequence

$$
0 \longrightarrow \operatorname{Sel}_{\mathcal{T}_{\eta,(k)}^{*, \mathrm{str}}}^{*} \longrightarrow H_{\mathrm{cts}}^{1}\left(F_{S} / F, \mathcal{T}_{\eta,(k)}^{*}\right) \stackrel{\phi_{\mathcal{L}_{\mathrm{str}}^{*,(k)}}^{\longrightarrow}}{\longrightarrow} Q_{\mathcal{L}_{\mathrm{str}}^{*,(k)}}\left(F, \mathcal{T}_{\eta,(k)}^{*}\right) \longrightarrow \operatorname{Coker}\left(\phi_{\mathcal{L}_{\mathrm{str}}^{*,(k)}}^{*(k)}\right) \longrightarrow 0
$$

for $k=j-1$ or $j$. When $k$ equals $j-1$, the triviality assumption $(\Gamma 2)_{j-1}$ on $\operatorname{Sel}_{\mathcal{T}_{\eta,(j-1)}^{*}}^{* \operatorname{str}}$ suggests that the global-to-local morphism $\phi_{\mathcal{L}_{\text {str }}^{*,(j-1)}}$ in (3.30) is injective. We thus consider the commutative diagram

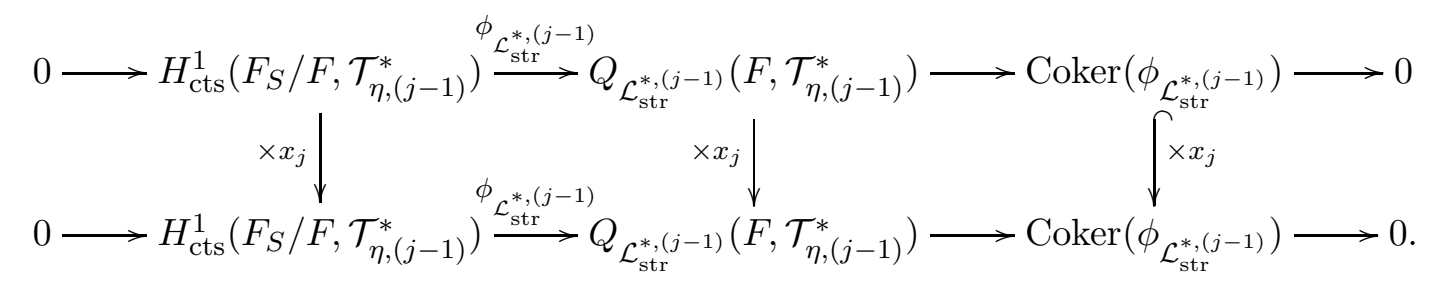


whose horizontal rows are short exact sequences obtained by (3.30) for $k=j-1$ and all of whose vertical maps are multiplication by $x_{j}$. Note that the right vertical arrow of (3.31) is injective since it is the same as the left vertical arrow of (3.24). The snake lemma applied to the diagram (3.31) then suggests that the cokernels of its vertical morphisms form a short exact sequence

$$
0 \longrightarrow H_{(j-1)}^{1, *} / x_{j} H_{(j-1)}^{1, *} \longrightarrow Q_{(j-1)}^{*} / x_{j} Q_{(j-1)}^{*} \longrightarrow \mathcal{C}^{(j-1)} \longrightarrow 0
$$

with abbreviated notation

$$
H_{(j)}^{i, *}:=H_{\mathrm{cts}}^{i}\left(F_{S} / F, \mathcal{T}_{\eta,(j)}^{*}\right), \quad Q_{(j-1)}^{*}:=Q_{\mathcal{L}_{\mathrm{str}}^{*,(j-1)}}\left(F, \mathcal{T}_{\eta,(j-1)}^{*}\right) .
$$

Next we shall relate the short exact sequence (3.32) with the sequence (3.30) for $k=j$, which concerns the dual Selmer group $\operatorname{Sel}_{\mathcal{T}_{\eta,(j)}^{*}}^{* \text { str }}$. To this end, we first observe that the short exact sequence

$$
0 \longrightarrow \mathcal{T}_{\eta,(j-1)}^{*} \stackrel{\times x_{j}}{\longrightarrow} \mathcal{T}_{\eta,(j-1)}^{*} \stackrel{\pi_{j}^{\mathcal{T}}}{\longrightarrow} \mathcal{T}_{\eta,(j)}^{*} \longrightarrow 0
$$

of $\operatorname{Gal}\left(F_{S} / F\right)$-modules induces the following exact sequence:

$$
0 \longrightarrow H_{(j-1)}^{1, *} / x_{j} H_{(j-1)}^{1, *} \stackrel{\pi_{j}^{H}}{\longrightarrow} H_{(j)}^{1, *} \stackrel{\delta_{(j)}^{1}}{\longrightarrow} H_{(j-1)}^{2, *}\left[x_{j}\right] \longrightarrow 0 .
$$

We shall construct a homomorphism connecting $Q_{(j-1)}^{*} / x_{j} Q_{(j-1)}^{*}$ with $Q_{(j)}^{*}$ in a way similar to the construction of $\pi_{j}^{H}$. Let $v$ be a place of $F$ contained in $S$ and let us denote by $\pi_{j, v}^{H}$ the injection on the local cohomology groups

$$
\pi_{j, v}^{H}: H_{\mathrm{cts}}^{1}\left(F_{v}, \mathcal{T}_{\eta,(j-1)}^{*}\right) / x_{j} H_{\mathrm{cts}}^{1}\left(F_{v}, \mathcal{T}_{\eta,(j-1)}^{*}\right) \rightarrow H_{\mathrm{cts}}^{1}\left(F_{v}, \mathcal{T}_{\eta,(j)}^{*}\right)
$$

induced by the cohomological long exact sequence associated to the sequence (3.34).

Lemma 3.38. For each $v$ in $S$, the map $\pi_{j, v}^{H}$ induces an injection

$$
\pi_{j, v}^{Q}: Q_{\mathcal{L}_{\mathrm{str}}^{*,(j-1)}}\left(F_{v}, \mathcal{T}_{\eta,(j-1)}^{*}\right) / x_{j} Q_{\mathcal{L}_{\mathrm{str}}^{*,(j-1)}}\left(F_{v}, \mathcal{T}_{\eta,(j-1)}^{*}\right) \longrightarrow Q_{\mathcal{L}_{\mathrm{str}}^{*,(j)}}\left(F_{v}, \mathcal{T}_{\eta,(j)}^{*}\right)
$$

with a cokernel isomorphic to $H_{\mathrm{cts}}^{2}\left(F_{v}, \mathcal{T}_{\eta,(j-1)}^{*}\right)\left[x_{j}\right]$.

Proof. In this proof, $k$ denotes either $j-1$ or $j$. There is nothing to prove for a place $\mathfrak{P}^{c}$ in $\Sigma_{p}^{c}$ since the local quotient $Q_{\mathcal{L}_{\text {str }}^{*,(k)}}\left(F_{\mathfrak{P}^{c}}, \mathcal{T}_{\eta,(k)}^{*}\right)$ itself is trivial. For a place $\mathfrak{P}$ in $\Sigma_{p}$, the local quotient $Q_{\mathcal{L}_{\mathrm{str}}^{*,(k)}}\left(F_{\mathfrak{P}}, \mathcal{T}_{\eta,(k)}^{*}\right)$ coincides with the whole local cohomology group $H_{\text {cts }}^{1}\left(F_{\mathfrak{P}}, \mathcal{T}_{\eta,(k)}^{*}\right)$ by definition, and thus we define the desired injection $\pi_{j, \mathfrak{P}}^{Q}$ to be $\pi_{j, \mathfrak{P}}^{H}$ itself. Now let us consider a place $\lambda$ in $S$ which does not divide $p$. If the inertia subgroup $I_{\lambda}$ acts nontrivially on $\mathcal{T}_{\eta,(k)}^{*}$, or in other words, if $\eta^{\text {gal }}$ is ramified at $\lambda$, the $I_{\lambda}$-invariant submodule of $\mathcal{T}_{\eta,(k)}^{*}$ is trivial; it is because every element $g$ of $I_{\lambda}$ acts as multiplication of the nontrivial element $\eta^{\text {gal }}\left(g^{-1}\right)$ on each component of the rank-one free $\Lambda_{\mathcal{O}}^{\mathrm{CM}} / \mathfrak{A}_{k}$-module $\mathcal{T}_{\eta,(k)}^{*}$, which is torsionfree as a $\Lambda_{0}^{(k)}$-module. This observation implies the triviality of the unramified cohomology group $H_{\mathrm{ur}}^{1}\left(F_{\lambda}, \mathcal{T}_{\eta,(k)}^{*}\right)$, and thus the desired map $\pi_{j, \lambda}^{Q}$ should be defined as $\pi_{j, \lambda}^{H}$. Finally assume that $I_{\lambda}$ acts trivially on $\mathcal{T}_{\eta,(k)}^{*}$, or in other words, assume that $\eta^{\text {gal }}$ is unramified at $\lambda$. Then the unramified cohomology group $H_{\mathrm{ur}}^{1}\left(F_{\lambda}, \mathcal{T}_{\eta,(k)}^{*}\right)$ is just the first 
continuous cohomology group $H_{\text {cts }}^{1}\left(D_{\lambda} / I_{\lambda}, \mathcal{T}_{\eta,(k)}^{*}\right)$ of the procyclic group $D_{\lambda} / I_{\lambda}$, and thus the surjection $\pi_{j}^{\mathcal{T}}: \mathcal{T}_{\eta,(j-1)}^{*} \rightarrow \mathcal{T}_{\eta,(j)}^{*}$ induces an injection

$$
\pi_{j, \lambda}^{\mathrm{ur}}: H_{\mathrm{ur}}^{1}\left(F_{\lambda}, \mathcal{T}_{\eta,(j-1)}^{*}\right) / x_{j} H_{\mathrm{ur}}^{1}\left(F_{\lambda}, \mathcal{T}_{\eta,(j-1)}^{*}\right) \longrightarrow H_{\mathrm{ur}}^{1}\left(F_{\lambda}, \mathcal{T}_{\eta,(j)}^{*}\right)
$$

with a cokernel isomorphic to $H_{\mathrm{cts}}^{2}\left(D_{\lambda} / I_{\lambda}, \mathcal{T}_{\eta,(j-1)}^{*}\right)\left[x_{j}\right]$ due to the cohomological long exact sequence associated to (3.34) regarded as the short exact sequences of continuous $D_{\lambda} / I_{\lambda}$-modules. However the procyclic group $D_{\lambda} / I_{\lambda} \cong \hat{\mathbb{Z}}$ has cohomological dimension one, and hence the the cokernel of $\pi_{j, \lambda}^{\mathrm{ur}}$ should vanish; in other words the injection $\pi_{j, v}^{\mathrm{ur}}$ is indeed an isomorphism. We therefore obtain a commutative diagram with exact rows

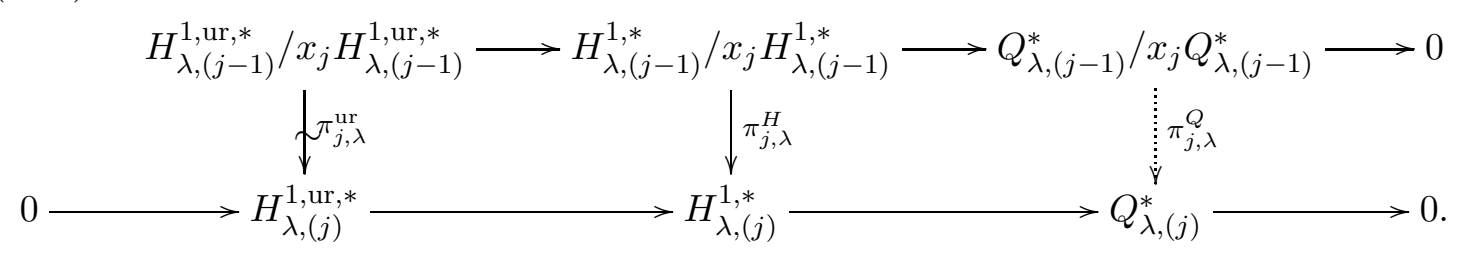

Here $H_{\mathrm{cts}}^{1}\left(F_{\lambda}, \mathcal{T}_{\eta,(k)}^{*}\right), H_{\mathrm{cts}}^{1}\left(D_{\lambda} / I_{\lambda}, \mathcal{T}_{\eta,(k)}^{*}\right)$ and $Q_{\mathcal{L}_{\mathrm{str}}^{*,(k)}}\left(F_{\lambda}, \mathcal{T}_{\eta,(k)}^{*}\right)$ are abbreviated as $H_{\lambda,(k)}^{1, *}$, $H_{\lambda,(k)}^{1, \mathrm{ur}, *}$ and $Q_{\lambda,(k)}^{*}$ respectively. The commutativity of the left square in (3.36) is due to the functoriality of the inflation map $H_{\lambda,(k)}^{1, \text { ur,* }} \rightarrow H_{\lambda,(k)}^{1, *}$, by virtue of which $\pi_{j, \lambda}^{H}$ induces a map $\pi_{j, \lambda}^{Q}$ on the quotient modules (the dotted arrow in (3.36) ). Moreover the cokernel of $\pi_{j, \lambda}^{Q}$ is isomorphic to that of $\pi_{j, \lambda}^{H}$ due to the isomorphy of the left vertical map $\pi_{j, \lambda}^{\mathrm{ur}}$. This is the end of the proof since the cokernel of $\pi_{j, \lambda}^{H}$ is isomorphic to $H^{2}\left(F_{\lambda}, \mathcal{T}_{\eta,(j-1)}^{*}\right)\left[x_{j}\right]$ due to the cohomological long exact sequence associated to (3.34).

Set $\pi_{j}^{Q}$ as $\pi_{j}^{Q}=\left(\pi_{j, v}^{Q}\right)_{v \in S}$ and consider the diagram

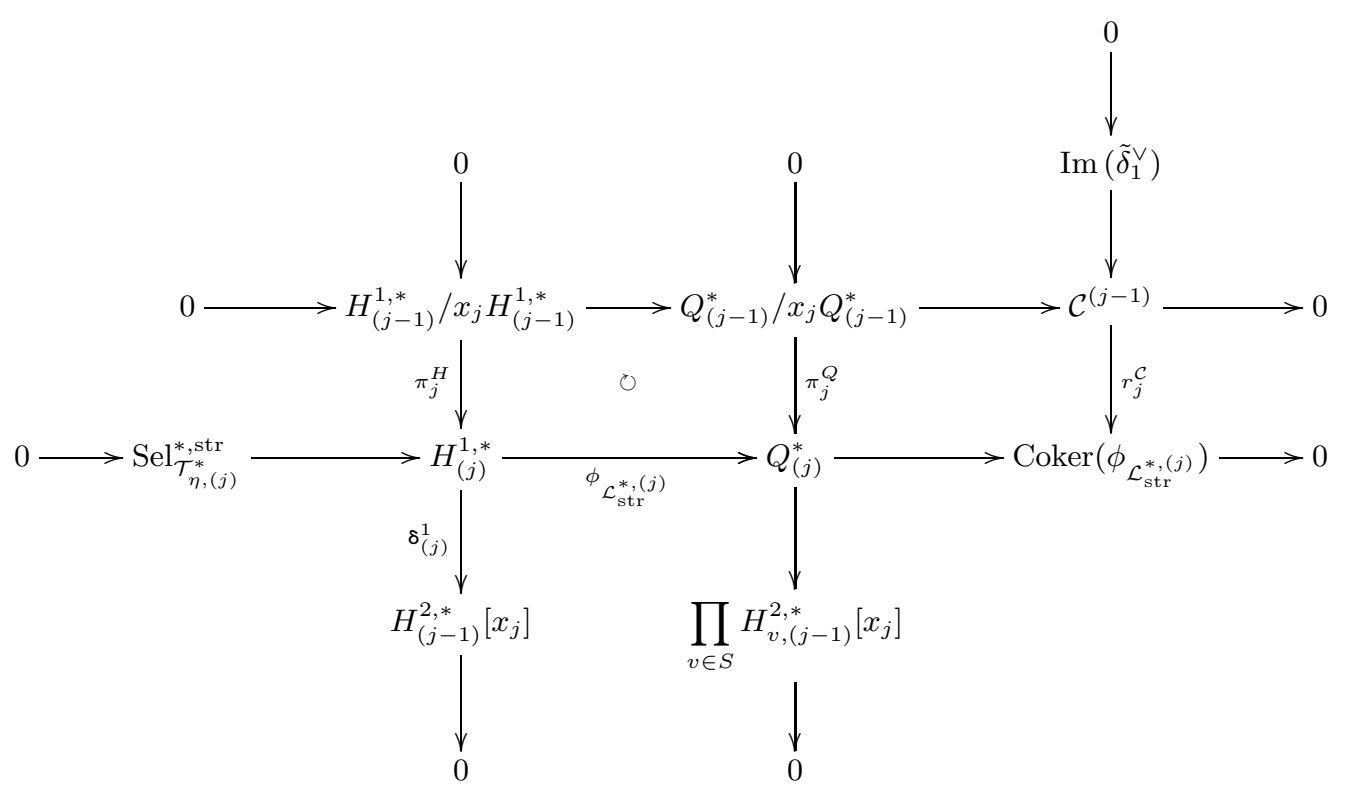


where the left vertical sequence is the one obtained at (3.32) and we use the following abbreviations for each $v$ in $S, i=1,2$ and $k=j-1, j$ :

$$
\operatorname{Sel}_{\mathcal{T}_{\eta,(j)}^{*}, \mathrm{str}}^{*}:=\operatorname{Sel}_{\mathcal{L}_{\mathrm{str}}^{*,(j)}}\left(F, \mathcal{T}_{\eta,(j)}^{*}\right), \quad H_{v,(k)}^{i, *}:=H_{\mathrm{cts}}^{i}\left(F_{v}, \mathcal{T}_{\eta,(k)}^{*}\right)
$$

in addition to the abbreviations (3.26) and (3.33). The top and bottom rows of (3.37) are the sequences (3.32) and (3.30) for $k=j$ respectively, which are hence exact. The right column is the exact sequence (3.29). Here we replace $\left(\amalg_{\mathcal{A}_{\eta,(j-1)}}^{1}\right)^{\vee}\left[x_{j}\right]$ in (3.29) by $\operatorname{Im}\left(\tilde{\delta}_{1}^{\vee}\right)$ for later convenience in Appendix $\mathbb{B}$. The other columns are also exact due to the cohomological long exact sequence and Lemma 3.38. Since both the maps $\pi_{j}^{H}$ and $\pi_{j}^{Q}$ are induced from the canonical surjection $\pi_{j}^{\mathcal{T}}: \mathcal{T}_{\eta,(j-1)}^{*} \rightarrow \mathcal{T}_{\eta,(j)}^{*}$ of the continuous Galois modules, the functoriality of the restriction maps guarantees the commutativity of the left square. We here verify the commutativity of the right square in (3.37).

Lemma 3.39. The right square in the diagram (3.37) commutes.

Proof. Consider the diagram

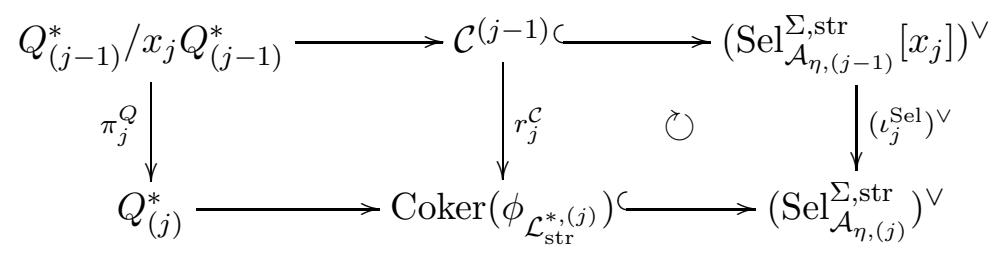

obtained as the composition of the right square in (3.37) and the left commutative square in (3.28). It suffices to prove that the composite square (3.39) commutes since the morphism $\operatorname{Coker}\left(\phi_{\mathcal{L}_{\text {str }}^{*,(j)}}\right) \rightarrow\left(\operatorname{Sel}_{\mathcal{A}_{\eta,(j)}}^{\Sigma \text {,str }}\right)^{\vee}$ in the bottom row is injective. Now recall that we have canonical isomorphisms $Q_{(j-1)}^{*} / x_{j} Q_{(j-1)}^{*} \cong\left(L_{\mathrm{str},(j-1)}\left[x_{j}\right]\right)^{\vee}$ and $Q_{(j)}^{*} \cong\left(L_{\mathrm{str},(j)}\right)^{\vee}$ via the local Tate duality, where we set

$$
L_{\mathrm{str},(k)}=\prod_{v \in S} L_{\mathrm{str}}\left(F_{v}, \mathcal{A}_{\eta,(k)}\right)=\prod_{\substack{v \in S \\ v \notin \Sigma_{p} \cup \Sigma_{p}^{c}}} H_{\mathrm{ur}}^{1}\left(F_{v}, \mathcal{A}_{\eta,(k)}\right) \times \prod_{v \in \Sigma_{p}} H^{1}\left(F_{v}, \mathcal{A}_{\eta,(k)}\right)
$$

for $k=j-1$ and $j$. By construction both of the compositions

$$
\begin{aligned}
& \left(L_{\mathrm{str},(j-1)}\left[x_{j}\right]\right)^{\vee} \cong Q_{(j-1)}^{*} / x_{j} Q_{(j-1)}^{*} \longrightarrow \mathcal{C}^{(j-1)} \longrightarrow\left(\operatorname{Sel}_{\mathcal{A}_{\eta,(j-1)}}^{\Sigma, \mathrm{str}}\left[x_{j}\right]\right)^{\vee}, \\
& \left(L_{\mathrm{str},(j)}\right)^{\vee} \cong Q_{(j)}^{*} \longrightarrow \operatorname{Coker}\left(\phi_{\mathcal{L}_{\mathrm{str}}^{*,(j)}}\right) \longrightarrow\left(\operatorname{Sel}_{\mathcal{A}_{\eta,(j)}^{\Sigma, \mathrm{str}}}\right)^{\vee}
\end{aligned}
$$

of the rows in the diagram (3.39) with the local Tate duality isomorphisms are induced from the dual of the global-to-local restriction map $\phi_{\mathcal{L}_{\mathrm{str}}^{(k)}}: \operatorname{Sel}_{\mathcal{A}_{\eta,(k)}^{\Sigma, \mathrm{str}}} \rightarrow L_{\mathrm{str},(k)}$. Let us define $\iota_{j}^{L}: L_{\mathrm{str},(j-1)} \rightarrow L_{\mathrm{str},(j)}$ as the homomorphism induced from the canonical inclusion $\iota_{j}^{\mathcal{A}}: \mathcal{A}_{\eta,(j)} \hookrightarrow \mathcal{A}_{\eta,(j-1)}$. Then by virtue of the functoriality of the restriction maps, we 
readily obtain a commutative square

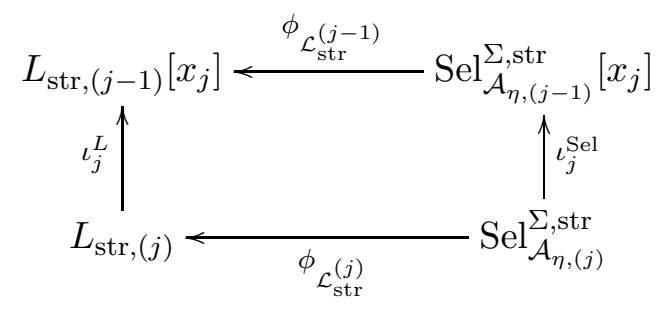

whose Pontrjagin dual coincides with (3.39). Indeed it is straightforward, from the construction of the local Tate pairing as the usual cup product of the group cohomology, to verify that the morphism $\pi_{j}^{Q}$ corresponds to the dual $\left(\iota_{j}^{L}\right)^{\vee}:\left(L_{\mathrm{str},(j-1)}\left[x_{j}\right]\right)^{\vee} \rightarrow\left(L_{\mathrm{str},(j)}\right)^{\vee}$ of $\iota_{j}^{L}$ with respect to the local Tate duality, and thus the proof is completed. Note that $\iota_{j}^{\mathcal{A}}$ and $\pi_{j}^{\mathcal{T}}$ correspond to each other under the Pontrjagin duality.

Now let us consider the coimage $\operatorname{Coim}\left(\phi_{\mathcal{L}_{\text {str }}^{*,(j)}}\right)=H_{(j)}^{1, *} / \operatorname{Sel}_{\mathcal{T}_{\eta,(j)}^{*}}^{* \text { str }}$ of the global-to-local morphism $\phi_{\mathcal{L}_{\text {str }}^{*(j)}}$ and split the bottom row of (3.37) (or in other words, the exact sequence (3.30) for $k=j$ ) into two short exact sequences:

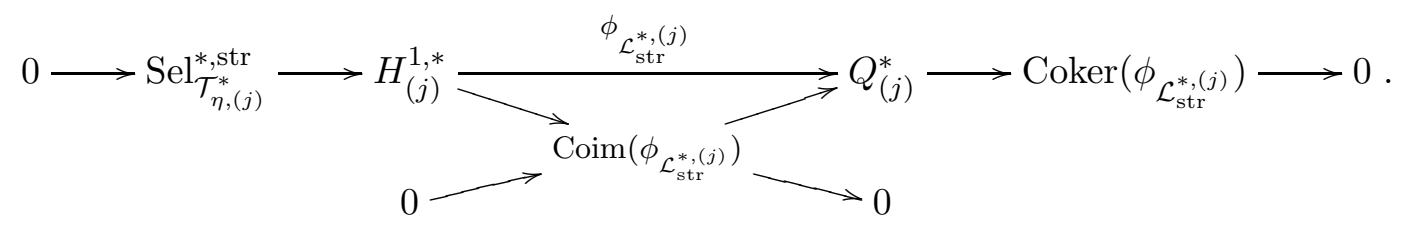

We define $\bar{\pi}_{j}^{H}: H_{(j-1)}^{1, *} / x_{j} H_{(j-1)}^{1, *} \rightarrow \operatorname{Coim}\left(\phi_{\mathcal{L}_{\mathrm{str}}^{*,(j)}}\right)$ as the composition of $\pi_{j}^{H}$ with the natural surjection $H_{(j)}^{1, *} \rightarrow \operatorname{Coim}\left(\phi_{\mathcal{L}_{\text {str }}^{*,(j)}}\right)$, and define $\overline{\mathcal{H}}_{j}^{2, *}$ as the cokernel of $\bar{\pi}_{j}^{H}$. Then diagram (3.37) splits into the two diagrams

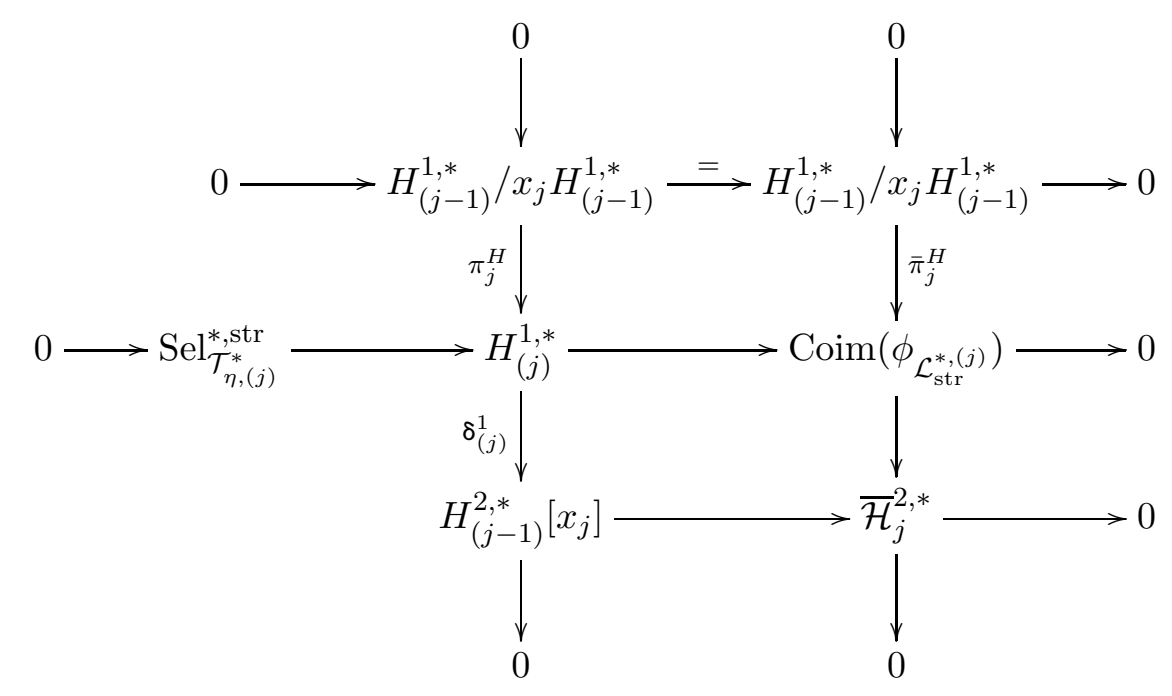


and

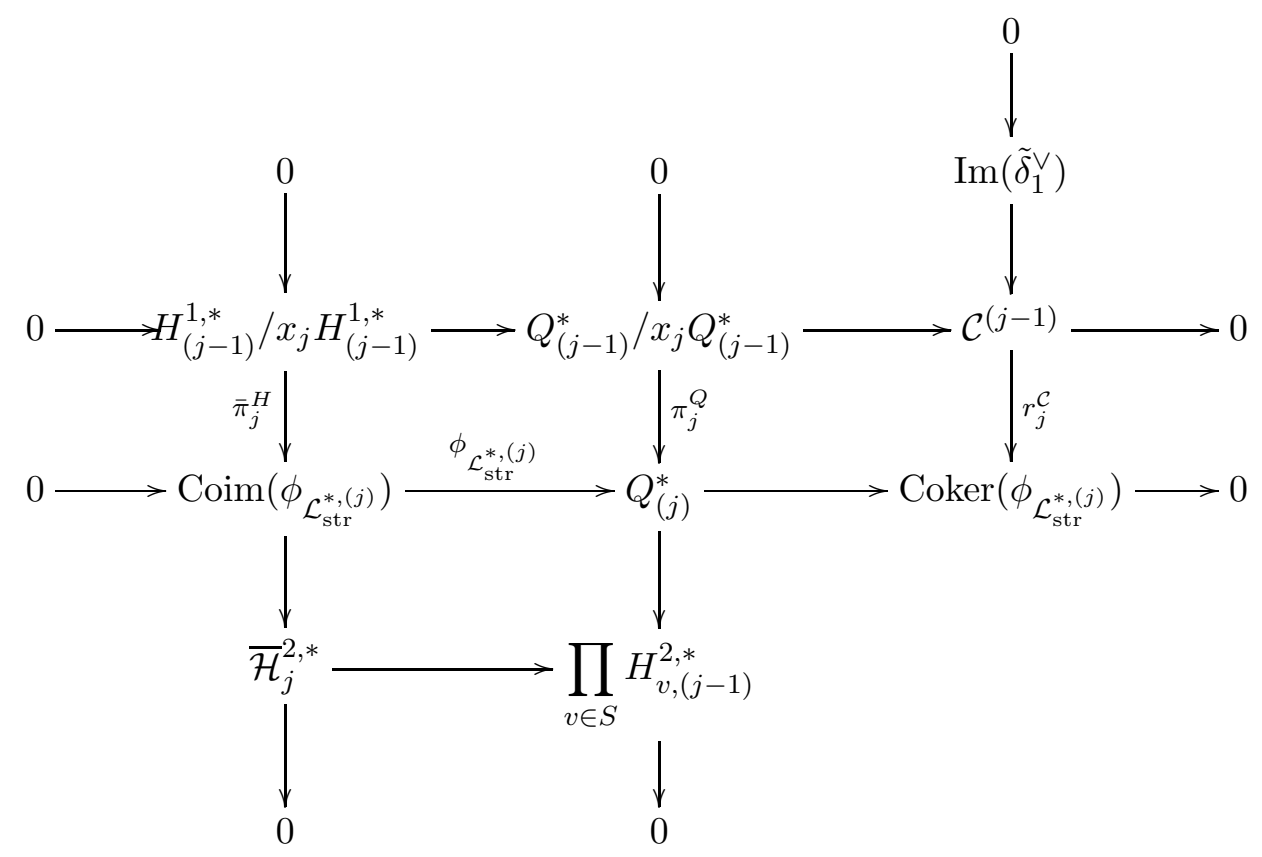

with exact rows and columns. By applying the snake lemma to both the diagrams (3.40) and (3.41), we obtain two exact sequences

$$
0 \longrightarrow \operatorname{Sel}_{\mathcal{L}_{\text {str }}^{*,(j)}}\left(F, \mathcal{T}_{\eta,(j)}^{*}\right) \longrightarrow H_{(j-1)}^{2, *}\left[x_{j}\right] \stackrel{\mathrm{pr}_{H}^{2}}{\longrightarrow} \overline{\mathcal{H}}_{j}^{2, *} \longrightarrow 0
$$

and

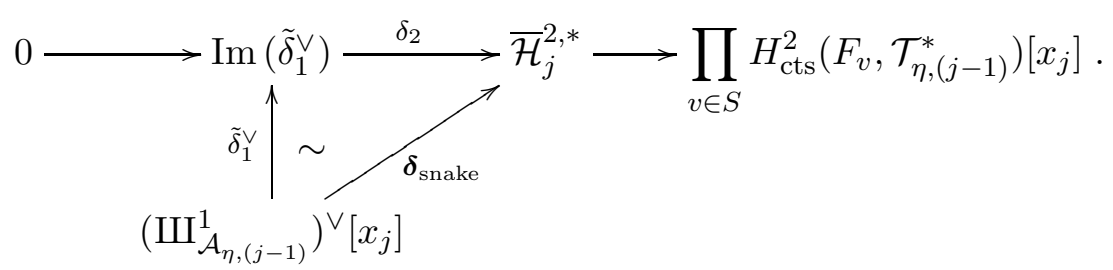

Here we use the symbol " $\boldsymbol{\delta}_{\text {snake" }}$ for the composition $\delta_{2} \circ \tilde{\delta}_{1}^{\vee}$ to indicate that it is constructed as the composition of the connecting homomorphisms derived from the snake lemma. Meanwhile the Poitou-Tate nine-term exact sequence (see [NSW00, (8.6.10)] for example) provides the exact sequence

$$
\prod_{v \in S} H_{\mathrm{cts}}^{0}\left(F_{v}, \mathcal{T}_{\eta,(j-1)}^{*}\right)^{\vee} \longrightarrow\left(H_{(j-1)}^{2, *}\right)^{\vee} \stackrel{\Phi_{\mathrm{PT}}^{\vee}}{\longrightarrow} \amalg_{\mathcal{A}_{\eta,(j-1)}}^{1} \longrightarrow 0
$$

By taking the Pontrjagin duals and the $x_{j}$-torsion parts, we obtain the exact sequence

$$
0 \longrightarrow\left(\amalg_{\mathcal{A}_{\eta,(j-1)}}^{1}\right)^{\vee}\left[x_{j}\right] \stackrel{\Phi_{\mathrm{PT}}}{\longrightarrow} H_{(j-1)}^{2, *}\left[x_{j}\right] \stackrel{\operatorname{Res}_{(j-1)}^{2, *}}{\longrightarrow} \prod_{v \in S} H_{(j-1)}^{2, *}\left[x_{j}\right]
$$

where $\operatorname{Res}_{(j-1)}^{2, *}$ denotes the usual localisation morphism. 
Recall that the homomorphism $\Phi_{\mathrm{PT}}$, which is often called the Poitou-Tate morphism, is the one induced from the Poitou-Tate pairing. See also (B.6) in Appendix B for the definition of $\Phi_{P T}$. The following proposition, whose proof is slightly lengthy and is postponed to Appendix B, is a technical heart of the proof of Proposition 3.37 .

Proposition 3.40. Consider the diagram

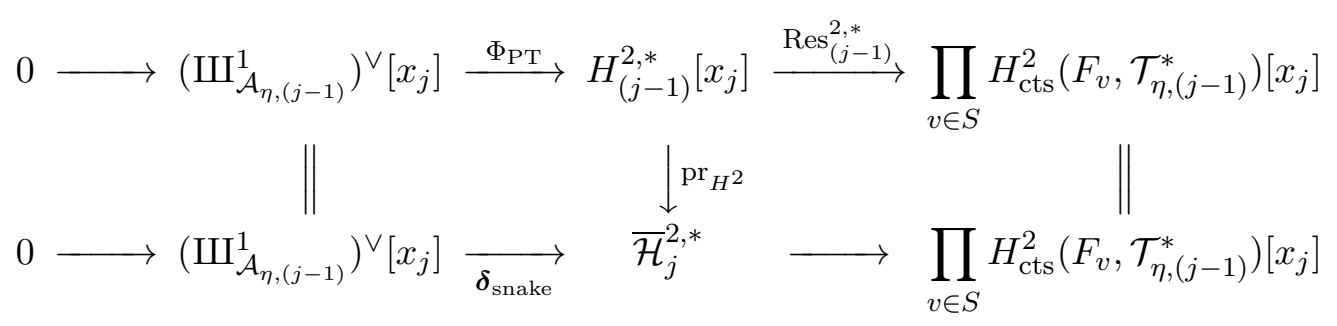

where the top and bottom rows are the exact sequences (3.44) and (3.43) respectively, and the middle vertical morphism $\mathrm{pr}_{H^{2}}$ is the quotient map defined in the short exact sequence (3.42). Then the diagram (3.45) commutes.

The commutativity of the right square in the diagram (3.45) is obvious since both of the horizontal morphisms are induced from the global-to-local morphism

$$
\operatorname{Res}_{(j-1)}^{2, *}: H_{(j-1)}^{2, *} \rightarrow \prod_{v \in S} H_{\mathrm{cts}}^{2}\left(F_{v}, \mathcal{T}_{\eta,(j-1)}^{*}\right) .
$$

Concerning the left square in (3.45), the top horizontal morphism $\Phi_{\mathrm{PT}}$ is the one induced from the Poitou-Tate pairing while the bottom horizontal morphism $\boldsymbol{\delta}_{\text {snake }}$ is the composition of connecting morphisms derived from the snake lemma and the local Tate duality. Therefore we must carefully study the relation between the global Poitou-Tate duality and the local Tate duality to verify the commutativity of the left square. As we shall show below, Proposition 3.37 follows immediately from Proposition 3.40 .

Proof of Proposition 3.37 (admitting Proposition 3.40). We observe from the short exact sequence (3.42) that the kernel of the middle vertical morphism $\mathrm{pr}_{H^{2}}$ in (3.45) is isomorphic to the dual Selmer group $\operatorname{Sel}_{\mathcal{L}_{\text {str }}^{*,(j)}}\left(F, \mathcal{T}_{\eta,(j)}^{*}\right)$. We now readily verify its triviality by an easy diagram chase on (3.45).

3.4.6. Final step: specialisation from two variables to one variable. Suppose that we have already chosen elements $\gamma_{1}, \ldots, \gamma_{d+\delta_{F, p}-1}$ of $\operatorname{Gal}\left(F_{S} / F_{\infty}^{\text {cyc }}\right)$ which satisfy all the conditions $(\Gamma 0)_{d+\delta_{F, p}-1},(\Gamma 1)_{d+\delta_{F, p}-1}$ and $(\Gamma 2)_{d+\delta_{F, p}-1}$ proposed in Section 3.4.5 (the existence of such tuples $\gamma_{1}, \ldots, \gamma_{d+\delta_{F, p}-1}$ is justified in Proposition 3.35). As the final step of the proof of Theorem 3.27, we discuss the cyclotomic specialisation of the strict Selmer group $\mathrm{Sel}_{\mathcal{A}_{\eta}^{\mathrm{CM}}\left[\mathfrak{A}_{\left.d+\delta_{F, p^{-1}}\right]}^{\Sigma, \mathrm{str}}\right]}$. We have already verified in Section 3.4.5 that $\mathrm{Sel}_{\mathcal{A}_{\eta}^{\mathrm{CM}}\left[\mathfrak{A}_{d+\delta_{F, p}-1} \mathrm{s, \textrm {str }}\right.}$ is cotorsion and almost divisible as a $\Lambda_{0}^{\left(d+\delta_{F, p}-1\right)}$-module and the equality (3.22) holds. In this situation the kernel of the natural surjection $\Lambda_{\mathcal{O}}^{\mathrm{CM}} / \mathfrak{A}_{d+\delta_{F, p}-1} \rightarrow \Lambda_{\mathcal{O}}^{\text {cyc }}$ is a principal ideal of $\Lambda_{\mathcal{O}}^{\mathrm{CM}} / \mathfrak{A}_{d+\delta_{F, p}-1}$ generated by $x_{d+\delta_{F, p}}=\gamma_{d+\delta_{F, p}}-1$, where $\gamma_{d+\delta_{F, p}}$ is a lift of a topological generator of $\operatorname{Gal}\left(\widetilde{F} / F_{\infty}^{\mathrm{cyc}}\right) /\left\langle\gamma_{1}, \ldots, \gamma_{d+\delta_{F, p}-1}\right\rangle \cong \mathbb{Z}_{p}$ to $\operatorname{Gal}\left(\widetilde{F} / F_{\infty}^{\mathrm{cyc}}\right)$.

We henceforth denote $\Lambda_{\mathcal{O}}^{\mathrm{CM}} / \mathfrak{A}_{d+\delta_{F, p}-1}$ by $\Lambda_{\mathcal{O}}^{\left(d+\delta_{F, p}-1\right)}$ to simplify the notation. In order to apply the Specialisation Lemma 3.29 to the finitely generated torsion $\Lambda_{\mathcal{O}}^{\left(d+\delta_{F, p}-1\right)}$-module

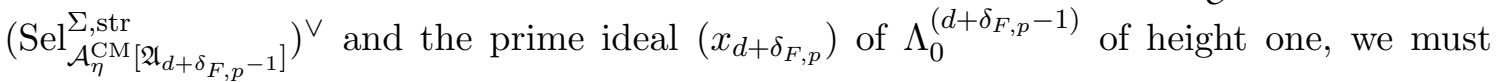


verify that $x_{d+\delta_{F, p}}$ does not divide the characteristic ideal of $\left(\operatorname{Sel}_{\mathcal{A}_{\eta}^{C M}\left[\mathfrak{A}_{d+\delta_{F, p}-1}\right]}^{\Sigma, \operatorname{str}}\right)^{\vee}$. Until the previous steps we could choose a specialising element $x_{j}=\gamma_{j}-1$ avoiding the prime divisors of the characteristic power series of the Pontrjagin dual of the strict Selmer group. At this final step, however, the specialising element $x_{d+\delta_{F, p}}$ (or more precisely, the principal ideal $x_{d+\delta_{F, p}} \Lambda_{0}^{\left(d+\delta_{F, p}-1\right)}$ which it generates) is uniquely determined, and we are not allowed to choose it freely. We still require the following claim:

Claim: Let the notation be as before and let us assume the conditions $\left(\mathrm{IMC}_{F, \psi}\right)$ and $\left(\mathrm{NV}_{\mathcal{L}_{p}^{\text {cyc }}(\vartheta(\eta))}\right)$ (see Theorem 3.27 for details on these conditions). Then the specialising element $x_{d+\delta_{F, p}}$ is relatively prime to the characteristic ideal of the Pontrjagin dual of the $\Lambda_{\mathcal{O}}^{\left(d+\delta_{F, p}-1\right)}$-module $\operatorname{Sel}_{\mathcal{A}_{\eta}^{\mathrm{CM}}\left[\mathfrak{A}_{d+\delta_{F, p}-1}, \mathrm{str}\right.}$.

Proof of the Claim. In order to prove the claim, we assume that $x_{d+\delta_{F, p}}$ does divide the characteristic ideal of $\left(\mathrm{Sel}_{\mathcal{A}_{\eta}^{C M}\left[\mathfrak{A}_{d+\delta_{F, p}-1}\right]}^{\Sigma, \mathrm{str}}\right)^{\vee}$ and deduce contradiction. The cyclotomic specialisation of the characteristic ideal

$$
\begin{aligned}
& \left(\operatorname{Char}_{\Lambda_{\mathcal{O}}^{\mathrm{CM}}}\left(\operatorname{Sel}_{\mathcal{A}_{\eta}^{\mathrm{CM}}}^{\Sigma, \mathrm{str}}\right)^{\vee}\right) \otimes_{\Lambda_{\mathcal{O}}^{\mathrm{CM}}} \Lambda_{\mathcal{O}}^{\mathrm{cyc}} \\
& \quad=\left(\operatorname{Char}_{\Lambda_{\mathcal{O}}^{\left(d+\delta_{F, p}-1\right)}}\left(\operatorname{Sel}_{\mathcal{A}_{\eta}^{\mathrm{CM}}\left[\mathfrak{A}_{d+\delta_{F, p}-1}\right]}^{\Sigma, \mathrm{str}}\right)^{\vee}\right) \otimes_{\Lambda_{\mathcal{O}}^{\left(d+\delta_{F, p}-1\right)}} \Lambda_{\mathcal{O}}^{\left(d+\delta_{F, p}-1\right)} / x_{d+\delta_{F, p}} \Lambda_{\mathcal{O}}^{\left(d+\delta_{F, p}-1\right)}
\end{aligned}
$$

is then trivial. On the other hand, we obtain the equality of ideals of $\widehat{\mathcal{O}}^{\mathrm{ur}}[[\operatorname{Gal}(\widetilde{F} / F)]]$

$$
\operatorname{Char}_{\Lambda_{\mathcal{O}}^{\mathrm{CM}}}\left(\operatorname{Sel}_{\mathcal{A}_{\eta}^{\mathrm{CM}}}^{\Sigma, \mathrm{str}}\right)^{\vee}=\operatorname{Tw}_{\eta^{\mathrm{gal}} \psi^{-1}}\left(\operatorname{Char}_{\Lambda_{\mathcal{O}}^{\mathrm{CM}}}\left(\operatorname{Sel}_{\mathcal{A}_{\psi}^{\mathrm{CM}}}^{\Sigma, \mathrm{str}}\right)^{\vee}\right)=\left(\operatorname{Tw}_{\eta^{\mathrm{gal}}} \psi^{-1}\left(\mathcal{L}_{p}^{\Sigma}(\psi)\right)\right)
$$

by the Iwasawa main conjecture $\left(\operatorname{IMC}_{F, \psi}\right)$ for $F$ and $\psi$. We thus see from the equations (3.46) and (3.47) that the image $\mathcal{L}_{p, \mathrm{CM}}^{\mathrm{cyc}}(\eta)$ of $\mathrm{Tw}_{\eta^{\mathrm{gal}} \psi^{-1}}\left(\mathcal{L}_{p}^{\Sigma}(\psi)\right)$ in the Iwasawa algebra $\widehat{\mathcal{O}}^{\text {ur }}\left[\left[\operatorname{Gal}\left(F^{+}\left(\mu_{p^{\infty}}\right) / F^{+}\right)\right]\right]$is trivial. On the other hand, we have already observed in Corollary 2.25 that the element $\mathcal{L}_{p, \mathrm{CM}}^{\mathrm{cyc}}(\eta)$ is a nonzero multiple of the cyclotomic $p$-adic $L$-function $\mathcal{L}_{p}^{\text {cyc }}(\vartheta(\eta))$ of $\vartheta(\eta)$ in each component of the semilocal Iwasawa algebra $\widehat{\mathcal{O}}^{\text {ur }}\left[\left[\operatorname{Gal}\left(F^{+}\left(\mu_{p^{\infty}}\right) / F^{+}\right)\right]\right] \otimes_{\mathbb{Z}_{p}} \mathbb{Q}_{p}$. The nontriviality assumption $\left(\operatorname{NV}_{\mathcal{L}_{p}^{\text {cyc }}(\vartheta(\eta))}\right)$ thus leads us to contradiction, which completes the proof of the claim.

Due to the claim, we can apply the Specialisation Lemma 3.29 to $\left(\operatorname{Sel}_{\mathcal{A}_{\eta}^{\mathrm{CM}}\left[\mathfrak{A}_{d+\delta_{F, p}-1}\right]}^{\mathrm{s, \textrm {str }}}\right)^{\vee}$ and $x_{d+\delta_{F, p}} \Lambda_{0}^{\left(d+\delta_{F, p}-1\right)}$. We thus observe that the strict Selmer group $\operatorname{Sel}_{\mathcal{A}_{\eta}^{\text {cyc }}}^{\Sigma \text {,str }}$ of the cyclotomic deformation $\mathcal{A}_{\eta}^{\text {cyc }}$ of $\eta$ is cotorsion as a $\Lambda_{\mathcal{O}}^{\text {cyc }}$-module, and and obtain the basechange compatibility of the characteristic ideal with respect to the cyclotomic specialisation:

$$
\left(\operatorname{Char}_{\Lambda_{\mathcal{O}}^{\mathrm{CM}}}\left(\operatorname{Sel}_{\mathcal{A}_{\eta}^{\mathrm{CM}}}^{\Sigma, \mathrm{str}}\right)^{\vee}\right) \otimes_{\Lambda_{\mathcal{O}}^{\mathrm{CM}}} \Lambda_{\mathcal{O}}^{\text {cyc }}=\operatorname{Char}_{\Lambda_{\mathcal{O}}^{\text {cyc }}}\left(\operatorname{Sel}_{\mathcal{A}_{\eta}^{\text {cyc }}}^{\Sigma, \mathrm{str}}\right)^{\vee} .
$$

This is the end of the proof of Theorem 3.27

3.5. Application to the Iwasawa Main Conjecture. We shall prove the main theorem of this article (Theorem 3.41). Firstly let us recall the notation and the settings. As in Section 1, let $p$ be an odd prime number and let $F^{+}$be a totally real number field of degree $d$ satisfying the unramifiedness condition $\left(\mathrm{unr}_{F^{+}}\right)$. Consider a nearly $p$-ordinary $p$-stabilised newform $f=\vartheta(\eta)$ with complex multiplication defined on $F^{+}$, which is associated to a größencharacter $\eta$ of type $\left(A_{0}\right)$ on a totally imaginary quadratic extension $F$ of $F^{+}$ satisfying the $p$-ordinarity condition $\left(\operatorname{ord}_{F / F^{+}}\right.$). We may assume that $\eta$ is ordinary with 
respect to an appropriate $p$-ordinary CM type $\Sigma$ of $F$, which we henceforth fix. Finally we choose and fix a branch character $\psi$ associated to $\eta$ (see Definition 3.8).

Theorem 3.41 (Theorem (D). Let the notation be as above and assume that all the following conditions are fulfilled:

- the nontriviality condition $(\mathrm{ntr})_{\mathfrak{P}}$ for every place $\mathfrak{P}$ of $F$ contained in $\Sigma_{p}^{c}$;

- the $\left(d+\delta_{F, p}+1\right)$-variable Iwasawa main conjecture $\left(\mathrm{IMC}_{F, \psi}\right)$ for the CM field $F$ and the branch character $\psi$;

- the nontriviality condition $\left(\mathrm{NV}_{\mathcal{L}_{p}^{\mathrm{CM}}(\vartheta(\eta))}\right)$ for the cyclotomic p-adic L-function associated to $\vartheta(\eta)$.

Then the cyclotomic Iwasawa main conjecture for the Hilbert cuspform $\vartheta(\eta)$ is true up to $\mu$-invariants; that is, the equality

$$
\left(\mathcal{L}_{p}^{\text {cyc }}(\vartheta(\eta))\right)=\operatorname{Char}_{\Lambda_{\mathcal{O}}^{\text {cyc }}}\left(\operatorname{Sel}_{\mathcal{A}_{\vartheta(\eta)}^{\text {cyc }}}^{\vee}\right)
$$

holds as an equation of ideals of $\widehat{\mathcal{O}}^{\mathrm{ur}}\left[\left[\operatorname{Gal}\left(F^{+}\left(\mu_{p^{\infty}}\right) / F^{+}\right)\right]\right] \otimes_{\mathbb{Z}_{p}} \mathbb{Q}_{p}$. Furthermore the equality (3.48) holds as an equation of ideals of $\Lambda_{\mathcal{O}}^{\mathrm{cyc}}$ if Conjecture 2.26] is true.

Proof. The claim follows directly from Corollary 2.25 and Theorem 3.27

Remark 3.42 (On the condition $\left(\mathrm{IMC}_{F, \psi}\right)$ ). Concerning the multi-variable Iwasawa main conjecture for CM number fields $\left(\mathrm{IMC}_{F, \psi}\right)$, Ming-Lun Hsieh [Hsi14] has recently obtained several results over the $(d+1)$-variable Iwasawa algebra associated to the Galois group of the compositum of the anticyclotomic $\mathbb{Z}_{p}^{d}$-extension ( $d$-variable) and the cyclotomic $\mathbb{Z}_{p^{-}}$ extension (1-variable). If we assume the Leopoldt conjecture, which claim that the Leopoldt defect $\delta_{F, p}$ would equal zero, the Iwasawa algebra above coincides with $\mathcal{O}[[\operatorname{Gal}(\widetilde{F} / F)]]$ (which is isomorphic to each component of $\Lambda_{\mathcal{O}}^{\mathrm{CM}}$ ). Thus Hsieh's result [Hsi14, Theorem 8.16] combined with Leopoldt conjecture implies a one-sided divisibility relation

$$
\mathcal{L}_{p}^{\mathrm{CM}}(\psi) \mid \operatorname{Char}_{\Lambda_{\mathcal{O}}^{\mathrm{CM}}}\left(\operatorname{Sel}_{\mathcal{A}_{\eta}^{\mathrm{CM}}}^{\vee}\right)
$$

in our cases under certain technical assumptions. Also [Hsi14, Theorem 8.17, Theorem 8.18] combined with Leopoldt conjecture implies the whole equality

$$
\mathcal{L}_{p}^{\mathrm{CM}}(\psi)=\operatorname{Char}_{\Lambda_{\mathcal{O}}^{\mathrm{CM}}}\left(\operatorname{Sel}_{\mathcal{A}_{\eta}^{\mathrm{CM}}}^{\vee}\right)
$$

holds in our cases under certain technical assumptions.

Remark 3.43 (On the nontriviality of the cyclotomic $p$-adic $L$-functions). We here discuss the validity of the nontriviality condition $\left(\mathrm{NV}_{f}\right)$ for general Hilbert cuspforms. As in Theorem 2.15, let $f$ be a normalised nearly $p$-ordinary eigencuspform in $S_{\kappa}(\mathfrak{N}, \underline{\varepsilon} ; \overline{\mathbb{Q}})$ which is stabilised at $p$. If the region of the convergence of the (twisted) Dirichlet series

$$
L\left(f, \phi^{-1}, s\right)=\sum_{\mathfrak{a} \subset \mathfrak{r}_{F^{+}}} \frac{C(\mathfrak{a} ; f) \phi^{-1}(\mathfrak{a})}{\mathcal{N} \mathfrak{a}^{s}}
$$

contains at least one of $\kappa_{1}^{\max }+1, \kappa_{1}^{\max }+2, \ldots, \kappa_{2, \min }$, the cyclotomic $p$-adic $L$-function $\mathcal{L}_{p}^{\text {cyc }}(f)$ associated to $f$ is obviously nontrivial. Indeed the value $L(f, \phi, j)$ of the (complex) $L$-function at such a point never equals zero, and hence the nontriviality of $\mathcal{L}_{p}^{\text {cyc }}(f)$ immediately follows from the interpolation formula (2.14). The Ramanujan-Petersson conjecture for Hilbert modular forms, which was verified by Brylinski, Labesse [BrLa84, 
Théorème 3.4.5] and Blasius [Bl06, Theorem 1], suggests that the Dirichlet series (3.49) absolutely converges in the region $\operatorname{Re}(s)>\frac{[\kappa]}{2}+1$, and thus the nontriviality of the cyclotomic $p$-adic $L$-function of $\mathcal{L}_{p}^{\text {cyc }}(f)$ is automatically deduced when the inequality

$$
\kappa_{2, \min }>\frac{[\kappa]}{2}+1
$$

holds. In contrast, it is very hard to verify that the critical values $L(f, \phi, j)$ of the (complex) $L$-function do not vanish when none of the critical points are contained in the region of convergence. For elliptic modular forms, Rohrlich has verified the nonvanishing of such critical values in general situations [R084, Ro88], and thus the condition $\left(\mathrm{NV}_{f}\right)$ is fulfilled for elliptic modular forms. For Hilbert modular forms, however, there have not been enough results yet to verify the condition $\left(\mathrm{NV}_{f}\right)$ for general $f$.

\section{Appendix A. Complex multiplication of Hilbert modular Cuspforms}

Let $F^{+}$be a totally real number field and consider a Hilbert eigencuspform $f$ defined over $G L(2)_{F^{+}}$of cohomological weight $\kappa$, level $\mathfrak{N}$ and nebentypus $\underline{\varepsilon}$, and suppose that $[\kappa]$ is strictly greater than zero. We further assume that $f$ is a primitive form in the sense of Miyake [Mi71], and denote by $\mathbb{Q}_{f}$ the Hecke field associated to $f$. Then due to results of many people including Ohta Oh83, Carayol [Ca86, Wiles Wi88, Taylor [Tay89 and Blasius and Rogawski BlRo94, we can canonically attach to $f$ a strictly compatible system $\left(\rho_{f, \lambda}\right)_{\lambda}$ of 2-dimensional $\lambda$-adic representations of the absolute Galois group $G_{F^{+}}$of $F^{+}$; namely, for each finite place $\lambda$ of $F^{+}$with residue characteristic $\ell$, the 2 -dimensional $\lambda$-adic representation

$$
\rho_{f, \lambda}: G_{F^{+}} \rightarrow \operatorname{Aut}_{\mathbb{Q}_{f, \lambda}} V_{f, \lambda}
$$

is unramified outside $\ell \mathfrak{N}$ and characterised by the formulae

$$
\operatorname{Tr} \rho_{f, \lambda}\left(\operatorname{Frob}_{\mathfrak{q}}\right)=C(\mathfrak{q} ; f), \quad \operatorname{det} \rho_{f, \lambda}\left(\text { Frob }_{\mathfrak{q}}\right)=\chi_{\ell, \text { cyc }^{-1}} \varepsilon_{+}^{\text {gal }}\left(\text { Frob }_{\mathfrak{q}}\right)
$$

for each prime ideal $\mathfrak{q}$ of $F^{+}$relatively prime to $\ell \mathfrak{N}$ where $\chi_{\ell \text {,cyc }}$ denotes the $\ell$-adic cyclotomic character. Further, each Galois representation $\rho_{f, \lambda}$ is known to be irreducible (see Tay95, Theorem 3.1]).

The following statement is widely known for elliptic modular forms due to Ribet [Ri77].

Proposition A.1. Let $f$ be a primitive Hilbert cuspform as above. Then the following three statements on $f$ are equivalent;

(1) the primitive form $f$ has complex multiplication;

(2) the absolute Galois group $G_{F^{+}}$contains an open subgroup $H$ of index two such that the image of $H$ under the associated $\lambda$-adic Galois representation $\rho_{f, \lambda}$ is abelian for every finite place $\lambda$ of $\mathbb{Q}_{f}$;

(3) there exist a totally imaginary quadratic extension $F$ of $F^{+}$and a primitive größencharacter $\eta$ (in the sense that the modulus of $\eta$ coincides with its conductor) of type $\left(A_{0}\right)$ on $F$ such that $f$ coincides with the theta lift $\vartheta(\eta)$ of $\eta$. Furthermore there exists a CM type $\Sigma$ of $F$ such that the größencharacter $\eta$ is admissible with respect to $\Sigma$ and its infinity type is described as $\sum_{\sigma \in \Sigma} \kappa_{1,\left.\sigma\right|_{F^{+}}} \sigma+\sum_{\bar{\sigma} \in \Sigma^{c}} \kappa_{2,\left.\bar{\sigma}\right|_{F^{+}}} \bar{\sigma}$.

In the cases above, $\rho_{f, \lambda}$ is isomorphic to the induced representation $\operatorname{Ind}_{F}^{F^{+}} \eta^{\text {gal }}$ of the 1-dimensional $\lambda$-adic representation $\eta^{\text {gal }}: G_{F} \rightarrow \mathbb{Q}_{f, \lambda}^{\times}$which corresponds to the $\ell$-adic avatar $\hat{\eta}_{\ell}$ of the größencharacter $\eta$ of type $\left(A_{0}\right)$ introduced in $(3)$. Here we consider the $\ell$-adic avatar $\hat{\eta}_{\ell}$ with respect to a specific embedding $\overline{\mathbb{Q}} \hookrightarrow \overline{\mathbb{Q}}_{\ell}$ which induces $\lambda$ on $\mathbb{Q}_{f}$. 
The proof of the proposition proceeds analogously to Ribet's arguments in [Ri77, Sections 3 and 4]. It is based upon a precise study of the Galois representation $\rho_{f, \lambda}$ associated to the Hilbert modular cuspform $f$ with complex multiplication.

Remark A.2. Due to the lack of appropriate references, we decided to give a proof of Proposition A.1 in this appendix. After the first redaction of the article, we learned that some of the results in Proposition A.1, say the equivalence between the statement (1) and the statement (3), has been already proved in [LL79, Proposition 6.5] with the language of automorphic representation. We still leave the proof of Proposition A.1 below believing that the proof with the language of Galois representation has its own value.

Proof of Proposition A.1. We first prove that the statement (3) implies the statement (1). Assume that $f$ is obtained as the theta lift $\vartheta(\eta)$ of a primitive größencharacter $\eta$ of type $\left(A_{0}\right)$ defined on a totally imaginary quadratic extension $F$ of $F^{+}$. Then one easily observes by the construction of the theta lift (refer to Proposition 2.13 for details) that the Fourier coefficient $C(\mathfrak{q}, \vartheta(\eta))$ at a prime ideal $\mathfrak{q}$ of $F^{+}$equals zero if and only if $\mathfrak{q}$ is inert or ramified in $F$, or equivalently, the evaluation of the quadratic character $\nu_{F / F^{+}}: \mathbb{A}_{F^{+}}^{\times} \rightarrow \mathbb{C}^{\times}$ associated to the quadratic extension $F / F^{+}$at $\mathfrak{q}$ equals either 0 or -1 . This is equivalent to the validity of the equation (2.7) when one replaces the character $\nu$ appearing in (2.7) by the quadratic character $\nu_{F / F^{+}}$.

Next we prove that the statement (1) implies the statement (2). Assume that $f$ has complex multiplication by a nontrivial größencharacter $\nu$ on $\mathbb{A}_{F^{+}}^{\times}$: then $\nu$ is a quadratic character (see the arguments in Section 2.1.5). The equation (2.7) for each $\mathfrak{q}$ in a set of prime ideals of $F^{+}$of density one implies that the traces $\operatorname{Tr} \rho_{f, \lambda}\left(\right.$ Frob $\left._{\mathfrak{q}}\right)$ and $\operatorname{Tr} \rho_{f \otimes \nu, \lambda}\left(\operatorname{Frob}_{\mathfrak{q}}\right)$ coincide for each $\mathfrak{q}$ in the same set of prime ideals. Since both $\rho_{f, \lambda}$ and $\rho_{f \otimes \nu, \lambda}$ are irreducible by Tay95, Theorem 3.1], Čebotarev's density theorem suggests that they are isomorphic to each other as $\lambda$-adic representations of $G_{F^{+}}$. In other words, there exists a $(2 \times 2)$-matrix $M$ in $G L_{2}\left(\mathbb{Q}_{f, \lambda}\right)$ such that the equality

$$
\rho_{f, \lambda}(g)=M \rho_{f \otimes \nu, \lambda}(g) M^{-1}=\nu(g) M \rho_{f, \lambda}(g) M^{-1}
$$

holds for an arbitrary element $g$ of $G_{F^{+}}$when we fix noncanonical identifications $V_{f, \lambda} \cong \mathbb{Q}_{f, \lambda}^{\oplus 2}$ and $V_{f \otimes \nu, \lambda} \cong \mathbb{Q}_{f, \lambda}^{\oplus 2}$. Let us denote by $H$ the kernel of the quadratic character $\nu$, which is a subgroup of index two of $G_{F^{+}}$. Take an element $g_{0}$ from the complement of $H$ in $G_{F^{+}}$ and set $T=\rho_{f, \lambda}\left(g_{0}\right)$. We then obtain the equality $T=-M T M^{-1}$ by (A.1), from which we readily observe that $M$ is semisimple but not a scalar matrix. The equation (A.1) also implies that the image of $H$ under $\rho_{f, \lambda}$ is contained in the commutant of the semisimple, nonscalar matrix $M$, and thus it is abelian. The subgroup $H$ satisfies all the required condition in the statement (2), and hence the statement (2) follows from (1). We remark that since $H$ is defined as the kernel of the rational quadratic character $\nu$, it is determined independently of the choice of a finite place $\lambda$ of $\mathbb{Q}_{f}$.

We finally verify that the statement (2) implies the statement (3), which is a crucial part of the proof. Suppose that $G_{F^{+}}$contains an open subgroup $H$ of index two such that the image of $H$ under each $\lambda$-adic representation $\rho_{f, \lambda}$ is abelian. Let $F$ denote the subfield of $\overline{\mathbb{Q}}$ corresponding to $H$, which is a quadratic extension of $F^{+}$. By assumption, the restriction $\left.\rho_{f, \lambda}\right|_{H}$ of $\rho_{f, \lambda}$ to $H$ is a semisimple, abelian $\lambda$-adic representation of $H$ for every finite place $\lambda$ of $\mathbb{Q}_{f}$, and therefore $\left.\rho_{f, \lambda}\right|_{H}$ is locally algebraic due to the local algebraicity theorem proved by Serre [Se98, Chapter III. Section 3.] and Henniart [He82, Section 6.]. In other words, there exists a morphism of algebraic groups $r: \mathbb{S}_{\mathfrak{m} / \mathbb{Q}_{f}} \rightarrow G L(2) / \mathbb{Q}_{f}$ giving rise to the strictly compatible system $\left(\left.\rho_{f, \lambda}\right|_{H}\right)_{\lambda}$ of the $\lambda$-adic representations $\left.\rho_{f, \lambda}\right|_{H}$ of $H$. Here 
$\mathbb{S}_{\mathfrak{m} / \mathbb{Q}}$ denotes Serre's algebraic group associated to the field $F$ and an appropriate integral modulus $\mathfrak{m}$ of $F$ (called the modulus of definition) and $\mathbb{S}_{\mathfrak{m} / \mathbb{Q}_{f}}$ denotes its basechange over $\mathbb{Q}_{f}$. Serre's algebraic group $\mathbb{S}_{\mathfrak{m} / \mathbb{Q}}$ is by construction an extension of the ray class group $\mathrm{Cl}(F)_{\mathfrak{m}}$ of $F$ modulo $\mathfrak{m}$ (regarded as a constant group scheme) by a certain algebraic torus $T_{\mathfrak{m}}$ defined over $\mathbb{Q}$, and it is in particular of multiplicative type. See [Se98, Chapter II] for details on properties of $\mathbb{S}_{\mathfrak{m} / \mathbb{Q}}$. The algebraic representation $r$ is thus $\left(\mathbb{Q}_{f}\right.$-rational and $)$ semisimple; namely there exists a $\mathbb{Q}_{f}$-rational pair of algebraic characters $\left(\eta_{1}^{\text {alg }}, \eta_{2}^{\text {alg }}\right)$ of $\mathbb{S}_{\mathfrak{m}} / \overline{\mathbb{Q}}$ (in the sense that the summation $\eta_{1}^{\text {alg }}+\eta_{2}^{\text {alg }}$ is invariant under the natural action of $\operatorname{Gal}\left(\overline{\mathbb{Q}} / \mathbb{Q}_{f}\right)$ ) such that $r$ is isomorphic to the direct sum $\eta_{1}^{\text {alg }} \oplus \eta_{2}^{\text {alg }}$ over $\overline{\mathbb{Q}}$. For each $i=1,2$ and each finite place $\lambda$ of $\mathbb{Q}_{f}$, let $\eta_{i, \lambda}^{\text {gal }}$ denote the 1-dimensional $\lambda$-adic representation associated to $\eta_{i}^{\text {alg }}$, and let $\eta_{i}$ denote the größencharacter of type $\left(A_{0}\right)$ on $F$ associated to $\eta_{i}^{\text {alg }}$. Then by construction $\eta_{i, \lambda}^{\text {gal }}$ is the Galois character of $H$ associated to the $\ell$-adic avatar $\hat{\eta}_{i, \ell}$ of $\eta_{i}$, and $\left.\rho_{f, \lambda}\right|_{H}$ is equivalent to the direct sum of $\eta_{1, \lambda}^{\text {gal }}$ and $\eta_{2, \lambda}^{\text {gal }}$. Now let $c$ denote the generator of the quotient group $G_{F^{+}} / H$, and let us take its arbitrary lift $\tilde{c}$ to $G_{F^{+}}$. We define the $c$-conjugation $\left.\rho_{f, \lambda}\right|_{H} ^{c}$ of $\left.\rho_{f, \lambda}\right|_{H}$ by $\left.\rho_{f, \lambda}\right|_{H} ^{c}(h)=\left.\rho_{f, \lambda}\right|_{H}\left(\tilde{c} h \tilde{c}^{-1}\right)$ for each element $h$ in $H$. Note that $\left.\rho_{f, \lambda}\right|_{H} ^{c}$ is well defined independently of the choice of $\tilde{c}$. Then, since $\rho_{f, \lambda}$ is defined on $G_{F^{+}}$, the trace of $\left.\rho_{f, \lambda}\right|_{H} ^{c}$ obviously coincides with that of $\left.\rho_{f, \lambda}\right|_{H}$. Furthermore both $\left.\rho_{f, \lambda}\right|_{H}$ and $\left.\rho_{f, \lambda}\right|_{H} ^{c}$ are semisimple, and hence they are isomorphic to each other by Cebotarev's density theorem. Consequently either of the followings two cases occurs: $\left(\eta_{i, \lambda}^{c}=\eta_{i, \lambda}\right.$ for $\left.i=1,2\right)$ or $\left(\eta_{1, \lambda}^{c}=\eta_{2, \lambda}\right.$ and $\left.\eta_{2, \lambda}^{c}=\eta_{1, \lambda}\right)$. If the former case occurs, the equality $\rho_{f, \lambda}(\tilde{c} h)=\rho_{f, \lambda}(h \tilde{c})$ holds for every $h$ in $H$. This means that $\rho_{f, \lambda}$ is an abelian representation of $G_{F^{+}}$, which contradicts the irreducibility of $\rho_{f, \lambda}$. Therefore the latter case does occur, and in particular the conjugation by $\rho_{f, \lambda}(\tilde{g})$ corresponds to the interchange of $\eta_{1, \lambda}^{\text {gal }}$ and $\eta_{2, \lambda}^{\text {gal }}$ if $\tilde{g}$ is an element of the complement of $H$ in $G_{F^{+}}$. From this fact we readily verify that the image $\rho_{f, \lambda}(\tilde{g})$ of such $\tilde{g}$ is conjugate to a matrix both of whose diagonal entries equal zero, and thus $\operatorname{Tr} \rho_{f, \lambda}\left(\operatorname{Frob}_{\mathfrak{q}}\right)$ is trivial for a prime ideal $\mathfrak{q}$ of $F^{+}$which inerts in $F$. If a prime ideal $\mathfrak{q}$ of $F^{+}$splits completely in $F$, on the contrary, the decomposition group at $\mathfrak{q}$ is naturally identified with $H$. Combining these observations, we obtain the following formula for each prime ideal $\mathfrak{q}$ which does not divide $\ell \mathfrak{N}$ (note that $\eta_{2, \lambda}^{\text {gal }}\left(\operatorname{Frob}_{\mathfrak{Q}}\right)=\eta_{1, \lambda}^{\text {gal }}\left(\tilde{c}\right.$ Frob $\left._{\mathfrak{Q}} \tilde{c}^{-1}\right)=\eta_{1, \lambda}^{\text {gal }}\left(\right.$ Frob $\left._{\mathfrak{Q}^{c}}\right)$ holds $)$ :

$$
\operatorname{Tr} \rho_{f, \lambda}\left(\operatorname{Frob}_{\mathfrak{q}}\right)= \begin{cases}\eta_{1, \lambda}^{\text {gal }}\left(\operatorname{Frob}_{\mathfrak{Q}}\right)+\eta_{1, \lambda}^{\text {gal }}\left(\text { Frob }_{\mathfrak{Q}^{c}}\right) & \text { if } \mathfrak{q} \text { splits in } F \text { as } \mathfrak{q}=\mathfrak{Q}^{c} \\ 0 & \text { otherwise }\end{cases}
$$

This calculation (combined with Čebotarev's density theorem and the irreducibility of $\rho_{f, \lambda}$ ) implies that $\rho_{f, \lambda}$ is isomorphic to the induced representation $\operatorname{Ind}_{H}^{G_{F}+} \eta_{1, \lambda}^{\text {gal }}$ of $\eta_{1, \lambda}^{\text {gal }}$. We remark that this is canonically extended to an isomorphism between the strict compatible systems $\left(\rho_{f, \lambda}\right)_{\lambda}$ and $\left(\operatorname{Ind}_{H}^{G_{F}^{+}} \eta_{1, \lambda}^{\text {gal }}\right)_{\lambda}$; namely, $\rho_{f, \lambda}$ is isomorphic to $\operatorname{Ind}_{H}^{G_{F}+} \eta_{1, \lambda}^{\text {gal }}$ for every finite place $\lambda$ of $\mathbb{Q}_{f}$ which is not contained in either of the exceptional sets of the two strictly compatible systems.

Now, we verify that the field $F$ corresponding to the subgroup $H$ of $G_{F^{+}}$is a purely imaginary quadratic extension of $F^{+}$(and thus $F$ is in particular a CM number field). Indeed if $F$ is not purely imaginary over $F^{+}$, every algebraic character of $\mathbb{T}_{\mathfrak{m}}$ is described as an integral power of the norm character (see [Se98, Chapter II, Section 3.3]). In particular, 
each $\lambda$-adic character $\eta_{i, \lambda}^{\text {gal }}$ is described as

$$
\eta_{i, \lambda}^{\mathrm{gal}}=\chi_{\ell, \mathrm{cyc}}^{-n_{i}} \eta_{i, \lambda}^{f}
$$

for a certain integer $n_{i}$ and a certain character $\eta_{i, \lambda}^{f}$ of $H$ of finite order. Then the determinants of $\rho_{f, \lambda}$ and $\operatorname{Ind}_{H}^{G_{F}+} \eta_{1, \lambda}^{\text {gal }}$ are calculated as follows:

$$
\begin{aligned}
\operatorname{det} \rho_{f, \lambda} & =\chi_{\ell, \mathrm{cyc}}^{-1} \varepsilon_{+}^{\text {gal }}=\chi_{\ell, \mathrm{cyc}}^{-[\kappa]} \varepsilon_{+}^{f}, \\
\operatorname{det}\left(\operatorname{Ind}_{H}^{G_{F+}} \eta_{1, \lambda}^{\text {gal }}\right) & =\eta_{1, \lambda}^{\text {gal }} \eta_{2, \lambda}^{\text {gal }}=\chi_{\ell, \text { cyc }}^{-n_{1}-n_{2}}\left(\eta_{1, \lambda}^{f} \eta_{2, \lambda}^{f}\right)
\end{aligned}
$$

where $\varepsilon_{+}^{f}$ denotes the finite part of $\varepsilon_{+}^{\text {gal }}$. Since these two 1-dimensional $\lambda$-adic representations must coincide, we obtain the equality $n_{1}+n_{2}=[\kappa]$. Furthermore the equation (A.2) implies that

$$
C(\mathfrak{q} ; f)=\operatorname{Tr} \rho_{f, \lambda}\left(\operatorname{Frob}_{\mathfrak{q}}\right)=\eta_{1, \lambda}^{f}(\mathfrak{Q}) \mathcal{N} \mathfrak{q}^{n_{1}}+\eta_{2, \lambda}^{f}(\mathfrak{Q}) \mathcal{N} \mathfrak{q}^{n_{2}}
$$

holds for each prime ideal $\mathfrak{q}$ of $F^{+}$which splits completely in $F$ as $\mathfrak{q}=\mathfrak{Q Q}^{c}$ (recall that $\chi_{\ell, \text { cyc }}\left(\right.$ Frob $_{\mathfrak{q}}$ ) equals $\left.\mathcal{N q}^{-1}\right)$. By virtue of the Ramanujan-Petersson conjecture

$$
|C(\mathfrak{q} ; f)| \leq 2 \mathcal{N} \mathfrak{q}^{[\kappa] / 2}
$$

established by Brylinski, Labesse [BlRo94, Théorème 3.4.5] and Blasius [Bl06, Theorem 1], both $n_{1}$ and $n_{2}$ must be less than or equal to $[\kappa] / 2$. We thus conclude that, combining this observation with the equality $n_{1}+n_{2}=[\kappa]$, both $n_{1}$ and $n_{2}$ equal $[\kappa] / 2$. More specifically, the restriction $\left.\rho_{f, \lambda}\right|_{H}$ is equivalent to the $\lambda$-adic representation of the form

$$
\left(\begin{array}{cc}
\eta_{1, \lambda}^{f} & 0 \\
0 & \eta_{2, \lambda}^{f}
\end{array}\right) \otimes \chi_{\ell, \mathrm{cyc}}^{-[\kappa] / 2},
$$

which has a finite image in $P G L_{2}\left(\mathbb{Q}_{f, \lambda}\right)$. This contradicts the fact that $\rho_{f, \lambda}$ has an infinite image in $P G L_{2}\left(\mathbb{Q}_{f, \lambda}\right)$ (one readily verifies this fact in the completely same manner as $[\operatorname{Ri} 77$, Theorem (4.3)]), and thus the field $F$ corresponding to $H$ is purely imaginary over $F^{+}$.

Next, we verify that the infinity type $\mu=\sum_{\sigma \in I_{F}} \mu_{\sigma} \sigma$ of $\eta$ is described in terms of the weight of $f$ by setting $\eta=\eta_{1}$. To this end, we take a finite place $\mathfrak{p}$ of $F^{+}$which satisfies the following two properties:

$i)_{\mathfrak{p}}$ the prime ideal $\mathfrak{p}$ is contained in neither of the exceptional set of the strictly compatible system $\left(\rho_{f, \lambda}\right)_{\lambda}$ nor that of $\left(\operatorname{Ind}_{H}^{G_{F}+} \eta_{\lambda}^{\text {gal }}\right)_{\lambda}$;

ii $)_{\mathfrak{p}}$ the unique prime ideal $p \mathbb{Z}$ of $\mathbb{Z}$ lying below $\mathfrak{p}$ splits completely in the extension $F / \mathbb{Q}$.

The existence of such a finite place $\mathfrak{p}$ is guaranteed by Cebotarev's density theorem. Now we fix an algebraic closure $\overline{\mathbb{Q}}_{p}$ of the completion $F_{\mathfrak{p}}^{+}$of $F^{+}$at $\mathfrak{p}$ and an embedding $\iota_{p}: \overline{\mathbb{Q}} \hookrightarrow \overline{\mathbb{Q}}_{p}$. For each embedding $\tau: F^{+} \hookrightarrow \overline{\mathbb{Q}}$ (that is, $\tau$ is an element of $I_{F^{+}}$under the notation of Section 2.1.1), let $\mathfrak{p}_{\tau}$ denote the finite place of $F^{+}$lying above $p \mathbb{Z}$ induced by the composition $\iota_{p} \circ \tau: F^{+} \hookrightarrow \overline{\mathbb{Q}}_{p}$. Note that, due to the condition ii $)_{\mathfrak{p}}$ on $\mathfrak{p}$, the correspondence $\tau \mapsto \mathfrak{p}_{\tau}$ induces a bijection between $I_{F^{+}}$and the set of prime ideals of $F^{+}$lying above $p \mathbb{Z}$. Let $\lambda_{0}$ denote the finite place of $\mathbb{Q}_{f}$ induced by the embedding $\mathbb{Q}_{f} \subset \overline{\mathbb{Q}} \stackrel{\iota_{p}}{\longrightarrow} \overline{\mathbb{Q}}_{p}$ and let us consider the isomorphism

$$
\rho_{f, \lambda_{0}} \cong \operatorname{Ind}_{H}^{G_{F}} \eta_{\lambda_{0}}^{\text {gal }}: G_{F^{+}} \rightarrow G L_{2}\left(\mathbb{Q}_{f, \lambda_{0}}\right)
$$


of the $\lambda_{0}$-adic representations of $G_{F^{+}}$. It is obvious from their constructions that both of $\rho_{f, \lambda_{0}} \mid{ }_{D_{\mathfrak{p}_{\tau}}}$ and $\left.\left(\operatorname{Ind}_{H}^{G_{F}^{+}} \eta_{\lambda_{0}}^{\text {gal }}\right)\right|_{D_{\mathfrak{p}_{\tau}}}$ are Hodge-Tate representation of $D_{\mathfrak{p}_{\tau}}$ for each $\tau$ in $I_{F^{+}}$, and we shall compare the Hodge-Tate weights of these representations. As we have remarked in the paragraphs preceding Remark 2.4, the Hodge type at $\tau$ of the motive $M(f)_{/ F^{+}}$ associated to $f$ is given by $\left\{\left(\kappa_{1, \tau}, \kappa_{2, \tau}\right),\left(\kappa_{2, \tau}, \kappa_{1, \tau}\right)\right\}$, and we thus see that the HodgeTate weights of $\left.\rho_{f, \lambda_{0}}\right|_{D_{\mathfrak{p}_{\tau}}}$ are $\left\{\kappa_{1, \tau}, \kappa_{2, \tau}\right\}$ via the comparison isomorphism in $p$-adic Hodge theory. Now let us study the Hodge-Tate weight of $\left.\left(\operatorname{Ind}_{H}^{G_{F}^{+}} \eta_{\lambda_{0}}^{\text {gal }}\right)\right|_{D_{\mathfrak{p} \tau}}$ for each $\tau$ in $I_{F^{+}}$. Let $w_{\tau}$ denote the unique (complex) place of $F$ lying above the real place of $F^{+}$determined by $\tau$, which is identified with the pair $\left\{\sigma_{\tau, 1}, \sigma_{\tau, 2}\right\}$ of embeddings of $F$ into $\overline{\mathbb{Q}}$ whose restrictions to $F^{+}$coincide with $\tau$. Then the $p$-adic embeddings $\iota_{p} \circ \sigma_{\tau, 1}$ and $\iota_{p} \circ \sigma_{\tau, 2}$ of $F$ induce distinct prime ideals $\mathfrak{P}_{\tau, 1}$ and $\mathfrak{P}_{\tau, 2}$, which are interchanged by the complex conjugation. Recall that the Hodge type at $w_{\tau}$ of the motive $M(\eta)_{/ F}$ associated to the größencharacter $\eta$ of type $\left(A_{0}\right)$ is given by $\left\{\left(\mu_{\sigma_{\tau, 1}}, \mu_{\sigma_{\tau, 2}}\right),\left(\mu_{\sigma_{\tau, 2}}, \mu_{\sigma_{\tau, 1}}\right)\right\}$ (see [B186, Proposition (3.2.3)] or [Sc86, Chapter 1, Section 4] for details). Since $\left.\left(\operatorname{Ind}_{H}^{G_{F}^{+}} \eta_{\lambda_{0}}^{\text {gal }}\right)\right|_{D_{\mathfrak{p}_{\tau}}}$ is isomorphic to $\left.\left.\eta_{\lambda_{0}}^{\text {gal }}\right|_{D_{\mathfrak{P}_{\tau, 1}}} \oplus \eta_{\lambda_{0}}^{\mathrm{gal}}\right|_{D_{\mathfrak{P}_{\tau}, 2}}$, we readily see that the Hodge-Tate weights of $\left.\left(\operatorname{Ind}_{H}^{G_{F}+} \eta_{\lambda_{0}}^{\text {gal }}\right)\right|_{D_{\mathfrak{p}_{\tau}}}$ are $\left\{\mu_{\sigma_{\tau, 1}}, \mu_{\sigma_{\tau, 2}}\right\}$. By comparing the Hodge-Tate weights, we can consider without loss of generality that the two integers $\kappa_{1, \tau}$ and $\kappa_{2, \tau}$ coincide with $\mu_{\sigma_{\tau, 1}}$ and $\mu_{\sigma_{\tau, 2}}$ respectively. Furthermore the inequality $\kappa_{1, \tau}<\kappa_{2, \tau}$ holds for each $\tau$ in $I_{F^{+}}$since we have assumed that the weight $\kappa$ of $f$ was cohomological. Therefore if we set $\Sigma=\left\{\sigma_{\tau, 1}: F \hookrightarrow \overline{\mathbb{Q}} \mid \tau \in I_{F^{+}}\right\}$, it is straightforward to verify that $\Sigma$ is a $p$-ordinary CM type of $F$ with respect to which the infinity type $\mu$ of the größencharacter $\eta$ is admissible. Moreover we readily redescribe $\mu$ in terms of $\kappa$ and $\Sigma$ as in the statement (3).

Finally, we verify that the primitive form $f$ is described as the theta lift of $\eta$. Since the infinity type of $\eta$ is $\Sigma$-admissible, we have the theta lift $\vartheta(\eta)$ of $\eta$ by Proposition-Definition 2.13. The construction of $\eta$ implies that the local $L$-factors of $f$ and $\vartheta(\eta)$ coincide at every prime ideal $\mathfrak{q}$ of $F^{+}$which splits completely in $F$, and we thus conclude that $f$ and $\vartheta(\eta)$ coincides up to a scalar multiple due to the strong multiplicity one theorem for Hilbert modular forms. However both the Fourier coefficient at $\mathfrak{r}_{F^{+}}$of $f$ and that of $\vartheta(\eta)$ equal 1 , and hence the primitive form $f$ exactly coincides with the theta lift $\vartheta(\eta)$ of $\eta$.

As an application of Proposition A.1, we can deduce conditions for the primitive cuspform $\vartheta(\eta)$ with complex multiplication to be nearly $p$-ordinary. In the following proposition we fix a $p$-adic embedding $\iota_{p}: \overline{\mathbb{Q}} \hookrightarrow \overline{\mathbb{Q}}_{p}$.

Proposition A.3. Let $p$ be a prime number and $f$ a primitive Hilbert modular cuspform as above. Then $f$ is nearly p-ordinary (with respect to $\iota_{p}$ ) if and only if there exist a totally imaginary quadratic extension $F / F^{+}$satisfying the ordinarity condition $\left(\operatorname{ord}_{F / F^{+}}\right)$for the prime number $p$, a p-ordinary $C M$ type $\Sigma$ and a $\Sigma$-admissible größencharacter $\eta$ of type $\left(A_{0}\right)$ on $F$ ordinary with respect to $\Sigma$ such that $f$ is obtained as the theta lift $\vartheta(\eta)$ of $\eta$.

Proof. We can easily verify that the condition is sufficient. Indeed, let $F / F^{+}, \Sigma$ and $\eta$ be as in the statement. By the characterisation of the Fourier coefficients of the theta lifts, we readily see that, for every prime ideal $\mathfrak{p}=\mathfrak{P P}^{c}$ lying above $p$, the eigenvalue $C_{0}(\mathfrak{p} ; \vartheta(\eta))$ of the normalised Hecke operator $U_{0}(\mathfrak{p})$ at $\vartheta(\eta)$ equals the summation of $\left\{\mathfrak{p}^{\kappa_{\mu, 1}}\right\}^{-1} \eta^{*}(\mathfrak{P})$ and $\left\{\mathfrak{p}^{\kappa_{\mu, 1}}\right\}^{-1} \eta^{*}\left(\mathfrak{P}^{c}\right)$. Note that the value $\left\{\mathfrak{p}^{\kappa_{\mu, 1}}\right\}^{-1} \eta^{*}(\mathfrak{P})$ does not vanish since $\eta$ is unramified at the unique place $\mathfrak{P}$ in $\Sigma_{p}$ lying above $\mathfrak{p}$ due to the ordinarity of $\eta$ with respect to $\Sigma$. Moreover we readily observe by construction that $\left\{\mathfrak{p}^{\kappa \mu, 1}\right\}^{-1} \eta^{*}(\mathfrak{P})$ has the same $p$-adic valuation with the evaluation $\hat{\eta}_{\mathfrak{P}}\left(\varpi_{\mathfrak{P}}\right)$ of the $\mathfrak{P}$-component of the $p$-adic avatar $\hat{\eta}$ of $\eta$ at a 
uniformiser $\varpi_{\mathfrak{P}}$ of $F_{\mathfrak{P}}$. Therefore $C_{0}(\mathfrak{p} ; \vartheta(\eta))$ is a $p$-adic unit for each $\mathfrak{p}$ and consequently $\vartheta(\eta)$ is nearly $p$-ordinary.

Conversely let $f$ be a nearly $p$-ordinary Hilbert modular cuspform with complex multiplication, and let us take a totally imaginary quadratic extension $F / F^{+}$, a CM type $\Sigma$ and a $\Sigma$-admissible größencharacter $\eta$ of type $\left(A_{0}\right)$ on $F$ as in Proposition A.1 then $f$ is obtained as the theta lift of $\eta$. We denote by $V_{f}$ the Galois representation associated to $f$, which is isomorphic to the induced representation $\operatorname{Ind}_{F}^{F^{+}} \eta^{\text {gal }}$ by Proposition A.1. First assume that there exists a place $\mathfrak{p}$ of $F^{+}$lying above $p$ which does not split in $F$, and let $\mathfrak{P}$ denote the unique place of $F$ above $\mathfrak{p}$. Since we can regard the decomposition group $D_{\mathfrak{P}}$ of $G_{F}$ at $\mathfrak{P}$ as a subgroup of the decomposition group $D_{\mathfrak{p}}$ at $\mathfrak{p}$ of index 2 , we readily identify the restriction of $V_{f}$ to $D_{\mathfrak{p}}$ with the induced representation $\left.\operatorname{Ind}_{D_{\mathfrak{P}}}^{D_{\mathfrak{p}}} \eta^{\text {gal }}\right|_{D_{\mathfrak{P}}}$, which is obviously irreducible. Therefore $\left.V_{f}\right|_{D_{\mathfrak{p}}}$ admits no one-dimensional $D_{\mathfrak{p}}$-subrepresentations. This contradicts Proposition [2.11, and thus all places of $F^{+}$lying above $p$ split in $F$. In other words, the quadratic extension $F / F^{+}$satisfies the condition $\left(\operatorname{ord}_{F / F^{+}}\right)$for $p$.

We next prove that $\Sigma$ is a $p$-ordinary CM type. Let $\mathfrak{p}$ be a place of $F^{+}$lying above $p$, which splits completely in $F$ as $\mathfrak{p}=\mathfrak{P P}^{c}$ by the arguments above. The quadratic equation (2.6) in Proposition 2.11 has two roots $\varpi_{\mathfrak{p}}^{-\kappa_{1, \mathfrak{p}}} \eta^{*}(\mathfrak{P})$ and $\varpi_{\mathfrak{p}}^{-\kappa_{1, \mathfrak{p}}} \eta^{*}\left(\mathfrak{P}^{c}\right)$, one of which is a $p$-adic unit due to the near $p$-ordinarity of $f=\vartheta(\eta)$. We can assume without loss of generality that $\varpi_{\mathfrak{p}}^{-\kappa_{1, \mathfrak{p}}} \eta^{*}(\mathfrak{P})$ is a $p$-adic unit. Define $\Sigma_{\mathfrak{P}}$ and $\Sigma_{\mathfrak{P}}^{c}$ as follows:

$$
\begin{aligned}
& \Sigma_{\mathfrak{P}}=\left\{\sigma \in \Sigma \mid \iota_{p} \circ \sigma \text { induces } \mathfrak{P}\right\}=\left\{\sigma_{1}, \ldots, \sigma_{s}\right\}, \\
& \Sigma_{\mathfrak{P}}^{c}=\left\{\bar{\sigma} \in \Sigma^{c} \mid \iota_{p} \circ \bar{\sigma} \text { induces } \mathfrak{P}\right\}=\left\{\bar{\sigma}_{s+1}, \ldots, \bar{\sigma}_{s+t}\right\} .
\end{aligned}
$$

We shall verify that $\Sigma_{\mathfrak{P}}^{c}$ is empty, or in other words, that $t$ equals 0 . Since $\mathfrak{p}$ splits in $F$, the decomposition group $D_{\mathfrak{p}}$ at $\mathfrak{p}$ is contained in $G_{F}$, and thus the restriction of $V_{f}$ to $D_{\mathfrak{p}}$ is isomorphic to the direct sum of $\left.\eta^{\text {gal }}\right|_{D_{\mathfrak{P}}}$ and $\left.\eta^{\text {gal,c }}\right|_{D_{\mathfrak{P}}}$. Therefore the equation (2.6) in Proposition 2.11 suggests that $\eta^{\text {gal }}\left(\operatorname{Frob}_{\varpi_{\mathfrak{p}}}\right)=\hat{\eta}\left(\varpi_{\mathfrak{P}}\right)$ coincides with $\varpi_{\mathfrak{p}}^{-\kappa_{1, \mathfrak{p}}} \eta^{*}(\mathfrak{P})$. Here we identify $F_{\mathfrak{p}}^{+}$with $F_{\mathfrak{P}}$ and define the uniformiser $\varpi_{\mathfrak{P}}$ of $F_{\mathfrak{P}}$ as $\varpi_{\mathfrak{p}}$ via this identification. By the definition of the $p$-adic avatar (2.2), we have

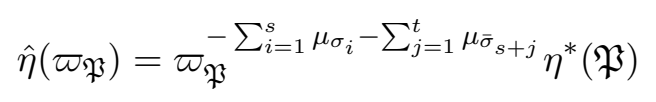

where we denote by $\mu=\sum_{\sigma \in \Sigma}\left(\mu_{\sigma} \sigma+\mu_{\bar{\sigma}} \bar{\sigma}\right)$ the infinity type of $\eta$. On the other hand, since $\kappa_{1}=\kappa_{\mu, 1}$ equals $\left.\sum_{\sigma \in \Sigma} \mu_{\sigma} \sigma\right|_{F^{+}}$by the characterisation of the weight of the theta lift (see (2.8) for details), we have

$$
\varpi_{\mathfrak{p}}^{-\kappa_{1, \mathfrak{p}}} \eta^{*}(\mathfrak{P})=\varpi_{\mathfrak{P}}^{-\sum_{i=1}^{s+t} \mu_{i}} \eta^{*}(\mathfrak{P}) .
$$

Comparing the exponent of $\varpi_{\mathfrak{P}}$ in the equations (A.3) and (A.4), we obtain the equality

$$
\sum_{j=1}^{t}\left(\mu_{\bar{\sigma}_{s+j}}-\mu_{\sigma_{s+j}}\right)=0 .
$$

The $\Sigma$-admissibility of $\eta$ implies the inequality $\mu_{\bar{\sigma}_{s+j}}>\mu_{\sigma_{s+j}}$ for each $j$, which forces $t$ to be 0 . Therefore the CM type $\Sigma$ is indeed a $p$-ordinary CM type.

Finally since $\varpi_{\mathfrak{p}}^{-\kappa_{1, \mathfrak{p}}} \eta^{*}(\mathfrak{P})$ is a $p$-unit for every place $\mathfrak{p}=\mathfrak{P P}^{c}$ of $F^{+}$lying above $p$, the value $\eta^{*}(\mathfrak{P})$ does not vanish for every $\mathfrak{P}$ in $\Sigma_{p}$. This implies that the größencharacter $\eta$ is ordinary with respect to $\Sigma$. 


\section{ApPEndix B. COMPARISON OF THE GLOBAL AND LOCAL DUALITY PAIRINGS}

In this appendix we provide the proof of Proposition 3.40. We take over the same setting and use the same notation as in Section 3.4.5. Firstly we recall that, as is explained in the preceding paragraphs of [Gr10, Proposition 3.3.1], the Pontrjagin dual of the cokernel of the global-to-local morphism $\phi_{\mathcal{L}_{\mathrm{str}}^{*(j-1)}}$ is identified with the image of the global-to-local map

$$
\left.\operatorname{Res}_{(j-1)}^{1}\right|_{\operatorname{Sel}_{\mathcal{A}_{\eta,(j-1)}}^{\Sigma, \mathrm{str}}}: \operatorname{Sel}_{\mathcal{A}_{\eta,(j-1)}^{\Sigma, \mathrm{str}}} \rightarrow L_{\mathrm{str}}\left(F, \mathcal{A}_{\eta,(j-1)}\right) \quad\left(\subset \prod_{v \in S} H^{1}\left(F_{v}, \mathcal{A}_{\eta,(j-1)}\right)\right)
$$

via the local Tate duality isomorphism

$$
\Phi_{\text {local }}=\left(\Phi_{\text {local }, v}\right)_{v \in S}: \prod_{v \in S} H^{1}\left(F_{v}, \mathcal{A}_{\eta,(j-1)}\right)^{\vee} \stackrel{\sim}{\rightarrow} \prod_{v \in S} H_{\text {cts }}^{1}\left(F_{v}, \mathcal{T}_{\eta,(j-1)}^{*}\right) .
$$

In other words, the summation of the local Tate pairing

$$
\langle,\rangle_{\text {local }}=\sum_{v \in S}\langle,\rangle_{v}: \prod_{v \in S} H^{1}\left(F_{v}, \mathcal{A}_{\eta,(j-1)}\right) \times \prod_{v \in S} H_{\mathrm{cts}}^{1}\left(F_{v}, \mathcal{T}_{\eta,(j-1)}^{*}\right) \rightarrow \mathbb{Q}_{p} / \mathbb{Z}_{p}
$$

induces a perfect pairing

$$
\langle,\rangle_{\text {local }}: \operatorname{Im}\left(\left.\operatorname{Res}_{(j-1)}^{1}\right|_{\operatorname{Sel}_{\mathcal{A}_{\eta,(j-1)}^{\Sigma, \text { str }}}}\right) \times \operatorname{Coker}\left(\phi_{\mathcal{L}_{\text {str }}^{*,(j-1)}}\right) \rightarrow \mathbb{Q}_{p} / \mathbb{Z}_{p}
$$

for which we use the same symbol $\langle,\rangle_{\text {local }}$ to simplify the notation. Since the module $\mathcal{C}^{(j-1)}$ introduced in the diagram (3.28) is defined as the cokernel of the multiplication of $x_{j}$ on $\operatorname{Coker}\left(\phi_{\mathcal{L}_{\mathrm{str}}^{*,(j-1)}}\right)$, the perfect pairing $\langle,\rangle_{\text {local }}$ above also induces a perfect pairing

$$
\langle,\rangle_{\text {local }}: \mathcal{K}^{(j-1)} \times \mathcal{C}^{(j-1)} \rightarrow \mathbb{Q}_{p} / \mathbb{Z}_{p}
$$

where $\mathcal{K}^{(j-1)}$ is defined as

$$
\mathcal{K}^{(j-1)}:=\operatorname{Ker}\left[\operatorname{Im}\left(\left.\operatorname{Res}_{(j-1)}^{1}\right|_{\operatorname{Sel}_{\mathcal{A}_{\eta,(j-1)}^{\Sigma, \text { str }}}}\right) \stackrel{\times x_{j}}{\longrightarrow} \operatorname{Im}\left(\left.\operatorname{Res}_{(j-1)}^{1}\right|_{\operatorname{Sel}_{\mathcal{A}_{\eta,(j-1)}, \mathrm{str}}}\right)\right] .
$$

Now consider the commutative diagram

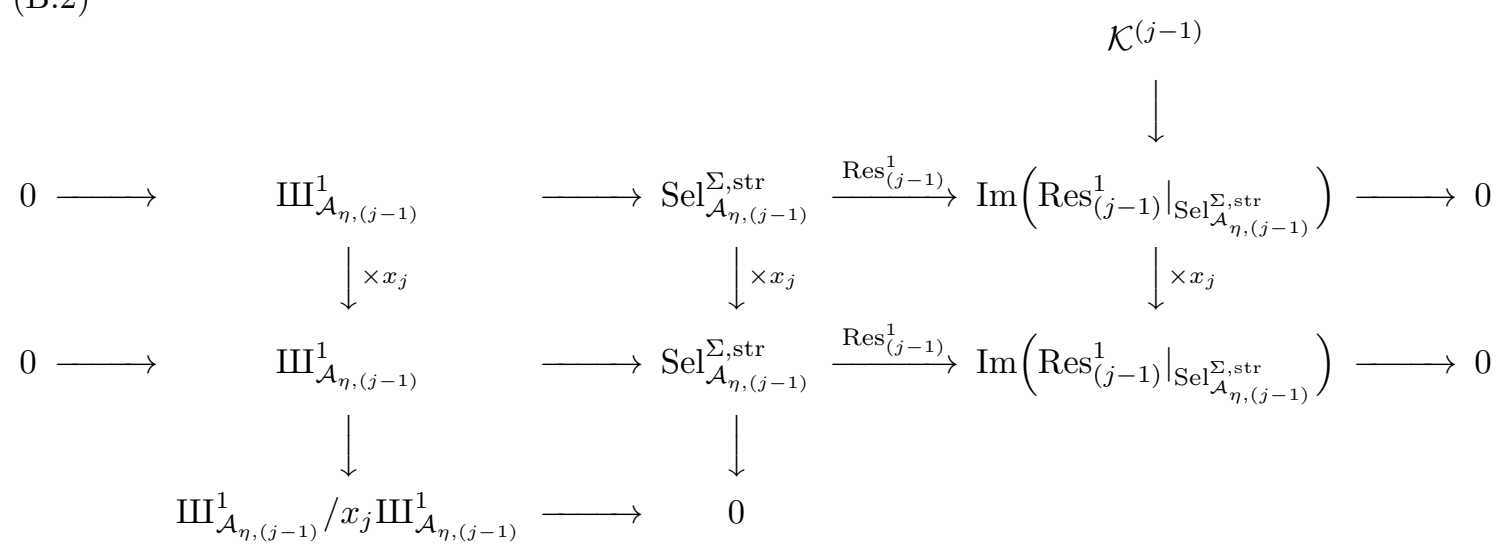

whose rows are exact by the definition of the $S$-fine Selmer group $\amalg_{\mathcal{A}_{\eta,(j-1)}}^{1}$. We denote by

$$
\delta_{1}: \mathcal{K}^{(j-1)} \rightarrow \amalg_{\mathcal{A}_{\eta,(j-1)}}^{1} / x_{j} \amalg_{\mathcal{A}_{\eta,(j-1)}}^{1}
$$

the connecting homomorphism associated to the diagram (B.2) through the snake lemma. 
The diagram (B.2) is obtained as the Pontrjagin dual of the diagram of (3.24), and we thus observe that the connecting homomorphism $\tilde{\delta}_{1}^{\vee}$ in the diagram (3.28) is obtained as the composition

$$
\left(\amalg_{\mathcal{A}_{\eta,(j-1)}}^{1}\right)^{\vee}\left[x_{j}\right] \stackrel{\delta_{1}^{\vee}}{\longrightarrow}\left(\mathcal{K}^{(j-1)}\right)^{\vee} \stackrel{\Phi_{\text {local }}}{\sim} \mathcal{C}^{(j-1)}
$$

where $\delta_{1}^{\vee}$ denotes the dual morphism of $\delta_{1}$ and $\Phi_{\text {local }}$ is the isomorphism induced by the perfect pairing $\langle,\rangle_{\text {local }}$. By construction the local Tate duality map $\Phi_{\text {local }}$ isomorphically sends the image of $\delta_{1}^{\vee}$ onto that of $\tilde{\delta}_{1}^{\vee}$, or in other words, the perfect pairing $\langle,\rangle_{\text {local }}$ induces a perfect pairing

$$
\langle,\rangle_{\text {local }}: \operatorname{Coim}\left(\delta_{1}\right) \times \operatorname{Im}\left(\tilde{\delta}_{1}^{\vee}\right) \rightarrow \mathbb{Q}_{p} / \mathbb{Z}_{p}
$$

which makes the following diagram commutative:

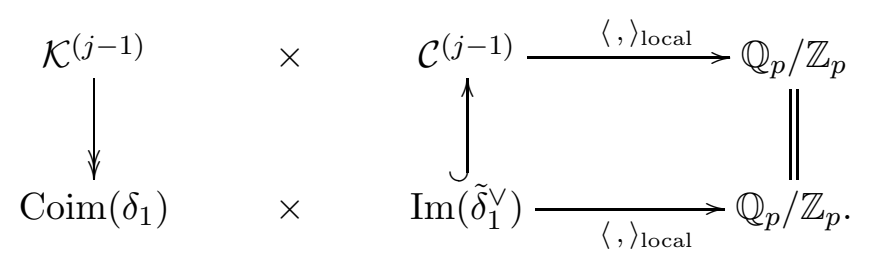

Recall that we have defined the (injective) morphism $\delta_{2}: \operatorname{Im}\left(\tilde{\delta}_{1}^{\vee}\right) \rightarrow \overline{\mathcal{H}}_{j}^{2, *}$ as the connecting homomorphism associated to the diagram (3.41) via the snake lemma. See the paragraph preceding the short exact sequences (3.42) and (3.43) for the definition of the module $\overline{\mathcal{H}}_{j}^{2, *}$. Let $\amalg_{\mathcal{T}_{\eta,(j-1)}^{*}}^{2}$ denote the kernel of the local-to-global morphism

$$
\operatorname{Res}_{(j-1)}^{2, *}: H_{(j-1)}^{2, *} \rightarrow \prod_{v \in S} H_{\mathrm{cts}}^{2}\left(F_{v}, \mathcal{T}_{\eta,(j-1)}^{*}\right)
$$

Combining the short exact sequence defining $\amalg_{\mathcal{T}_{\eta,(j-1)}^{*}}^{2}$ and the sequence (3.43) with $\operatorname{Im}\left(\tilde{\delta}_{1}^{\vee}\right)$ replaced by $\operatorname{Im}\left(\delta_{2}\right)$, we obtain the following commutative diagram with exact rows:

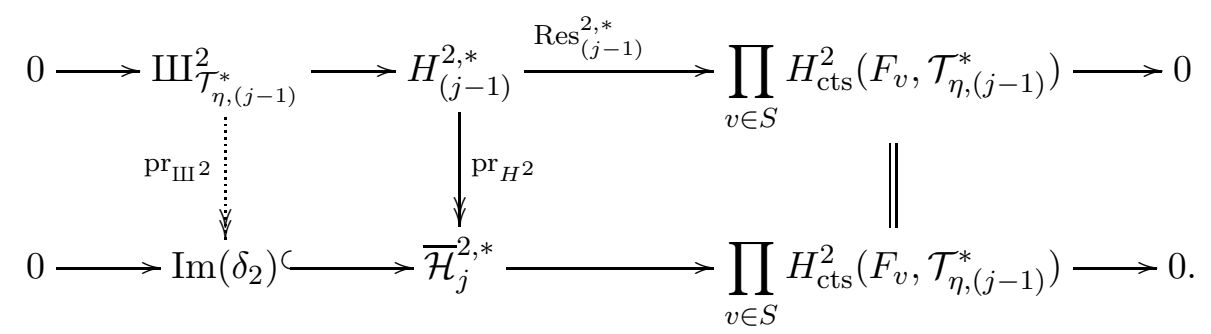

We readily observe that the middle vertical morphism $\mathrm{pr}_{H^{2}}$, which is defined in (3.42), induces a surjection $\mathrm{pr}_{\amalg^{2}}: \amalg_{\mathcal{T}_{\eta,(j-1)}^{*}}^{2} \rightarrow \operatorname{Im}\left(\delta_{2}\right)$. Note that the right commutative square of the diagram above is the same as that of the diagram (3.45). The rest of this appendix is devoted to the verification of the following proposition.

Proposition B.1. Let

$$
\langle,\rangle_{\mathrm{PT}}: \amalg_{\mathcal{A}_{\eta,(j-1)}}^{1} / x_{j} \amalg_{\mathcal{A}_{\eta,(j-1)}}^{1} \times \amalg_{\mathcal{T}_{\eta,(j-1)}^{*}}^{2}\left[x_{j}\right] \rightarrow \mathbb{Q}_{p} / \mathbb{Z}_{p}
$$

be the perfect pairing induced from the Poitou-Tate pairing $\amalg_{\mathcal{A}_{\eta,(j-1)}}^{1} \times \amalg_{\mathcal{T}_{\eta,(j-1)}^{*}}^{2} \rightarrow \mathbb{Q}_{p} / \mathbb{Z}_{p}$. Then the equality

$$
\left\langle\left(s_{v}\right)_{v \in S},\left(t_{v}\right)_{v \in S}\right\rangle_{\text {local }}=\left\langle\delta_{1}\left(\left(s_{v}\right)_{v \in S}\right), \delta_{2}\left(\left(t_{v}\right)_{v \in S}\right)^{\sim}\right\rangle_{\mathrm{PT}}
$$


holds for arbitrary elements $\left(s_{v}\right)_{v \in S}$ and $\left(t_{v}\right)_{v \in S}$ in $\operatorname{Coim}\left(\delta_{1}\right)$ and $\operatorname{Im}\left(\tilde{\delta}_{1}^{\vee}\right)$ respectively, where $\delta_{2}\left(\left(t_{v}\right)_{v \in S}\right)^{\sim}$ denotes an arbitrary element of $\amalg^{2}\left(F, S, \mathcal{T}_{\eta,(j-1)}^{*}\right)\left[x_{j}\right]$ which is sent to $\delta_{2}\left(\left(t_{v}\right)_{v \in S}\right)$ by the map $\mathrm{pr}_{\amalg^{2}}$ introduced above. In particular the perfect pairing $\langle,\rangle_{\mathrm{PT}}$ induces a pairing

$$
\amalg_{\mathcal{A}_{\eta,(j-1)}}^{1} / x_{j} \amalg_{\mathcal{A}_{\eta,(j-1)}}^{1} \times \operatorname{Im}\left(\delta_{2}\right) \longrightarrow \mathbb{Q}_{p} / \mathbb{Z}_{p}
$$

which makes the following diagram commutative:

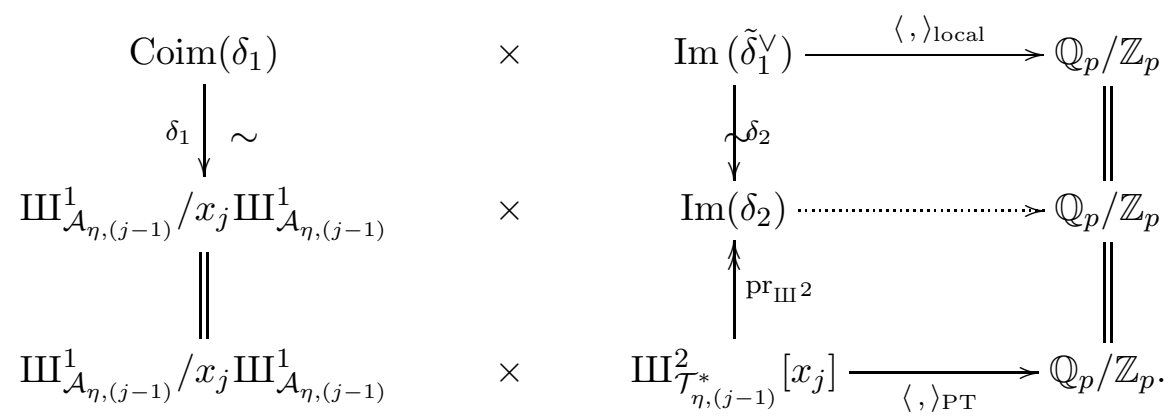

We readily observe that Proposition 3.40 is a direct consequence of Proposition B.1. As we remarked after Proposition 3.40, the right square of the diagram in Proposition 3.40 is obvious and we only need to check the commutativity of the left square of the diagram in Proposition 3.40, Let $f: \amalg_{\mathcal{A}_{\eta,(j-1)}}^{1} \rightarrow \mathbb{Q}_{p} / \mathbb{Z}_{p}$ be an arbitrary element of $\left(\amalg_{\mathcal{A}_{\eta,(j-1)}}^{1}\right)^{\vee}\left[x_{j}\right]$ and $a$ an arbitrary element of $\amalg_{\mathcal{A}_{\eta,(j-1)}}^{1} / x_{j} \amalg_{\mathcal{A}_{\eta,(j-1)}}^{1}$. Then there exists a unique element $b$ of $\operatorname{Coim}\left(\delta_{1}\right)$ satisfying $\delta_{1}(b)=a$. By definition the duality isomorphisms $\Phi_{\text {local }}$ and $\Phi_{\mathrm{PT}}$ are characterised by the relations

$$
\delta_{1}^{\vee}(f)(b)=\left\langle b, \Phi_{\text {local }}\left(\delta_{1}^{\vee}(f)\right)\right\rangle_{\text {local }}, \quad \quad f(a)=\left\langle a, \Phi_{\mathrm{PT}}(f)\right\rangle_{\mathrm{PT}} .
$$

Proposition B.1 thus provides the following equality:

$$
\begin{aligned}
\left\langle a, \Phi_{\mathrm{PT}}(f)\right\rangle_{\mathrm{PT}} & =f(a)=f\left(\delta_{1}(b)\right)=\delta_{1}^{\vee}(f)(b) \\
& =\left\langle b, \Phi_{\text {local }}\left(\delta_{1}^{\vee}(f)\right)\right\rangle_{\text {local }} \\
& =\left\langle\delta_{1}(b), \delta_{2}\left(\Phi_{\text {local }}\left(\delta_{1}^{\vee}(f)\right)\right)^{\sim}\right\rangle_{\mathrm{PT}} \quad \text { (here we apply Proposition B.1) } \\
& =\left\langle a, \delta_{2}\left(\Phi_{\text {local }}\left(\delta_{1}^{\vee}(f)\right)\right)^{\sim}\right\rangle_{\mathrm{PT}} .
\end{aligned}
$$

The perfectness of the Poitou-Tate pairing thus implies the equality

$$
\Phi_{\mathrm{PT}}(f)=\delta_{2}\left(\Phi_{\text {local }}\left(\delta_{1}^{\vee}(f)\right)\right)^{\sim} .
$$

Since the connecting homomorphism $\boldsymbol{\delta}_{\text {snake }}$ appearing in Proposition 3.40 is decomposed as $\boldsymbol{\delta}_{\text {snake }}=\delta_{2} \circ \Phi_{\text {local }} \circ \delta_{1}^{\vee}$ due to (3.43) and (B.3), we finally deduce the desired equality

$$
\operatorname{pr}_{H^{2}} \circ \Phi_{\mathrm{PT}}(f)=\delta_{2}\left(\Phi_{\text {local }}\left(\delta_{1}^{\vee}(f)\right)\right)=\boldsymbol{\delta}_{\text {snake }}(f)
$$

by applying $\operatorname{pr}_{\amalg^{2}}\left(=\operatorname{pr}_{H^{2}}\right)$ to the both sides of (B.7). The final equality shows the commutativity of the left square of the diagram in Proposition 3.40 .

The proof of Proposition B.1 requires a little lengthy computation. We first calculate the cohomology classes $\delta_{1}\left(\left(s_{v}\right)_{v \in S}\right)$ and $\delta_{2}\left(\left(t_{v}\right)_{v \in S}\right)$ explicitly, and then check that the value $\left\langle\delta_{1}\left(\left(s_{v}\right)_{v \in S}\right), \delta_{2}\left(\left(t_{v}\right)_{v \in S}\right)^{\sim}\right\rangle_{\mathrm{PT}}$ coincides with $\left\langle\left(s_{v}\right)_{v \in S},\left(t_{v}\right)_{v \in S}\right\rangle_{\text {local }}$ by a direct computation. In order to estimate the value $\left\langle\delta_{1}\left(\left(s_{v}\right)_{v \in S}\right), \delta_{2}\left(\left(t_{v}\right)_{v \in S}\right)^{\sim}\right\rangle_{\mathrm{PT}}$, we utilise the explicit 
formula of the Poitou-Tate pairing $\langle,\rangle_{\mathrm{PT}}: \amalg_{\mathcal{A}_{\eta,(j-1)}}^{1} \times \amalg_{\mathcal{T}_{\eta,(j-1)}^{*}}^{2} \rightarrow \mathbb{Q}_{p} / \mathbb{Z}_{p}$, which we recall below (see [Tat63, Section 3] for details1). In the rest of the article, we denote by $C^{\bullet}(G, M)$ the standard cochain complex of a continuous $G$-module $M$ over a profinite group $G$, and by $d_{M}$ (or just $d$ if it is clear from the context) its coboundary homomorphism. Let $[f]$ be an element of $\amalg_{\mathcal{T}_{\eta,(j-1)}^{*}}^{2}$ represented by a continuous 2-cocycle $f$ in $C^{2}\left(\operatorname{Gal}\left(F_{S} / F\right), \mathcal{T}_{\eta,(j-1)}^{*}\right)$, and $\left[f^{\prime}\right]$ an element of $\amalg_{\mathcal{A}_{\eta,(j-1)}}^{1}$ represented by a continuous 1-cocycle $f^{\prime}$ in $C^{1}\left(\operatorname{Gal}\left(F_{S} / F\right), \mathcal{A}_{\eta,(j-1)}\right)$. For each $v$ in $S$, we denote by $f_{v}$ the restriction of $f$ to the decomposition group $D_{v}$ at $v$. Since $[f]$ is locally trivial at $v$, there exists a 1-cochain $g_{v}$ in $C^{1}\left(D_{v}, \mathcal{T}_{\eta,(j-1)}^{*}\right)$ satisfying $d g_{v}=f_{v}$. Recall that $F$ has no real places, which implies the triviality of the global cohomology group $H^{3}\left(F_{S} / F, \mu_{p} \infty\right)$ (see [NSW00, (8.6.10) (ii)] for example). Thus there exists a 2-cochain $h$ in $C^{2}\left(\operatorname{Gal}\left(F_{S} / F\right), \mu^{p^{\infty}}\right)$ satisfying $d h=f^{\prime} \cup f$. Then the value $\left\langle\left[f^{\prime}\right],[f]\right\rangle_{\mathrm{PT}}$ is explicitly calculated as

$$
\left\langle\left[f^{\prime}\right],[f]\right\rangle_{\mathrm{PT}}=\sum_{v \in S}\left\{-f_{v}^{\prime} \cup g_{v}-h_{v}\right\}_{v}
$$

where, for each $v$ in $S, h_{v}$ denotes the restriction of $h$ to the decomposition subgroup $D_{v}$, and $\{\cdot\}_{v}: H^{2}\left(F_{v}, \mu_{p^{\infty}}\right) \stackrel{\sim}{\rightarrow} \mathbb{Q}_{p} / \mathbb{Z}_{p}$ is the invariant isomorphism at $v$.

Now let us calculate $\delta_{1}\left(\left(s_{v}\right)_{v \in S}\right)$ and $\delta_{2}\left(\left(t_{v}\right)_{v \in S}\right)$.

Calculation of $\delta_{1}\left(\left(s_{v}\right)_{v \in S}\right)$. Let $\left(s_{v}\right)_{v \in S}$ be an arbitrary element of Coim $\left(\delta_{1}\right)$. There exists an element $[z]$ of $\operatorname{Sel}_{\mathcal{A}_{\eta,(j-1)}^{\Sigma, \text { str }}}$, represented by a 1-cocycle $z$ in $C^{1}\left(\operatorname{Gal}\left(F_{S} / F\right), \mathcal{A}_{\eta,(j-1)}\right)$, which is sent to $\left(s_{v}\right)_{v \in S}$ by $\operatorname{Res}_{(j-1)}^{1}$. Then, following the definition of the connecting homomorphism $\delta_{1}$ given at the diagram $(\overline{B .2})$, the evaluation of $\delta_{2}$ at $\left(s_{v}\right)_{v \in S}$ is calculated as

$$
\delta_{1}\left(\left(s_{v}\right)_{v \in S}\right)=\left[x_{j} z\right] \quad \bmod x_{j} \amalg_{\mathcal{A}_{\eta,(j-1)}}^{1} .
$$

For each $v$ in $S$, we denote by $z_{v}$ the restriction of the 1-cocycle $z$ to the decomposition group $D_{v}$ at $v$.

Calculation of $\delta_{2}\left(\left(t_{v}\right)_{v \in S}\right)$. Let $\left(t_{v}\right)_{v \in S}$ be an arbitrary element of $\operatorname{Im}\left(\tilde{\delta}_{1}^{\vee}\right)$. We calculate $\delta_{2}\left(\left(t_{v}\right)_{v \in S}\right)$ by the diagram chasing on (3.37) (or on (3.41)). For each $v$ in $S$, take a cocycle $w_{v}$ of $C^{1}\left(F_{v}, \mathcal{T}_{\eta,(j-1)}^{*}\right)$ so that the element represented by the cohomology class $\left(\left[w_{v}\right]\right)_{v \in S}$ in $Q_{(j-1)}^{*}$ is sent to $\left(t_{v}\right)_{v \in S}$ under the natural surjection $Q_{(j-1)}^{*} / x_{j} Q_{(j-1)}^{*} \rightarrow \mathcal{C}^{(j-1)}$. Here we use the same symbol $\left(t_{v}\right)_{v \in S}$ for its image in $\mathcal{C}^{(j-1)}$. Denoting by $\bar{w}_{v}$ the image of $w_{v}$ under the surjection $C^{1}\left(F_{v}, \mathcal{T}_{\eta,(j-1)}^{*}\right) \rightarrow C^{1}\left(F_{v}, \mathcal{T}_{\eta,(j)}^{*}\right)$, we have $\pi_{j}^{\mathcal{Q}}\left(\left(\left[w_{v}\right]\right)_{v \in S}\right)=\left(\left[\bar{w}_{v}\right]\right)_{v \in S}$. Then the diagram (3.41) implies that there exists a unique element $\varrho$ of $\operatorname{Coim}\left(\phi_{\mathcal{L}_{\mathrm{str}}^{*(j)}}\right)$ satisfying $\phi_{\mathcal{L}_{\text {str }}^{*,(j)}}(\varrho)=\left(\left[\bar{w}_{v}\right]\right)_{v \in S}$. The image of $\varrho$ in $\overline{\mathcal{H}}_{j}^{2, *}$ is none other than $\delta_{2}\left(\left(t_{v}\right)_{v \in S}\right)$ by definition.

We do a little more precise computation. Let us take an arbitrary lift $\varrho$ of $\varrho$ with respect to the canonical surjection $H_{(j)}^{1, *} \rightarrow \operatorname{Coim}\left(\phi_{\mathcal{L}_{\text {str }}^{*,(j)}}\right)$, which is represented by a 1-cocycle $\tilde{w}$ in $C^{1}\left(\operatorname{Gal}\left(F_{S} / F\right), \mathcal{T}_{\eta,(j)}^{*}\right)$. Then the diagram (‥40) implies that the image of $\delta_{(j)}^{1}([\tilde{w}])$ in $\overline{\mathcal{H}}_{j}^{2, *}$ coincides with $\delta_{2}\left(\left(t_{v}\right)_{v \in S}\right)$. In other words, $\delta_{(j)}^{1}([\tilde{w}])$ is regarded as a lift $\delta_{2}\left(\left(t_{v}\right)_{v \in S}\right)^{\sim}$ of $\delta_{2}\left(\left(t_{v}\right)_{v_{\in} S}\right)$. The element $\delta_{(j)}^{1}([\tilde{w}])$ is, however, calculated in the usual manner; namely, if

\footnotetext{
${ }^{1}$ Indeed the explicit description of the Poitou-Tate pairing is proposed only for finite Galois modules in Tat63. and other literatures, but it is straightforward to justify the same description under our settings by the standard limit argument based upon Tate's theorem on the inverse limits of Galois cohomology groups.
} 
we take a 1-cochain (not necessarily a 1-cocycle) $\hat{w}$ of $C^{1}\left(\operatorname{Gal}\left(F_{S} / F\right), \mathcal{T}_{\eta,(j-1)}^{*}\right)$ which is sent to $\tilde{w}$ under the natural surjection $C^{1}\left(\operatorname{Gal}\left(F_{S} / F\right), \mathcal{T}_{\eta,(j-1)}^{*}\right) \rightarrow C^{1}\left(\operatorname{Gal}\left(F_{S} / F\right), \mathcal{T}_{\eta,(j)}^{*}\right)$, the image $\delta_{(j)}^{1}([\tilde{w}])$ of $[\tilde{w}]$ coincides with $\left[x_{j}^{-1} d \hat{w}\right]$. Note that the lift $\delta_{2}\left(\left(t_{v}\right)_{v \in S}\right)^{\sim}$ of $\delta_{2}\left(\left(t_{v}\right)_{v \in S}\right)$ is determined uniquely modulo $\operatorname{Sel}_{\mathcal{T}_{\eta,(j)}^{*}}^{*, \mathrm{st}}$, but this ambiguity corresponds to that of the choices of lifts $\tilde{\varrho}=[\tilde{w}]$ of $\varrho$. Therefore each $\delta_{2}\left(\left(t_{v}\right)_{v \in S}\right)^{\sim}$ is obtained in this procedure, or more specifically, we obtain an explicit description of an arbitrary lift $\delta_{2}\left(\left(t_{v}\right)_{v \in S}\right)^{\sim}$ of $\delta_{2}\left(\left(t_{v}\right)_{v \in S}\right)$ as $\left[x_{j}^{-1} d \hat{w}\right]$, for an appropriate $\hat{w}$ as above.

Replacement of local cochains. As we have obtained explicit descriptions of $\delta_{1}\left(\left(s_{v}\right)_{v \in S}\right)$ and $\delta_{2}\left(\left(t_{v}\right)_{v \in S}\right)^{\sim}$, we evaluate these elements under the Poitou-Tate pairing:

$$
\left\langle\delta_{1}\left(\left(s_{v}\right)_{v \in S}\right), \delta_{2}\left(\left(t_{v}\right)_{v \in S}\right)^{\sim}\right\rangle_{\mathrm{PT}}=\left\langle\left[x_{j} z\right],\left[x_{j}^{-1} d \hat{w}\right]\right\rangle_{\mathrm{PT}} .
$$

In order to apply the explicit formula (B.8) to (B.9), we need to find a 1-cochain $\check{w}_{v}$ of $C^{1}\left(F_{v}, \mathcal{T}_{\eta,(j-1)}^{*}\right)$ satisfying the equality $d \check{w}_{v}=x_{j}^{-1} d \hat{w}_{v}$ of cocycles for each $v$ in $S$, where $\hat{w}_{v}$ denotes the restriction of $\hat{w}$ to the decomposition group $D_{v}$. In order to find such a nice 1-cochain $\check{w}_{v}$, we first study the relation between $\hat{w}_{v}$ and $w_{v}$, the cocycle which we first took in the computation of $\delta_{2}\left(\left(t_{v}\right)_{v \in S}\right)$ above.

Lemma B.2. For each $v$ in $S$, there exist 1-cochains $\ell_{v}$ and $c_{v}$ in $C^{1}\left(F_{v}, \mathcal{T}_{\eta,(j-1)}^{*}\right)$ satisfying the following two properties.

(1) the image of $\ell_{v}$ under the canonical surjection $C^{1}\left(F_{v}, \mathcal{T}_{\eta,(j-1)}^{*}\right) \rightarrow C^{1}\left(F_{v}, \mathcal{T}_{\eta,(j)}^{*}\right)$ is a 1-cocycle representing a cohomology class contained in $L_{\mathrm{str}}\left(F_{v}, \mathcal{T}_{\eta,(j)}^{*}\right)$;

(2) the cochains $\hat{w}_{v}$ and $w_{v}+\ell_{v}+x_{j} c_{v}$ coincide modulo coboundaries.

Proof. Let $\tilde{w}_{v}$ denote the restriction of the cocycle $\tilde{w}$ to the decomposition group $D_{v}$. Then by construction, $\tilde{w}_{v}$ satisfies the equation

$$
\left(\left[\tilde{w}_{v}\right]\right)_{v \in S}\left(=\phi_{\mathcal{L}_{\mathrm{str}}^{*,(j)}}([\tilde{w}])\right)=\left(\left[\bar{w}_{v}\right]\right)_{v \in S}
$$

in $Q_{(j)}^{*}$, and hence there exists a 1 -cocycle $\bar{\ell}_{v}$ of $C^{1}\left(F_{v}, \mathcal{T}_{\eta,(j)}^{*}\right)$, representing a cohomology class contained in $L_{\mathrm{str}}^{*}\left(F_{v}, \mathcal{T}_{\eta,(j)}^{*}\right)$, such that $\tilde{w}_{v}$ coincides with $\bar{w}_{v}+\bar{\ell}_{v}$ modulo coboundaries. Let $\ell_{v}$ be an arbitrary 1-cochain in $C^{1}\left(F_{v}, \mathcal{T}_{\eta,(j-1)}^{*}\right)$ which is sent to $\bar{\ell}_{v}$ under the natural surjection $C^{1}\left(F_{v}, \mathcal{T}_{\eta,(j-1)}^{*}\right) \rightarrow C^{1}\left(F_{v}, \mathcal{T}_{\eta,(j)}^{*}\right)$. Again by construction, $\hat{w}_{v}$ and $w_{v}+\ell_{v}$ has the same image $\bar{w}_{v}+\bar{\ell}_{v}$ in $C^{1}\left(F_{v}, \mathcal{T}_{\eta,(j)}^{*}\right)$. We now readily verify the existence of a cochain $c_{v}$ satisfying the claim due to the natural exact sequence

$$
C^{1} / B^{1}\left(F_{v}, \mathcal{T}_{\eta,(j-1)}^{*}\right) \stackrel{\times x_{j}}{\longrightarrow} C^{1} / B^{1}\left(F_{v}, \mathcal{T}_{\eta,(j-1)}^{*}\right) \rightarrow C^{1} / B^{1}\left(F_{v}, \mathcal{T}_{\eta,(j)}^{*}\right) \rightarrow 0 .
$$

Here $C^{1} / B^{1}\left(F_{v}, \mathcal{T}_{\eta,(k)}^{*}\right)$ denotes the quotient of $C^{1}\left(F_{v}, \mathcal{T}_{\eta,(k)}^{*}\right)$ with respect to its submodule consisting of all coboundaries.

Lemma B.2 enables us to replace $\hat{w}_{v}$ by $w_{v}+\ell_{v}+x_{j} c_{v}$ in the computation of the PoitouTate pairing (B.9) .

Computation of the Poitou-Tate pairing. We are ready to calculate the evaluation of the Poitou-Tate pairing (B.9). On the one hand, we readily observe that the local cocycle $x_{j}^{-1} d \hat{w}_{v}$ is redescribed as

$$
x_{j}^{-1} d \hat{w}_{v}=x_{j}^{-1} d\left(w_{v}+\ell_{v}+x_{j} c_{v}\right)=x_{j}^{-1} d \ell_{v}+d c_{v}
$$


up to coboundaries by virtue of Lemma B.2. On the other hand, the cohomology class $\left(\left[x_{j}^{-1} d \hat{w}_{v}\right]\right)_{v \in S}$ is the restriction of the element $\delta_{2}\left(\left(t_{v}\right)_{v \in S}\right)^{\sim}=\left[x_{j}^{-1} d \hat{w}\right]$, which is an element of $\amalg_{\mathcal{T}_{\eta,(j-1)}^{*}}^{2}$ by construction. This observation implies that each $x_{j}^{-1} d \hat{w}_{v}=x_{j}^{-1} d \ell_{v}+d c_{v}$ itself is a coboundary. Let us take for every $v$ in $S$ a 1-cochain $\lambda_{v}$ in $C^{1}\left(F_{v}, \mathcal{T}_{\eta,(j)}^{*}\right)$ satisfying $x_{j}^{-1} d \ell_{v}=d \lambda_{v}$ so that $x_{j}^{-1} d \hat{w}_{v}=d\left(\lambda_{v}+c_{v}\right)$ holds up to coboundaries. Moreover the equation

$$
x_{j} z \cup x_{j}^{-1} d \hat{w}=z \cup d \hat{w}=-d(z \cup \hat{w})
$$

holds since $z$ is a 1-cocycle. Applying the explicit formula (B.8) for $f=x_{j}^{-1} d \hat{w}, f^{\prime}=x_{j} z$, $g_{v}=\lambda_{v}+c_{v}$ and $h_{v}=-(z \cup \hat{w})_{v}=-z_{v} \cup \hat{w}_{v}$, we calculate the value of the Poitou-Tate pairing $\left\langle\delta_{1}\left(\left(s_{v}\right)_{v \in S}\right), \delta_{2}\left(\left(t_{v}\right)_{v \in S}\right)^{\sim}\right\rangle_{\mathrm{PT}}$ as follows:

$$
\begin{aligned}
\left\langle\delta_{1}\left(\left(s_{v}\right)_{v \in S}\right), \delta_{2}\left(\left(t_{v}\right)_{v \in S}\right)^{\sim}\right\rangle_{\mathrm{PT}} & =\sum_{v \in S}\left\{-\left[x_{j} z_{v}\right] \cup\left[\lambda_{v}+c_{v}\right]-\left(-z_{v} \cup \hat{w}_{v}\right)\right\}_{v} \\
& =\sum_{v \in S}\left\{-\left[z_{v}\right] \cup x_{j}\left[\lambda_{v}+c_{v}\right]+\left[z_{v}\right] \cup\left[w_{v}+\ell_{v}+x_{j} c_{v}\right]\right\}_{v} \\
& =\sum_{v \in S}\left\{\left[z_{v}\right] \cup\left[w_{v}\right]\right\}_{v}+\sum_{v \in S}\left\{\left[z_{v}\right] \cup\left[\ell_{v}-x_{j} \lambda_{v}\right]\right\}_{v} \\
& =\sum_{v \in S}\left\langle s_{v}, t_{v}\right\rangle_{v}+\sum_{v \in S}\left\langle s_{v},\left[\ell_{v}^{\prime}\right]\right\rangle_{v}
\end{aligned}
$$

Here we set $\ell_{v}^{\prime}=\ell_{v}-x_{j} \lambda_{v}$, which is indeed a cocycle as one readily checks:

$$
d \ell_{v}^{\prime}=d \ell_{v}-x_{j} d \lambda_{v}=d \ell_{v}-x_{j}\left(x_{j}^{-1} d \ell_{v}\right)=0 .
$$

We also note that the last equality of (B.11) is just the definition of the local Tate duality: $\left\langle\left[a_{v}\right],\left[b_{v}\right]\right\rangle_{v}=\left\{\left[a_{v}\right] \cup\left[b_{v}\right]\right\}_{v}$.

Completion of the proof. Let us complete the verification of (B.5). Due to the previous computation (B.11), it suffices to show that $\left\langle s_{v},\left[\ell_{v}^{\prime}\right]\right\rangle_{v}$ equals 0 for each place $v$ in $S$. Since $s_{v}$ is an element of $L_{\text {str }}\left(F_{v}, \mathcal{A}_{\eta,(j-1)}\right)\left[x_{j}\right]$ and $L_{\text {str }}^{*}\left(F_{v}, \mathcal{T}_{\eta,(j-1)}^{*}\right)$ is defined as the orthogonal compliment of $L_{\text {str }}\left(F_{v}, \mathcal{A}_{\eta,(j-1)}\right)$ with respect to the local Tate pairing $\langle,\rangle_{v}$, the triviality of $\left\langle s_{v},\left[\ell_{v}^{\prime}\right]\right\rangle_{v}$ is reduced to the following claim:

Claim: For each place $v$ in $S$, the 1-cocycle $\ell_{v}^{\prime}$ represents a cohomology class contained in $\left.\overline{L_{\mathrm{str}}^{*}\left(F_{v}\right.}, \mathcal{T}_{\eta,(j-1)}^{*}\right)+x_{j} H_{\mathrm{cts}}^{1}\left(F_{v}, \mathcal{T}_{\eta,(j-1)}^{*}\right)$

The following lemma is a technical key to the verification of the claim. Let

$$
\delta_{v,(j)}^{1}: H_{\mathrm{cts}}^{1}\left(F_{v}, \mathcal{T}_{\eta,(j)}^{*}\right) \rightarrow H_{\mathrm{cts}}^{2}\left(F_{v}, \mathcal{T}_{\eta,(j-1)}^{*}\right)
$$

denote the connecting homomorphism of the cohomological long exact sequence associated to (3.34).

Lemma B.3. Let $v$ be a finite place in $S$ and let $\bar{\xi}_{v}$ be an arbitrary cocycle of $C^{1}\left(F_{v}, \mathcal{T}_{\eta,(j)}^{*}\right)$ representing a cohomology class contained in $L_{\mathrm{str}}^{*}\left(F_{v}, \mathcal{T}_{\eta,(j)}^{*}\right)$. Further assume that the image of the cohomology class $\left[\bar{\xi}_{v}\right]$ under $\delta_{v,(j)}^{1}$ is trivial when $v$ is a place belonging to $\Sigma_{p}^{c}$. Then there exists a lift $\tilde{\xi}_{v}$ of $\xi_{v}$ to $C^{1}\left(F_{v}, \mathcal{T}_{\eta,(j-1)}^{*}\right)$ such that $\tilde{\xi}_{v}$ is also a cocycle and it represents a cohomology class in $L_{\mathrm{str}}^{*}\left(F_{v}, \mathcal{T}_{\eta,(j-1)}^{*}\right)$. 
Proof of Lemma B.3. The statement is nontrivial only when $v$ is a place in $\Sigma_{p}^{c}$ or a place not lying above $p$ such that the inertia group $I_{v}$ acts trivially on $\mathcal{T}_{\eta,(k)}^{*}$; otherwise the local condition $L_{\mathrm{str}}^{*}\left(F_{v}, \mathcal{T}_{\eta,(k)}^{*}\right)$ is trivial and there is nothing to prove. In the former case, the local condition $L_{\mathrm{str}}^{*}\left(F_{v}, \mathcal{T}_{\eta,(k)}^{*}\right)$ coincides with the whole cohomology group $H_{\mathrm{cts}}^{1}\left(F_{v}, \mathcal{T}_{\eta,(k)}^{*}\right)$. The assumption $\delta_{v,(j)}^{1}\left(\left[\bar{\xi}_{v}\right]\right)=0$ then guarantees the existence of a cohomology class $\left[\tilde{\xi}_{v}^{\prime}\right]$ of $H_{\text {cts }}^{1}\left(F_{v}, \mathcal{T}_{\eta,(j-1)}^{*}\right)$ whose image in $H_{\text {cts }}^{1}\left(F_{v}, \mathcal{T}_{\eta,(j)}^{*}\right)$ coincides with $\left[\bar{\xi}_{v}\right]$. In the latter case, the local condition $L_{\mathrm{str}}^{*}\left(F_{v}, \mathcal{T}_{\eta,(k)}^{*}\right)$ is isomorphic to $H_{\mathrm{cts}}^{1}\left(D_{v} / I_{v}, \mathcal{T}_{\eta,(k)}^{*}\right)$. Consider the cohomological long exact sequence associated to (3.34):

$$
H_{\mathrm{cts}}^{1}\left(D_{v} / I_{v}, \mathcal{T}_{\eta,(j-1)}^{*}\right) \stackrel{(\star)}{\longrightarrow} H_{\mathrm{cts}}^{1}\left(D_{v} / I_{v}, \mathcal{T}_{\eta,(j)}^{*}\right) \longrightarrow H_{\mathrm{cts}}^{2}\left(D_{v} / I_{v}, \mathcal{T}_{\eta,(j-1)}^{*}\right)
$$

Since the procyclic quotient $D_{v} / I_{v} \cong \hat{\mathbb{Z}}$ has cohomological dimension one, the second cohomology group $H_{\mathrm{cts}}^{2}\left(D_{v} / I_{v}, \mathcal{T}_{\eta,(j-1)}^{*}\right)$ should be trivial and hence the natural map $(\star)$ is surjective.

We thus find in both cases a cocycle $\tilde{\xi}_{v}^{\prime}$ of $C^{1}\left(F_{v}, \mathcal{T}_{\eta,(j-1)}^{*}\right)$ representing a cohomology class contained in $L_{\mathrm{str}}^{*}\left(F_{v}, \mathcal{T}_{\eta,(j-1)}^{*}\right)$ such that its image in $C^{1}\left(F_{v}, \mathcal{T}_{\eta,(j)}^{*}\right)$ coincides with $\bar{\xi}_{v}$ up to coboundaries. However, the natural surjection $\mathcal{T}_{\eta,(j-1)}^{*} \rightarrow \mathcal{T}_{\eta,(j)}^{*}$ induces a surjection $C^{\bullet}\left(F_{v}, \mathcal{T}_{\eta,(j-1)}^{*}\right) \rightarrow C^{\bullet}\left(F_{v}, \mathcal{T}_{\eta,(j)}^{*}\right)$ on the cochain complexes, and hence we readily take a lift of an arbitrary coboundary in $C^{1}\left(F_{v}, \mathcal{T}_{\eta,(j)}^{*}\right)$ from the submodule of coboundaries in $C^{1}\left(F_{v}, \mathcal{T}_{\eta,(j-1)}^{*}\right)$. This enables us to construct a desired cocycle $\tilde{\xi}_{v}$ by modifying $\tilde{\xi}_{v}^{\prime}$ in the same cohomology class.

Proof of Proposition B.1. We first recall that the cocycle $\bar{\ell}_{v}$ satisfies $\delta_{v,(j)}^{1}\left(\left[\bar{\ell}_{v}\right]\right)=0$ for every place $v$ in $S$. Indeed, $\delta_{v,(j)}^{1}\left(\left[\bar{\ell}_{v}\right]\right)$ is calculated as $\left[x_{j}^{-1} d \ell_{v}\right]$ due to the definition of the connecting homomorphism $\delta_{v,(j)}^{1}$. Then, the cohomology class $\left[x_{j}^{-1} d \ell_{v}\right]$ is trivial by the equation (B.10) and the fact that $\left[x_{j}^{-1} d \hat{w}\right]=\delta_{2}\left(\left(t_{v}\right)_{v \in S}\right)$ is an element of $\amalg_{\mathcal{T}_{\eta,(j-1)}^{*}}^{2}$. Therefore, by applying Lemma B.3, we find a 1-cocycle $\tilde{\ell}_{v}$ in $C^{1}\left(F_{v}, \mathcal{T}_{\eta,(j-1)}^{*}\right)$ which is a lift of $\bar{\ell}_{v}$ and represents a cohomology class contained in $L_{\mathrm{str}}^{*}\left(F_{v}, \mathcal{T}_{\eta,(j-1)}^{*}\right)$. It is straightforward to see that $\ell_{v}^{\prime}$ is also a lift of $\bar{\ell}_{v}$ by definition, and thus $\tilde{\ell}_{v}-\ell_{v}^{\prime}$ is contained in $\left.x_{j} C^{(} F_{v}, \mathcal{T}_{\eta,(j-1)}^{*}\right)$ due to the exact sequence

$$
0 \rightarrow C^{1}\left(F_{v}, \mathcal{T}_{\eta,(j-1)}^{*}\right) \stackrel{\times x_{j}}{\longrightarrow} C^{1}\left(F_{v}, \mathcal{T}_{\eta,(j-1)}^{*}\right) \rightarrow C^{1}\left(F_{v}, \mathcal{T}_{\eta,(j)}^{*}\right) \rightarrow 0 .
$$

This calculation verifies the claim above, and hence the proof of Proposition B.1 is completed.

\section{REFERENCES}

[AS86] A. Ash and G. Stevens, Modular forms in characteristic $\ell$ and special values of their L-functions, Duke Math. J., 53, no. 3, 849-868 (1986).

[B186] D. Blasius, On the Critical Values of Hecke L-Series, Ann. of Math. (2), 124, no. 1, 23-63 (1986).

[B106] D. Blasius, Hilbert modular forms and the Ramanujan conjecture, in: Noncommutative geometry and number theory, Aspects Math., E37, Vieweg, Wiesbaden, 35-56 (2006).

[BlRo94] D. Blasius and J. D. Rogawski, Motives for Hilbert modular forms, Invent. Math., 114, Issue 1, 55-87 (1994).

[BrLa84] J.-L. Brylinski and J.-P. Labesse, Cohomologie d'intersection et Fonctions L de certaines variétés Shimura, Ann. Sci. Ec. Norm. Super. (4) 17, 361-412 (1984). 
[Ca86] H. Carayol, Sur les représentations p-adiques associées aux formes modulaires de Hilbert, Ann. Sci. Ec. Norm. Super. (4) 19, 409-468 (1986).

[CS05] J. Coates and R. Sujatha, Fine Selmer groups of elliptic curves over p-adic Lie extensions, Math. Ann., 331, 809-839 (2005).

[Da94] A. Dabrowski, p-adic L-functions of Hilbert modular forms, Ann. Inst. Fourier, 44, 1025-1041 (1994).

[De79] P. Deligne, Valeurs de fonctions L et périodes d'intégrales, in: Automorphic forms, representations, and L-functions (Part 2), Proc. Sympos. Pure Math., XXXIII, Providence, R.I., Amer. Math. Soc., 313-346 (1979).

[Di13] M. Dimitrov, Automorphic symbols, p-adic L-functions and ordinary cohomology of Hilbert modular varieties, Amer. J. Math., 135, no. 4 (2013).

[Ge75] S. Gelbart, Automorphic forms on adèle groups, Annals of Mathematics Studies, No. 83. Princeton University Press, 1975.

[Gr76] R. Greenberg, On the Iwasawa invariants of totally real number fields, Amer. J. Math., 98, no. 1, 263-284 (1976).

[Gr78] R. Greenberg, On the structure of certain Galois Groups, Invent. Math., 47, 85-99 (1978).

[Gr94] R. Greenberg, Iwasawa theory and p-adic deformations of motives, in: Motives (Seattle, WA, 1991) Proc. Sympos. Pure Math., 55, 193-223, Part 2, Amer. Math. Soc., Providence, RI (1994).

[Gr06] R. Greenberg, On the structure of certain Galois cohomology groups, Doc. Math. Extra Vol., (4) John H. Coates Sixtieth' Birthday, 335-391 (2006).

[Gr10] R. Greenberg, Surjectivity of the global-to-local map defining a Selmer group, Kyoto J. of Math., Vol. 50, No. 4, 853-888 (2010).

[Gr] R. Greenberg, On the structure of Selmer groups, preprint, available at his webpage: http://www.math.washington.edu/ greenber/research.html

[Ha85] H. Hasse, Über die Klassenzahl abselscher Zahlkörper, Springer-Verlag (1985).

[He82] G. Henniart, Représentations $\ell$-adiques abéliennes, in: Seminaire de theorie des nombres, Seminaire Delange-Pisot-Poitou, Paris 1980-81, Birkhäuser, 107-126 (1982).

[Hi88] H. Hida, On p-adic Hecke algebras for GL(2) over totally real fields, Ann. of Math., 128, 295-384 (1988).

[Hi89] H. Hida, Nearly ordinary Hecke algebras and Galois representations of several variables, in: Algebraic analysis, geometry, and number theory (Baltimore, MD, 1988), 115-134, Johns Hopkins Univ. Press, Baltimore, MD (1989).

[Hi06] H. Hida, Hilbert modular forms and Iwasawa theory, Oxford Mathematical Monographs, Oxford University Press (2006).

[HT93] H. Hida and J. Tilouine, Anticyclotomic Katz p-adic L-functions and congruence modules, Ann. Sci. Éc. Norm. Supér. (4) 26, no. 2, 189-259 (1993).

[HT94] H. Hida and J. Tilouine, On the anticyclotomic main conjecture for CM fields, Invent. Math., 117, no. 1, 89-147 (1994).

[Hsi14] M.-L. Hsieh, Eisenstein congruence on unitary groups and Iwasawa main conjecture for CM fields, J. Amer. Math. Soc., 27, no. 3, 753-862 (2014).

[Kato04] K. Kato, p-adic Hodge theory and values of zeta functions of modular forms, in: Cohomologies p-adiques et applications arithmetiques III, Astérisque 295, 117-290 (2004).

[Katz78] N. M. Katz, p-adic L-functions for CM fields, Invent. Math., 49, 199-297 (1978).

[Ki94] K. Kitagawa, On standard p-adic L-functions of families of elliptic cusp forms, in: p-adic monodromy and the Birch and Swinnerton-Dyer conjecture (Boston, MA, 1991), vol. 165 of Contemp. Math., Amer. Math. Soc., Providence, RI, 81-110 (1994).

[LL79] J.-P. Labesse and R. P. Langlands, L-indistinguishability for SL(2), Canad. J. Math., 31, no. 4, 726-785 (1979).

[Man76] J. I. Manin, Non-Archimedean integration and p-adic Jacquet-Langlands L-functions, Uspehi Mat. Nauk., 31, 5-54 (1976).

[MTT86] B. Mazur, J. Tate and J. Teitelbaum, On p-adic analogues of the conjectures of Birch and Swinnerton-Dyer, Invent. Math., 84, 1-48 (1986).

[MW84] B. Mazur and A. Wiles, Class fields of abelian extensions of $\mathbb{Q}$, Invent. Math., 76 no. 2, 179-330 (1984).

[Mi71] T. Miyake, On automorphic forms on $G L_{2}$ and Hecke operators, Ann. of Math., Second Series, Vol. 94, no. 1, 174-189 (1971). 
[Mok09] C.-P. Mok, The exceptional zero conjecture for Hilbert modular forms, Compos. Math., 145, no. 1, 1-55 (2009).

[NSW00] J. Neukirch, A. Schmidt and K. Wingberg, Cohomology of number fields, Grundlehren der Math., Wissenschaften, 323, Springer (2000).

[Och05] T. Ochiai, Euler system for Galois deformations, Ann. Inst. Fourier (Grenoble) 55, no. 1, 113-146 (2005).

[Och12] T. Ochiai, Several variable p-adic L-functions for Hida families of Hilbert modular forms, Doc. Math., 17, 807-849 (2012).

[OP] T. Ochiai and K. Prasanna, Two-variable Iwasawa theory for Hida families with complex multiplication, in preparation.

[Oh83] M. Ohta, On the zeta function of an abelian scheme over the Shimura curve, Jpn. J. Math., 9, 1-26 (1983).

[Pa94] A. A. Panchishkin, Motives over totally real fields and p-adic L-functions, Ann. Inst. Fourier, 44 989-1023 (1994).

[PR81] B. Perrin-Riou, Groupe de Selmer d'une courbe elliptique à multiplication complexe, Compositio Math., 43, no. 3, 387-417 (1981).

[Ri77] K. A. Ribet, Galois representations attached to eigenforms with nebentypus, in: Modular functions of one variable: V (Proc. Second Internat. Conf., Univ. Bonn, Bonn, 1976), pp. 17-51, Lecture Notes in Math., Vol. 601, Springer, Berlin (1977).

[Ro84] D. Rohrlich, On L-functions of elliptic curves and cyclotomic towers, Invent. Math., 75, 409-423 (1984).

[Ro88] D. Rohrlich, L-functions and division towers, Math. Ann., 281, 611-632 (1988).

[Ru91] K. Rubin, The "main conjectures" of Iwasawa theory for imaginary quadratic fields, Invent. Math., 103, no. 1, 25-68 (1991).

[Ru00] K. Rubin, Euler systems, Annals of Mathematics Studies, vol. 147, Princeton: Princeton University Press (2000).

[Sc86] N. Schappacher, Periods of Hecke characters, Lecture Notes in Mathematics, 1301. SpringerVerlag, Berlin, 1988.

[Se98] J.-P. Serre, Abelian l-adic representations and elliptic curves, Research Notes in Mathematics, 7, A K Peters, Ltd., Wellesley, MA (1998).

[Sh78] G. Shimura, The special values of the zeta functions associated with Hilbert modular forms, Duke Math. J., 45, no. 3, 637-679 (1978).

[Sh94] G. Shimura, Introduction to the arithmetic theory of automorphic functions, Reprint of the 1971 original, Publications of the Mathematical Society of Japan, 11, Princeton University Press, Princeton, NJ (1994).

[St85] G. Stevens, The cuspidal group and special values of L-functions, Trans. Amer. Math. Soc., 291, no. 2,519-550 (1985).

[SU14] C. Skinner and E. Urban, The Main Conjecture for GL(2), Invent. Math., 195, Issue 1, 1-277 (2014).

[Tat63] J. Tate, Duality theorems in Galois cohomology over number fields, Proc. Internat. Congr. Mathematicians (Stockholm, 1962), Inst. Mittag-Leffler, Djursholm, 288-295 (1963).

[Tay89] R. Taylor, On Galois representations associated to Hilbert modular forms, Invent. Math., 98, 265-280 (1989).

[Tay95] R. Taylor, On Galois representations associated to Hilbert modular forms: II, in: Elliptic curves, modular forms and Fermat's last theorem, Ser. Number Theory, I, Int. Press, Cambridge, MA, 185-191 (1995).

[Wa97] L. C. Washington, Introduction to Cyclotomic Fields, 2nd edition, Graduate Texts in Mathematics, 83, Springer-Verlag (1997).

[Wi86] A. Wiles, On p-adic representations for totally real fields, Ann. Math. (2) 123, $407-456$ (1986).

[Wi88] A. Wiles, On ordinary $\lambda$-adic representations associated to modular forms, Invent. Math., 94, 529-573 (1988).

[Win80] J.-P. Wintenberger, Structure galoisienne de limites projectives d'unites locales, Comp. Math., 42, no. 1, 89-103 (1980/1981).

(T. Hara) School of Science and Technology for Future Life, Tokyo Denki University, 5 Senju-Asahi-Cho, Adachi-Ku, Tokyo 120-8551, Japan 
(T. Ochiai) Graduate School of Science, Osaka University, 1-1 Machikaneyama-cho, ToyONAKA, OsAKA 560-0043, JAPAN

E-mail address, T. Hara: t-hara@mail.dendai.ac.jp

E-mail address, T. Ochiai: ochiai@math.sci.osaka-u.ac.jp 